\title{
MBA-DRR: A Delay-Reducing Routing Protocol for Multi-Beam Directional Antennas in Multi-Hop Ad Hoc Networks
}

\author{
by \\ Jean-Daniel Medjo Me Biomo, M.A.Sc. \\ A Thesis submitted to the \\ Department of Systems and Computer Engineering \\ in partial fulfillment of the requirements for the degree of
}

Doctor of Philosophy in Electrical and Computer Engineering

Ottawa-Carleton Institute for Electrical and Computer Engineering

Department of Systems and Computer Engineering

Carleton University

Ottawa, Ontario

May, 2019

(C) Copyright

Jean-Daniel Medjo Me Biomo, 2019 
The undersigned hereby recommends to the

Department of Systems and Computer Engineering acceptance of the Thesis

\title{
MBA-DRR: A Delay-Reducing Routing Protocol for Multi-Beam Directional Antennas in Multi-Hop Ad Hoc Networks
}

\author{
submitted by Jean-Daniel Medjo Me Biomo, M.A.Sc. \\ in partial fulfillment of the requirements for the degree of \\ Doctor of Philosophy in Electrical and Computer Engineering \\ Doctor David Brown, External Examiner \\ Professor Thomas Kunz, Thesis Supervisor \\ Professor Marc St-Hilaire, Thesis Co-supervisor \\ Professor Yvan Labiche, Chair, \\ Department of Systems and Computer Engineering \\ Ottawa-Carleton Institute for Electrical and Computer Engineering \\ Department of Systems and Computer Engineering \\ Carleton University
}

May, 2019 


\section{Abstract}

Ad hoc networks are infrastructure-less and self-organizing networks that consist of static or mobile nodes with limited bandwidth, computing ability and energy. These networks are deployed for a wide range of civilian and military applications. Having an efficient and reliable routing protocol for communication between the nodes can be critical. Our goal in this thesis is to exploit Multi-Beam directional Antennas (MBAs) to significantly reduce the end-to-end (E2E) delay in multi-hop ad hoc networks that service multiple traffic flows. We conduct this work in four major steps. First, we explore the benefits of directional antennas, from the standpoint of single-beam ones, for traditional routing protocols. We propose a single-beam directional antenna MAC protocol in the process. Secondly, using Flying Ad hoc Networks (FANETs) as an example, we make the case that MBAs are yet to be exploited for E2E delay reduction. To that end, we propose a multi-beam directional antenna MAC protocol. Third, as a consequence of the case made in the second step, we propose a Mixed Integer Linear Programming (MILP) model that exploits MBAs' capabilities for delay minimization. Solving this model shows that the routes that are selected for the different flows need to have certain key characteristics that depart from the widespread traditional shortest-route philosophy. Based on these characteristics, we design, in the fourth step, an MBA-Delay-Reducing Routing protocol (MBA-DRR) that fully exploits the benefits of MBAs for delay reduction. The benefits of this protocol apply to all types of multi-hop MBA-based ad hoc networks, both mobile and static. As a matter of fact, the evaluation on a multi-flow static scenario shows that MBA-DRR, with a delay of just $4.4 \mathrm{~ms}$, gets very close to the optimal solution that has a delay of $2.5 \mathrm{~ms}$. Comparatively, Reactive-Geographic hybrid Routing (RGR), a shortest-route-based protocol, has a delay of $48 \mathrm{~ms}$. An evaluation on a representative multi-flow mobile

scenario shows that, while single-beam directional MAC reduces the E2E latency from $700 \mathrm{~ms}$ to $40 \mathrm{~ms}$, and multi-beam directional MAC halves this to $20 \mathrm{~ms}$, our proposed routing protocol further cuts it to $9 \mathrm{~ms}$. 


\section{Table of Contents}

Abstract $\quad$ iii

Table of Contents $\quad$ iv

List of Tables $\quad$ ix

List of Figures $\quad$ xii

$\begin{array}{ll}\text { Acronyms } & \text { Xv }\end{array}$

List of Notations $\quad$ xx

1 Introduction $\quad 1$

1.1 Background . . . . . . . . . . . . . . . . . . 1

1.2 Problem Statement and Research Objective . . . . . . . . . . . 3

1.3 Contributions ....................... 4

1.4 Organization ......................... 6

2 Single-Beam Directional Antennas $\quad 7$

2.1 Introduction . . . . . . . . . . . . . . . . . . 7

2.2 Summary of the Considered Routing Protocols . . . . . . . . . . . . . 8

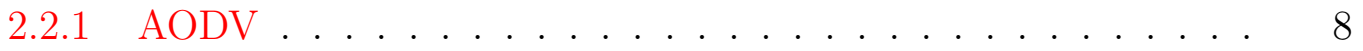

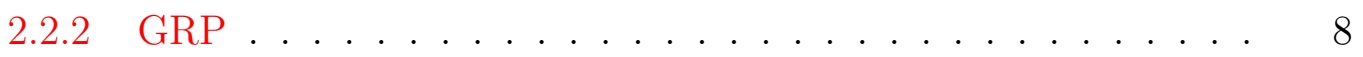

2.2 .3 OLSR ............................... 9

2.2 .4 RGR . . . . . . . . . . . . . . . . . . 10

2.3 Antenna Model . . . . . . . . . . . . . . . . . . . . . . . . 10

2.4 Directional MAC Protocols . . . . . . . . . . . . . . . . . . 13

2.4.1 Key Challenges of MAC Protocols in Radio Networks . . . . . 13 
2.4.2 Proposed Directional MAC Protocol Design . . . . . . . . . 13

2.4.2.1 Broadcasting . . . . . . . . . . . . . . 14

2.4.2.2 Neighbor Table . . . . . . . . . . . . . . . 15

$2.4 .2 .3 \quad \mathrm{RTS} / \mathrm{CTS} \ldots \ldots \ldots \ldots \ldots \ldots$

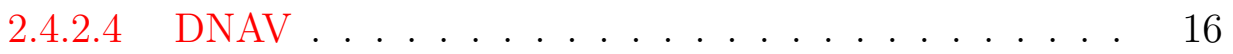

2.4.2.5 Hidden-Terminal Problem . . . . . . . . . . . 16

2.4.2.6 Deafness Problem . . . . . . . . . . . . . 16

2.4.2.7 Exposed-Terminal Problem . . . . . . . . . . 17

2.5 Simulation and Analysis . . . . . . . . . . . . . . . . 17

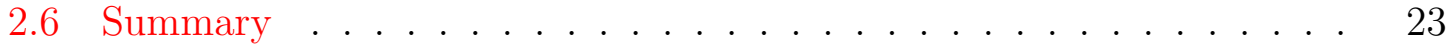

3 Multi-Beam Antennas: Description and Literature Review on Design and Performance $\quad 25$

3.1 Introduction . . . . . . . . . . . . . . . . . 25

3.2 Design of Multi-Beam Antennas . . . . . . . . . . . . . . 26

3.3 Routing Protocols for Multi-Beam Antennas . . . . . . . . . . . . 28

3.4 MAC Protocols for Multi-Beam Antennas . . . . . . . . . . . . . . 29

3.4.1 Centralized Protocols . . . . . . . . . . . . . . . . . . . 29

3.4.2 Distributed Protocols . . . . . . . . . . . . . . . . . . 31

3.5 Performance/Optimization Studies with Multi-Beam Antennas . . . . 37

3.6 Other Performance/Optimization Studies . . . . . . . . . . . 38

3.7 Throughput Maximization vs. Delay Minimization . . . . . . . . . 40

3.8 Summary . . . . . . . . . . . . . . . . . . . . . 43

4 MBA-DbMAC: A Random-Access MAC Protocol for MBAs 47

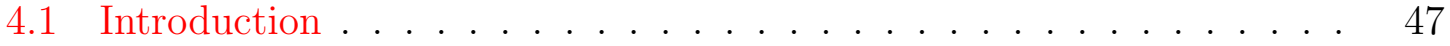

4.2 Bottom Line . . . . . . . . . . . . . . . . . . . . . . . . 48

4.3 MBA Challenges . . . . . . . . . . . . . . . . . . . 49

4.3.1 Synchronization for CPT and CPR . . . . . . . . . 49

4.3 .2 Head-of-Line Blocking . . . . . . . . . . . . . . . . 49

4.3 .3 Deafness . . . . . . . . . . . . . . . . . 5 50

4.3 .4 Hidden-Terminal . . . . . . . . . . . . . . . . . . . 50

4.3.5 Exposed-Terminal . . . . . . . . . . . . . . . . . 51

4.3.6 Random Backoff: Beam-Based or Node-Based . . . . . . . . . 53

4.4 Antenna Model . . . . . . . . . . . . . . . . . . . . . . . 53 
4.5 A Hybrid MAC Solution . . . . . . . . . . . . . . . . 54

4.6 Our Solution: The MBA-DbMAC Protocol . . . . . . . . . . . . 56

4.6 .1 Design . . . . . . . . . . . . . . . . . 57

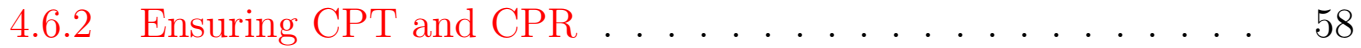

4.6.3 Switch from Tx to Rx mode . . . . . . . . . . . . . 59

4.6.4 Per-Beam Queues . . . . . . . . . . . . . . . . 62

4.6 .5 Broadcasting . . . . . . . . . . . . . . . . 62

4.6 .6 RTS/CTS . . . . . . . . . . . . . . . . . 63

4.6 .7 DNAV . . . . . . . . . . . . . . . . . . 64

4.6 .8 Neighbor Table . . . . . . . . . . . . . . . . . . . . 64

4.7 MBA-DbMAC Functionality Testing . . . . . . . . . . . 65

4.7.1 Uni-Packet Transmission Test . . . . . . . . . . . . . . 65

4.7 .2 Broadcasting Test . . . . . . . . . . . . . . 66

4.7 .3 CPT-CPR Test . . . . . . . . . . . . . . . . . 68

4.7.4 Rx Mode vs Tx Mode . . . . . . . . . . . . . . . . . . 70

4.7.5 Performance Comparison: Delay, Throughput, and Goodput . 72

4.8 Summary . . . . . . . . . . . . . . . . . . . . . 75

5 An Optimization Model for End-to-End Delay Reduction in Ad Hoc $\begin{array}{ll}\text { Networks } & \mathbf{7 8}\end{array}$

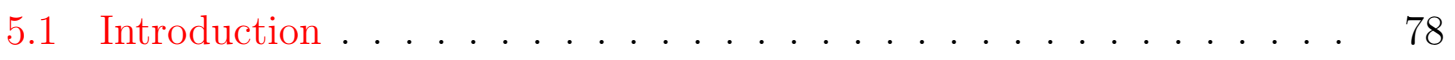

5.2 Scheduling Problem Formulation _ . . . . . . . . . . . 79

5.2 .1 Assumptions . . . . . . . . . . . . . . . . . . . . . . . . 79

5.2 .2 Optimization Model . . . . . . . . . . . . . . . . . . 80

5.3 Model Results and Analysis . . . . . . . . . . . . . . . . . 84

5.3 .1 Topology $1 \ldots \ldots \ldots \ldots \ldots \ldots$

5.3 .2 Topology $2 \ldots \ldots \ldots \ldots$

5.4 Considering Antenna Beams . . . . . . . . . . . . . . . . . 94

5.4.1 Modified Optimization Model . . . . . . . . . . . . . . 94

5.4.2 Modified-Model Results and Analysis . . . . . . . . . . . . 98

5.5 Summary . . . . . . . . . . . . . . . . . . . . . 102

6 The MBA-DRR Routing Protocol 103

6.1 Introduction . . . . . . . . . . . . . . . . . . 103

6.2 A Centralized Approach . . . . . . . . . . . . . . . . . 104 
6.3 A Distributed Heuristic Approach . . . . . . . . . . . . . . . 107

6.4 The Next Step . . . . . . . . . . . . . . . . . . . . . . . . . . . 111

6.5 The Metrics . . . . . . . . . . . . . . . . . . . . . . . . . . . 112

6.6 New Concepts and Changes from RGR . . . . . . . . . . . . . 114

6.7 Message Formats . . . . . . . . . . . . . . . . . . 116

6.8 Protocol Operation . . . . . . . . . . . . . . . . . 117

6.8.1 Maintaining Sequence Numbers . . . . . . . . . . . . . 118

6.8.2 Route Table Entries Update/Creation . . . . . . . . . . . . 118

6.8 .3 RREQs . . . . . . . . . . . . . . . . . . . . . 118

6.8 .4 RREPs . . . . . . . . . . . . . . . . . . 121

6.8 .5 HELLOs . . . . . . . . . . . . . . . . . . . . 123

6.8.6 Route Break, GGF Mode . . . . . . . . . . . . . . 125

6.9 Protocol Operation Example . . . . . . . . . . . . . . . . . 125

6.10 Summary . . . . . . . . . . . . . . . . 135

7 Evaluation of the MBA-DRR Routing Protocol 136

7.1 Introduction . . . . . . . . . . . . . . . . . 136

7.2 Static Scenarios: Comparing Opimal Routes, MBA-DRR, and RGR . 137

7.2.1 Running-Example Static Scenario . . . . . . . . . . . . 137

7.2.1.1 Comparison by Time Slot Scheduling . . . . . . . . 137

7.2.1.2 Comparison in Simulator . . . . . . . . . . . 141

7.2 .2 Random Static Scenarios . . . . . . . . . . . . . . . 142

7.3 Mobile Scenario: Comparing MBA-DRR, RGR, and AODV . . . . 144

7.4 Further Parameterized Evaluation of MBA-DRR . . . . . . . . . . . . 149

7.4.1 Varying Network Size . . . . . . . . . . . . . . . . . . . 151

7.4.2 Varying Traffic Intensity . . . . . . . . . . . . . . . 151

7.4.3 Varying the Beamwidth . . . . . . . . . . . . . . . 154

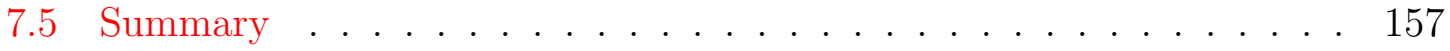

8 Conclusions and Future Work 159

8.1 Conclusions . . . . . . . . . . . . . . . . . . . . . . . . . 159

8.2 Future Work . . . . . . . . . . . . . . . . . . . . . . . . . 161

$\begin{array}{ll}\text { List of References } & 164\end{array}$ 
Appendix A Additional Tables and Figures for Chapter 6

A.1 Routing Tables after Initial Route Discovery of All Flows. . . . . . . 174

A.2 Routing Tables after Delivery of the 1st Data Packet of Each Flow. . 178

Appendix B Additional Tables and Figures for Chapter 7

182 


\section{List of Tables}

2.1 Beamwidth-Dimension Matching . . . . . . . . . . . . 18

2.2 Simulation Parameters . . . . . . . . . . . . . . . . . . . 19

3.1 Minimum Average Delay Link Scheduling . . . . . . . . . . . . . . . . 44

3.2 Delay Within Bounds . . . . . . . . . . . . . . . . . . . . 44

5.1 Notation for MBA-DM-1 . . . . . . . . . . . . . . 81

5.2 Topology 1, Optimal Link Scheduling with 8 Flows . . . . . . . . . 86

5.3 Topology 1, Optimal Link Scheduling with 8 Flows, No MBA . . . . 88

5.4 Topology 2, Optimal Link Scheduling with 6 Flows . . . . . . . . . . 90

5.5 Topology 2, Optimal Link Scheduling with 6 Flows, No MBA . . . . 92

5.6 Topology 2, Link Scheduling with 6 Flows, Shortest Path . . . . . . . 93

5.7 Topology 2, Link Scheduling with 6 Flows, Shortest Path, No MBA . 93

5.8 Notation for MBA-DM-2 . . . . . . . . . . . . . . . . . 95

5.9 Topology 2, Optimal Link Scheduling with $45^{\circ}$ Beamwidth . . . . . . 99

5.10 Topology 2, Optimal Link Scheduling with $60^{\circ}$ Beamwidth . . . . . . 100

5.11 Topology 2, Optimal Link Scheduling with $120^{\circ}$ Beamwidth . . . . . 101

6.1 The SBR/PHC Measure . . . . . . . . . . . . . . . . . 113

6.2 Routing Table Fields in MBA-DRR . . . . . . . . . . . . . . 116

6.3 RREQ Fields . . . . . . . . . . . . . . . . . . . 116

6.4 RREP Fields . . . . . . . . . . . . . . . . . . . . . 116

6.5 HELLO Message Fields Values . . . . . . . . . . . . . . . . . . . . . . 124

6.6 Initial State of the Routing Tables . . . . . . . . . . . . . . . . 127

6.7 Routing Table of Node 6, Step 1 . . . . . . . . . . . . . . . . 128

6.8 RREQ Re-broadcast from Node 3 . . . . . . . . . . . . . . . . 129

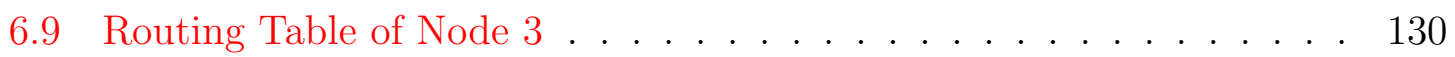

6.10 Routing Table of Node 1 . . . . . . . . . . . . . . . . . 131

6.11 Routing Table of Node 6 . . . . . . . . . . . . . . . . . . . . . 131

6.12 Routing Table of Node 3 After Re-discovery, 1st Route . . . . . . . . 133 


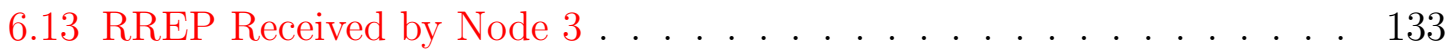

6.14 Routing Table of Node 3 After Re-discovery, 2nd Route . . . . . . . . 134

7.1 Optimal Link Scheduling for Optimal Routes . . . . . . . . . . . . 139

7.2 Optimal Link Scheduling for MBA-DRR Routes . . . . . . . . . . 139

7.3 Optimal Link Scheduling for RGR Routes . . . . . . . . . . . 140

7.4 Path Length and Delay for Optimal Link Scheduling . . . . . . . . 143

7.5 Path Length and Number of Queuings for MBA-DRR . . . . . . . . 143

7.6 Path Length and Number of Queuings for RGR . . . . . . . . . 143

7.7 Network Size Dimensioning _. . . . . . . . . . . . . . . . 151

7.8 Traffic Intensity Dimensioning in a 10-Node Network . . . . . . . . 153

7.9 Varying Beamwidth in a 10-Node Network . . . . . . . . . . . . 154

A.1 Routing Table of Node 1 . . . . . . . . . . . . . . . . . . 174

A.2 Routing Table of Node $2 \ldots \ldots \ldots \ldots$

A.3 Routing Table of Node $3 \ldots \ldots \ldots \ldots$

A.4 Routing Table of Node $4 \ldots \ldots \ldots \ldots \ldots$

A.5 Routing Table of Node $6 \ldots \ldots \ldots \ldots$

A.6 Routing Table of Node $8 \ldots \ldots \ldots$. . . . . . . . . . 176

A.7 Routing Table of Node $10 \ldots \ldots \ldots$

A.8 Routing Table of Node $12 \ldots \ldots \ldots$

A.9 Routing Table of Node $13 \ldots \ldots \ldots$

A.10 Routing Table of Node $14 \ldots \ldots \ldots$

A.11 Routing Table of Node 15 . . . . . . . . . . . . . . . 177

A.12 Routing Table of Node $16 \ldots \ldots \ldots$

A.13 Routing Table of Node $2 \ldots \ldots \ldots$

A.14 Routing Table of Node $4 \ldots \ldots$. . . . . . . . . . . . 178

A.15 Routing Table of Node 8 . . . . . . . . . . . . . . . . . . . . 179

A.16 Routing Table of Node 10 . . . . . . . . . . . . . . . . 179

A.17 Routing Table of Node 12 . . . . . . . . . . . . . . . 179

A.18 Routing Table of Node $13 \ldots \ldots$

A.19 Routing Table of Node $14 \ldots \ldots$. . . . . . . . . 180

A.20 Routing Table of Node $15 \ldots \ldots \ldots$

A.21 Routing Table of Node 16 . . . . . . . . . . . . . . . . . . 181

B.1 Optimal Link Scheduling for 10-Node Scenario 1 . . . . . . . . . 183

B.2 Optimal Link Scheduling for 10-Node Scenario $2 \ldots \ldots \ldots \ldots$ 
B.3 Optimal Link Scheduling for 10-Node Scenario 3 . . . . . . . . . . . . 185

B.4 Optimal Link Scheduling for 10-Node Scenario 4 . . . . . . . . . . . 186

B.5 Optimal Link Scheduling for 10-Node Scenario 5 . . . . . . . . . . . . 187

B.6 Optimal Link Scheduling for 10-Node Scenario 6 . . . . . . . . . 188

B.7 Optimal Link Scheduling for 10-Node Scenario 7 . . . . . . . . . . . . 189

B.8 Optimal Link Scheduling for 10-Node Scenario 8 . . . . . . . . . . . . 190

B.9 Optimal Link Scheduling for 10-Node Scenario 9 . . . . . . . . . . . . 191

B.10 Optimal Link Scheduling for 10-Node Scenario 10 . . . . . . . . . . . 192 


\section{List of Figures}

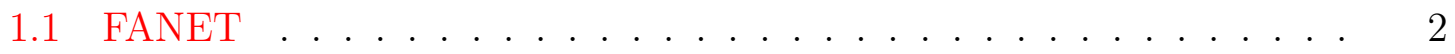

2.1 Antenna Beams Configuration . . . . . . . . . . . . . . . 12

2.2 Hidden-Terminal and Deafness . . . . . . . . . . . . . . . . 14

2.3 Simplified DbMAC Flowchart . . . . . . . . . . . . . 15

2.4 Random Waypoint Trajectory Example . . . . . . . . . . . . . 20

2.5 Routing Performance . . . . . . . . . . . . . . . . . . . 21

3.1 Multi-Beam Antenna Modes [1] @2016 IEEE . . . . . . . . . . . 27

3.2 Concept of ripple-diamond-chain routing [1] @ 2016 IEEE . . . . . . . 28

3.3 Delay Minimization vs. Throughput Maximization . . . . . . . . . . 41

3.4 Minimum Average Delay vs. Higher but Bounded Delays . . . . . . . 44

4.1 CPR and CPT [2] (C)2008 IEEE . . . . . . . . . . . . . . 50

4.2 Hidden-Terminal Problem [3] (C)2012 IEEE . . . . . . . . . . . . . . 51

4.3 Exposed-Terminal Problem [4] . . . . . . . . . . . . . . . . . . . . 52

4.4 Exposed-Terminal Problem with Directional Antennas . . . . . . . 53

4.5 Illustration of Deafness as per [2] (C)2008 IEEE . . . . . . . . . . 55

4.6 Two-tier MAC Approach Vs Traditional Single-tier MAC Approach . 57

4.7 Controller/Parent Diagram . . . . . . . . . . . . . . . . . . 61

4.8 Beam/Child Diagram . . . . . . . . . . . . . . . . . . 61

4.9 MAC Test Scenario Topology _ . . . . . . . . . . . . . . 66

4.10 UPT Test . . . . . . . . . . . . . . . . . . . 67

4.11 Broadcast Test . . . . . . . . . . . . . . . . . 68

4.12 CPT Test . . . . . . . . . . . . . . . . . . . . 69

4.13 CPR Test . . . . . . . . . . . . . . . . . . 71

4.14 MAC Delay, with 5 pkt/sec Traffic Generation Rate . . . . . . . . 73

4.15 Throughput, with 5 pkt/sec Traffic Generation Rate. . . . . . . . . 74

4.16 Throughput, with 105 pkt/sec Traffic Generation Rate . . . . . . 75

4.17 Goodput, with 105 pkt/sec Traffic Generation Rate . . . . . . . . 76 


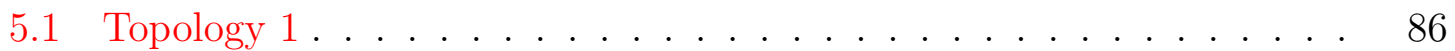

5.2 A Star Node . . . . . . . . . . . . . . . . . . . . . 87

5.3 Topology $2 \ldots \ldots \ldots \ldots$

6.1 Standard SDN Architecture in VANETs [5] (C2016 IEEE . . . . . . . 105

6.2 Topology Change During Optimization . . . . . . . . . . . . 105

6.3 Route in Reactive Mode . . . . . . . . . . . . . . . . . . . . . . 109

6.4 Route in Greedy Geographic Mode . . . . . . . . . . . . . . . . 111

6.5 Flowchart for Processing RREQ . . . . . . . . . . . . . . . . . 122

6.6 Flowchart for Processing RREP . . . . . . . . . . . . . . . . . . . 124

6.7 Static Scenario . . . . . . . . . . . . . . . . . . . 126

6.8 6-Beam Configuration of the Nodes . . . . . . . . . . . . 127

7.1 Static Scenario . . . . . . . . . . . . . . . . . . . . 138

7.2 Delay: Optimal vs MBA-DRR vs RGR . . . . . . . . . . . . . 142

7.3 Delay, 30-Node Mobile Scenario . . . . . . . . . . . . . . 146

7.4 Delay, 30-Node Mobile Scenario, Without Omni … . . . . . 146

7.5 PDR, 30-Node Mobile Scenario . . . . . . . . . . . . . . . . 148

7.6 Control Overhead, 30-Node Mobile Scenario . . . . . . . . . . . 148

7.7 Delay, 30-Node Mobile Scenario, Comparison with AODV . . . . . . 150

7.8 PDR, 30-Node Mobile Scenario, Comparison with AODV . . . . . . 150

7.9 Delay, Mobile Scenario, Varying Network Size . . . . . . . . . . . 152

7.10 PDR, Mobile Scenario, Varying Network Size . . . . . . . . . . . 152

7.11 Delay, 10-Node Mobile Scenario, Varying Traffic Intensity . . . . . . 153

7.12 PDR, 10-Node Mobile Scenario, Varying Traffic Intensity . . . . . . 154

7.13 Delay, 10-Node Mobile Scenario, Varying Beamwidth . . . . . . . 155

7.14 PDR, 10-Node Mobile Scenario, Varying Beamwidth . . . . . . . 156

7.15 Delay, 25-Node Mobile Scenario, Varying Beamwidth . . . . . . . 156

7.16 PDR, 25-Node Mobile Scenario, Varying Beamwidth . . . . . . . 157

B.1 Random 10-Node 5-Flow Static Scenario 1 . . . . . . . . . . 183

B.2 Random 10-Node 5-Flow Static Scenario $2 \ldots \ldots \ldots$

B.3 Random 10-Node 5-Flow Static Scenario 3 . . . . . . . . . . 185

B.4 Random 10-Node 5-Flow Static Scenario 4 . . . . . . . . . . . 186

B.5 Random 10-Node 5-Flow Static Scenario 5 . . . . . . . . . . 187

B.6 Random 10-Node 5-Flow Static Scenario 6 . . . . . . . . . . . . 188

B.7 Random 10-Node 5-Flow Static Scenario 7 . . . . . . . . . . . . 189 
B.8 Random 10-Node 5-Flow Static Scenario 8 . . . . . . . . . . . 190

B.9 Random 10-Node 5-Flow Static Scenario 9 . . . . . . . . . . . . . 191

B.10 Random 10-Node 5-Flow Static Scenario 10 . . . . . . . . . . . 192 


\section{Acronyms}

$\begin{array}{ll}\text { ACG } & \text { Additional Control Gap } \\ \text { AoA } & \text { Angle-of-Arrival } \\ \text { AODV } & \text { Ad-hoc On-demand Distance Vector } \\ \text { AP } & \text { Access Point } \\ \text { BMN } & \text { Best-Moving Node } \\ \text { BS } & \text { Base Station } \\ \text { B2NH } & \text { Beam toNext Hop } \\ \text { B2PH } & \text { Beam to Previous Hop } \\ \text { CCR } & \text { Critical Chain Reception } \\ \text { CCT } & \text { Critical Chain Transmission } \\ \text { CPT/CPR } & \text { Concurrent-Packet-Transmission/Concurrent-Packet-Reception } \\ \text { CSMA/CA } & \text { Carrier-Sense Multiple Access with Collision Avoidance } \\ \text { CTE } & \text { Cooperative Transmission Epoch } \\ \text { CTS } & \text { Clear To Send } \\ \text { CW } & \text { Contention Window } \\ \text { DbMAC } & \text { IEEE 802.11b-based Directional MAC protocol for SBAs } \\ \text { DCF } & \text { Distributed Coordination Function } \\ \text { DIFS } & \text { Distributed Inter-Frame Space } \\ \text { DNAV } & \text { Directional Network Allocation Vector }\end{array}$




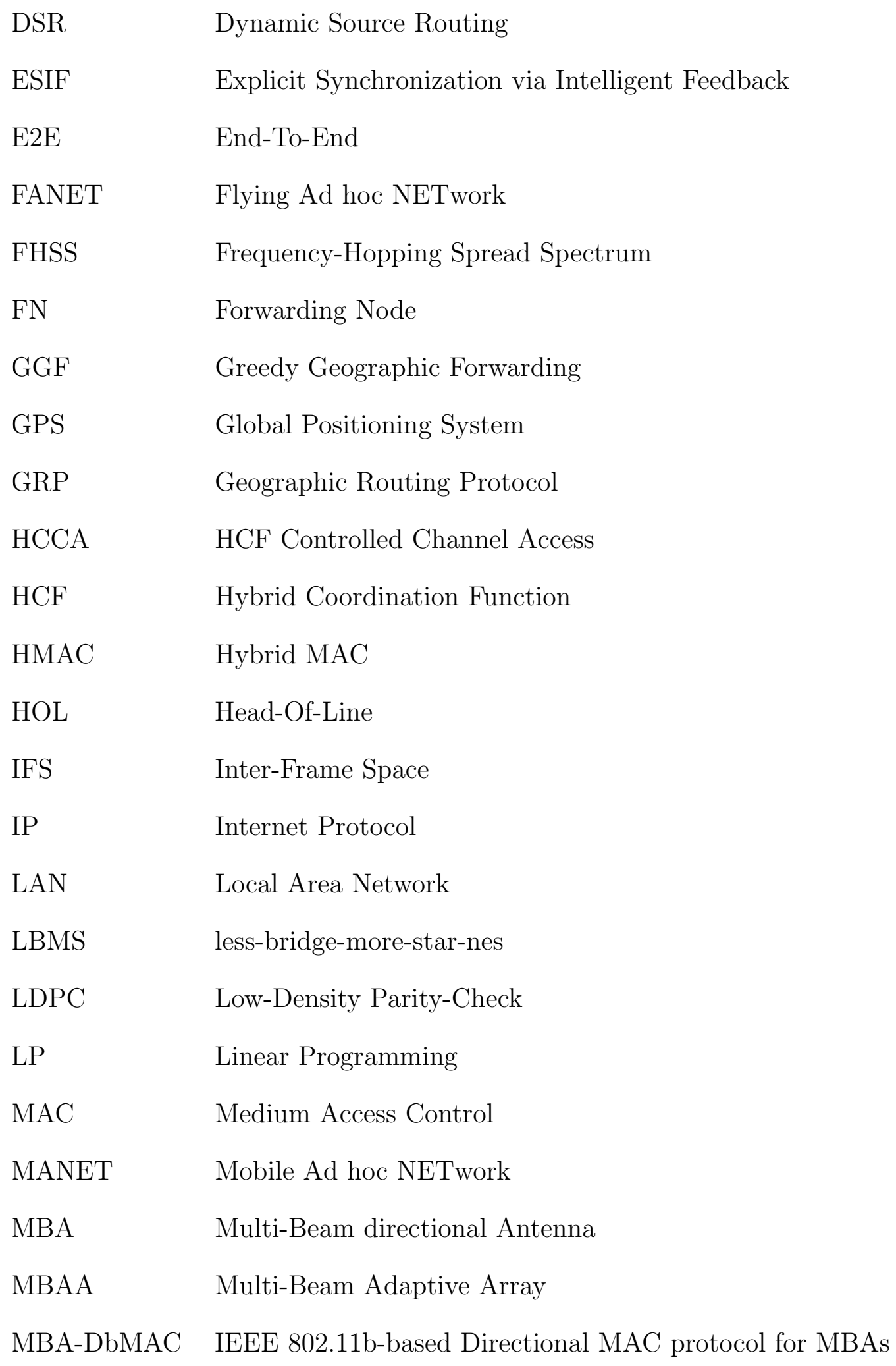




\begin{tabular}{|c|c|}
\hline MBA-DM-1 & Delay Minimization with MBAs, Version 1 \\
\hline MBA-DM-2 & Delay Minimization with MBAs, Version 2 \\
\hline MBA-DRR & Delay Reducing Routing protocol for MBAs \\
\hline MBA-MANET & MANET where all nodes are equipped with MBAs \\
\hline MBDMAC & MAC Protocol for Multi-Beam Directional Antenna \\
\hline MB-URAM & Multi-Beam Uncoordinated Random Access MAC \\
\hline MCSA & Multi-Channel Smart Antenna \\
\hline MFBA & Multiple Fixed-Beam directional Antenna \\
\hline M-HCCA & Multi-beam AP-assisted HCCA \\
\hline MILP & Mixed Integer Linear Programming \\
\hline MIMO & Multiple-Input and Multiple-Output \\
\hline MMAC-NB & Multiple-beam omnidirectional MAC with Node-based Backoff \\
\hline MPC & Multi-Packet Communication \\
\hline MPR & Multi-point Relay \\
\hline MPT/MPR & Multi-Packet-Transmission/Multi-Packet-Reception \\
\hline MR & Multiple Radio \\
\hline MST & Minimum Spanning Tree \\
\hline NAV & Network Allocation Vector \\
\hline NDTDD & Dynamic TDMA-based MAC protocol for Directional antennas \\
\hline NTG & NodeThroughput Gain \\
\hline OLSR & Optimized Link State Routing \\
\hline Omni-MANET & MANET where all nodes are equipped with Omnidirectional antennas \\
\hline OPL & Optimization Programming Language \\
\hline PDR & Packet Delivery Ratio \\
\hline
\end{tabular}




$\begin{array}{ll}\text { PHC } & \text { Practical Hop Count } \\ \text { QoS } & \text { Quality of Service } \\ \text { RDC } & \text { Ripple-Diamond-Chain } \\ \text { RERR } & \text { Route Error } \\ \text { RGR } & \text { Reactive-Geographic hybrid Routing protocol } \\ \text { ROMA } & \text { Receiver-Oriented Multiple Access } \\ \text { RREP } & \text { Route Reply } \\ \text { RREQ } & \text { Route Request } \\ \text { RTS } & \text { Request To Send } \\ \text { SAMPC } & \text { SIR Access MPC } \\ \text { SBA } & \text { Single-Beam directional Antenna } \\ \text { SBA-FANET } & \text { FANET where all nodes are equipped with SBAs } \\ \text { SBA-MANET } & \text { MANET where all nodes are equipped with SBAs } \\ \text { SBR } & \text { Star to Bridge Ratio } \\ \text { SDN } & \text { Software-Defined Network } \\ \text { SINR } & \text { Signal-to-Interference-plus-Noise Ratio } \\ \text { SIR } & \text { Signal-to-Interference Ratio } \\ \text { SSP } & \text { Sector Selection Problem } \\ \text { TAMPC } & \text { Threshold Access MPC } \\ \text { TC } & \text { Topology Control } \\ \text { TDMA } & \text { Time-Division Multiple Access } \\ \text { TTL } & \text { Time To Live } \\ \text { UAV } & \text { Unmanned Aerial Vehicles } \\ \text { UPT } & \text { Uni-Packet Transmission } \\ & \end{array}$




$\begin{array}{ll}\text { VANET } & \text { Vehicular Ad hoc NETwork } \\ \text { WDN } & \text { Wireless Distributed Network } \\ \text { WLAN } & \text { Wireless LAN } \\ \text { WMN } & \text { Wireless Mesh Network } \\ \text { WRMN } & \text { Wireless Relay Mesh Network }\end{array}$




\section{List of Notations}

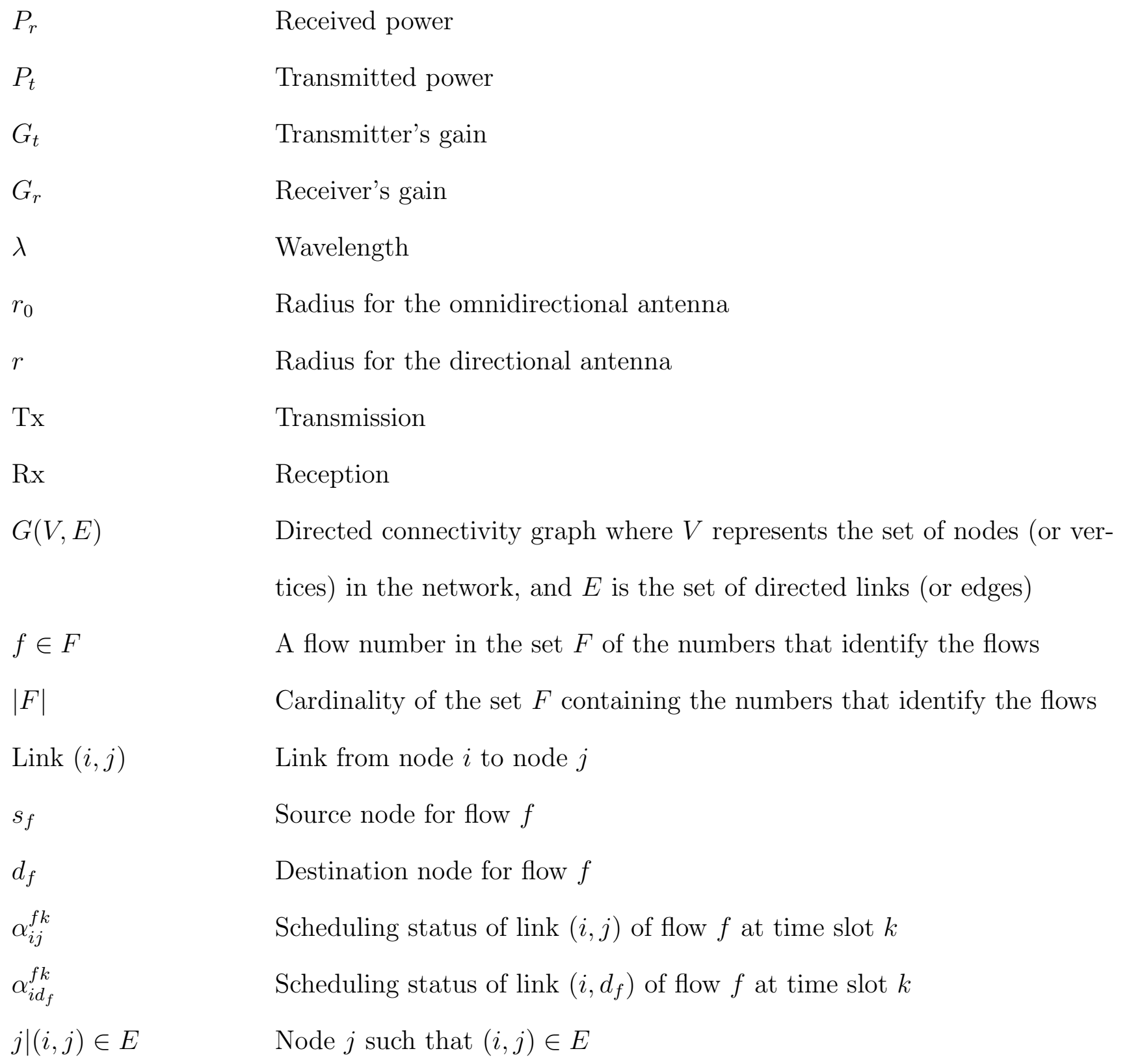


$j \in V \mid(i, j) \in E \quad$ Node $j \in V$ such that $(i, j) \in E$

$\alpha_{i j m}^{f k} \quad$ Scheduling status of link $(i, j)$ of flow $f$, on beam $m$ of node $i$ at time slot $k$

$\alpha_{i d_{f} m}^{f k} \quad$ Scheduling status of link $\left(i, d_{f}\right)$ of flow $f$, , on beam $m$ of node $i$ at time slot $k$

$m \leq M \mid(i, j, m) \in E \quad$ Beam $m$ on which node $i$ reaches node $j . M$ is the number of beams. $\left(X^{(m)}, Y\right) \quad$ Link between nodes $X$ and $Y$, where node $X$ uses its beam number $m$ to reach node $Y$. 


\section{Chapter 1}

\section{Introduction}

\subsection{Background}

Mobile Ad hoc NETworks (MANETs) are wireless networks that consist of mobile nodes with limited bandwidth, computing ability and energy, which is different from traditional wired networks. These nodes are willing to forward packets from other nodes, making the network typically a multi-hop one. MANETs do not have any fixed infrastructure, as opposed to traditional wireless mobile networks such as cellular networks that are supported by a wired fixed infrastructure. Basically, MANETs are self-configuring and self-organizing wireless networks used when there is no possibility to set up a network infrastructure. Their applications include home networking, military and emergency networks.

Flying Ad hoc NETworks (FANETs) [6] are a type of MANETs formed by small and medium-sized Unmanned Aerial Vehicles (UAVs), also referred to as drones. These networks are therefore exclusively airborne. Figure 1.1 depicts the general idea of a FANET with a single traffic flow (a single source-destination pair) whose path is represented with red arrows. In this work, FANETs are used as an example of MANETs when evaluating the protocols.

To date, most MANETs rely on omnidirectional antennas. The problem with this type of antennas is the unnecessary radiation of electromagnetic waves in all directions. This results in large amount of energy being wasted and limited network performance. To overcome this limited network performance, the last decade has 


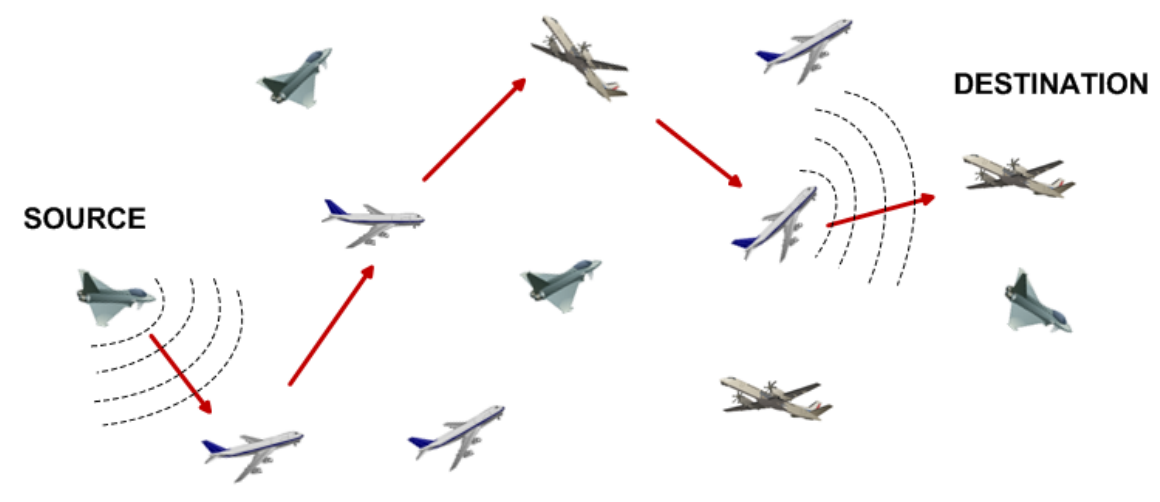

Figure 1.1: FANET

seen a growing interest in trading omnidirectional antennas for directional antennas in MANETs. This interest has been driven by the many advantages that directional antennas have over their omnidirectional counterparts.

In effect, a (single-beam) directional antenna is characterized by a relatively narrow beam pointed in the desired direction. Directional antennas limit the area of radiation, increase spatial reuse, and have the potential to save the battery energy of mobile devices. With the directional gain, the directional antenna substantially increases the transmission range, resulting in an increased coverage area of the network.

Today, although not overly widespread, most MANET applications utilize commercial off-the-shelf WiFi (IEEE 802.11 standard) equipment. WiFi was developed assuming that the transceivers are equipped with omnidirectional antennas. When used for MANETs, many problems arise such as lower network capacity, longer End-To-End (E2E) delays, limited transmission ranges, etc. Hence, alternative techniques have to be designed or re-invented specifically for MANETs. As already noted, directional antennas have several advantages over omnidirectional ones, hence positioning the formers as a promising avenue.

However, the deployment of (single-beam) directional antennas may result in new problems. For example, the deafness problem appears when a node is tuned to a specific direction and thus cannot "hear" a node transmitting from another direction, even if both nodes are within transmission range of each other. The deafness problem impedes dynamic resource allocation and increases the possibility 
of link breakage. To mitigate the deafness problem and enhance the network capacity, Multi-Beam directional Antennas (MBAs) [7] have been proposed. They allow concurrent communications with multiple neighboring nodes while inheriting the many advantages of single-beam directional antennas.

For easy read, we adopt a convention here to indicate the antenna types. In effect, directional antennas can be classified into two groups: Single-Beam directional Antennas (SBAs), and Multi-Beam directional Antennas. From now onward in these pages, the expression "directional antennas" by default means single-beam directional antennas. If the directional antennas are multi-beam, we will specifically call them multi-beam directional antennas or MBAs. For example, a MANET where the nodes are equipped with single-beam directional antennas will be called an SBA-MANET. Likewise, a MANET where the nodes are equipped with multi-beam directional antennas will be called an MBA-MANET. If the nodes are equipped with omnidirectional antennas, then we have an Omni-MANET. The convention is that the hyphened name indicates the type of the antennas in the first part, and the type of network in the second part.

\subsection{Problem Statement and Research Objective}

The goal of our work is to design a routing protocol that will minimize the E2E delay for delay-sensitive applications in multi-flow ad hoc networks. We established in previous work [8] that the Reactive-Geographic hybrid Routing (RGR) protocol is the best protocol for flying ad hoc networks. However, only omnidirectional antennas were used, resulting in a Packet Delivery Ratio (PDR) of about 93\% and an E2E delay of about $40 \mathrm{~ms}$ in a region of $2000 \mathrm{~m} \times 4000 \mathrm{~m}$. Moreover, only a single flow was considered. Despite having 30 mobile nodes that moved randomly, the covered region had to be relatively small because of the limited transmission range $(1000 \mathrm{~m})$ of omnidirectional antennas. From the afore-mentioned previous work, a natural step forward is to improve our routing solution so that it reduces the E2E delay (and/or increases the PDR) in the presence of multiple flows.

Unlike single-flow scenarios/solutions, considering multiple flows is more realistic. 
For instance, real missions that involve UAVs will most likely feature many concomitant conversations between multiple pairs of those UAVs. Furthermore, most real missions are also expected to be very delay-sensitive as they typically involve live video streaming, real-time communication, etc. Therefore, minimizing the E2E delay should justifiably be a major focus for protocol design in ad hoc networks. We make minimizing the E2E delay in multi-flow ad hoc networks the primary focus in this work, without sacrificing the PDR.

Following the above reasoning, we are interested in designing, implementing, and testing a fundamentally better routing protocol that will use directional antennas. Introducing directional antennas has the potential to extend the coverage area. However, we still need to find a way of using them for delay reduction in the context of multiple flows. This is where multi-beam directional antennas come in. Not only can they extend the coverage area, but also they can handle simultaneous servicing of multiple flows. Such concurrent transmissions and receptions have to be smartly exploited by the routing protocol in order to minimize/reduce the E2E delay.

To limit the complexity of the computations and implementations, we are going to only consider two-dimensional (2D) networks in this work. An extension to 3D networks will be subject for future work. However, the design and the core mechanisms of the protocols should not change whether it is $2 \mathrm{D}$ or $3 \mathrm{D}$ that is considered. As pointed out by [9], most of the proposed protocols for ad hoc networks in the literature assume 2D networks where all nodes are distributed in a 2D plane. The extension to 3D brings several challenges that have not been adequately addressed yet. One such challenge has to do with the position information of the nodes. As already mentioned, this extension is beyond the scope of this work.

\subsection{Contributions}

Our ultimate contribution is a novel routing protocol for ad hoc networks. The novelty of our protocol lies in the fact that we fully exploit the benefits of MBAs in order to considerably reduce the E2E delay in multi-flow scenarios. To the best of our knowledge, no attempt has been made thus far to reduce the E2E delay of multi-flow ad hoc networks by capitalizing on the capabilities of MBAs. 
Along the way, we propose a delay-specific optimization model that presents a few advantages. Not only does it allow to target the minimization of the average E2E delay, but also it provides the ability to pursue other E2E-delay-related objectives such as delay-bounding whereby the delay of each individual flow is kept within certain bounds. Therefore, this model can be used to design different delay-aware protocols with different delay-related objectives. Moreover, this model provides an optimal value to compare our protocol with. Based on this contribution, the following papers have been published:

- [10] J.-D. Medjo Me Biomo, T. Kunz, and M. St-Hilaire, "Exploiting multiple beam antennas for end-to-end delay reduction in ad hoc networks," in 9th EAI International Conference on Ad Hoc Networks (AdHocNets 2017), September 2017. Best Paper Award.

- [11] J.-D. Medjo Me Biomo, T. Kunz, and M. St-Hilaire, "Exploiting multibeam antennas for end-to-end delay reduction in ad hoc networks," Mobile Networks and Applications, vol. 23, no. 5, pp. 1293-1305, Oct 2018.

We also propose two MAC protocols for directional antennas: one for singlebeam directional antennas, and one for multi-beam directional antennas. In effect, to explore directional antennas (both single-beam and multi-beam) we need suitable MAC protocols. Not having them readily available, we design our own protocols that introduce novel mechanisms as well. It is worth emphasizing that there are numerous directional (both single-beam and multi-beam) MAC protocols in the literature. What is missing is the implementation of directional MAC protocols for use in our simulator. To date, we have published the work on one of our proposed MAC protocols, the one for SBAs, in the following paper:

- [12] J.-D. Medjo Me Biomo, T. Kunz, and M. St-Hilaire, "Directional antennas in FANETs: A performance analysis of routing protocols," in 2017 InternationalConference on Selected Topics in Mobile and Wireless Networking (MoWNeT),May 2017, pp. 18.

In summary, though the ultimate goal is a delay-reducing routing protocol, the contribution of this work is fourfold with the following outputs: a MAC protocol for single-beam antennas, a MAC protocol for multi-beam antennas, a delay-specific 
optimization model, and a delay-reducing routing protocol for multi-flow ad hoc networks.

\subsection{Organization}

The remainder of this thesis is organized as follows. We first confirm the benefits of SBAs to reduce E2E delay in ad hoc networks such as FANETs in Chapter 2; hence suggesting the potential for even higher benefits with MBAs. In Chapter 3, the potential of MBAs is presented, and the relevant work in the literature that involves MBAs is reviewed. It is shown that MBAs have been widely utilized in wireless networks mostly to improve capacity and throughput, and that little work has been done to exploit the concurrency feature of MBAs to minimize the E2E delay. Our MAC Protocol for MBAs is presented in Chapter 4. The goal of this MAC protocol is to support the most significant MBA capabilities/features. A delayminimization model that harnesses the benefits of MBAs is proposed in Chapter 5. The case is made that MBAs can be exploited to significantly reduce the E2E delay in static ad hoc networks. A formal optimization model for delay reduction is defined, and we learn some key routing-related lessons by solving it. The concepts of star nodes and bridges are introduced. The new routing protocol that we are proposing is presented in Chapter 6. The case against a centralized approach is made, and a distributed heuristic approach is adopted. The details of the design and operation of the protocol are presented. New routing metrics that depart from the typical shortestroute philosophy are defined to select the routes. The concepts of star nodes and bridges are used. The evaluation of the routing protocol is conducted in Chapter 7 . With various scenarios (some static, but most involving mobility), it is shown that the protocol achieves the goal of reducing the E2E delay in multi-flow ad hoc networks. Finally, Chapter 8 concludes the thesis and lays out some avenues for future work. 


\section{Chapter 2}

\section{Single-Beam Directional Antennas}

\section{$2.1 \quad$ Introduction}

A (single-beam) directional antenna is characterized by a relatively narrow beam that can be pointed exclusively in the desired direction [13]. In order to best utilize directional antennas, a suitable MAC protocol must be used. Widely used MAC protocols such as the IEEE 802.11 standard suite are tailored for omnidirectional antennas. Therefore, they do not perform well when directional antennas are used. Many MAC protocols specifically designed for directional antennas in ad hoc networks have been proposed in the literature. With no directional MAC protocol at our disposal (available in our simulator [14]), we need to design and implement a generic directional MAC protocol that is representative of inexpensive (in terms of bandwidth and radio items required) features and mechanisms found in the literature.

In this chapter, we investigate the benefits of using single-beam directional antennas in an example of MANET. In previous work $[8,15,16]$, we proposed a new routing protocol, the Reactive-Geographic hybrid Routing (RGR) protocol for MANETs which assumes omnidirectional antennas. Now, what about routing in $S B A-M A N E T s$ ? To answer this question, we first design and implement a generic directional MAC protocol, and then evaluate the performance of a few representative routing protocols in SBA-MANETs. This evaluation and its analysis make a cogent case for the potential of using single-beam directional antennas in MANETs. For diversity, four very distinct routing protocols are considered: the Ad hoc On-demand Distance Vector (AODV) [17]; the Geographic Routing Protocol (GRP) [18]; the Optimized Link State Routing (OLSR) [19]; and the Reactive-Geographic hybrid 
Routing (RGR) $[8,15,16,20]$.

The remainder of this chapter is organized as follows. Section 2.2 summarizes the considered routing protocols. The directional antenna model is presented in Section 2.3. In Section 2.4, key challenges of MAC protocols, and a summary of our own design of a generic directional MAC protocol are presented. Simulation results of routing in SBA-MANETs are discussed in Section 2.5, and concluding remarks on this chapter are made in Section 2.6.

\subsection{Summary of the Considered Routing Proto- cols}

\subsubsection{AODV}

The Ad-hoc On-demand Distance Vector protocol [17] is a reactive routing protocol. When a source node wants to start a data packet transmission to a destination node to whom it has no valid route, it first initiates a route discovery by broadcasting a Route Request message (RREQ) to its neighbors, and those neighbors, in turn, forward (re-broadcast) the RREQ to their neighbors, etc. When an RREQ reaches the destination node or when an intermediate node happens to have a route to the destination, a Route Reply message (RREP) is generated and sent to the source node. Once a route is established, the data packet transmission starts between the source node and the destination node. However, when the data packet is being routed, it can happen that a next hop node is unreachable (broken link) due to the mobility of the nodes. The intermediate node where this occurs sends out a Route Error (RERR) message to the source node and drops the data packet if local repair is not enabled. When local repair is enabled, the intermediate node holds on to the data packet while it tries to repair the route locally by broadcasting new RREQs in order to establish a new route to the destination.

\subsubsection{GRP}

The Geographic Routing Protocol [18] is a position-based routing protocol built on two assumptions: (1) each node is aware of its own geographic location and the 
location of its immediate neighbors; and (2) the source node is aware of the position of the destination node. Immediate neighbors' locations are updated periodically by means of HELLO messages. Data packets are routed through the network using the geographic location of the destination. GRP operates without routing tables, and routing to the destination relies on the information each node has about its neighbors. A node broadcasts its new position either when it moves more than a user-specified distance (distance limit), or when it crosses a quadrant boundary. Quadrants are square regions whose size is also specified by the user. An entire simulation area is divided in many quadrants. The most commonly-used geographic routing algorithms are greedy forwarding and face routing. In greedy forwarding, the data packet is brought closer to the destination in each step by the holding node forwarding it to the neighbor that reduces the distance to the destination. Greedy forwarding fails if there is no next hop among the neighbors that is closer to the destination. When this happens, greedy forwarding switches over to perimeter mode, where the next hop is selected to traverse the perimeter of the region where greedy forwarding fails. Perimeter mode forwarding continues as long as there is no better greedy next-hop neighbor. In face routing, the regions are considered to be separated by the edges of a planar graph. The algorithm routes the packet along the face; it returns to the point closest to the destination and explores the next face closer to the destination. Face routing always finds a path to the destination. In the GRP version that we use, only greedy forwarding is implemented.

\subsubsection{OLSR}

The Optimized Link State Routing protocol [19] is a table-driven proactive protocol. Routes are continuously stored and updated in tables. Therefore, whenever a route is needed, the protocol presents the route immediately without any initial delay. To reduce the overhead of packet transmission, candidate nodes, called Multipoint Relays (MPRs), are selected and responsible for forwarding broadcast packets during the flooding process. OLSR performs hop-by-hop routing, where each node uses its most recent routing information to route packets. The MPR selection is done in such a way that it covers all of the nodes that are two hops away. A node senses and selects its MPRs with HELLO messages. HELLO messages are sent at a regular time interval. MPR selection is signaled through the HELLO messages. The Topology Control (TC) messages broadcast a subset of the topology information to enable each 
node to build a (partial) network topology and determine routes based on Dijkstra's shortest path algorithm, for example.

\subsubsection{RGR}

The Reactive-Geographic hybrid Routing $[8,15,16,20]$ protocol is obtained by merging AODV with the Geographic Greedy Forwarding (GGF) [20,21] protocol. The very high mobility of the nodes and the limited transmission range lead the routes to break quite often in AODV. When that happens, AODV optionally offers a route repair mode. In RGR, this route repair is replaced by GGF for an improved performance. When a link in a route breaks while a packet is trying to be transmitted to the next hop, the packet is sent to the neighbor that is closer to the destination. In RGR, as inherited from AODV, nodes periodically broadcast HELLO messages so that neighbors can update their respective routing tables. This combination of AODV and GGF yielded the RGR protocol. A reliability criterion [16] is made use of during the route discovery phase, and a recovery strategy [15] deals with GGF failures. The goal of the reliability criterion is to select the most robust and reliable route from the route discovery. This is achieved by making use of the concept of reliable distance [22]. As far as GGF is concerned, the idea is to forward the data packet to the neighbor whose location is closer to the destination than the holding node, just as in the greedy forwarding algorithm of GRP. If there is no such neighbor, GGF is said to have failed, and the packet is dropped. To overcome this failure, a lowcomplexity and low-overhead recovery strategy, unlike the perimeter mode of GRP's greedy forwarding, consists of forwarding the packet to the Best-Moving Node (BMN) when GGF fails. The BMN is the node, within the transmission range (the holding node included), that is deemed to move faster toward the destination. The decision on which node is moving faster toward the destination is based on predictions that are made using the current speed and direction of the nodes.

\subsection{Antenna Model}

We model a switched-beam directional antenna with $N$ separate antennas that collectively cover $360^{\circ}$. If for instance we choose $N=6$, then each antenna will have a beamwidth of $60^{\circ}$. Likewise, the beamwidth will be $90^{\circ}$ for $N=4$, etc. Broadcasting is performed by sequential transmissions on all beams, one beam 
at a time, as described in [23]. Each beam represents a distinct antenna sector. Therefore, we use the names beam and sector interchangeably in these pages. For a 6 -beam antenna $(N=6)$, we have the configuration shown in Figure 2.1 with a beamwidth of $60^{\circ}$. The front in the figure represents the direction of movement of the node. The beams are numbered in ascending order counterclockwise, with the front being the reference point/line.

Let us assume a free space propagation model and the use of the Friis transmission equation (Equation 2.1) to set/derive parameters such as Tx/Rx power, transmission range, and antenna gains. We have:

$$
\frac{P_{r}}{P_{t}}=G_{t} G_{r}\left(\frac{\lambda}{4 \pi R}\right)^{2}
$$

where: $P_{r}=$ the received power, $P_{t}=$ the transmitted power, $G_{t}=$ the transmitter's gain, $G_{r}=$ the receiver's gain, $\lambda=$ the wavelength, and $R=$ the transmission range. Let us consider an omnidirectional antenna scenario for instance. In OPNET [14], setting the parameters as follows will result in a transmission range of $1000 \mathrm{~m}: P_{r}=$ $3.2 \mathrm{~mW}, P_{t}=-95 \mathrm{dBm}, G_{t}=G_{r}=0 \mathrm{~dB}$, Frequency $=2.4 \mathrm{GHz}$. This resulting $1000 \mathrm{~m}$ of transmission range gives a circular transmission coverage area. For fairness in comparison, we are setting the coverage area to be the same for both omnidirectional and directional antennas. In the case of the directional antenna, the coverage area is obviously not going to be circular anymore. Rather it will take the shape of one of the antenna beams shown in Figure 2.1, with a longer radius (transmission range). Let $r_{0}$ be the radius for the omnidirectional case, and $r$ the radius for the directional case. The omnidirectional antenna covers $360^{\circ}$, whereas the directional antenna covers $60^{\circ}$ beamwidth. We want the two coverage areas to be equal, hence:

$$
\frac{60}{360} \pi r^{2}=\pi r_{0}{ }^{2} \Rightarrow r=r_{0} \sqrt{6}
$$

With $N$ being the number of antenna sectors, Equation 2.2 generalizes to Equation 2.3 as follows:

$$
\frac{360 / N}{360} \pi r^{2}=\pi r_{0}^{2} \Rightarrow r=r_{0} \sqrt{N}
$$

As mentioned earlier, we considered a radius of $1000 \mathrm{~m}$ for the omnidirectional case. 


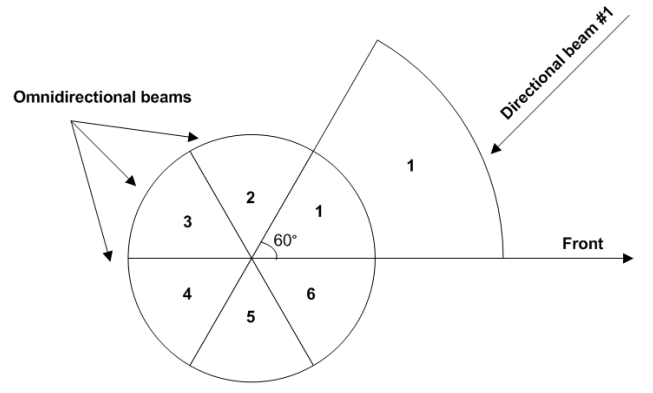

Figure 2.1: Antenna Beams Configuration

Therefore, in the 6-beam directional case, we need a radius of $r=100 \mathrm{Q} \sqrt{6}=2449 \mathrm{~m}$. All the other parameters remaining the same, we need to find the gain of the transmitter antenna $\left(G_{t}\right)$ that gives us such a radius. By rearranging Equation 2.1, we find $G_{t}=6$, which is about $7.8 \mathrm{~dB}$. Therefore, we are going to have a directional antenna of gain $7.8 \mathrm{~dB}$ for a beamwidth of $60^{\circ}$. A different beamwidth would give a different gain that would be obtained following the exact same steps as above.

The antenna has two modes: a directional mode and an omnidirectional mode.

- Directional Mode: only one sector (beam) is active at a time. The others are deactivated. We deactivate a beam simply by setting its gain to $-200 \mathrm{~dB}$. We set the gain of the active sector so that the transmission range is extended. This activate/deactivate scheme is also used in [13]. When a node wants to transmit a frame, it goes into directional mode. A receiver node turns towards the sender node when a sequence of packet exchanges (such as RTS-CTS-DataACK in IEEE 802.11) is initiated, and returns to omni mode (idle state) at the end.

- Omnidirectional Mode: all $N$ sectors are active with a gain of $0 \mathrm{~dB}$. However, only one sector is allowed to receive at a time. Receiving simultaneously on two or more sectors is treated as a collision. A node continuously stays in this mode unless it is engaged in an RTS-CTS-Data-ACK exchange. Transmission is forbidden in this mode. This mode is only entered into in idle mode. All transmissions are performed in directional mode only. 


\subsection{Directional MAC Protocols}

\subsubsection{Key Challenges of MAC Protocols in Radio Networks}

Some key challenges that need to be met in the design of a MAC protocol for wireless networks are as follows.

- Hidden-Terminal Problem: a given scenario experiences the hidden-terminal problem when transmissions from two nodes which cannot hear each other collide at a third node. In the context of directional antennas, all nodes that are located within the destination node's coverage area and are away from the source node's coverage area are hidden terminals. The shaded area $A_{h}$ in Figure 2.2(a) indicates the area in which hidden terminals may exist, from the perspective of node $S$. A node located at any other area where it cannot hear $S$ is not a hidden terminal because, even if it points toward $D$, its signal either will reach $D$ from a direction where $D$ 's antenna is not active or it will be undetectable by $D$.

- Deafness Problem: a source node experiences the deafness problem when it fails to communicate with its intended destination node which is pointing toward a different direction for transmission or reception. Figure 2.2(b) shows node $S$ being "deaf" to a transmission that comes from node $E$.

- Exposed-Terminal Problem: a node experiences the exposed-terminal problem if it assumes a busy medium and defers its transmission even though it could be transmitting without impeding ongoing transmissions by other nodes.

- Broadcasting: a node performs broadcasting when a packet has to be sent in all directions. A broadcasting scheme needs to be carefully defined in the context of directional antennas.

\subsubsection{Proposed Directional MAC Protocol Design}

MANETs, hence SBA-MANETs, are typically bandwidth limited. Consequently, we can simply ignore at this point all multichannel and busy-tone solutions/mechanisms that are proposed in the literature. Our goal here is not to design the best directional 


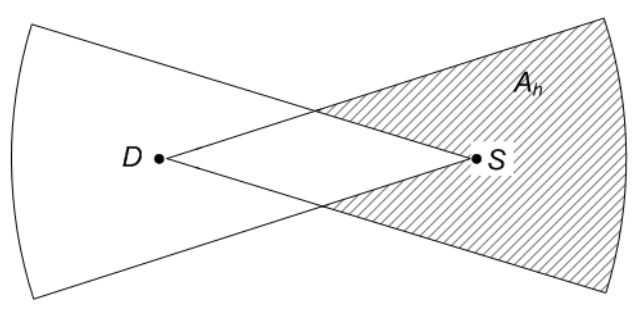

(a) Hidden-Terminal

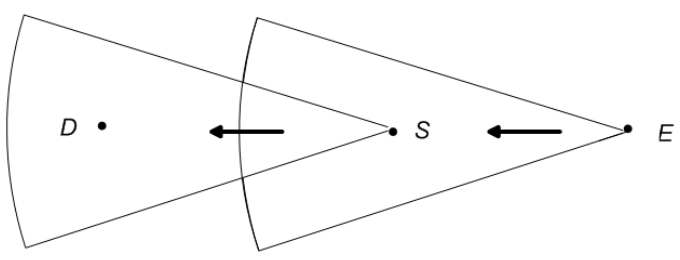

(b) Deafness

Figure 2.2: Hidden-Terminal and Deafness

MAC protocol that exists. Rather, we want a simple SBA-MANET-applicable protocol that captures key features that are common to most state-of-the-art MAC protocols [23-27]. These features address the main challenges inherent to the use of directional antennas: hidden-terminal problem, exposed-terminal problem, deafness, and, to a certain extent, an efficient broadcasting algorithm. We designed these features by adapting the existing IEEE 802.11b DCF MAC protocol.

The IEEE 802.11b DCF MAC protocol (omnidirectional) is contention-based. It employs a CSMA/CA mechanism by means of the Distributed Coordination Function (DCF). We adapt this standard MAC protocol to work with directional antennas. We call the new adapted MAC protocol the IEEE 802.11b-based Directional MAC (DbMAC) protocol. DbMAC works mostly like the standard IEEE 802.11b DCF MAC protocol but on a per-antenna-sector basis. Figure 2.3(a) shows a simplified general operation diagram, the emphasis being put on unicast operation. Given that we are now working on a per-sector basis, broadcast and unicast can no longer operate the same as in the original IEEE 802.11b MAC protocol. The bifurcation is shown in Figure 2.3(a). The broadcasting operation diagram is shown in Figure 2.3(b) and discussed in more detail later.

\subsubsection{Broadcasting}

To broadcast a frame, the node will make copies of that frame and then send them, one at a time, using a different antenna sector for each. Broadcasting is done in either one round or two rounds. When a frame is to be broadcast, the node checks if the medium is free (has been free for at least DIFS) on the first beam. If the medium is 


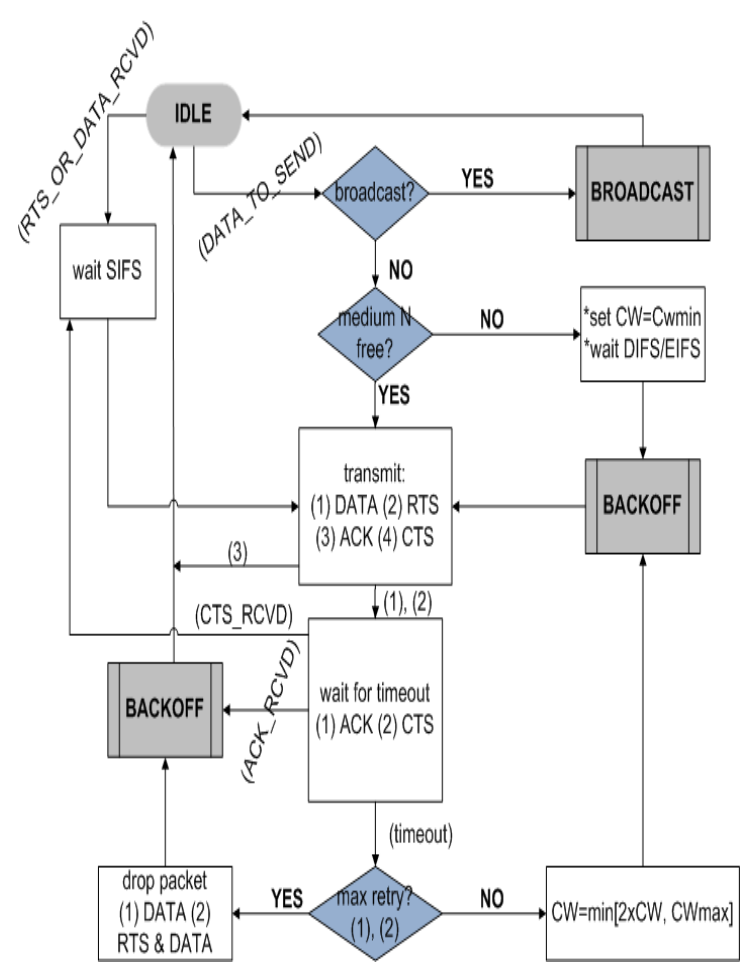

(a) General Operation

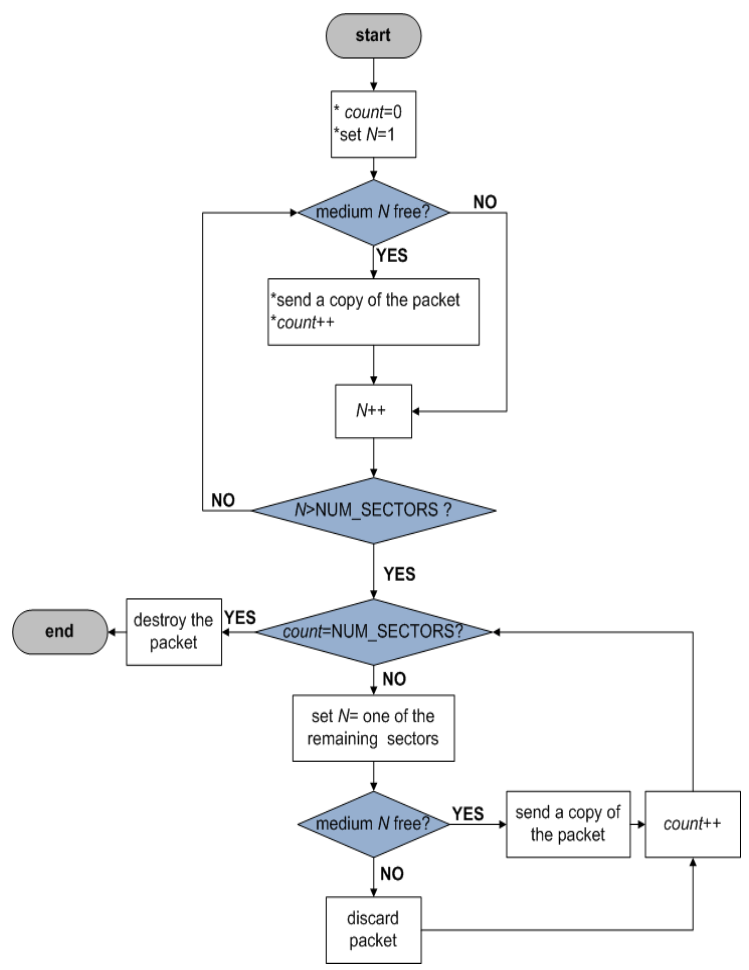

(b) Broadcasting

Figure 2.3: Simplified DbMAC Flowchart

free on that beam, then a copy of the frame is sent in that beam, then the medium on the next beam is checked and so on. If the medium on all the beams was found to be free when checked, then no second round is needed. But if during this first round the medium is found to be busy on at least one beam, then the second (and last) round is triggered. The second round does not start until the first is completed. If the medium is once again found busy on a beam during the second round, the transmission on that beam is abandoned. Broadcasting is relatively expensive in terms of actual number of transmissions required. Having a third round would add more delay. A simplified broadcast procedure is depicted in Figure 2.3(b) where "medium $N$ " means medium on beam $N\left(N=1,2, \ldots N U M_{-} S E C T O R S\right)$.

\subsubsection{Neighbor Table}

Each node maintains a neighbor table. The neighbor table starts off being empty and is progressively populated as follows. Whenever a node receives a frame from the Physical layer, it updates its neighbor table by adding the address of the sender of 
that frame and the antenna sector it arrived on. That way, if a frame is later to be sent to that neighbor we now know which antenna sector to use. Therefore, we do not have to calculate the direction of arrival; we know it as soon as the frame arrives on a given sector (that represents a good-enough approximate direction).

\subsubsection{RTS/CTS}

RTS and CTS are sent directionally. A node first picks a sector that points toward the intended destination of the RTS/CTS.

\subsubsection{DNAV}

The Directional Network Allocation Vector (DNAV) is similar to the NAV described in the original IEEE 802.11b DCF MAC; except now the NAV is kept on a perantenna-beam basis.

\subsubsection{Hidden-Terminal Problem}

The RTS/CTS mechanism from the original IEEE 802.11b DCF MAC protocol helps mitigate the hidden-terminal problem. However, it does not completely solve it. In our context of directional antennas, nodes in $A_{h}$ (Figure 2.2(a)) may initiate transmissions during the time the source node $S$ transmits the RTS, and these transmissions will collide with that RTS at $D$. Once the RTS eventually goes through (thanks to the backoff mechanisms upon collisions), a CTS is issued. Upon receiving that CTS, the nodes in $A_{h}$ will defer, thus solving the hidden-terminal problem for that round.

\subsubsection{Deafness Problem}

We combat the deafness problem as follows. A node only stays in directional mode for the length of a single data packet transmission, from the sending of RTS to the receiving of the ACK. As soon as the ACK for the sent data packet is received, the node returns to omnidirectional mode. For example in the scenario depicted in Figure 2.2(b), node $S$ is initially in omnidirectional mode. To send a data packet to $D, S$ beamforms (directional mode) towards $D$ and first (if need be) sends an RTS to $D$. $D$, which is initially in omnidirectional mode as well, hears the RTS and beamforms towards $S$ in order to send the CTS. Once $S$ receives the CTS, it sends the data packet to $D$. $D$ receives the data packet and acknowledges it by sending an 
ACK back to $S$. As soon as $D$ sends the ACK, it goes back to omnidirectional mode (idle mode). Likewise, as soon as $S$ receives the ACK, it goes back to omnidirectional mode. This allows $S$ to be able to listen to node $E$, thus reducing the Deafness problem. The Deafness problem does not go away completely though. In fact, while $S$ is engaged in an RTS-CTS-Data-ACK transaction with $D$, it may happen that $E$ sends one or more RTS toward $S$ that obviously will not be answered until $S$ is back into omnidirectional mode. These unanswered RTSs contribute to the overhead. Given that there is a limit on the number of retries of the RTS (the Short Retry Limit), $E$ will eventually give up trying if $S$ does not go back to omnidirectional mode before that limit is reached.

\subsubsection{Exposed-Terminal Problem}

The exposed-terminal problem is taken care of through the directional (per sector) NAV times. Only the sector that receives the unintended RTS/CTS will be "blocked" for transmission for the duration of the neighboring/overheard transmission. Any other sector can engage in a concurrent transmission/reception.

\subsection{Simulation and Analysis}

Each number of sectors or each beamwidth that we choose gives us a corresponding transmission range. In fact, the transmission range increases when we increase the number of sectors (see Section 2.3). Thus far, in all of our previous work $[8,15,16]$, we have been working with scenarios of 30 nodes with omnidirectional antennas in an area of $2000 \times 4000 \mathrm{~m}^{2}$. Basically, with this area and given the transmission range of $1000 \mathrm{~m}$, we have 2 hops along the x-axis and 4 hops along the y-axis. For fairness in comparison, we are keeping this same scale for any type (in terms of its number of beams) of directional antenna. Table 2.1 shows the area corresponding to each type of antenna.

We are using OPNET Modeler 16.0 for the simulations. The channel capacity is set to $11 \mathrm{Mbps}$ for all mobile nodes. The rest of the simulation settings and parameters are summarized in Table 2.2. A single traffic flow (one source node and one destination node) for data packets is considered. The 28 remaining nodes 
Table 2.1: Beamwidth-Dimension Matching

\begin{tabular}{cccc}
\hline Number of sectors & Beamwidth & $\begin{array}{c}\text { Transmission } \\
\text { range }(\mathrm{m})\end{array}$ & $\begin{array}{c}\text { Area dimensions } \\
\left(\mathrm{m}^{2}\right)\end{array}$ \\
\hline \hline 1 (omni) & $360^{\circ}$ & 1000 & $2000 \times 4000$ \\
2 & $180^{\circ}$ & 1414 & $2828 \times 5656$ \\
3 & $120^{\circ}$ & 1732 & $3464 \times 6928$ \\
4 & $90^{\circ}$ & 2000 & $4000 \times 8000$ \\
6 & $60^{\circ}$ & 2449 & $4898 \times 9796$ \\
12 & $30^{\circ}$ & 3464 & $6928 \times 13856$ \\
\hline
\end{tabular}

are potential forwarding nodes. For each protocol and with the afore-mentioned set of parameters, we generate 5 independent scenarios using 5 different seeds of the pseudo-random number generator available in OPNET. By doing so, we have 5 sets of pseudo-independent results. These 5 results are then averaged and the $95 \%$ confidence intervals determined and shown in the figures (as vertical lines). These confidence intervals serve to establish the statistical significance of the difference between any two plots.

For the mobility, we consider the Random Waypoint (RWP) model [28, 29]. We are going to model mobility with RWP for the remainder of this work. As pointed out in [8], RWP is frequently used for simulations in MANETs mainly because of its relative simplicity and wide availability in simulators. It works as follows. A node randomly picks a location within the simulation area and moves to that location in a straight line, using a randomly chosen speed. Upon arrival at that location, the node pauses and picks another location and speed. When the pause time is set to 0 , the node never stops until the simulation is over; it keeps randomly picking a new location to move to without pausing. Figure 2.4 shows the simulation trajectory trace of a mobile node under RWP in a $2000 \mathrm{~m} \times 4000 \mathrm{~m}$ area. With RWP, the nodes move independently, in different directions. Therefore, even though we assume 2D networks and the speed range is the same for all the nodes and is relatively tight, the nodes are still fairly mobile with respect to one another. They do not move as a fixed swarm. The protocol design would be simplified were the nodes moving as a fixed swarm, since their relative speed with respect to one another would be very 
Table 2.2: Simulation Parameters

\begin{tabular}{ll}
\hline Parameter & Value \\
\hline \hline Number of Simulated Nodes & 30 \\
Area Dimensions & variable \\
Transmission Range & variable \\
Packet Size & 1024 bits, average \\
Data Rate of the Channel & $11 \mathrm{Mbps}$ \\
Traffic Sent Rate & 5 pkts $/ \mathrm{s}$ \\
Mobility Model & Random Waypoint \\
Speed of Nodes & $50-60 \mathrm{~m} / \mathrm{s}$ \\
Pause Time & $0 \mathrm{~s}$ \\
Simulation Duration & $1800 \mathrm{~s}$ \\
HELLO Interval for all protocols & $1 \mathrm{~s}$ \\
GRP Quadrant Dimensions & $2000 \times 2000 \mathrm{~m}^{2}$ \\
GRP Distance Moved Limit & $1000 \mathrm{~m}$ \\
OLSR TC Interval & $5 \mathrm{~s}$ \\
AODV TTL Threshold & 10 \\
AODV Net Diameter & 20 \\
\hline
\end{tabular}

small if any. But in this work we are interested in designing a protocol that will work in the worst case of independently moving nodes.

In order to work with a high-enough percentage of sent packets that have used two or more hops to be successfully delivered (a metric dubbed as the Amount of Routing in [12]), we focus on a specific area size and explore how the protocols operate as a function of the number of sectors. More specifically, we base this analysis on the $4898 \times 9796$ area, with most attention paid to the performance of the 6-beam antennas.

In terms of PDR, we can see from Figure 2.5(a) that GRP consistently shows a very low PDR compared to the other three protocols regardless of the beamwidth 


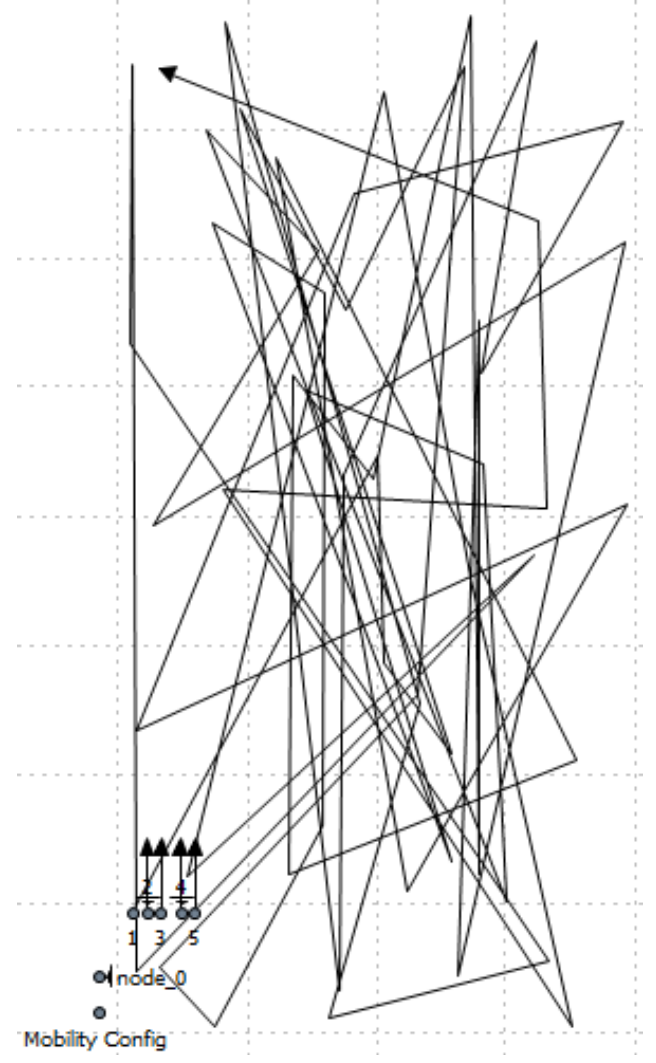

Figure 2.4: Random Waypoint Trajectory Example

that is used for directional antennas. The gap is less apparent with omnidirectional antennas. Focusing on the 6 -beam $\left(60^{\circ}\right.$ beamwidth) antenna, we can see that, while GRP has the worst PDR, RGR and OLSR have the best PDR and AODV is in between. An early conclusion might then suggest that it is better to use either RGR or OLSR as a routing protocol for SBA-MANETs in terms of achievable PDR. Note that the PDR is pretty close in other numbers of sectors, but we do not consider those because the considered area is not adapted for those sector numbers. Recall that to each number of sectors corresponds a different transmission range. For example, when the number of sectors is 12 , routing barely happens because the transmission ranges are so long that routing barely happens, hence the results do not really reflect the routing mechanisms. Focusing on the 6-beam antenna still, we can also see that there is no noticeable difference among the protocols in terms of latency (see Figure 2.5(b)). The latency is about $100 \mathrm{~ms}$ for all. Note that this latency is higher when the number of sectors is less than 6 . That is because the network is sparser and it takes more time to discover routes. Plus, routes break more often and it takes more effort (switches 


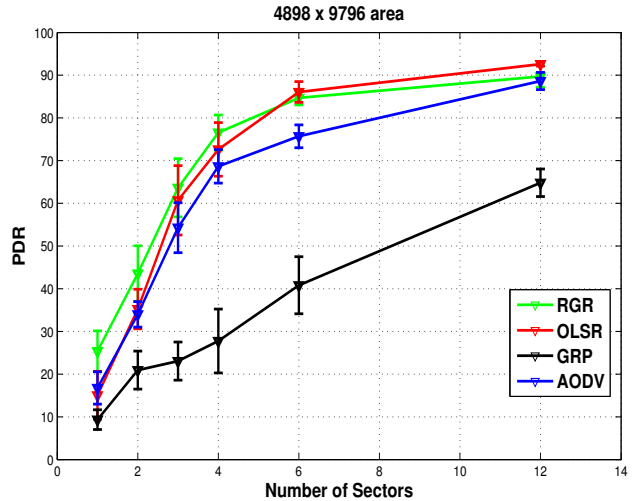

(a) Packet Delivery Ratio

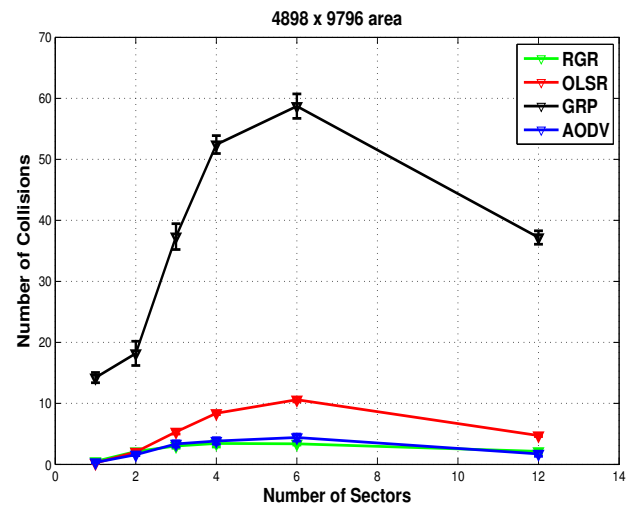

(c) Number of Collisions

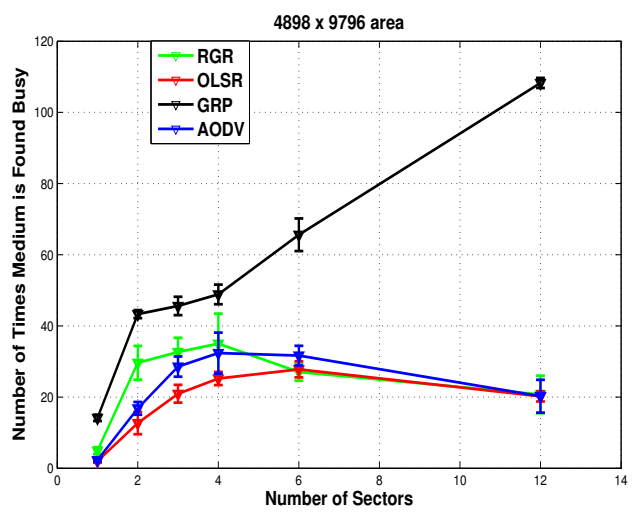

(e) Busy Medium

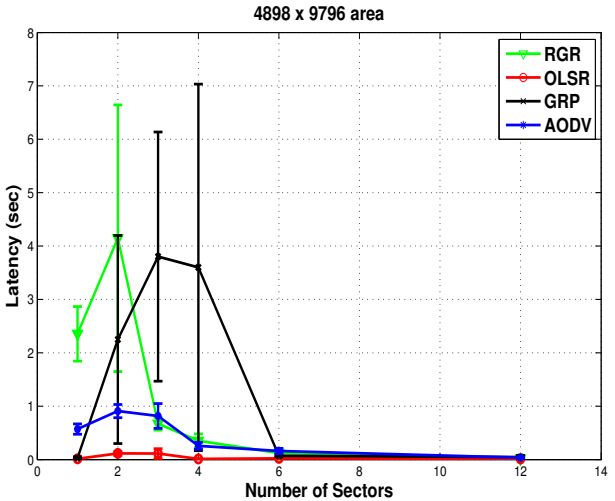

(b) Delivered Packets Latency

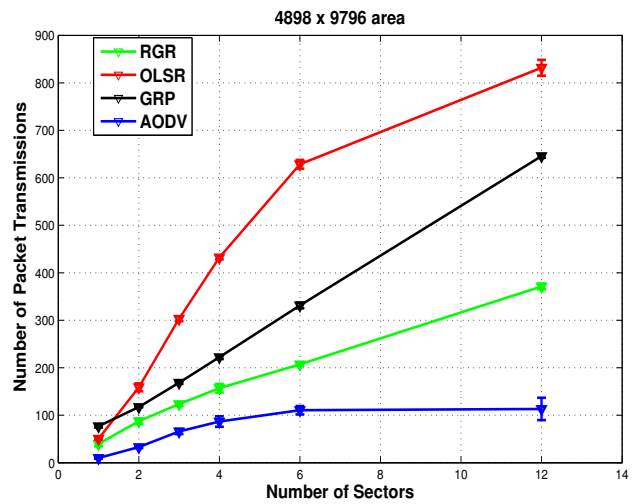

(d) Number of Transmissions

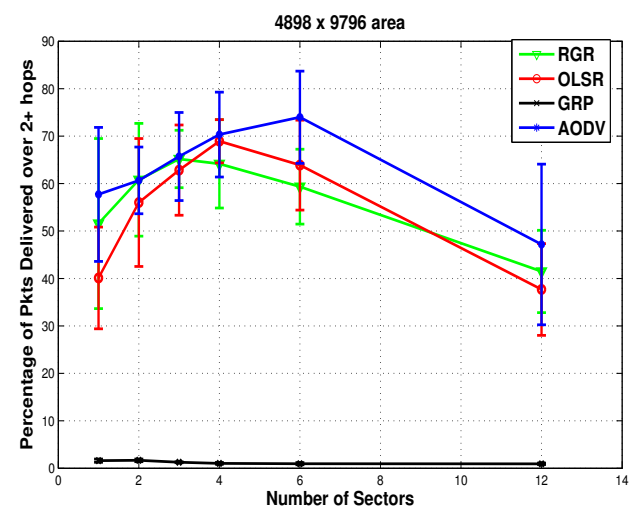

(f) Percentage of Packets Delivered in 2+ hops

Figure 2.5: Routing Performance

to GGF, etc.) and therefore time for successful packets to reach the destination. 
The relatively low PDR of GRP can be explained as follows. The high mobility in some SBA-MANETs (such as SBA-FANETs) results in more flooding messages, in addition to the HELLO messages, being sent out. In GRP, whenever a node moves a certain preset distance or crosses a quadrant, it must flood (broadcast) its new position to all the nodes present in that new quadrant. So there is a lot of broadcasting that is associated with GRP. In fact, RGR, AODV, OLSR, and GRP respectively have 368, 376, 1319, and 1755 broadcast occurrences for 30 nodes in 1800 seconds of simulation. Due to the extended transmission ranges in our directional antennas, GRP's flooding messages reach nodes that are beyond the node's quadrant, rendering the medium busy to those nodes that belong to other quadrants. Collisions then ensue because these other nodes also have their own transmissions to make as a result of, among other reasons, moving out of their own quadrant or moving a certain distance. We can therefore see from Figure 2.5(c) that GRP also stands out in terms of collisions. Note that, in Figure 2.5(c), there is a peak when the number of sectors is 6 . The explanation is as follows. As shown in Table 2.1, $4898 \mathrm{~m} \times 9796 \mathrm{~m}$ is the "optimal" area corresponding to 6-sector antennas, in the sense that it is with that area (among all the areas considered) that the most multi-hop routing happens with 6-sector antennas. A smaller number of sectors (shorter transmission range) makes for a sparse network, which results in less collisions. On the other hand, a higher number of sectors (longer transmission range) makes for a network where most nodes are just one-hop away from one another, hence no need for routing; which results in less control packets and therefore less collisions. As a consequence we have the peak when Number of Sectors is 6. In terms of the number of transmissions, although GRP shows a relatively high number of packet transmissions, it is second to OLSR overall (see Figure 2.5(d)).

The high broadcasting requirement does not necessarily translate into the highest number of transmissions for GRP because broadcasts are not always completed. As described earlier, in our broadcast design the copy of the packet to be sent on a given sector is dropped if the medium is found to be busy on that sector twice. Figure 2.5(e) shows the number of times (per second) that the medium is found busy in an attempt for unicast or broadcast. And as we can see in the figure, the medium is found busy more frequently with GRP. 
Furthermore, Figure 2.5(f) shows that consistently less than $3 \%$ of packets delivered using GRP are delivered in 2 or more hops. This means that over $97 \%$ of the delivered packets are delivered in just one hop, which means that there is not much routing going on with GRP at all. Basically, whenever the destination is not located one single hop away from the source, the packet is most likely not delivered. Failed broadcasts make the available information about a destination's location inaccurate. Added to that are the many collisions that occur. These two reasons make the delivery along longer paths very unlikely. In a nutshell, GRP, as it stands, performs very poorly in SBA-FANETs mainly because of the fact that broadcasting is required more often, which results in a lot of collisions. A way of fixing this will be to make both the quadrant dimensions and the preset distance limit dependent on the beamwidth (thus the transmission range) used. And this would be cross-layer information sharing. We might also revisit our broadcasting scheme to some extent.

Even though OLSR shows good performance in terms of PDR and latency, this comes at the expense of a relatively high overhead in terms of number of transmissions as we can see in Figure 2.5(d). This comes as no surprise given the fact that OLSR is a proactive routing protocol that requires frequent TC messages in addition to HELLO messages, all of which are broadcast messages. However, broadcasting in OLSR is less frequent than in GRP, resulting in less "jamming" of the medium; which in turn results in less collisions and less instances of the medium found busy. Nevertheless, with OLSR we still have significantly more collisions than in AODV and RGR.

As already mentioned, RGR, along with OLSR, show the best PDR. RGR comes with significantly less overhead than OLSR in terms of transmissions required. We can therefore at this point see that RGR positions itself as the best protocol for SBAMANETs. However, the latency for RGR is still significantly high, on the order of $100 \mathrm{~ms}$.

\subsection{Summary}

In this chapter, we have investigated the benefits of using single-beam directional antennas in MANETs. We have done so by comparing the performance of existing 
routing protocols in MANETs when directional antennas are used. In order to use directional antennas, we first designed a generic directional MAC protocol for SBAs. We then evaluated the following four routing protocols in SBA-MANET scenarios: AODV, OLSR, RGR, and GRP.

The key lessons from our analysis are: (i) Directional antennas are beneficial, as they allow us to cover larger areas with the same number of UAVs; (ii) If we are to pick a suitable routing protocol as a starting point in SBA-MANETs, it should be RGR; and (iii) Latency is an issue even when we use the best possible routing protocol thus far (RGR).

To go beyond the current level of performance of RGR and reduce the latency, we are going to explore, as our next step, the benefits of using the multi-beam capabilities offered by modern directional antennas. Specifically, with delay-sensitive applications in mind, we are going to investigate, by means of an optimization model, how those multi-beam capabilities can allow us to significantly reduce the end-to-end delay (latency) while dealing with not just one but multiple traffic flows. In this chapter we have only considered one flow; which is not very realistic inasmuch as a typical MANET or FANET mission is expected to require multiple traffic flows. Ultimately, we will have to design a MAC protocol for MBA, and propose a routing protocol that will reduce the end-to-end delay by exploiting MBA capabilities. Given the promising results that RGR has shown in this chapter with single-beam antennas, it positions itself as a strong candidate protocol to build on.

The rationale for exploring MBAs as the next step is pretty straightforward: if single-beam directional antennas have shown so many benefits in MANETs, MBAs ought to bring about even more benefits and performance improvements. In the next chapter, we present MBAs and we review the literature in order to have a clear idea of how and/or to what extent their capabilities have been exploited in MAC and routing protocols for MANETs/FANETs. 


\section{Chapter 3}

\section{Multi-Beam Antennas: Description and Literature Review on Design and Performance}

\subsection{Introduction}

As already stated, our ultimate goal is to propose a comprehensive routing solution that capitalizes on the full Multi-Packet-Transmission/Multi-Packet-Reception (MPT/MPR) potential of MBAs to minimize the E2E delay of delivered packets in multi-flow ad hoc networks, including MANETs and FANETs. This chapter is evidence that such a solution is yet to be proposed. In order to have a broad view of the relevant state of the art, this chapter reviews the literature that pertains to MBAs and/or performance optimization in wireless networks. Note that Multi-Packet-Transmission/Multi-Packet-Reception is also known as ConcurrentPacket-Transmission/Concurrent-Packet-Reception (CPT/CPR). We use both terms interchangeably throughout this document.

More specifically, this chapter shows that: a) MBAs have been widely utilized in wireless (both infrastructure and infrastructureless) networks mostly to improve capacity and throughput; b) little work has been done to exploit the MPT/MPR

potential of MBAs to minimize the E2E delay. Furthermore, we point out a popular methodology used in optimizing link scheduling proposals aimed at various performance improvements. The methodology consists of formulating a linear-programming problem for optimal solutions and then designing a heuristic protocol accordingly. We 
shall make use of this methodology to a significant extent in our current work.

\subsection{Design of Multi-Beam Antennas}

MBA techniques for MANET applications are discussed in [7]. MBAs can be implemented either in the form of Multiple Fixed-Beam directional Antennas (MFBAs) or in the form of Multi-Channel Smart Antennas (MCSAs). To form multiple fixed beams, MFBAs and Multiple Radios (MRs) with a directional antenna on each radio (transceiver) can be exploited [30,31]. As a result, high network throughput can be achieved. In a stationary environment, the antenna patterns can be optimized to further improve network performance. However, the performance of MFBAs/MRs degrades in a time-varying multipath propagation environment, which is typically experienced in indoor and low-altitude outdoor wireless networks [32]. As already mentioned, the other approach to implement MBAs is to use MCSAs [33-35]. By using smart antenna techniques, multiple beams can be adaptively and dynamically formed by a node so as to provide robust communication links with multiple users. At the expense of higher complexity, an MCSA-based approach provides the same advantages as the MFBA/MR design, but its performance does not degrade in time-varying multipath environments [34,35]. Many MANETs, such as FANETs for example, are typically outdoor and relatively high altitude, therefore MFBAs/MRs can be used without worrying about the performance degradation inherent to timevarying multipath propagation environments. This also spares us the complexity of MCSAs altogether.

In MFBAs, multiple active beams can be selected from the predefined beams, whereas in MRs, each radio is equipped with its own predefined directional antenna [30]. Both directional structures achieve concurrent communications with multiple users in addition to inheriting the advantage of the switched-beam antennas.

Multiple channels are assumed in [7] for the multi-beam structures. Basically, each beam has its own channel. We are not going to assume multi-channel structures here, as MANETs are typically limited in bandwidth, as pointed out in [36, 37]. For all the reasons above, as well as for limited complexity, we are going to use the MFBA/MR technique to realize MBAs, with a single channel for all beams. 


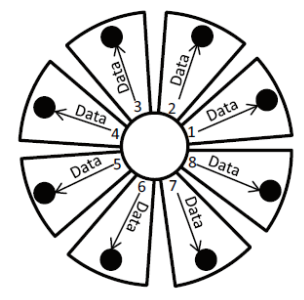

(a) Omni mode

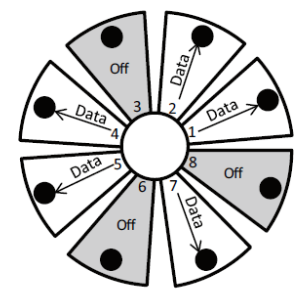

(b) Tx mode

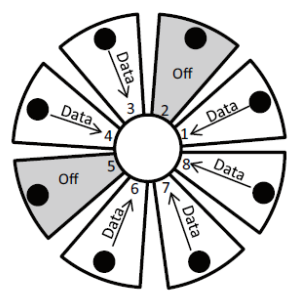

(c) Rx mode

Figure 3.1: Multi-Beam Antenna Modes [1] (C2016 IEEE

Furthermore, for the same reason of limited complexity, we are going to only consider 2D networks in this work. In real life though, the pitch, roll, and yaw of the UAVs (in the case of FANETs) will have to be taken into account in 3D scenarios. This would complicate the computations, but the main ideas of the proposed protocol should not change.

In a nutshell, with fixed-beams and single-channel in mind, an MBA system can be pictured as a set of directional radio transceivers all sharing the same channel. These transceivers are facing different directions to prevent signal interference with each other. MBA nodes obey an important transmission/reception rule due to their half-duplex operation [1]: an MBA node cannot transmit signals in some beams and receive signals in other beams at the same time. At a given time, an MBA node makes all its beams operate in either transmission or reception mode (see Figure 3.1).

When MBAs are employed, more sophisticated MAC and routing mechanisms are necessary in order to exploit spatial reuse and control the amount of interference and collision. The discussion in [7] focuses on the space-domain approaches, whereas other works such as [38] involve the design of frame structures in the time domain. Scheduling schemes combining the spatial and time dimensionality may increase the network flexibility and efficiency. Furthermore, cross-layer design might be desirable to yield joint Physical layer, MAC, and routing optimization [39-41]. 


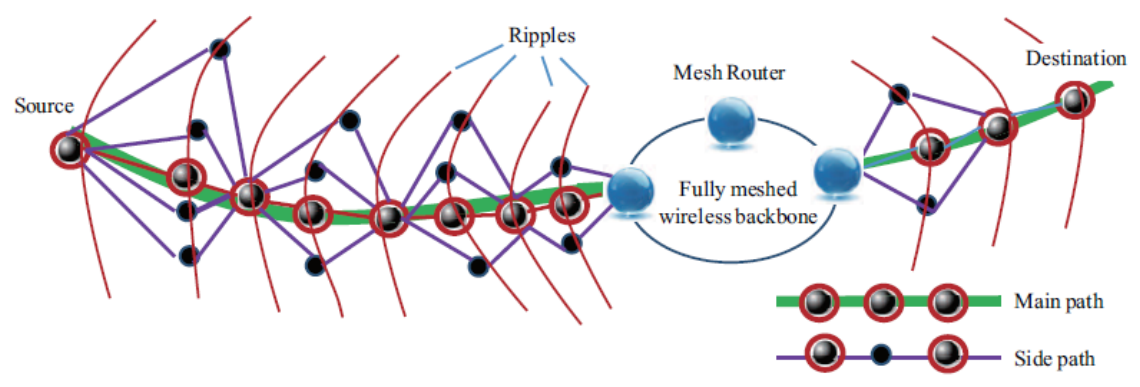

Figure 3.2: Concept of ripple-diamond-chain routing [1] (C2016 IEEE

\subsection{Routing Protocols for Multi-Beam Antennas}

A multi-beam routing protocol based on a Ripple-Diamond-Chain (RDC) formation is proposed in [1]. The cross-layer routing scheme aims to fully exploit the potential of MBAs by improving the utilization rate of beams. A prominent feature of RDC routing is that it establishes a few side paths around the main path, in order to utilize the MPT/MPR capability. The design assumes static Wireless Mesh Networks (WMNs) where some nodes, called mesh routers, form the backbone and others are mesh clients. In effect, nodes belonging to the same ripple have the same number of hops to the mesh router. Ripple IDs can be easily determined through an ad hoc network routing protocol such as Dynamic Source Routing (DSR). Ripples look like water waves (see Figure 3.2): when one ripple is sending data, the next ripple can only receive data. However, the 2-ripple away nodes can also send data to achieve pipelined transmissions. Therefore, besides the diamond chain formation process in the routing layer, the authors also propose a ripple-to-ripple localized transmission schedule control scheme, which belongs to the MAC layer. The topology (with fixed mesh routers) that this protocol is designed for can be assimilated to an infrastructure network to some extent.

Uddin et al. [42] present a cross-layer formulation for joint routing, scheduling and spectrum allocation in a WMN with variable channel widths. They show that although narrower spectra result in more orthogonal channels each with larger transmission range, such narrow bands will result in small capacity links and limit the spatial reuse of the same spectrum block across the network. They also show that wider spectra result in larger capacity links and better spatial reuse, but also 
result in smaller transmission ranges, resulting in more hops along end-to-end routes and hence exacerbate the intra-path interference problem. The problem the authors solve is equivalent to minimizing the system activation time in delivering multiple sessions without violating the minimum SINR requirement for communications.

\subsection{MAC Protocols for Multi-Beam Antennas}

Designing MAC protocols specifically for MBA-equipped nodes and networks is important because traditional MAC protocols based on the IEEE 802.11 standard are designed to work with omnidirectional and single-beam directional antennas, and therefore cannot take advantage of the unique capabilities of MBAs as they do not facilitate concurrent transmissions or receptions by a node. Broadly, MAC protocols for MBAs can be categorized in two groups: centralized protocols and distributed protocols. Centralized protocols are for infrastructure networks where usually only the infrastructure (access point or base station) is equipped with an MBA. For infrastructureless (such as ad hoc) networks where all the nodes are equipped with MBAs, we have distributed protocols.

\subsubsection{Centralized Protocols}

In this section, we explore MBA MAC protocols that are proposed for infrastructure networks. For instance, Dong and Petropulu [43] combine the idea of Multi-Beam Adaptive Arrays (MBAA) with a cooperative medium access protocol for cellular networks. In the event of a collision, and during the collision slot, the MBAA will adjust the array weights to form multiple beams focusing on packets coming from different directions. Each beam that contains a single packet will be used to recover that packet. If there is at least one beam that contains multiple non-resolvable packets, a Cooperative Transmission Epoch (CTE) will follow the collision slot. During each slot of the CTE, multiple relays are selected in a predetermined order to forward the mixtures that they received during the collision slot. These mixtures arrive at the MBAA at different angles, and thus provide independent equations involving all the collided packets. Once enough equations, from both the collision slot and the CTE slots, are collected, the collided packets are recovered by formulating and solving a MIMO problem. The proposed approach reduces the effective collision 
order and the duration of the CTE, and thus results in a significant throughput improvement at the expense of additional hardware cost at the base station.

Very similar to the work by Dong and Petropulu [43], Li et al. [44] propose another modified cooperative medium access protocol with the use of MBAAs. All the beams formed in the collision slot as well as the CTE slots are exploited to retrieve the packets, and the number of relays in each CTE slot can be up to the number of antennas at the Base Station/Access Point (BS/AP). Spatial signature-based, instead of Angle-of-Arrivals (AoAs)-based, collision detection is used. Furthermore, two relay selection schemes are developed. One is to choose relay nodes with high channel gain to the BS/AP antennas, whereas the other further ensures that the selected relay nodes have low spatial correlation coefficients. Simulation results show a relatively high throughput performance of the proposed protocol and benefits due to the use of the relay selection schemes. The throughput improvement is more significant when the network has a high load.

In [45], Wang et al. propose a CSMA/CA-based uplink MAC protocol for wireless LANs with MBA access points. Spatial reuse is utilized by allowing as many parallel uplink data transmissions as possible in order to improve the throughput. Since all the nodes, including the AP, run a CSMA/CA-based MAC protocol, the authors claim that the proposed protocol is not limited to the single-hop case, but can be easily extended to multi-hop ad hoc networks. In [46], the same authors go further to present an analytical model to evaluate the performance of multi-beam wireless LANs. The beam-synchronization problem, the beam-overlapping problem, and mobility issues are also addressed.

In [47], Tang et al. propose a MAC protocol for WLANs with MBA-equipped APs, omnidirectional-antenna mobile nodes and a single frequency channel. The protocol addresses a series of challenging problems such as the beam-load unbalance problem, the unnecessary defer problem, the receiver blocking problem, and the antenna-imperfection problem. By addressing these issues, as many parallel transmissions between terminals and the AP as possible are successfully facilitated and the throughput of the network is improved. However, there is no indication of how this protocol would fare in ad hoc networks. 
George Thomas [48] proposes a dynamic beamforming protocol for base station receive antennas for wireless random access systems. Packet collisions are resolved by successively splitting an initially omnidirectional beam until the interfering mobile transmitters are spatially separated. It is shown that average delays can be substantially lower when compared with the standard Aloha protocol while maintaining the same throughput levels. The average load on the beam signal processor is shown to be extremely low when the new beamforming protocol is used. Designs issues related to spatially overlapping beams are also discussed.

Chou et al. [49] propose a polling-based MAC protocol, named M-HCCA, for a WLAN with multi-beam AP. M-HCCA integrates time-bounded reservation, cross-layer rate adaptation, energy-conserving scheduling, and mobile-assisted admission control into one scheme to support real-time multimedia traffic. Simulation results show a significant improvement of throughput in uneven station distribution, imperfect station's beamforming, and high mobility environments.

A multiple access scheme in aerial sensor networks using MBAs with a spatial reuse protocol is proposed in [50]. The system model consists of two types of UAVs: a master UAV and actor UAVs. The master UAV is placed in the center of actor UAVs and serves as a relay for data transmissions. An adaptive array antenna is used on the master UAV to avoid signal interference, so that packet collisions among cooperative UAVs can be minimized. Based on different antenna models, the authors propose a MAC protocol that adopts spatial reuse to enable simultaneous data transmissions, therefore increasing the system throughput. Inasmuch as only the center UAV is equipped with an MBA, this solution is no different from the use of MBAs only on APs of WLANs already reviewed. Nonetheless, there are some MAC protocol proposals in the literature that are designed for infrastructureless networks where all the nodes are equipped with MBAs. We review those in the next section.

\subsubsection{Distributed Protocols}

A Multi-Beam Uncoordinated Random Access MAC (MB-URAM) for emerging systems capable of adaptive digital beamforming is presented in [51]. MB-URAM 
asymptotically achieves the network capacity upper bound as beamwidth goes to zero, the number of users grows large, and latency requirements are relaxed. The authors also factor in practical considerations on the performance of MB-URAM, including power constraints, latency, beamwidth, and packet error rate. A probability-based analysis is performed showing that MB-URAM still performs well even when realistic constraints are imposed. Numerous algorithms are also proposed to help improve the performance of MB-URAM under these practical constraints. By the authors' own admission, even though adaptive digital beamforming (the MCSA approach) has already been applied to radar systems and development is accelerating for its use in mobile ad hoc communication systems, this approach has high computational complexity. Zhang et al. [7] also made the same claim, adding that its sole advantage over an MFBA/MR approach is that its performance does not degrade in time-varying multipath propagation environment. And since FANETs, for example, are not expected to have a time-varying multipath propagation environment, which is typically experienced in indoor and low-altitude outdoor wireless networks, we see no point in pursuing adaptive digital beamforming. Fully digital beamforming antenna arrays that are capable of adaptive multi-beam communications allow users to form multiple simultaneous transmit or receive beams within the same frequency channel, while adaptively steering nulls to minimize interference with other users. Applying this notion of using the same frequency channel to MFBA/MR systems in MBA-MANETs is an interesting avenue to pursue.

In [2], Jain et al. present a detailed discussion of various issues involved in designing a MAC protocol for MBA-MANETs. Traditional on-demand MAC protocols for omnidirectional and single-beam directional antennas based on the IEEE 802.11 DCF mechanism cannot take advantage of the unique capabilities of MBAs inasmuch as they do not facilitate concurrent transmissions or receptions by a node. The Hybrid MAC (HMAC) protocol is proposed in [2]. HMAC enables MPT/MPR on a node equipped with MBAs, and is backward compatible with IEEE 802.11 DCF. HMAC is a cross-layer protocol that uses information from both the Network and the Physical layers for its operation. HMAC uses a separate queue for each beam to avoid Head-Of-Line blocking. HMAC also uses a scheduling message that is sent in all desired beams other than the ones being negotiated via RTS/CTS. The novel features of HMAC include: its channel access 
mechanism, the algorithms for mitigating deafness and contention resolution, jump backoff and role priority switching mechanisms for enhancing throughput, and its backward compatibility with IEEE 802.11 DCF. Simulation results show that the performance of MBAs largely depends on the network topology. The authors therefore claim that MBAs are more applicable for WMNs and APs in WLANs. Furthermore, using extensive topological and traffic patterns, they demonstrate that employing MBAs and HMAC can result in significant performance improvements in terms of both aggregate throughput and average end-to-end packet delay. In most of the sample topologies, HMAC delivers near-optimal performance. From a study of random topological scenarios, the authors also conclude that both single and multi-beam antennas deliver comparable performance in ad hoc scenarios. But these claims are not based on any formal optimization model of their metrics.

In [52], Verma et al. propose the Multiple-Beam Antenna Array MAC (MBAA$\mathrm{MAC}$ ) protocol which is a distributed, asynchronous and adaptive MAC protocol and works on the single channel and single transmission power architecture. It is based on the IEEE 802.11 standard. It performs exponential backoff with the same contention window size as defined in the IEEE 802.11 standard. To achieve concurrent transmissions, MBAA-MAC has three key features. First, after successfully exchanging the RTS/CTS frames, the transmitter does not send the Data packet immediately as in the IEEE 802.11 scheme. It waits for a time period called Additional Control Gap (ACG), which is inserted between the RTS/CTS and Data packet. This ACG provides the neighboring nodes, in the vicinity of the sender and the receiver, a chance to exchange their own control packets and schedule concurrent data transmissions. Secondly, the MBAA-MAC uses collision avoidance information in the control frames (RTS/CTS) and thereby instructs neighboring nodes in the vicinity to go to silence mode. This information is used by the neighboring nodes of the transmitter/receiver to determine the possibility of scheduling their transmission. Finally, in MBAA-MAC, the concurrent transmission depends on the information overheard by the neighboring nodes in the vicinity of transmitting or receiving nodes. Thus, it is a locally controlled process and therefore an asynchronous one.

The dynamic TDMA-based MAC protocol for directional antennas (NDTDD) is proposed in [53]. Time slots are dynamically allocated for each beam of every node 
without any central coordinator. The time frame is composed of three sub-frames: admission, assignment and reservation sub-frames. In NDTDD, a node can transmit packets in different beams simultaneously (MPT capability). Nothing is said about the MPR capability of the antennas. The coherence of the available transmitting reservation slots set and the receiving reservation slots set of the whole network is maintained by using two-hop topology beam information. The reservation mechanism ensures that all beams get reservation slots fairly according to their traffic. All nodes achieve time synchronization by Frequency-Hopping Spread Spectrum (FHSS) at the Physical level; which implies the use of multiple channels.

A MAC Protocol for Multi-Beam Directional Antennas (MBDMAC) based on the CSMA/CA scheme and a dedicated control channel with a Directional Network Allocation Vector (DNAV) table is proposed in [54] and [55]. Each beam has its own control channel, making the communications among different beams independent. After sensing all the sectors of the MBA, a global assignment strategy is used to assign the directional communication channels. Simulation results show an improvement in throughput.

A novel SIR-based MAC protocol for enabling Multi-Packet Communication (MPC) in heterogeneous IEEE 802.11 networks adopting smart antenna systems is proposed in [56]. The presented solution, which maintains backward compatibility with the IEEE 802.11 standard, is based on a local but accurate estimation of the instantaneous SIR and on the use of LDPC codes to obtain a reliable estimation of the success or failure of the transmission attempt of each active node. The proposed scheme is compared to a previously developed protocol in terms of throughput and fairness in a multi-path fading environment. The derived results show that the SIR-based access policy, being more adherent to the real network conditions and hence more aggressive, provides a higher throughput, while an access scheme based on a load threshold, being more conservative, may be more suitable in the presence of stringent fairness requirements.

Babich et al. [57] discuss the design requirements for enabling MPC in IEEE 802.11 networks by using advanced antenna systems. They propose two MAC protocols, called TAMPC and SAMPC, which are suitable for asynchronous operations 
in distributed and heterogeneous scenarios, where legacy and non-legacy nodes equipped with different antenna systems can coexist. The SIR-based access adopted by the SAMPC scheme can guarantee higher throughput and fairness with respect to the threshold-based access adopted by the TAMPC protocol, at the cost of an increased but acceptable computational burden.

Furtado et al. [58] propose a decentralized MAC scheme to coordinate the access of multiple transmitters adopting an MPR Physical layer. Using a generic model for the Physical layer, they characterize the throughput achieved by the proposed MAC design when both the MAC and the Physical layers are considered. The formal characterization of the throughput is used to optimize the cross-layer (MAC and Physical layers) operation, taking into consideration the features of the MPR Physical layer and the maximum performance achieved with the proposed MAC design.

Lin et al. [59] propose a QoS-supporting ad hoc network scheme that combines TDMA and IEEE 802.11 DCF. The scheme assigns TDMA time slots for QoS flows by utilizing Network-layer information. The DCF is used to provide contention-based access for best effort flows. All the nodes in the ad hoc network maintain clock synchronization and therefore the identical time period (big frame) structure. The clock synchronization is realized by GPS. Each big frame is divided into two parts, a TDMA period (contention-free access) and a DCF period (contention-based access). Time slots in a TDMA period are reserved according to the demands of QoS flows. In order to improve the channel utilization efficiency, the same time slots are assigned to multiple links if they do not interfere with each other. The time slot assignment procedure uses the routing information. Each node is aware of its one-hop neighbors, two-hop neighbors, and the transmissions in its one-hop region. When a QoS flow is invoked, the caller determines the path for the flow based on the routing information. Each node along the path, i.e. the caller, forwarding nodes and the callee, try to assign time slots by taking account of the neighbor information described above. The authors do not indicate specifically what type of antenna is assumed, yet we believe that the time slot assignment in their algorithm is an interesting avenue to pursue and adapt to MBAs with the goal of exploiting the MPT/MPR capability for delay reduction. 
Bao and Garcia-Luna-Aceves [60] propose the distributed Receiver-Oriented Multiple Access (ROMA) channel access scheduling protocol that uses MBAs. Unlike random access schemes that use on-demand handshakes or signal scanning to resolve communication targets, ROMA determines a number of links for activation in every time slot using only two-hop topology information. It is shown that significant improvements on network throughput and delay can be achieved by exploiting the MPR/MPT capability. The design assumes networks where the two-hop neighbor information or the entire network topology is known beforehand. A neighbor protocol is proposed that uses an allocated random access section to send signals to track neighbor positions for ROMA. The neighbor protocol exchanges neighbor information to synchronize topology information within two hops of each node. In addition, ROMA tries to evenly separate network nodes into transmitters and receivers, so that link activations are maximized in each time slot. The authors do not produce any formal model targeted at minimizing the end-to-end delay. Instead, they use a best-effort, greedy approach. Therefore there is no way of knowing what the minimum E2E delay would be and what routes would produce such a (lower bound) delay in a multi-flow scenario.

In order to enhance the overall network capacity by exploiting the rate diversity with MPR capability, Choi et al. [61] propose a power control algorithm where the receiver instructs the intended transmitters to adjust their transmit power levels to equalize the transmission durations of simultaneously transmitting nodes. Specifically, the nodes which have the longest and shortest transmission duration increase and decrease their transmit power, respectively, in order to use the immediate higher and lower transmission rates. As a result, the proposed algorithm can equalize the transmission durations of all transmitting nodes, increasing the channel utilization. Simulation results show a performance improvement in terms of the aggregate throughput.

If MBAs are to be used at all, having an appropriate MAC protocol is a must. Therefore, we have thus far explored MAC protocols that are designed to work with MBAs. The natural next step is now to review some theoretical analyses on how the mother lode of opportunities that MBAs offer can be further harnessed in order to 
push the performance limits of wireless networks.

\subsection{Performance/Optimization Studies with Multi-Beam Antennas}

This section overviews theoretical analyses and frameworks that are proposed to exploit the benefits offered by MBAs for the purpose of formally optimizing networks' performance metrics, especially the throughput that is so widely explored in the context of MBAs. Wang and Garcia-Luna-Aceves [62] present an approach that takes advantage of the MPR capability of MBAs to reduce the negative effects of multiple access interference and therefore increase the capacity of an ad hoc network. The MPT capability is not considered, nor is the E2E delay performance metric. They formulate an optimization problem under a deterministic model and seek to maximize the aggregate network throughput. They then propose a polynomial-time heuristic algorithm aimed at approximating the optimal solution to the joint routing and channel access problem under MPR. This methodology seems interesting to pursue.

A mathematical framework for analyzing the throughput of an IEEE 802.11 network in the presence of asynchronous MPR is presented in [63]. The authors show the existence of an optimum value for the threshold, which defines the number of ongoing communications that allows a contending node to access the channel. The optimum value represents the best compromise between the throughput improvement, derived from an aggressive approach, and the necessity to avoid excessive collisions.

Another theoretical framework for deriving the throughput of an asynchronous IEEE 802.11 network adopting spatial reuse for enabling Multi-Packet Communication $(\mathrm{MPC}=\mathrm{MPT} / \mathrm{MPR})$ is presented in [64]. The analytical throughput is compared to that obtained from a MAC protocol that reproduces the backoff rules assumed by the analysis, discussing the reliability of the theoretical collision model.

In [65], Li et al. analyze the throughput performance of a wireless network node exploiting an MBA. Using an analytical framework, they derive the Node 
Throughput Gain (NTG) for both MFBA and MCSA. For both MBA structures, the use of MFBA becomes the pessimistic case of MCSA as the normalized beamwidth reaches the maximum value of unity. The upper bound of NTG is achieved in the presence of idealized collision avoidance schemes, whereas the lower bound is reached when no collision avoidance schemes are applied.

Mumey et al. [66] study topology control in MBA-based multi-hop wireless networks with the objective of maximizing the network capacity. Unlike our study, link scheduling in time slots is not explored. Instead, the authors formally define the corresponding optimization problem as the Sector Selection Problem (SSP). They also present a Mixed Integer Linear Programming (MILP) formulation to provide optimal solutions. An effective Linear Programming (LP) rounding based algorithm for SSP is then presented. Simulation results show that the proposed algorithm provides close-to-optimal performance and yields good solutions in terms of both capacity and fairness compared to alternative approaches including a Minimum Spanning Tree (MST) based algorithm and the $k$-nearest neighbors algorithm. Even though the authors do not address the issue of E2E delay, the methodology they use is interesting for our delay reduction study.

In [67], Rokonuzzaman et al. show that MBAs allow the network topology to be adjusted dynamically by adjusting the beamwidth and beam directions to minimize interference and to maximize the number of possible concurrent network communications. This in turn helps to maintain the QoS of the communications.

\subsection{Other Performance/Optimization Studies}

As noted in the previous section, theoretical analyses and frameworks have been proposed to exploit the benefits offered by MBAs mainly for the purpose of formally optimizing networks' throughput. Little attention has been given to E2E delay. In this section, we overview theoretical analyses that either mainly focus on E2E delay or have it constrain other metrics (such as throughput) performance optimization. Even though MBAs are not considered, we find this review to be a worthwhile stop because a) it help us have an overarching perspective on theoretical performance optimization, 
and b) it gives us a sense of how E2E delay optimization can be dealt with. We could later build on those to propose E2E delay optimization in the context of MBAs.

In [68], Bennett and Brown take a linear programming approach to optimally schedule client specific traffic in a mobile hub-and-spoke scenario using a single-beam directional antenna. They use it to maximize client throughput while considering delay constraints as applicable. The beam-switching sequence is optimized using a shortest-path-first approach to mitigate the inter-switch delay and associated protocol specific overhead.

Cheng et al. [69] show that the shortest path does not always lead to the minimum delay. E2E delay being a result of both the number of hops on the path and the interference level along the path, the shortest path leads to the minimum delay only if the shortest path is the least interfered path. The authors propose a linear-programming-based link scheduling scheme that computes time slot assignments in order to minimize the E2E delay without causing conflicting transmissions.

A method for scheduling and synchronizing all transmissions of data in an ad hoc network is proposed in [70]. Data is transmitted on a given path from a given source to a given destination. Time is divided into cycles and in each cycle, each node in the path transmits data belonging to the path during the same time slot reserved for that node and path. Time slots have arbitrary sizes, are reserved via trial and error, and the time slot schedule is iteratively optimized to reduce E2E delay using local coordination rules between nodes. The scheduling method can be used for wireless, wired, acoustic or optical networks. This scheduling method is supported by a formal optimization model.

Thulasiraman and Shen [71] propose a decoupled approach to routing and scheduling optimization for Wireless Relay Mesh Networks (WRMNs) which emphasizes physical interference constraints on capacity and spatial reuse of time slots to maximize throughput using multiple subcarriers. They show that decoupled optimizations improve throughput and minimize time slots by mitigating interference and allowing time slots to be reused in a spatially effective manner while alleviating 
the need for joint interference constraints between routing and scheduling to determine schedulable flows. The idea of minimizing time slots is related to E2E delay minimization to a great extent.

In a nutshell, we have seen in this section that a few authors have proposed E2E delay minimization, solely focusing on omnidirectional and single-beam directional antennas, and therefore never attempting to harness the benefits of MBAs. This is where our work comes in: we want to exploit the benefits of MBAs. Nonetheless, the take away in these authors' work is the general methodology that they employed, consisting of a linear-programming-based link scheduling scheme that computes time slot assignments in order to minimize the E2E delay. Other studies, such as [72-87], unfortunately do not propose E2E delay optimization nor do they consider MBAs, even though most of them, nevertheless, do propose some sort of link scheduling based on a formal linear programming formulation (with other objectives).

\subsection{Throughput Maximization vs. Delay Mini- mization}

The network throughput can be defined $[43,62,88]$ as the average number of packets successfully delivered to all destinations per time slot. Throughout this literature review, most performance improvement or optimization endeavors target maximizing throughput. It is important to emphasize that maximizing the throughput does not necessarily minimize the E2E delay. As we have seen, most of the throughput maximization effort tends to create an optimal TDMA schedule for every node in the network. And, as pointed out by Vergados et al. [73], these schemes result in relatively high E2E delay because they only try to maximize the number of concurrent transmissions within a TDMA slot. Cheng et al. [69] also show that the solution that maximizes network throughput often neglects the delay aspect and leads to poor E2E delay. They make the case that two routing algorithms with different objectives result in different routes. Alvandi et al. [89] claim that when the throughput is maximized, the traffic of a link may approach its capacity, which may result in prohibitively high packet delays in some paths in the network.

As an illustration, in the context of MBAs, let us consider two traffic flows, 
$S 1 \longrightarrow D 1$ and $S 2 \longrightarrow D 2$, in two scenarios depicted in Figure 3.3. The topology is the same in both scenarios; only the path chosen, hence the link scheduling, changes. The delay is measured by the number of time slots it takes for a packet to be delivered from source to destination, on average. And we have already seen that the throughput is measured as the average number of packets received by all destinations per time slot.

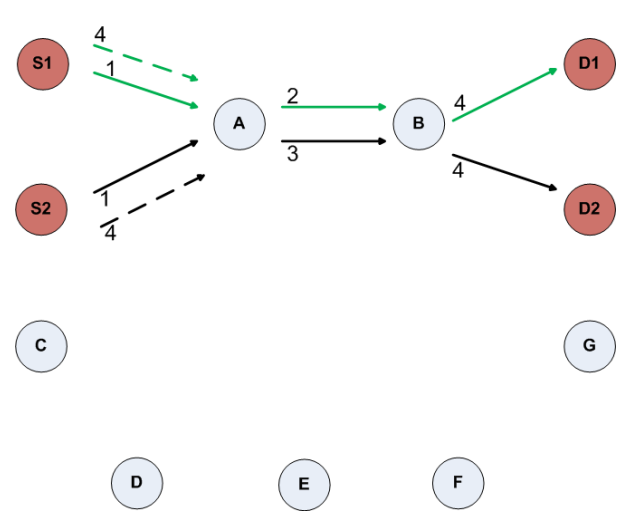

(a) Minimum Delay

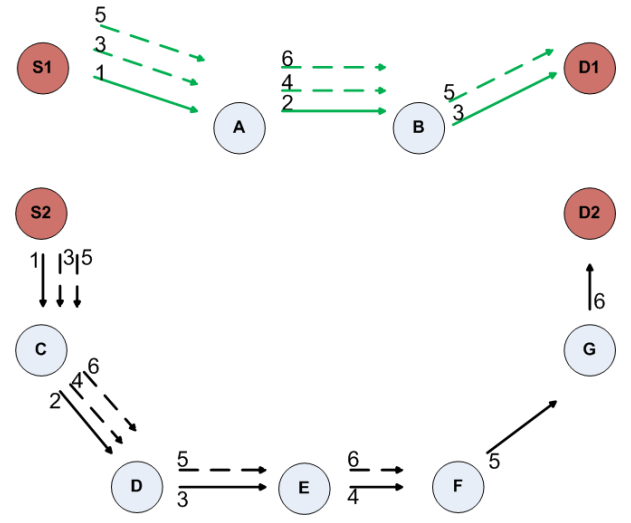

(b) Higher Throughput

Figure 3.3: Delay Minimization vs. Throughput Maximization

We assume that the sources ( $S 1$ and $S 2$ ) have an infinite queue loaded with packets, and they are constantly issuing new packets into the network whenever there is an opportunity (no interference, 2 hops away).

On the one hand, the link scheduling that gives the minimal delay is depicted in Figure 3.3(a), and that minimal average delay is 4 slots per flow. More details on delay minimization with MBAs will follow in Chapter 5. In this case, we have a throughput of $0.66 \overline{6}$ packet per time slot.

The delay with this link scheduling is found by observing that every packet takes 4 time slots to be delivered from source to destination; rightfully giving an average of 4 slots per flow. With this straightforward and simple topology, it is easy to see that this is the minimum average achievable. Other link schedules would give either an equal or a higher average. The throughput is evaluated as follows. It is observed that 
in time slot 4, when the first two packets (those who left the sources $S 1$ and $S 2$ at time slot 1) are reaching their respective destination ( $D 1$ and $D 2)$, two new packets are issued. The new scheduled links are depicted with dashed lines in Figure 3.3(a). The new packets will eventually reach their respective destinations at time slot 7 , and at that point, two other packets will be issued at the sources. And this cycle continues indefinitely. Based on this schedule, in the long run, both destinations receive a new packet every three time slots, resulting in a throughput of $2 / 3=0.66 \overline{6}$.

On the other hand, a different scheduling of the links (with a different path for flow $S 2 \longrightarrow D 2$ ), as depicted in Figure 3.3(b), yields a higher throughput of 1 packet per time slot, but the average delay is now 4.5 slots per flow; which is obviously not the minimal delay achievable. This second link scheduling achieves a higher throughput than the first one; which demonstrates that maximizing the throughput does not necessarily minimize the E2E delay.

The delay with this new scheduling is found by observing that packets in flow 1 are delivered in 3 time slots and packets in flow 2 are delivered in 6 time slots; giving an average of 4.5 slots per flow. The throughput is found by noting that in time slot 3, when the first packet that left source $S 1$ at time slot 1 is reaching its destination $(D 1)$, a new packet is issued at $S 1$. Plus, the first packet that left source $S 2$ at time slot 1 is reaching intermediate node $E$, and at that point (time slot 3), a new packet is issued at $S 2$. The new scheduled links are depicted with dashed lines in Figure 3.3(b). The first packet issued by $S 2$ will eventually reach its destination $D 2$ at time slot 6 , and at that point, the second packet issued by $S 1$ will have been received one time slot earlier (slot 5), the third packet issued by $S 1$ at time slot 4 will be reaching intermediate node $B$. The issuance of packets and scheduling of links for both flows over the first 6 time slots are depicted in Figure 3.3(b). This cycle continues indefinitely. From time slot 5 onwards, a new packet is always received at one of the two destinations at each slot. In the long run, a new packet is received at either destination (not both) at every time slot, resulting in a throughput of 1 .

From underlining claims in the literature to an example, the foregoing discussion shows that throughput maximization is not equivalent to E2E delay minimization. This further points out the fact that E2E delay minimization with MBAs is still to 
be explored.

Moreover, explicitly modeling packet latency provides us with the ability to tackle other QoS issues such as delay-bounding, basically keeping the delay of each individual flow within certain bounds. For instance, with two flows, instead of having one short delay and one long delay that, together, give the minimum average delay, we can choose a solution/scheduling that gives a higher average delay but with individual delays of the flows kept within some bounds. This could be interesting when all nodes run delay-sensitive applications that cannot afford long delays even if the overall average is the lowest possible. As an illustration of this, let us consider the 12-node topology depicted in Figure 3.4 with five traffic flows as follows: $1 \longrightarrow 15$, $3 \longrightarrow 13,2 \longrightarrow 14,16 \longrightarrow 1$, and $13 \longrightarrow 4$. Let us assume that the applications have a hard delay restriction of 5 time slots at most.

On the one hand, the link scheduling that gives the minimum average delay is depicted in Figure 3.4(a) (and Table 3.1), and that minimum average delay is 4.8 slots per flow. In this case, we can see that one flow has a very short delay of 3 slots, and one flow has a prohibitive delay of 6 slots that violates the delay requirements of the running applications. On the other hand, a different scheduling of links as depicted in Figure 3.4(b) (and Table 3.2) yields a higher average delay of 5 slots per flow, with all the flows meeting the 5-slot delay requirement of the applications. This is evidence that not only does having a delay optimization model allow us to minimize the average E2E delay, but also it gives us the means to meet other E2E delay-related objectives (such as enforcing latency bounds) that might be dictated by the targeted applications.

\subsection{Summary}

In this chapter, we have reviewed the use of MBAs and link scheduling for performance improvement in wireless networks. The work on MBAs in the literature focuses mostly on improving network capacity and throughput in infrastructure and 
(13)
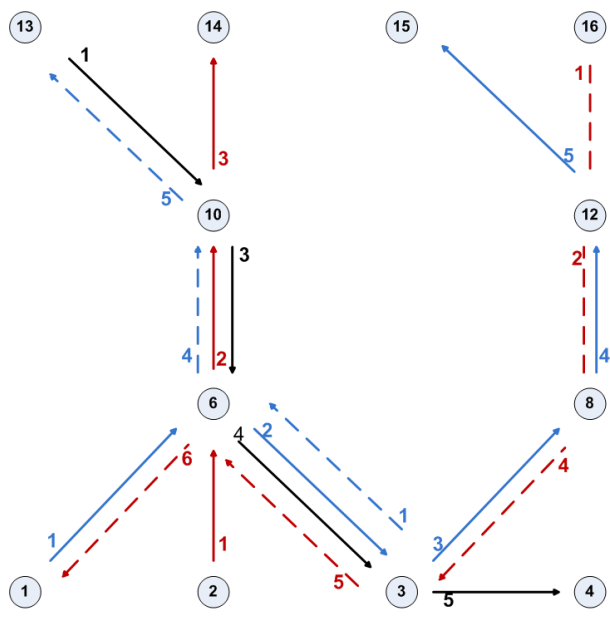

Flow 1: 1->15

Flow 2: 3->13 $\rightarrow \rightarrow$

Flow 3: 2->14 $\longrightarrow$

(a) Minimum Average Delay
(15)

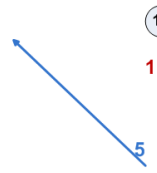

(12)

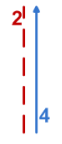

(8)

Flow 4: 16->1 $\rightarrow$

Flow 5: 13 $>4 \longrightarrow$

Figure 3.4: Minimum Average Delay vs. Higher but Bounded Delays

Table 3.1: Minimum Average Delay Link Scheduling

\begin{tabular}{lllllll}
\hline Traffic flow & slot 1 & slot 2 & slot 3 & slot 4 & slot 5 & slot 6 \\
\hline $1 \longrightarrow 15$ & $(1,6)$ & $(6,3)$ & $(3,8)$ & $(8,12)$ & $(12,15)$ & \\
$3 \longrightarrow 13$ & $(3,6)$ & & & $(6,10)$ & $(10,13)$ & \\
$2 \longrightarrow 14$ & $(2,6)$ & $(6,10)$ & $(10,14)$ & & & \\
$16 \longrightarrow 1$ & $(16,12)$ & $(12,8)$ & & $(8,3)$ & $(3,6)$ & $(6,1)$ \\
$13 \longrightarrow 4$ & $(13,10)$ & & $(10,6)$ & $(6,3)$ & $(3,4)$ & \\
\hline
\end{tabular}

Table 3.2: Delay Within Bounds

\begin{tabular}{llllll}
\hline Traffic flow & slot 1 & slot 2 & slot 3 & slot 4 & slot 5 \\
\hline \hline $1 \longrightarrow 15$ & $(1,6)$ & & $(6,10)$ & & $(10,15)$ \\
$3 \longrightarrow 13$ & $(3,6)$ & $(6,10)$ & & & $(10,13)$ \\
$2 \longrightarrow 14$ & $(2,6)$ & & & $(6,10)$ & $(10,14)$ \\
$16 \longrightarrow 1$ & $(16,12)$ & $(12,8)$ & $(8,3)$ & $(3,2)$ & $(2,1)$ \\
$13 \longrightarrow 4$ & $(13,14)$ & $(14,15)$ & $(15,12)$ & $(12,8)$ & $(8,4)$ \\
\hline
\end{tabular}


infrastructureless (ad hoc) networks through medium access protocols and routing algorithms. On the rare occasions where improving the delay is attempted with MBAs for ad hoc networks, the authors do not produce any formal analysis targeted at minimization. A best-effort approach is used instead.

A formal analysis is used mostly for omnidirectional or single-beam directional antennas. The popular approach for finding optimal link scheduling consists of: a) developing a MILP formulation (formal mathematical model) of the problem to provide optimal solutions; then b) developing a polynomial-time heuristic algorithm.

As we know, when MBAs are used, a change of paradigm needs to take place. For instance, transmissions to the same destination can be assigned at the same time slot; something that is impossible with omnidirectional or single-beam directional antennas. However, with MBAs the limitations are that: a) the number of transmissions must be less than or equal to the number of beams of the destination's antenna, and b) all the transmissions must reach the destination at different beams. Transmissions that reach to the same destination at the same beam should be assigned to different time slots. Secondly, a node can perform multiple packet transmissions at the same time; something that is also impossible with omnidirectional or single-beam directional antennas. However, the node must use one beam for each transmission. Therefore, the number of concurrent transmissions is limited by the number of available beams.

The focus on designing a MAC protocol for MBA is justified by the fact that traditional MAC protocols, based on the IEEE 802.11 standard, are designed assuming omnidirectional and (recently) single-beam directional antennas, and therefore cannot take advantage of the unique capabilities of MBAs as they do not facilitate concurrent transmissions or receptions by a node. Some of the proposed MAC protocols for MBAs are TDMA-based; time slots are dynamically allocated for each beam of every node with or without a central coordinator. A lot of MAC protocols that are proposed for MBAs are designed for infrastructure/centralized (one-hop) networks such as cellular networks and WLANs where only the BS or the AP is equipped with MBAs. Allowing as many parallel uplink data transmissions as possible helps improve the throughput. 
This review has shown that exploiting the full potential of MBAs (MPT and MPR) to minimize (versus best-effort reduction) the E2E delay in ad hoc networks has not been explored thus far. That is what we are proposing to do in this work: take full advantage of the MPT/MPR capability of MBAs to optimally schedule links in time slots in order to reduce the E2E delay of an ad hoc network to its conceptually achievable minimum. We are going to adopt the popular methodology of an MILP formulation of the optimization problem and subsequent development of an algorithm. In our case, the algorithm to develop is a routing protocol that will exploit the full potential of MBAs to minimize the E2E delay in ad hoc networks. In this chapter, we have underlined that throughput maximization (widely explored in the literature) is not equivalent to E2E delay minimization. Furthermore, we have pointed out that having a delay optimization model allows us to also meet other E2E delay-related objectives (such as keeping the individual E2E delay of all flows below a given bound) that might be dictated by the targeted MANET applications.

Before proposing any delay minimization routing protocol, we will first need to design and implement a MAC protocol that takes advantage of MBAs. We will adopt a random-access and distributed approach. Like in the case of SBAs, many MAC protocols specifically designed for MBAs in ad hoc networks have been proposed in the literature. But, with no MBA MAC protocol at our disposal (available in OPNET), we design and implement a generic one that is representative of features and mechanisms found in the literature. In the design, we even introduce some unique/novel features discussed in the next chapter. 


\section{Chapter 4}

\section{MBA-DbMAC: A Random-Access MAC Protocol for MBAs}

\subsection{Introduction}

For the MAC protocol running underneath our routing algorithm, we are not going to use a TDMA MAC, but rather a CSMA/CA MAC. This is because enforcing time synchronization between nodes in MANETs in general, and in FANETs in particular, can be very challenging. As noted by Luo et al. in [90], current TDMA-based MAC protocols cannot provide the rapidness and agility to deal with the rapid mobility and varying densities of vehicles in Vehicular Ad hoc NETworks (VANETs) ${ }^{1}$. If this is true for VANETs, it then becomes even more true for FANETs where the mobility is higher and topology changes are more frequent and less predictable since the UAVs are not constrained by roads and highways like in VANETs. Among others, Abolhasan et al. [91] also point out the fact that CSMA/CA is the practical MAC protocol for WDNs, because it does not require time synchronization and there is no centralized coordination. CSMA/CA has been extensively implemented in WDNs.

Note that, even though the arrivals/departures of data packets do not occur at the exact same time, with the CSMA/CA MAC, a Forwarding Node (FN) might be waiting for a CTS or ACK from one flow while another flow sends in its data packet, etc. Therefore, the separation of flows across the beams (bridge avoidance + star formation) of that FN still makes a considerable difference inasmuch as multiple reception tasks (likewise multiple transmissions tasks) can occur concurrently,

\footnotetext{
${ }^{1}$ Another type of MANETs
} 
reducing E2E delay along the path. It follows that, in the MBA MAC protocol, we need to unequivocally define the duration of the receive mode and that of the transmission mode, bearing in mind that all beams are always in the same mode (see the transmission/reception rule in Chapter 3). There needs to be some agreement between the beams such that the switches to Receive mode or Transmit mode are performed in a way that accommodates both an RTS/CTS exchange involving one beam and a Data/ACK exchange involving another beam.

\subsection{Bottom Line}

With the level of abstraction in literature papers that propose MBA MAC protocols, it would be a daunting task to try and implement them solely based on the provided descriptions. For this reason, we propose a MAC protocol of our own for MBA environments. The goal of this protocol is to implement/support the most significant MBA capabilities/features.

In addition to the typical problems inherent to the design of MAC protocols in wireless networks, MBAs introduce new challenges. As already noted, one of these challenges is synchronization. To harness the Concurrent Packet Transmission (CPT) and Concurrent Packet Reception (CPR) capabilities of MBAs, there needs to be some synchronization to ensure that as many transmissions as possible happen while the concerned node is in transmission $(\mathrm{Tx})$ mode before switching to reception $(\mathrm{Rx})$ mode, and vice-versa. Another challenge is Head of Line (HOL) blocking. It needs to be ensured that a packet whose next hop lies in a given beam that is free (medium cleared) is not blocked in a queue behind another packet whose next hop lies in another beam that is waiting for the medium to clear out in that direction. The above challenges and a few more are addressed in the next section. 


\subsection{MBA Challenges}

\subsubsection{Synchronization for CPT and CPR}

Transmitting nodes should start their transmission concurrently so that the common receiver node can simultaneously activate multiple beams pointing toward them. In Figure 4.1, assume that nodes $A, B$, and $C$ need to send data to nodes $E, F$, and $G$, respectively, via node $D$. In the absence of any localized synchronization, the possibility that any two or all three of them will start transmission at the same time using a random access protocol is rare. This is due to the fact that, before initiating transmission, each node waits for a random duration after sensing the channel idle for DIFS duration. Therefore, node $D$ could start receiving a packet from node $A$. Before that reception is over, nodes $B$ and $C$ could begin their transmissions to $D$ as well. Any of these transmitting nodes might have many packets to transmit and start transmitting those packets before the ongoing transmissions are over. This chain of events would trigger what is known as transmission starvation on node $D$ which would be locked in successive receptions. To avoid such starvation, [2] proposes that CPR and CPT occur in succession at the bottleneck node (node that is common in two or more routes). To us, even without enforcing that all transmitters start their transmissions at the same time (as is the case in [2]), this scheme would translate into only accepting one packet per transmitter per cycle. That way, after having received packets from all sources, node $D$ would switch to CPT and transmit them all together to their respective destinations, as shown in Figure 4.1. We shall present our full strategy on this CPR/CPT issue in a subsequent section.

\subsubsection{Head-of-Line Blocking}

A packet at the head of the data queue may block other packets behind it indefinitely if its intended outbound beam is busy. This phenomenon is known as Head of Line blocking [92]. This can be prevented by having a dedicated data queue for each beam as is the case in HMAC [2]. 


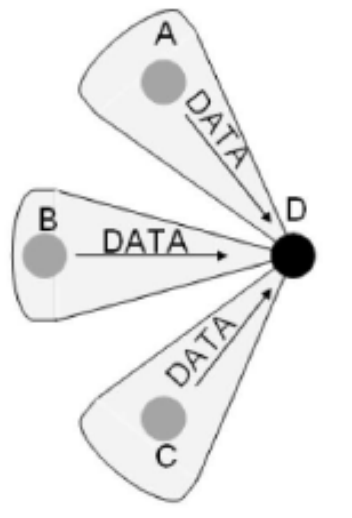

(a) CPR
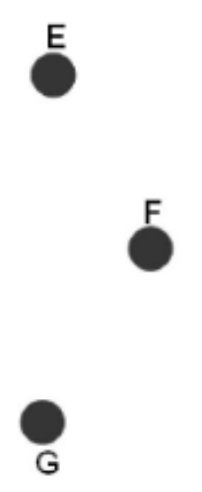

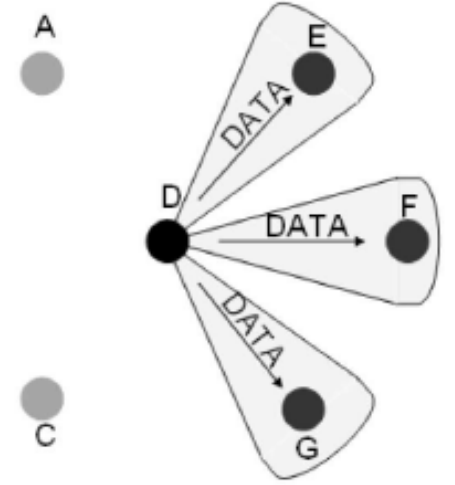

(b) $\mathrm{CPT}$

Figure 4.1: CPR and CPT [2] (C)2008 IEEE

\subsubsection{Deafness}

In [2], the authors point out deafness as a problem, invoking beamforming. However, beamforming is not applicable to MBAs. It is only a reality for single-beam directional antennas (SBAs). Therefore, we believe that deafness is a non-issue in the context of MBAs. As already discussed in Chapter 2, a source node experiences the deafness problem when it fails to communicate with its intended destination node that is

pointing towards a different direction for transmission or reception. In fact, we should only consider the case when the intended destination is pointing to another direction for reception. That is because in the case of transmission, even if the destination was pointing to the source, there would be a mode mismatch and the packet from the source would not be received, as the destination cannot be transmitting and receiving at the same time. Now, in the case of MBAs, if the destination node is receiving from a different direction for reception, the beam that is pointing to the source is also able to receive, since the reception mode applies to the entire node (i.e. all beams) and multiple beams can receive concurrently. In SBAs, only one beam can be active at a time.

\subsubsection{Hidden-Terminal}

A given scenario experiences the hidden-terminal problem when transmissions from two nodes which cannot hear each other collide at a third node. In the context of directional antennas, all nodes that are located within the destination node's coverage 
area of interest (covered by a specific beam) and are away from the source node's coverage area of interest are hidden terminals. The shaded area $A_{h}$ in Figure 4.2 indicates the area in which hidden terminals may exist, from the perspective of node $S$. A node located at any other area where it cannot hear $S$ is not a hidden terminal because, even if it points toward $D$, its signal either will reach $D$ from a different beam (therefore allowed to be processed thanks to CPR capability) or it will be undetectable (because too far away) by $D$. Unfortunately, as pointed out in [3], the standard RTS/CTS mechanism fails to completely solve the hidden-terminal problem, as nodes in $A_{h}$ may initiate transmissions to $D$ during the time the source node $S$ transmits the RTS to $D$. This problem has not been solved in current literature using a single-channel and single-radio interface [93].

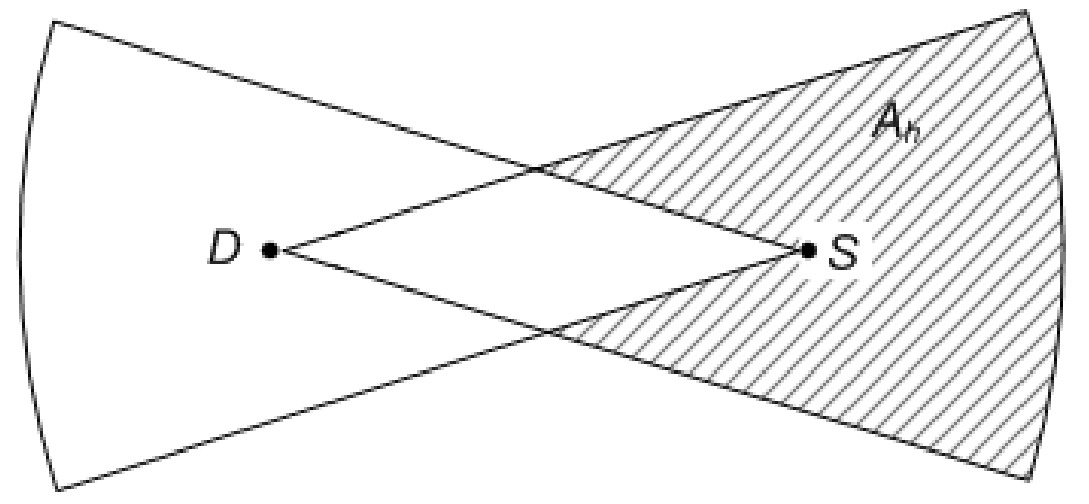

Figure 4.2: Hidden-Terminal Problem [3] (c)2012 IEEE

\subsubsection{Exposed-Terminal}

A node experiences the exposed-terminal problem if it assumes a busy medium and defers its transmission even though it could be transmitting without impeding ongoing transmissions by other nodes. This is a problem mostly for omnidirectional antennas. As pointed out in [4], and as shown in Figure 4.3, assume that node $A$ wants to transmit to node $B$. Node $A$ sends an RTS and waits for $B$ to send a CTS. Suppose node $D$, located in area $Y(x)$, wants to transmit data to node $C$ located in area $X(x)$, and $D$ transmits an RTS to $C$ just before $A$ sends the RTS to $B$. After receiving the RTS from $D, C$ transmits a CTS. This CTS is heard by $B$ upon which $B$ will enter a backoff period preventing $B$ from sending the CTS to $A$. Therefore, any transmission from a node within the area $Y(x)$ to a node within $X(x)$ 
will prevent $A$ from transmitting data to $B$, although simultaneous transmissions from area $Y(x)$ to $X(x)$ would not have interfered with transmission from $A$ to $B$. The terminals in the region $Y(x)$ are the exposed terminals for the node pair $A / B$.

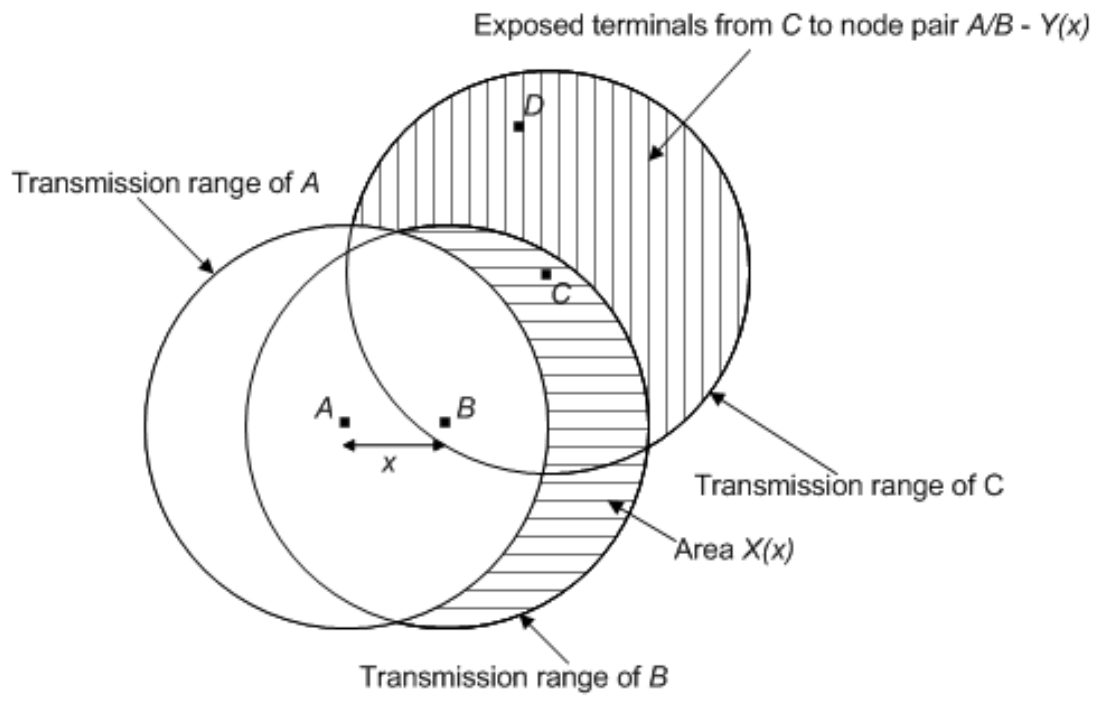

Figure 4.3: Exposed-Terminal Problem [4]

In the context of directional antennas, the exposed-terminal problem can easily be solved by taking advantage of directionality. In effect, the exposed-terminal problem happens when a node receives an unintended RTS/CTS and blocks its antenna for the duration of the announced transmission, preventing the node from participating or initiating concurrent transmissions that would not impede the ongoing one. Simply using directional (per sector) NAV timers narrows the scope of this issue, since only the sectors receiving the unintended RTS/CTS will be blocked. Notice that, even with directional antennas, there is still the possibility of an exposed-terminal if the parallel transmission falls within the blocked beam (See Figure 4.4 where the transmission between $C$ and $D$ is prevented by the transmission between $A$ and $B$ that has blocked $C$ 's beam that points to $D$ ); but we assume that this is infrequent enough to not justify a separate mechanism to deal with it. 


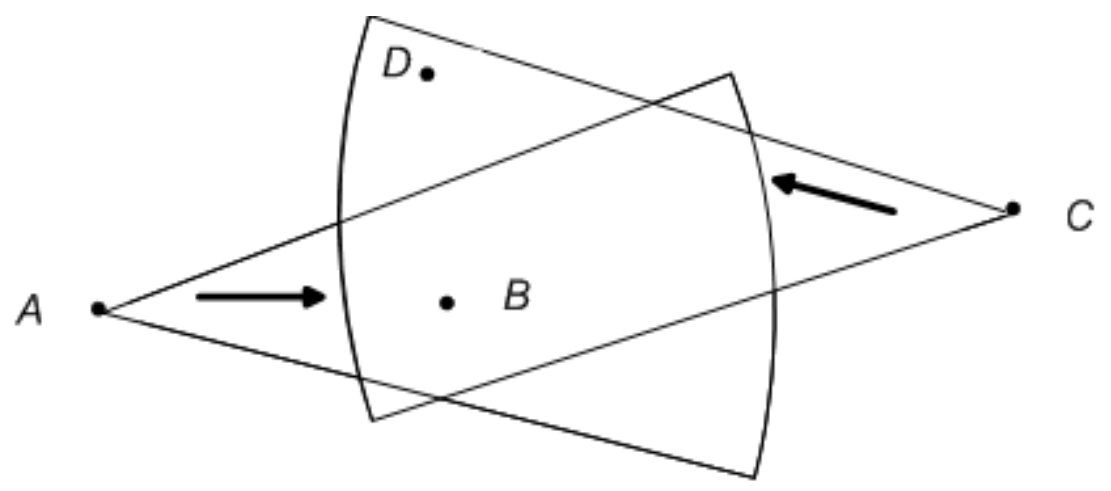

Figure 4.4: Exposed-Terminal Problem with Directional Antennas

\subsubsection{Random Backoff: Beam-Based or Node-Based}

In MBA, each node has several beams, so the problem is how to control the random backoff for each beam after DIFS during transmission or retransmission attempts. One solution is to maintain a separate contention window (CW) for each beam, referred to as beam-based backoff. The other solution is to have a common CW for all beams that increases or decreases depending on the collisions or successful transmissions in the transmitting beams. This scheme is referred to as node-based backoff, where a node can transmit in multiple beams simultaneously. Now all the beams wait for the same random duration after DIFS. This is the approach adopted in HMAC and MMAC-NB [94], where the authors claim that it is conducive to high shares of CPT. This approach implies that if the medium in one beam becomes active, the backoff is suspended in all beams; which, we believe, would not be efficient. Therefore, we do not intend to pursue this approach. Instead, the beam-based backoff approach seems more appropriate.

\subsection{Antenna Model}

Similar to the approach proposed in [2], we focus on gains from spatial reuse exclusively and not from range extension of directional beams. Therefore, the range of each beam is constant and is kept equivalent to the omnidirectional range. The antenna is assumed to have a power control mechanism that feeds each beam with a power of $P_{T O T A L} / M ; M$ being the total number of beams.

Since the transmission range is kept constant, the implication is that the amount 
of power used will vary depending on the number of beams that are activated for a given transmission cycle. Broadcasting and multicasting (using multiple beams to send multiple different unicast packets) on all beams will consume the most power while unicasting will consume the least power. In the end, gains in energy efficiency are expected inasmuch as it is anticipated that we will not use all the beams at every single transmission cycle.

The beam shape is assumed to be a disk slice (in 2D). Sidelobes' interferences are not considered for simplicity and simulation tractability. Carrier sensing is performed directionally, that is, before transmission, the medium is sensed only in the desired beam(s). In idle mode, the receiver listens on all its beams (omni mode). A collision occurs only if a node receives interfering energy on the same beam in which it is actively receiving a packet. We choose to work with a switched-beam antenna model for the reasons discussed in Section 3.2. A switched-beam antenna requires only activating one of the predefined beams that concentrates in the direction of the user. An adaptive beam antenna requires complex beamforming algorithms to point in the direction of the user. Thus, adaptive beam antennas are more complex to design and are not generally considered for commercial wireless networks [2]. Hence, in the rest of our work, an MBA refers to a switched-beam antenna capable of switching multiple beams simultaneously. A node can either transmit or receive data, but not both, on multiple beams at the same time.

\subsection{A Hybrid MAC Solution}

MMAC-NB [94], ESIF [95], and HMAC [2] were all proposed by the same authors. In this section, we summarize only HMAC because it is considered an upgrade of the former two. In effect, although ESIF and HMAC deliver comparable performance, HMAC fares better; owing to its simpler design, reduced cross-layer dependence, and backward compatibility with IEEE 802.11-DCF-based protocols. The reactive mechanism for handling deafness and p-persistent CSMA employed by ESIF requires modifications in the Network layer to store the count of potential transmitters in every beam and message piggybacking among the neighboring nodes, which increases the overhead and complexity of the protocol. On the other hand, MMAC-NB has a poorer Concurrent Packet Reception capability, which leads to the underutilization 
of multiple beam antennas at the bottleneck (star) nodes.

As discussed in Section 4.3.3, we do dispute the existence of the deafness problem when MBAs are used. In effect, the authors in [2] claim deafness as follows. In Figure 4.5, it is assumed that nodes $A$ and $B$ are engaged in communication, and control packets are transmitted directionally. Hence, nodes $X$ and $Y$ are oblivious to the ongoing communication between $A$ and $B$. The claim is that they continue transmitting RTS messages to node $A$ who is deaf to their messages and hence does not send back any CTS messages to nodes $X$ and $Y$ (which, consequently, go into backoff mode). In fact, in an MBA scenario, $A$ cannot be deaf to the transmissions from $X$ and $Y$. It will simply receive those transmissions on different beams at the same time as it receives the transmission from $B$.

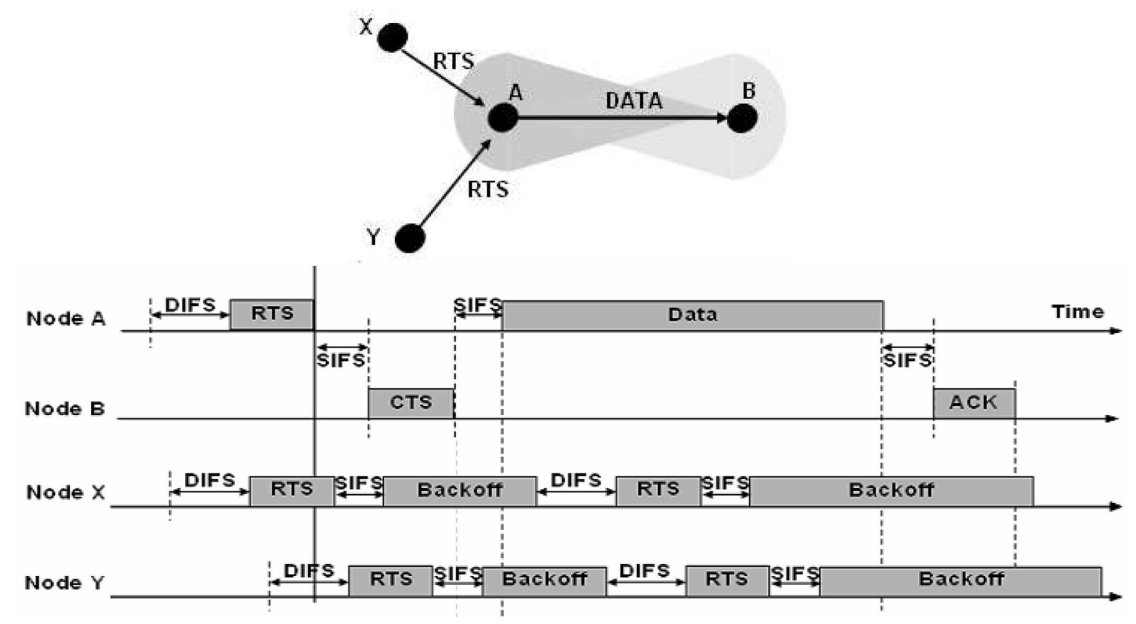

Figure 4.5: Illustration of Deafness as per [2] (C)2008 IEEE

HMAC is a cross-layer protocol that uses information from both the Network and the Physical layers for its operation. Similar to MMAC-NB and ESIF, HMAC uses a separate queue for each beam to avoid HOL blocking. It also uses a scheduling (SCH) control packet, which is sent in all desired beams other than the ones being negotiated via RTS/CTS. The purpose of these additional SCH packets is to further mitigate the deafness problem by letting potential transmitters know that the current node is pointing somewhere else. The novel features of HMAC include: its channel access mechanism, algorithms for mitigating deafness and contention resolution, 
as well as jump backoff and role priority switching mechanisms for enhancing throughput. HMAC is also backward compatible with IEEE 802.11 DCF.

To minimize queuing delays in the network by facilitating successive cycles of CPR and CPT, a mechanism similar to hot-potato routing [96] is installed at every node. Thus, depending on the packets in its buffer, a node switches between transmitter and receiver modes. As long as a data packet exists in the queue, the node gives priority to the transmission mode; otherwise, the reception mode supersedes. Moreover, depending on the available neighbor and beam schedules, a node can determine whether it can actually initiate data transmission. If not, the reception mode gets priority.

\subsection{Our Solution: The MBA-DbMAC Protocol}

The IEEE 802.11b DCF MAC protocol (omnidirectional) is contention-based. It employs a CSMA/CA mechanism by means of the DCF (Distributed Coordination Function). In Chapter 2, we adapted this standard MAC protocol to work with singlebeamed directional antennas. We called the new adapted MAC protocol the IEEE 802.11b-based Directional MAC (or DbMAC) protocol. DbMAC works mostly like the standard IEEE 802.11b DCF MAC protocol but on a per-antenna-sector basis. At this juncture, we are going to adapt DbMAC to work with MBAs. We call this new protocol the MBA-DbMAC protocol. This new protocol is going to adopt, to a certain extent, some of the approaches of HMAC (thus of MMAC-NB and ESIF as well) to solve some of the MAC protocol challenges that come with the introduction of MBAs as discussed earlier. In the spirit of DbMAC versus existing single-beam directional MAC protocols, MBA-DbMAC combines and/or slightly modifies the common/key features of existing MBA MAC protocols without much of a claim of being a superior protocol. Nevertheless, we do propose a novel decoupled broadcasting scheme whose goal is to give all the beams a chance to transmit when some are not ready during the first broadcasting attempt. The details of MBA-DbMAC are summarized in the next few paragraphs. 


\subsubsection{Design}

For the design of the MBA-DbMAC protocol, we adopt a two-tier processing approach. In effect, we split the MAC layer into two artificial sub-layers: the controller sub-layer and the sector sub-layer. Figure 4.6 depicts the contrast between this approach and the traditional single-tier approach. In practice, in the development environment that we use (OPNET), these two sub-layers are materialized with processes: each node has one controller (parent) process that spawns $N$ identical sector (child) processes at run-time; $N$ being the number of antenna sectors. Traditional MAC-layer mechanisms are applied at the sector sub-layer. The controller sub-layer manages: a) the neighbor table, b) the assignment of a high-layer packet to the appropriate sector, and c) the switch between the different operation modes (Tx mode, Idle mode, and Rx mode). The operation mode applies to the whole node. For instance, if a given sector is permitted to transmit at a given time, it is the job of the controller process to instruct all the other sectors via their respective processes to now switch to Tx mode, regardless of whether or not there are packets to be transmitted by these other sectors. Our two-tier design approach is an elegant way of enforcing mode switches in the node while still leaving the full autonomy of medium access to sectors in their respective direction of competence.

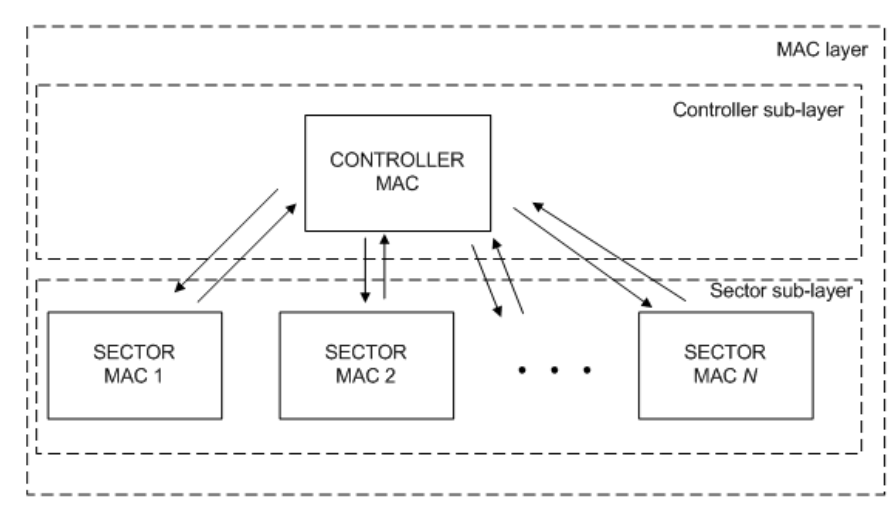

(a) Two-Tier MAC Approach

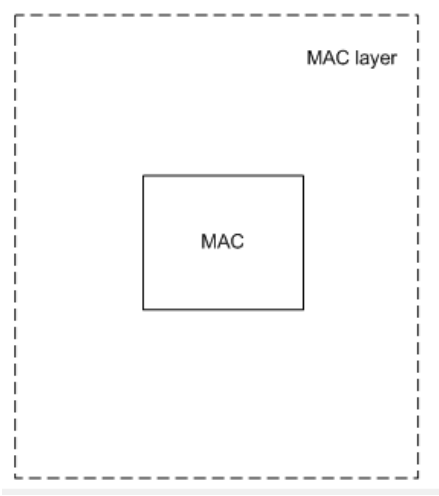

(b) Traditional Single-Tier MAC Approach

Figure 4.6: Two-tier MAC Approach Vs Traditional Single-tier MAC Approach 


\subsubsection{Ensuring CPT and CPR}

Unlike DbMAC, we now allow multiple packets to be received or transmitted at the same time. This also means ensuring the processing of such packets by the node, as long as this is happening on different beams. The reception of two or more packets on the same beam at the same time is considered a collision. Likewise, the transmission of two or more packets on the same beam at the same time is forbidden.

We do not enforce that all transmissions on all beams of interest start at the exact same time. However, once a transmission starts on a given beam, the node is now in transmission mode (no reception can occur in any beam at this point). Any other beam that is scheduled to start a transmission while the first is still going on can start its transmission, provided a certain condition that we discuss later in this paragraph. The transmission mode ends when all beams have ended their respective transmissions. However, to ensure fairness and avoid reception starvation, we limit each beam to only transmit one packet per cycle. Backoff and IFS decisions are made on each beam independently of the others. This means we opt for a beam-based backoff approach, unlike HMAC and ESIF. Once the transmission mode ends, the node goes to Idle mode, or goes to reception mode if a signal is sensed right at that point in time. The node can also start a new transmission cycle if there is no signal sensed and there are packets ready to be sent instead; with the medium sensed idle long enough (has been clear for an IFS duration). We call Critical Chain Transmission (CCT) a situation where all antenna sectors/beams start transmitting one right before the end of the transmission at another sector. All the sectors are therefore stuck in transmission mode until the last sector of the chain has finished, even though the first transmitting sector has finished its transmission a while ago. CCT might result in spending a disproportionate amount of time in one transmission mode that quickly becomes useless to most sectors. This will then incur some MAC delays. To avoid CCT, we set up the transmission mode such that any sector can only start its transmission within a certain time window after the first sector has started its transmission. Past this time window, no transmission is allowed to start until the next transmission cycle (after the current transmission mode has completed). We set the time window to be half the anticipated transmission time of the first transmitting sector. 
We ensure CPR using the same philosophy as with CPT. A node simply stays in $\mathrm{Rx}$ mode until all concerned beams have completed their respective receptions. The rule of one packet per beam per cycle also applies to avoid transmission starvation if there are too many packets coming in. Once a given beam has finished receiving one packet, it deactivates itself from further reception for the remainder of that Rx cycle. All packets (unicast and broadcast) arriving during this deactivation period will be lost. The senders of those unicast packets will have to retry sending if applicable. The $\mathrm{Rx}$ mode ends when all beams that have sensed a packet before the end of the first reception finish their respective receptions. The node then switches to Tx mode if there is a packet in any of the queues and the medium is ready to be used in the concerned direction. If there is no packet ready to be sent (medium also ready/available), the node goes into Idle mode or a new $\mathrm{Rx}$ mode if an incoming signal is sensed right at that time. From Idle mode, a node can go into Tx or Rx mode, depending on which one occurs first: a packet ready to be transmitted and the medium free, or an incoming packet sensed at the Physical layer. We choose to not adopt the approach to synchronize potential transmitters, because that would be a difficult and overhead-inducing undertaking. Similar to the CCT, the Critical Chain Reception is a situation where antenna sectors/beams start receiving a packet right before the end of the reception at another sector. We similarly adopt a time window policy to avoid CCR. In effect, we set up the reception mode such that any sector can start reception only within a certain time window after the first sector has started its reception. Past this time window, no reception is allowed to start until the next reception mode in the next cycle (after the current reception mode has completed). We set the time window to be half the anticipated reception time of the first receiving sector. In this case, the anticipated reception time is approximated to the time it would take the current node to transmit a packet the size of the RTS-threshold (the threshold for performing RTS/CTS frame exchange preceding the transmission of the data frame.) to the neighbor.

\subsubsection{Switch from $\mathrm{Tx}$ to $\mathrm{Rx}$ mode}

As already mentioned, we set $\mathrm{Rx}$ and $\mathrm{Tx}$ modes to alternate, with one packet reception/transmission allowed per sector per cycle. The default mode is the Idle mode, where a node is neither transmitting nor receiving on any of its sectors. The switch to $\mathrm{Tx} / \mathrm{Rx}$ mode is interrupt-driven. In the case of the switching-on of the 
Tx mode, a node will know when a packet is passed down the protocol stack or has been received and now has to be forwarded. The node checks the medium on the beam of interest. When the medium is ready (after SIFS/DIFS and backoff waiting if applicable) for transmission, a switch to Tx mode occurs, provided that the node is in Idle mode. The node stays in this mode until the transmission is complete and there is no other transmission in progress on any other beam. It then switches back to Idle mode. From the Idle mode, the node can now switch to Rx mode when an incoming packet is sensed by any sector. If no incoming packet signal is sensed at the Physical layer and there is a packet to be transmitted and the medium is free, then a switch to Tx mode occurs. As already mentioned, each node features a controller process that instructs the switch to $\mathrm{Rx}$, $\mathrm{Tx}$, or Idle mode to all the sectors. The state diagram of the controller is depicted in Figure 4.7 and the state diagram of the sector/beam is shown in Figure 4.8. For instance, while in Idle mode, if an incoming packet is sensed on a sector, that sector starts the reception and immediately reports it to the controller process. The controller process then instructs all the other sectors to switch to Rx mode. At this point, only receptions are allowed. Similarly, while in Idle mode, if the medium becomes available in a certain direction where a packet is waiting to be sent, the concerned sector starts the transmission and informs the controller. The controller then instructs all the other sectors to go into Tx mode. These other sectors can now start their own transmissions, if any, as long as they start within a certain time window as discussed earlier.

Once in Tx mode, all the sectors involved have different transmission end times. The node ends its Tx mode when the latest transmission end time occurs. The same applies to the Rx mode and the different reception end times.

In the special case of a Backoff period ending (for a transmission to start) at the same time as a packet reception starts on a given sector, preference is given to the transmission, and the packet reception is aborted. 


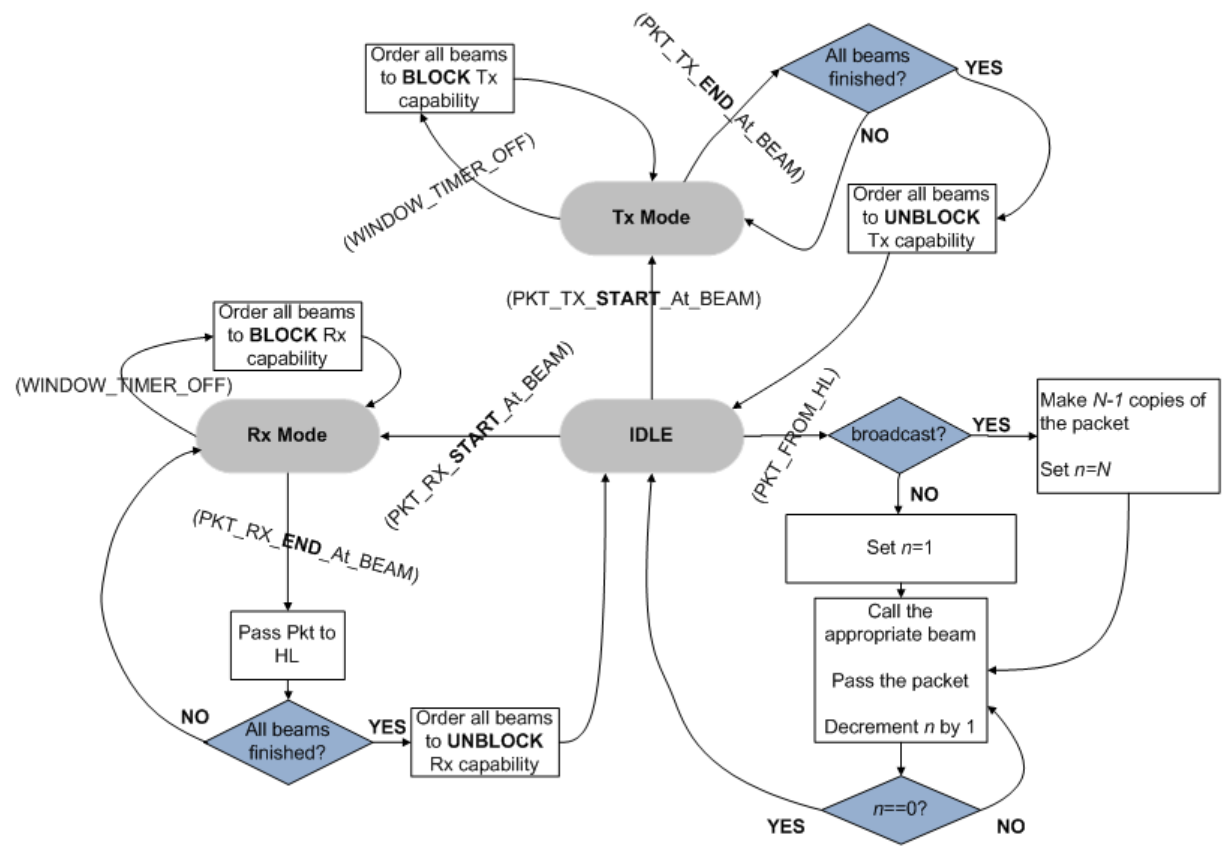

Figure 4.7: Controller/Parent Diagram

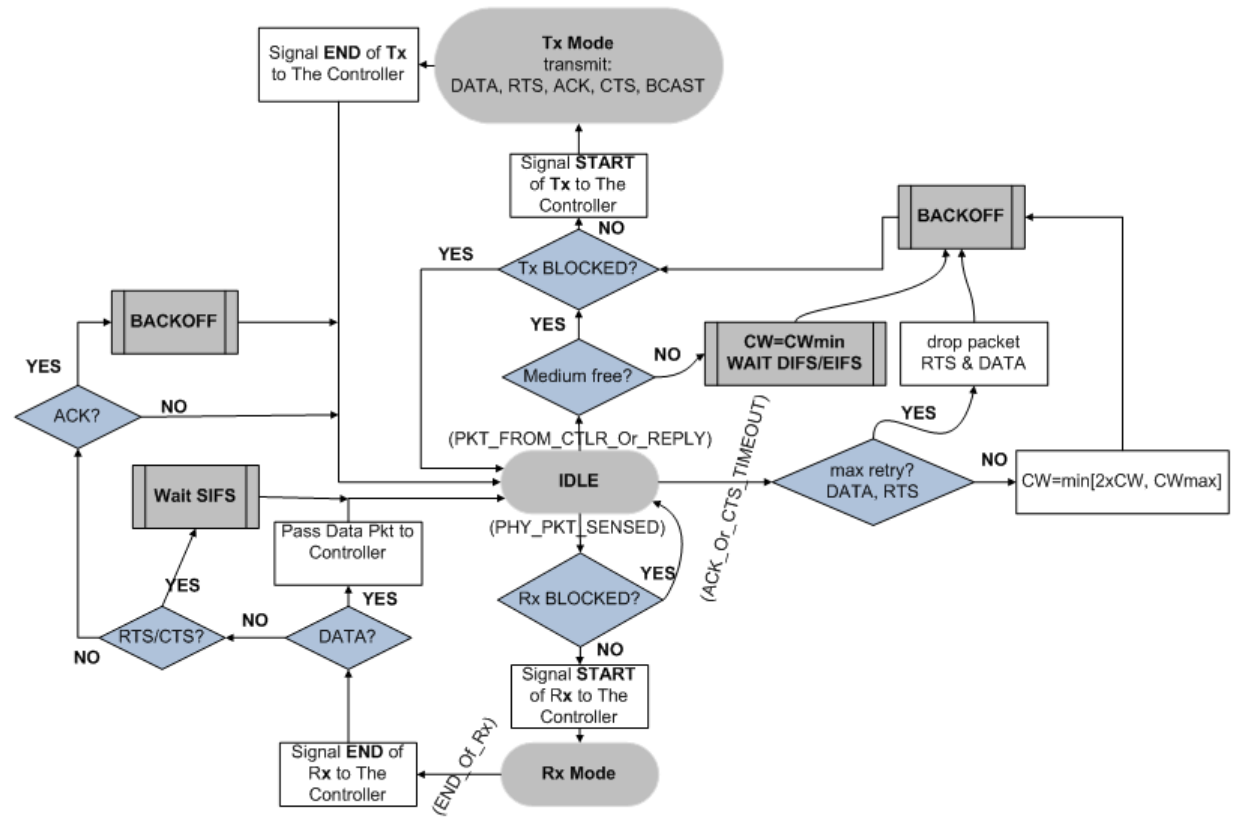

Figure 4.8: Beam/Child Diagram 


\subsubsection{Per-Beam Queues}

As is the case for ESIF and HMAC, we set up a separate packet queue for each beam. This prevents HOL blocking. When a higher layer packet arrives at the MAC level, the controller sub-layer, which is hosted by the parent process, checks its address, and forwards it to the appropriate child process (at the sector sub-layer) that then queues the packet on its own queue. For that to be feasible, the parent process maintains a neighbor table as we explain in Section 4.6.8.

\subsubsection{Broadcasting}

Broadcasting is performed in a "decoupled" manner as follows. The controller sub-layer makes $N-1$ copies ( $N$ being the number of sectors) of the packet to be broadcast. The controller forwards each copy to a different sector process. The latter places the packet copy on its queue. That packet is eventually transmitted when it is its turn (as there might be some other packets ahead of it in the queue) and the medium is free in the concerned direction. No RTS/CTS or IFS are required. With this scheme, we can have broadcast packets be transmitted on some beams while unicast packets are transmitted on other beams during a given Tx mode. We call it "diversity-casting", and it is a novelty of MBA-DbMAC. The mechanism is different than in DbMAC since there is no beam-sweeping involved here, and each beam has its own queue. Moreover, in DbMAC, we provided the nodes with the ability to attempt broadcast packet transmissions twice in a given direction (if the medium was found to be busy the first time around). We do not keep this feature in MBA-DbMAC. Rather,

as mentioned, each beam sends its broadcast packet copy independently whenever the medium clears in that direction. With diversity-casting, there is no giving up after one retry as is the case in DbMAC. Therefore, we ensure that all broadcast packets are sent in all directions, unless the normal max retry limit (same as for unicast packets) is reached on a given direction. This should, among others, have a positive impact at the Network layer with route discoveries. The authors of MMAC-NB, ESIF, and HMAC do not specify how broadcasting is handled. But, judging from the emphasis they place on enforcing strict CPT, we can only assume that broadcasting is not decoupled, and that it is done in a synchronous manner; with all the delays that this may impose when confronted with busy medium in certain directions. 
The cost of diversity-casting in terms of energy is the same as for broadcasting with omnidirectional antennas when we set the transmission range of the MBAs to be the same ${ }^{2}$ as for omnidirectional antennas. That is because, following Equation 2.1 and given that the transmission gain on each sector is $n$ ( $n$ being the number of sectors), the transmitter at each sector has a transmission power that is $1 / n$ times that of the omnidirectional antenna of the same range. Therefore, when all $n$ sectors transmit, the total power used is equal to that used in a single transmission of the

omnidirectional antenna. MBAs, however, allow spatial reuse. Besides, we have no reason to believe that having neighbors of a node receive a broadcast packet at different times is going to be an issue. Rather, diversity-casting ensures that the packet is sent to a specific direction as soon as possible instead of waiting for all directions to be clear. This should have a positive impact in delay reduction.

\subsubsection{RTS/CTS}

Similar to DbMAC, RTS and CTS are sent directionally. As the controller sublayer forwards the packet to the appropriate sector process, the latter takes care of it (including the RTS/CTS exchanges) in its own direction, independently from the other sectors/directions. As explained in Chapter 2, this mechanism combats the hidden-terminal problem. Moreover, having the RTS/CTS exchange occur directionally solves another problem that might arise in the context of MBAs. A source node might be engaged in an RTS/CTS exchange with a given neighbor in one direction/beam. Another neighbor that can reach this source node on a different beam does not need to know about this exchange since it can engage in a parallel exchange with the same source node thanks to the CPR capability. Having RTS/CTS exchanged omnidirectionally would unnecessarily block exchanges with neighbors from other beams. This can be seen as an exposed-terminal problem in reverse, specific to MBA environments. Restricting RTS/CTS exchanges to occur directionally solves this potential problem.

\footnotetext{
${ }^{2}$ For fairness in comparison.
} 


\subsubsection{DNAV}

The Directional Network Allocation Vector (DNAV) is similar to the NAV described in the original IEEE 802.11b DCF MAC; except now the NAV is kept on a per-antennabeam basis. The exposed-terminal problem is taken care of through the DNAV. Only the sector that receives the unintended RTS/CTS will be "blocked" for transmission for the duration of the neighboring/overheard transmission. Any other sector of the neighbor can engage in a concurrent transmission/reception. As already noted, there is still the possibility of an exposed-terminal if the parallel transmission falls within the blocked beam (Figure 4.4). But we assume that this is infrequent enough to not justify a separate mechanism to deal with it. For instance, assuming an $N$-sector antenna, assuming that the nodes are uniformly distributed in the network, another node (the one sending the unintended RTS/CTS) has to be in the same sector as the one we would transmit in. That cuts down the probability of this occurring by a factor of $1 / N$. And the impacted node would need to, presumably, use the impacted sector for its transmission, and that would cause another $1 / N$ reduction. Moreover, the sender of the RTS/CTS would also have to use a specific sector (out of its own $N$ ) that points to the impacted node. That is yet another $1 / N$ reduction. In the end, we can see that that would imply a reduction by $1 / N^{3}$ of the probability of occurrence of the exposed-terminal problem. Therefore, that probability drops considerably with an increase in the number of sectors $N$.

\subsubsection{Neighbor Table}

Broadcasting is an important and frequently exploited communication primitive. In the broadcasting mode, nodes send packets on all sectors without having to know where neighbors are. In fact, numerous network layer protocols (routing) do perform neighbor discovery through periodic HELLO message exchanges which are one-hop broadcast messages. Moreover, on-demand protocols broadcast Route Discovery messages before sending unicast packets. Broadcasting messages offer the opportunity to a node to "advertise" itself to all the nodes within its transmission range, and hence be included in their neighbor tables.

Similar to DbMAC, each node maintains a neighbor table. This table is maintained by the controller process with updates coming from individual sector processes. 
The neighbor table is initially empty and is progressively populated as follows. Whenever a sector receives a frame from the Physical layer, the process responsible for that sector informs the controller sub-layer of the address of the sender of that frame. The controller then updates the neighbor table, if needed, with information that says "Neighbor $A$ lies on beam $i$ ". That way, if a frame is later to be sent to that neighbor, the node (controller) knows which antenna sector to use. Therefore, we do not have to calculate the direction of arrival; we know it as soon as the frame arrives on a given sector (that represents a good-enough approximate direction).

\subsection{MBA-DbMAC Functionality Testing}

In this section, we perform functionality checks of MBA-DbMAC. These functionality checks include: uni-packet transmission (UPT), broadcasting, concurrent-packet transmission, and concurrent-packet reception. Furthermore, we examine the time spent in transmit mode and in receive mode. Finally, we assess the performance of our protocol against that of IEEE 802.11b (omnidirectional antenna) and DbMAC (single-beam directional antenna). This performance is evaluated in terms of delay, throughput, and goodput. For these tests, we use the static star-topology scenario depicted in Figure 4.9 where node 1 is the central node and all other nodes are peripheral. The default data packet generation rate is set to 5 packets/sec. However, we will increase (and specify) this rate occasionally for some of the tests. All the tests are conducted using OPNET Modeler 16.0, the platform/simulator introduced earlier in Chapter 2, on which we have implemented our protocol. The channel capacity is 11 Mbps, and the default simulation time is 1800 seconds.

\subsubsection{Uni-Packet Transmission Test}

We perform a basic test of uni-packet transmission. Node 1 sends packets to node 2 only. The results are shown in Figure 4.10.

The first sub-graph shows that about 5 packets per seconds are sent from node 1. The remaining sub-graphs clearly show that only node 2 receives all 5 packets per second sent by node 1, which is the only source. The other nodes do not receive 


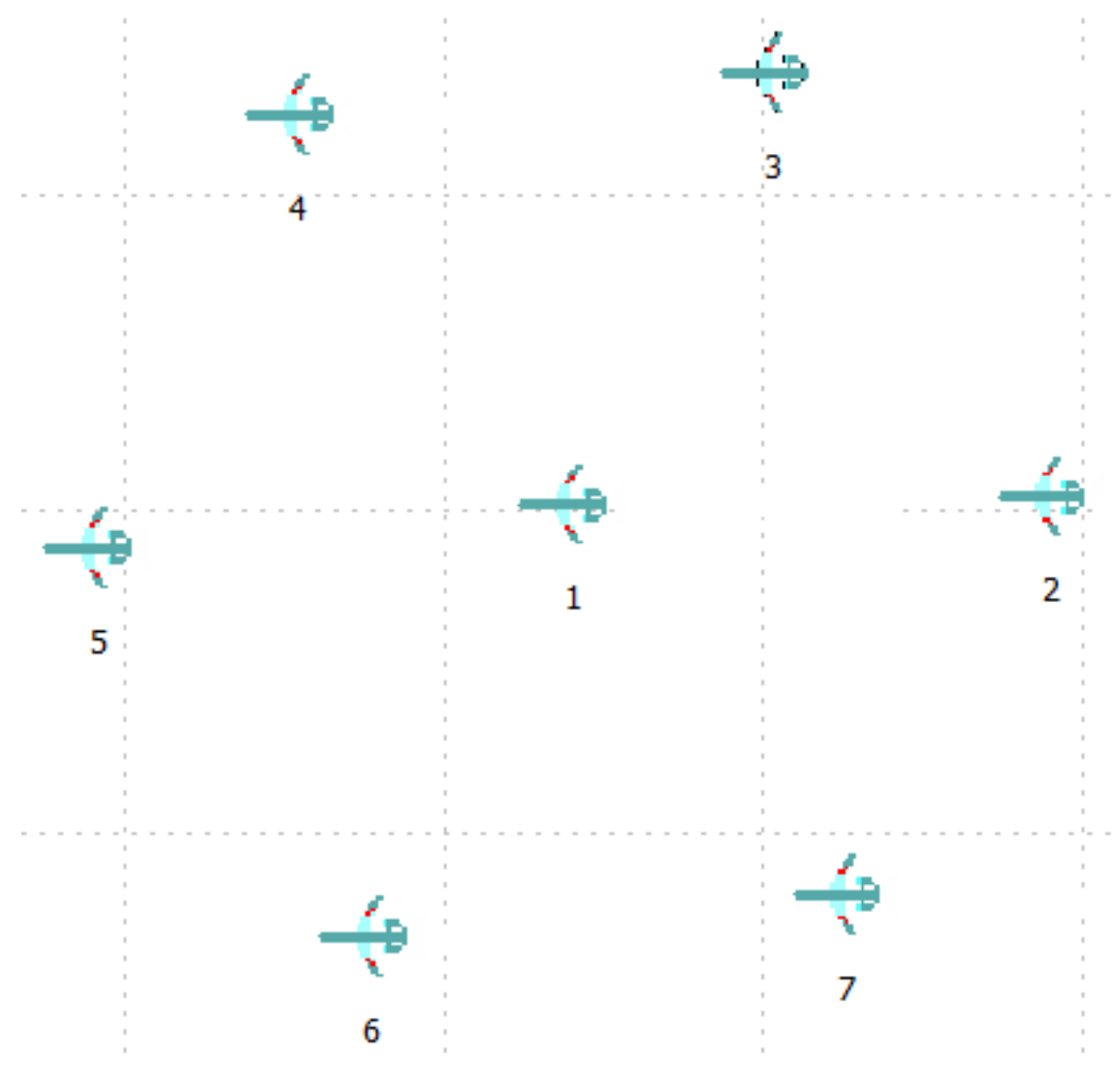

Figure 4.9: MAC Test Scenario Topology

anything as no packet from node 1 is addressed to them. This shows that the basic uni-packet transmission of MBA-DbMAC protocol is working.

Note that, since we are dealing with unicast packets, ACK packets are sent by the destination. Also RTS/CTS exchanges are made for packets that are larger than a certain threshold. Therefore, in reality, node 1 does not only act as a transmitter of data (and RTS) packets, but it also acts a receiver of ACK and CTS packets. Likewise node 2 does not only act as a mere receiver of data (and RTS) packets, but it is also a transmitter of ACK and CTS packets. Both nodes experience Tx and Rx modes, though only one beam is involved in each node.

\subsubsection{Broadcasting Test}

We perform a basic test of broadcast transmission. Node 1 broadcasts packets. The results are as shown in Figure 4.11. 

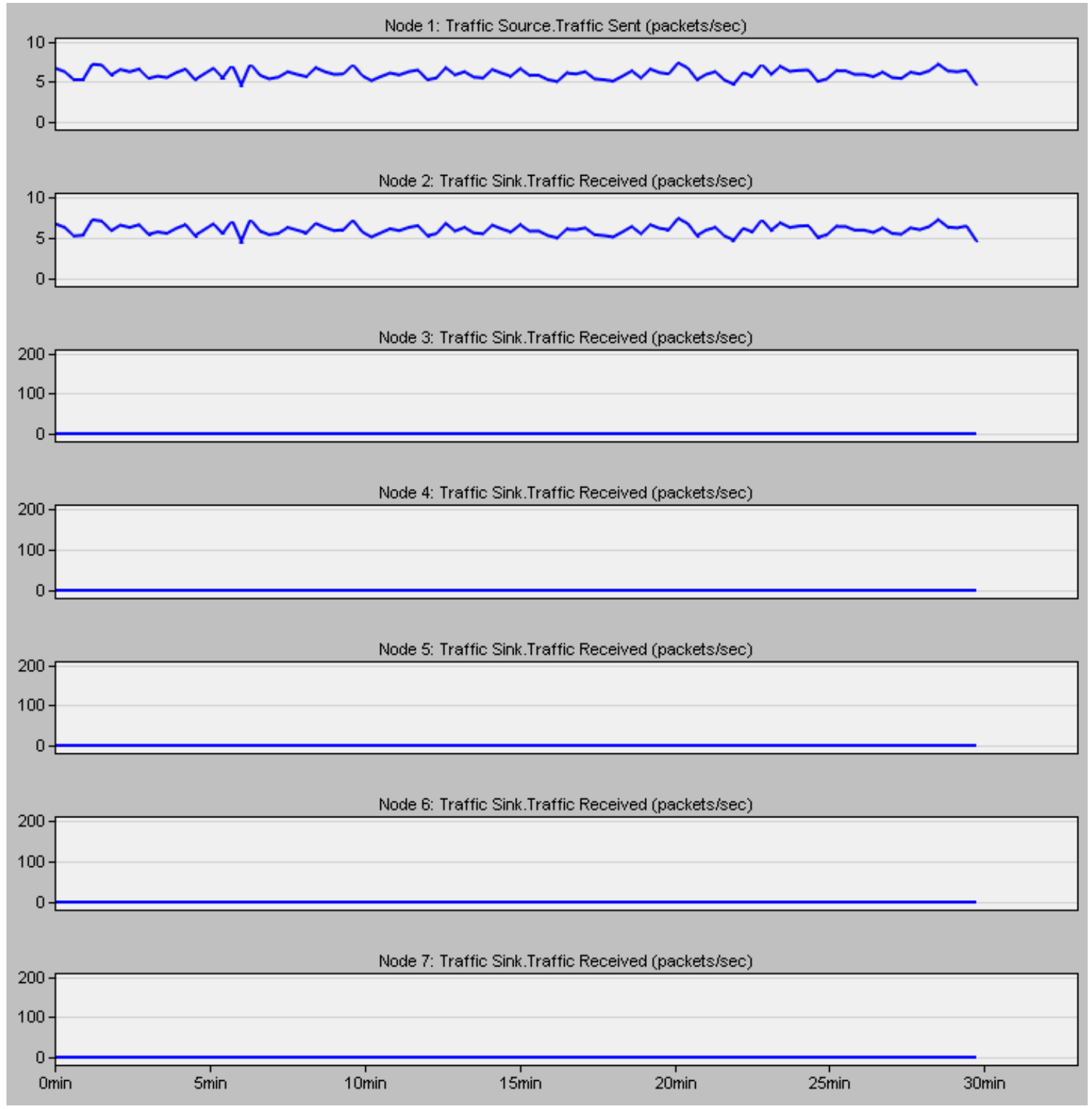

Figure 4.10: UPT Test

The first sub-graph shows that approximately 5 broadcast packets per seconds are sent from node 1 . The remaining sub-graphs clearly show that all nodes receive 5 packets per second sent by node 1 , the only source. This shows that the broadcasting of MBA-DbMAC is working.

With this scenario, node 1 only operates in Tx mode, and all the other nodes only 


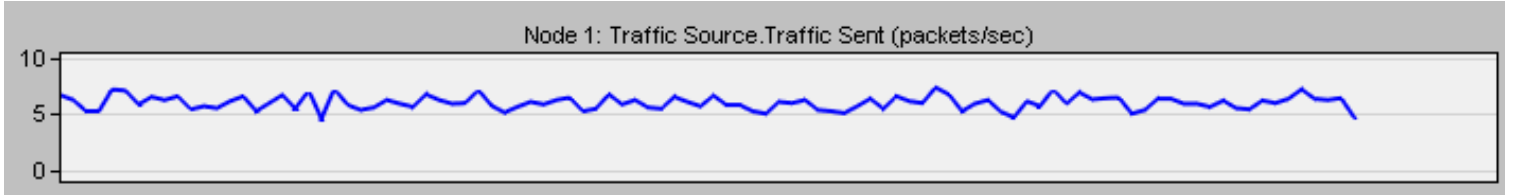

Node 2: Traffic Sink.Traffic Received (packetsisec)

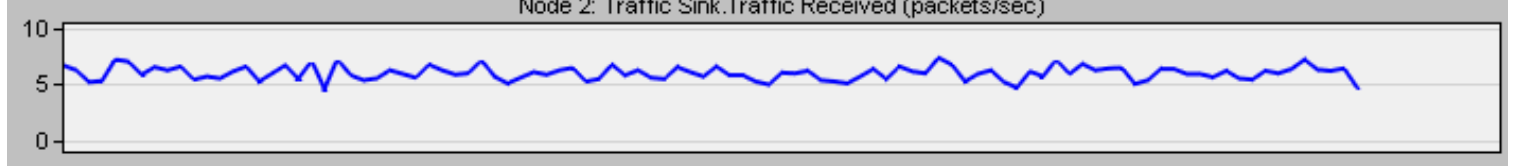

Node 3: Traffic Sink.Traffic Received (packetsisec)

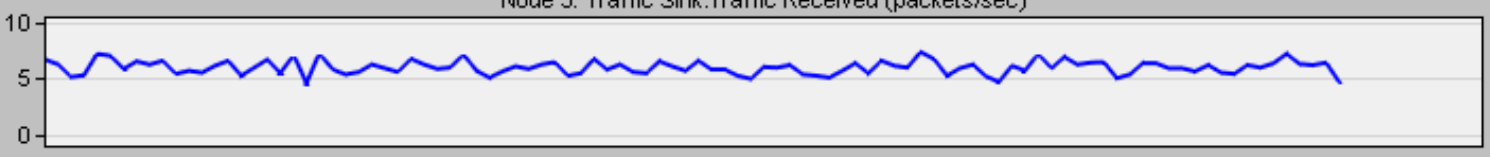

Node 4: Traffic Sink.Traffic Received (packetsisec)

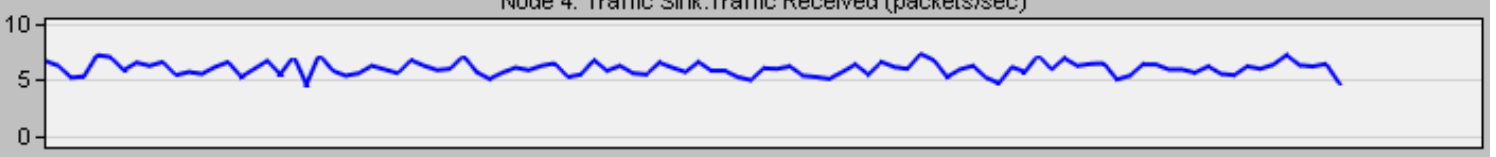

Node 5: Traffic Sink.Traffic Received (packetsisec)

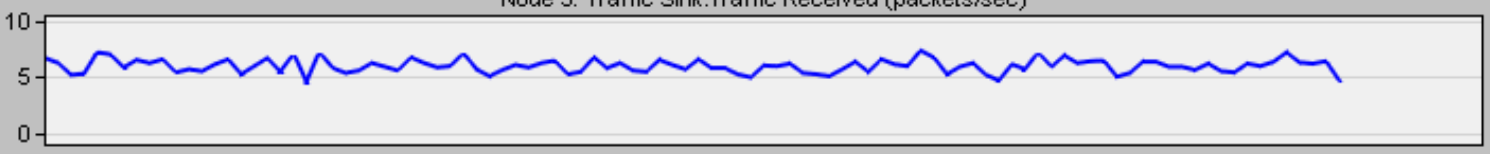

Node 6: Traffic Sink.Traffic Received (packetsisec)

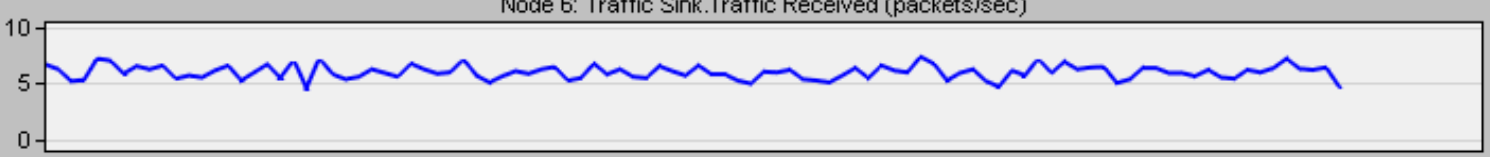

Node 7: Traffic Sink.Traffic Received (packetsisec)

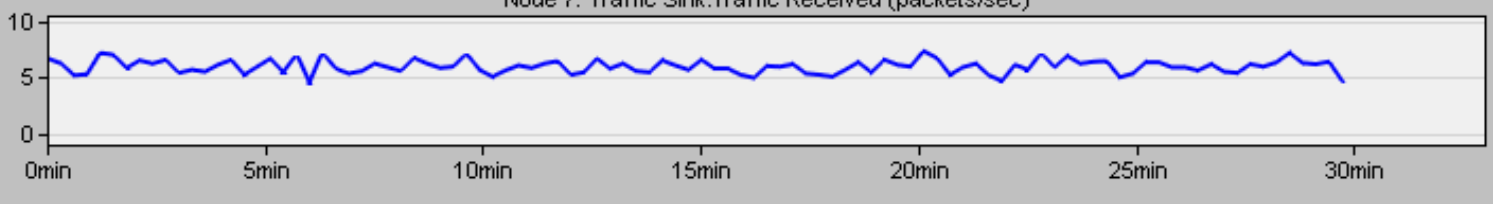

Figure 4.11: Broadcast Test

operate in Rx mode. Of course, Idle mode does occur, but we only focus on $\mathrm{Tx} / \mathrm{Rx}$ mode functionality. Node 1 utilizes all its 6 antenna sectors.

\subsubsection{CPT-CPR Test}

To test CPT-CPR, node 1 sends and receives unicast data packets from nodes 2, 3, 4, 5, 6, and 7. These nodes all start sending data packets to node 1 around the same 
time. For the CPT functionality, the results are as shown in Figure 4.12.

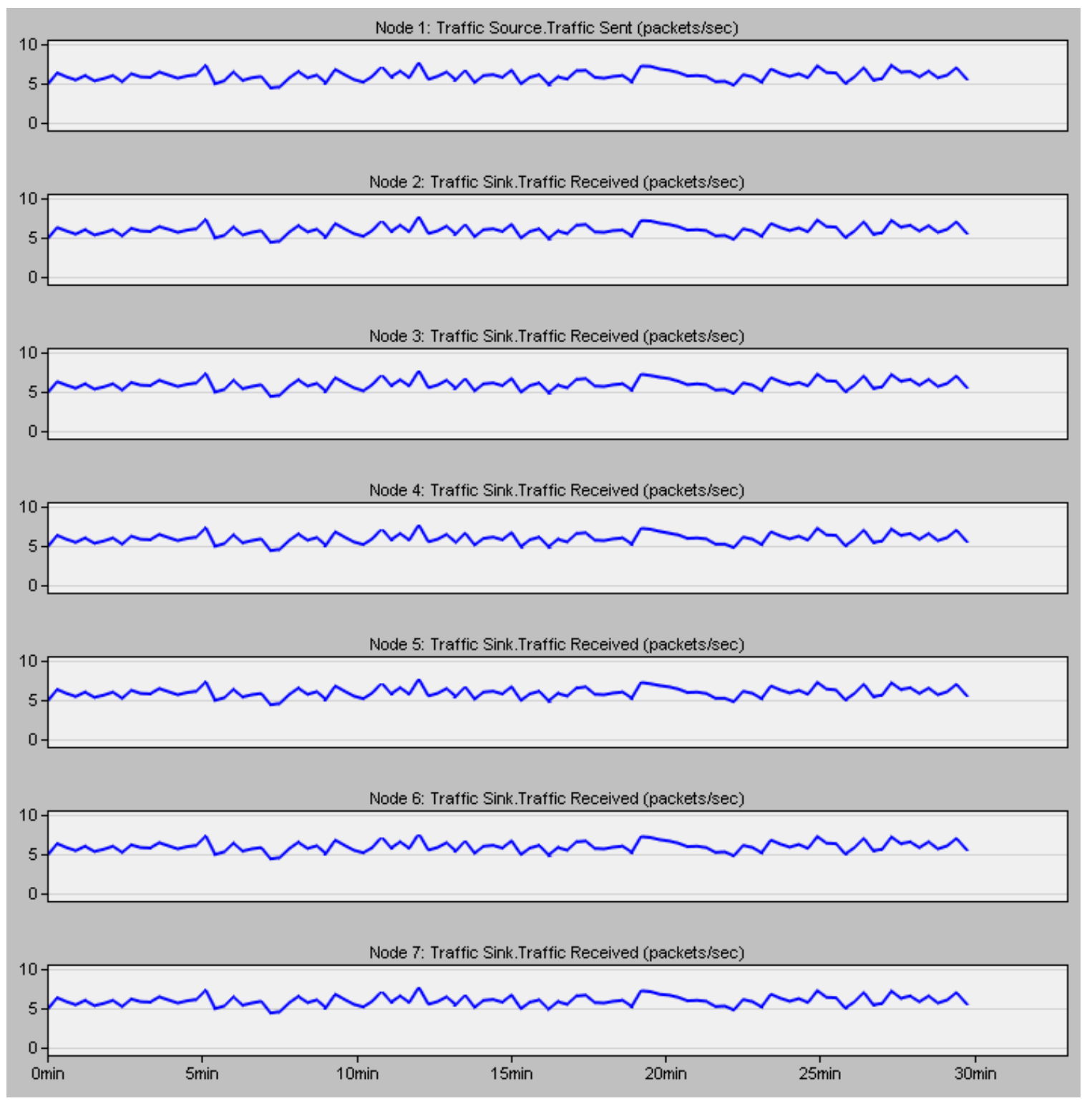

Figure 4.12: CPT Test

The first sub-graph shows that a little over 5 packets per seconds are sent from node 1. This rate is actually the rate at Application-layer level. However, in order to test CPT at MAC level, five copies are made for each Application-layer packet (making it a total of 6 packets to be sent at MAC level, one for each sector). For each copy, a different destination address (corresponding to a different neighbor) 
is set, and the packet is sent via the sector that points to the intended neighbor. Therefore, the Application-layer rate becomes the per-sector rate at the MAC level. We implemented this scheme this way because OPNET does not allow us to issue application-layer-level packets with different destination addresses. The remaining sub-graphs clearly show that all nodes, each lying on a different beam of node 1 , receive 5 packets per second sent by node 1 on the concerned beam. This is the first step in showing the CPT functionality of MBA-DbMAC.

For the CPR functionality, we obtain the results shown in Figure 4.13. The first sub-graph shows that about 35 packets per seconds are received by node 1 . The remaining sub-graphs show that nodes $2,3,4,5,6$, and 7 each send a little over 5 packets per second; thus the total of about $35^{3}$ packets received by node 1 . This is the first step in showing the CPR functionality of MBA-DbMAC.

\subsubsection{Rx Mode vs Tx Mode}

We measure the time spent in Rx and Tx mode in the situation of CPT/CPR. The traffic rate is still 5 packets per second. For a peripheral node, the time spent in $\mathrm{Rx}$ mode (18 seconds) and in Tx mode (18 seconds) is balanced since only one beam is involved. However, for the central node (node 1), we obtain that more time is spent in Rx mode (79 seconds) than in Tx mode (48 seconds). This imbalance can be explained by the fact that, with our current rate of packet generation ( 5 packets per second), there are more instances of the central node not having packets to transmit than there are instances of at least one of the six peripheral nodes not having packets to transmit (hence the central node not receiving).

One way to address/overcome this asymmetry is to increase data packet generation so that the output buffer of all the nodes is always full. For example, with a higher packet generation rate of $20 \mathrm{pkts} / \mathrm{sec}$, we obtain a more symmetric result for the central node of 304 seconds spent in Rx mode and 298 seconds spent in Tx mode.

These time results are the final step in showing that we do, in fact, have CPR/CPT

\footnotetext{
${ }^{3}$ Each of the 6 senders sends a little over 5 pkts/sec, more like a little under 6 pkts/sec, therefore the total received at the common receiver is greater than 30 packets, but still less than 36 packets (as it would be if the senders' rate were exactly $6 \mathrm{pkts} / \mathrm{sec}$ ).
} 


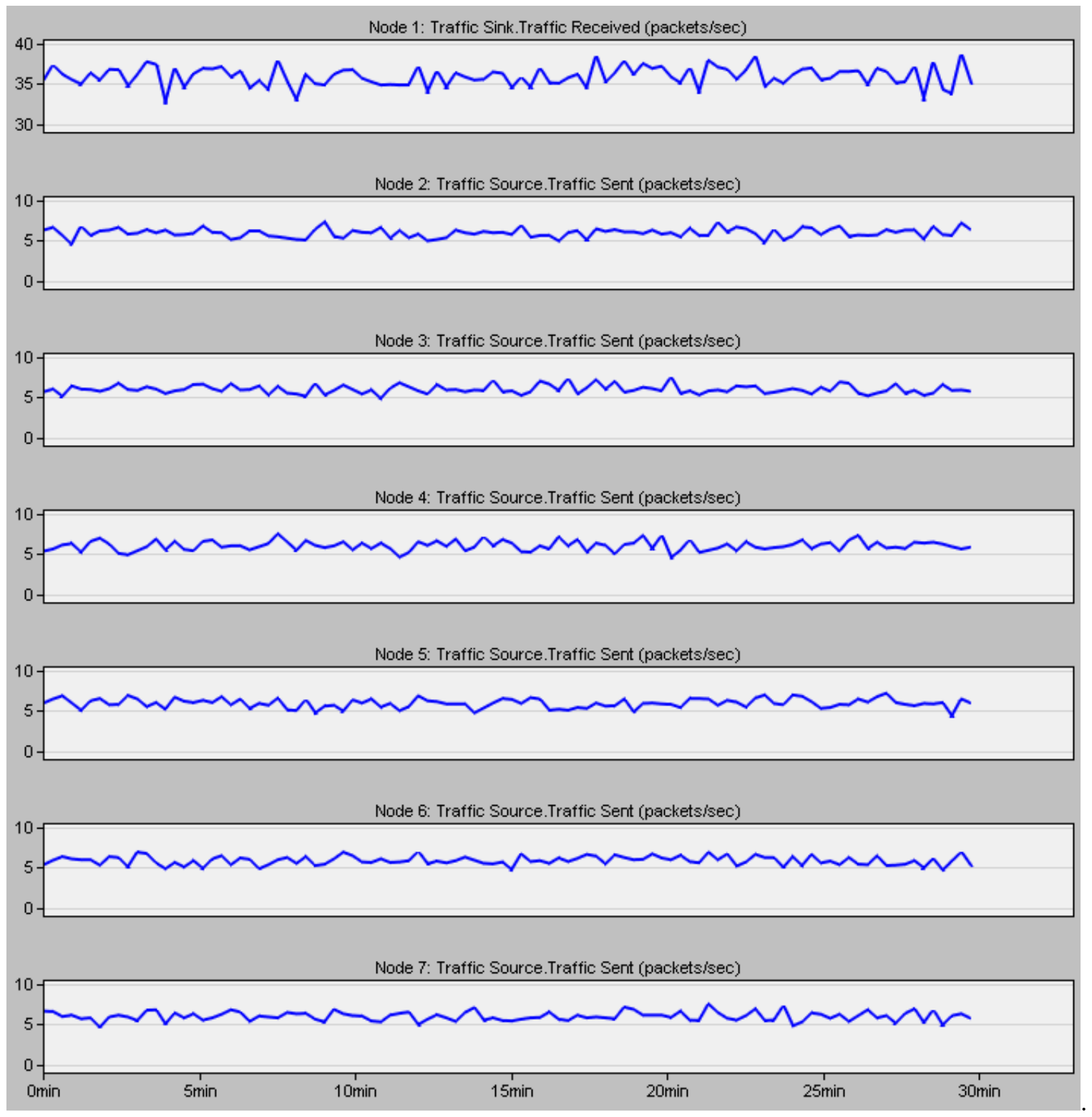

Figure 4.13: CPR Test

functionality. If the 6 peripheral nodes spend collectively $6 \times 18$ seconds to transmit, that is 108 seconds. But, as we saw earlier, the central receiver (node 1) spends only 78 seconds receiving. This means that some of the receptions need to happen in parallel; because, after all, all packets are received, as we saw in Figure 4.13. This therefore shows the CPR functionality of MBA-DbMAC. Similarly, if the central node sent its packets sequentially (one beam at a time) it would behave as a peripheral node (single beam used) in that regard; hence it would take a total of about $18 \times 6=108$ 
seconds to complete its transmissions. But, as seen earlier, it only takes 47 seconds, with no packet lost (Figure 4.12); which means that some transmissions have been done in parallel. This therefore shows the CPT functionality of MBA-DbMAC.

\subsubsection{Performance Comparison: Delay, Throughput, and Goodput}

With the same star topology, we compare the MAC delay, throughput, and goodput when three types of antennas are used: MBAs, SBAs, and omnidirectional antennas. In any given scenario, all nodes are equipped with the same antenna type. MBAs work with the MBA-DbMAC protocol, SBAs work with the DbMAC protocol, and omnidirectional antennas work with the IEEE 802.11b DCF MAC protocol.

The delay here is measured from the time when a packet is inserted into the transmission queue until it is received. It includes the period for the successful RTS/CTS exchange, if this exchange is used prior to the transmission of that frame.

The network throughput is defined as the total number of bits (in bits/sec) forwarded from wireless LAN layers to higher layers in all the WLAN nodes of the network. The network goodput is defined as the application-level throughput (i.e. the number of useful information bits delivered by the network to a certain destination per unit of time). The amount of data considered excludes protocol overhead bits as well as retransmitted data packets.

The performance in terms of the delay is presented in Figure 4.14. The observation is as follows: the smallest delay is experienced with MBA antennas, $0.5 \mathrm{~ms}$, compared to $3.5 \mathrm{~ms}$ for Omni, and $5.5 \mathrm{~ms}$ for SBA antennas. MBA antennas reduce the delay by about a factor of 10 . The delay of SBA being significantly worse than Omni illustrates that deafness is a more severe issue than the exposed-terminal problem in this configuration. In effect, we know (from Chapters 2 and 3) that SBAs are more affected by deafness than any of the other two types, whereas omnidirectional antennas are more affected by the exposed-terminal problem. 


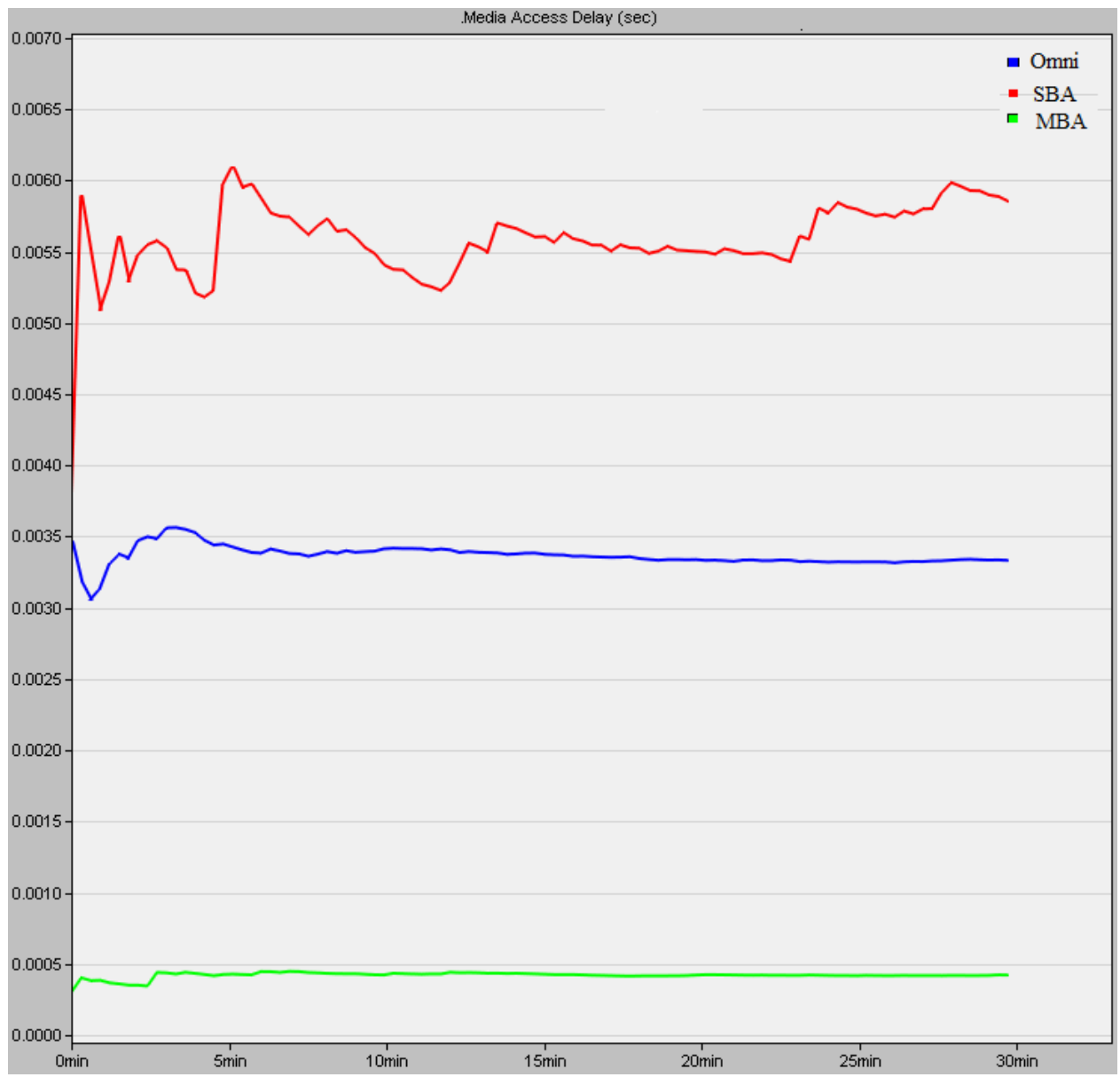

Figure 4.14: MAC Delay, with 5 pkt/sec Traffic Generation Rate

As shown in Figure 4.15, the throughput is similar for all three types of antennas. This can be explained by the fact that the nodes spend most of their time in Idle mode (no transmission, no reception). For instance, in the case of MBA-DbMAC testing, we saw earlier that the central node spends a total of about 126 seconds in transmission and reception combined (out of 1800 seconds of simulation). Peripheral nodes spend about 36 seconds out of 1800 in transmission and reception combined. These times in active mode are expected to be higher in the case of SBA and omni antennas (since there is no concurrence of multiple transmissions or receptions). But since we still receive all the sent packets notwithstanding the type of antenna used, it makes sense to have the same throughput.

If we augment the packet generation rate to $105 \mathrm{pkt} / \mathrm{sec}$, we now have a total 


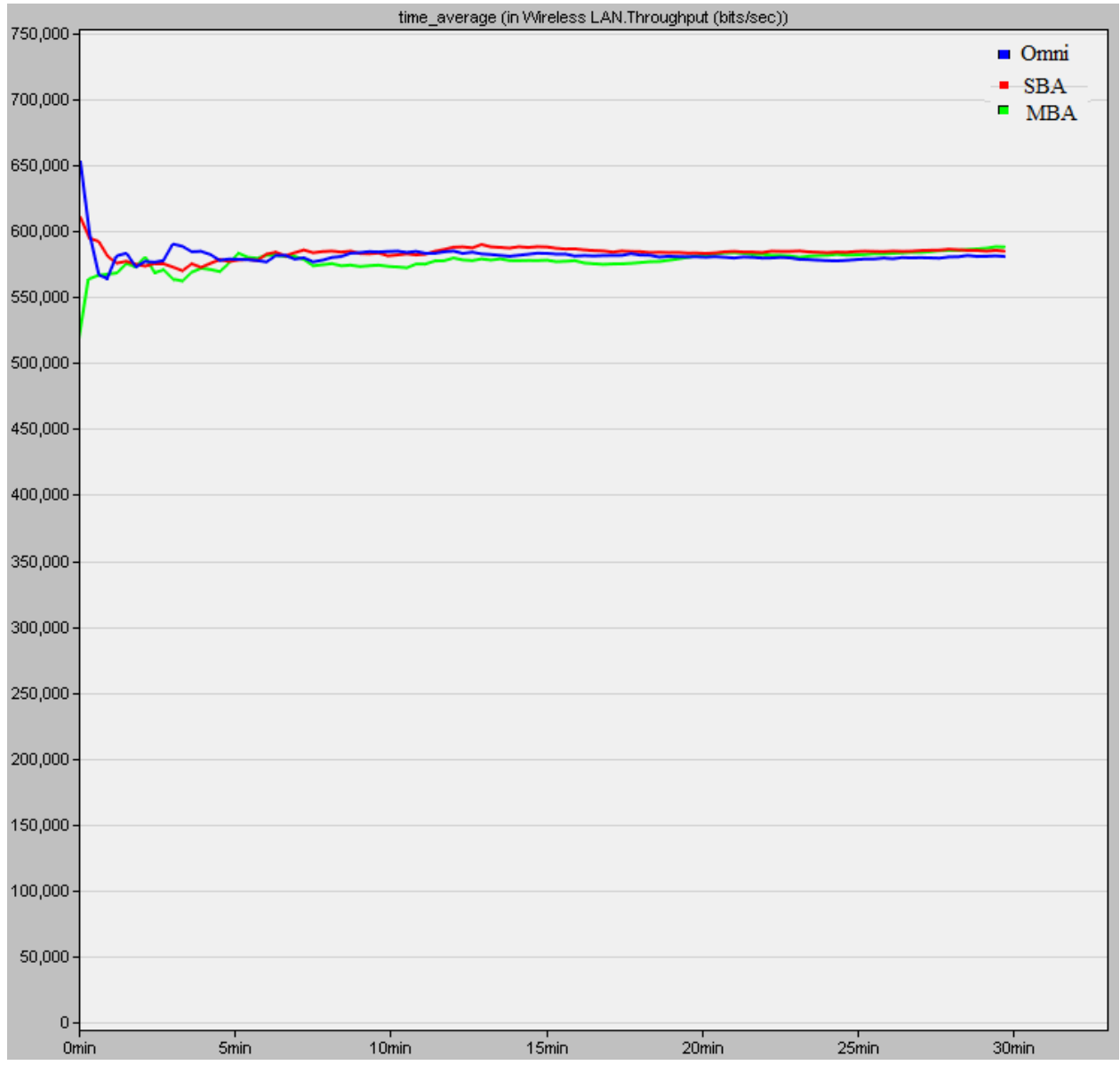

Figure 4.15: Throughput, with $5 \mathrm{pkt} / \mathrm{sec}$ Traffic Generation Rate

network load of at least $105 \mathrm{pkt} / \mathrm{sec} \times 1024$ Bytes/pkt $\times 8$ bits/Byte $\times 12$ nodes ${ }^{4}$ $=10.3 \mathrm{Mbps}$ (recall that the channel capacity is set to $11 \mathrm{Mbps}$ ). The throughput is shown in Figure 4.16. SBA fails completely with this high packet rate. MBA has a lower throughput than Omni. However, as Figure 4.17 shows, MBA actually shows a higher goodput (actual data packets received at the peripheral nodes); even though, in the absolute, both types of antennas (and their corresponding MAC protocols) perform poorly with packet delivery ratios (goodput) of $40 \%$ and $50 \%$ respectively.

\footnotetext{
${ }^{4} 12$ nodes $=6$ peripheral nodes +1 central node. However, the central node acts as 6 sources (each of its 6 sectors sends traffic to a neighbor) of traffic.
} 


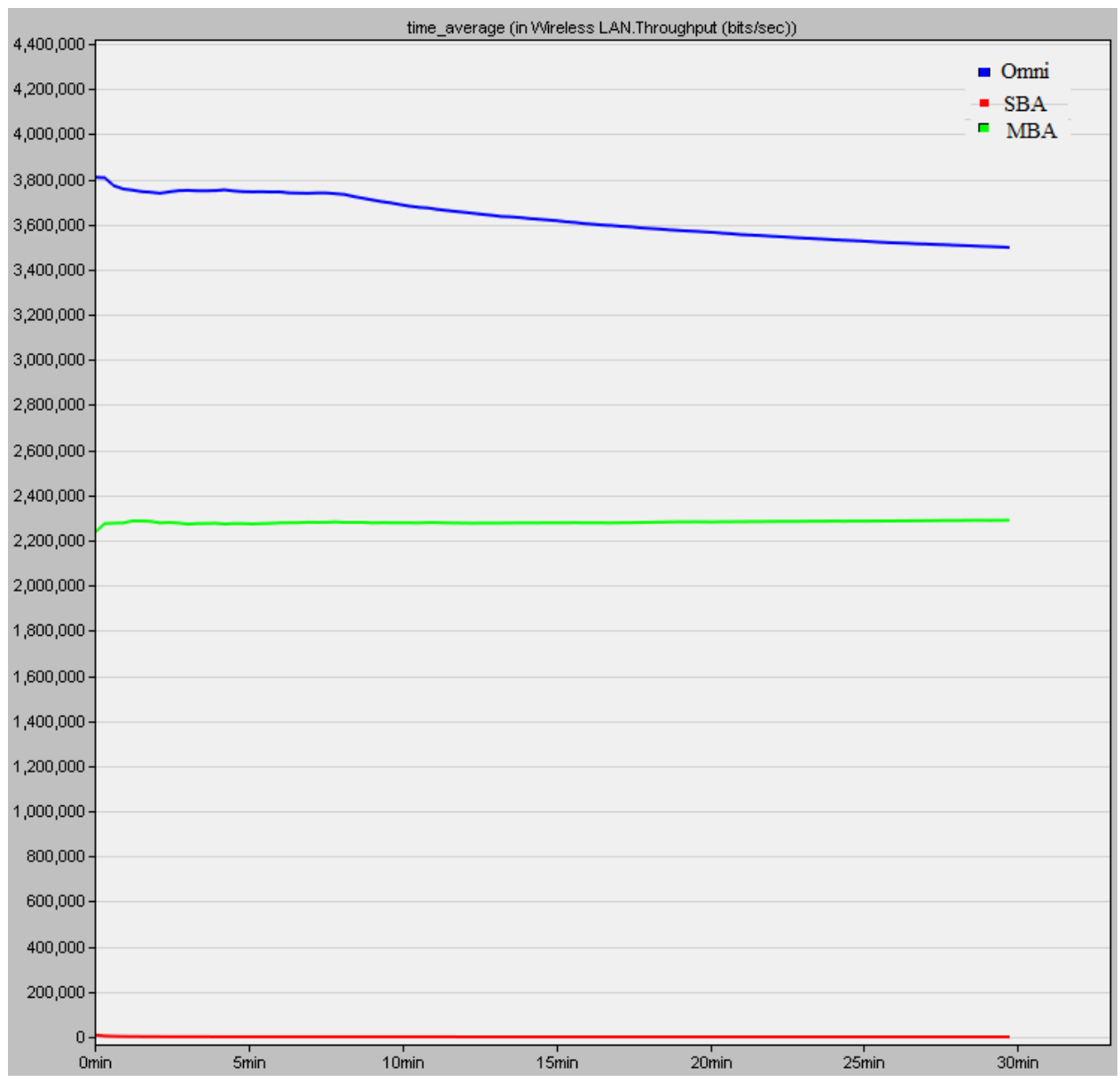

Figure 4.16: Throughput, with 105 pkt/sec Traffic Generation Rate

\subsection{Summary}

The proposed MBA-DbMAC protocol is a generic MAC protocol that has the basic functionalities of a MAC protocol and renders possible the basic operation of MBAequipped nodes. MBA-DbMAC is an adaptation/extension of DbMAC to the MBA environment. ESIF, HMAC, and MMAC-NB are all MAC protocols designed to work with MBAs. We used the design of these protocols as a starting point in the identification of MBA-specific issues inherent to MAC design. We have adopted some ideas from these protocols, but we are also proposing different, innovative, and fairly simple solutions to some MBA-specific issues. For instance, for the design of the MBA-DbMAC protocol, we adopt a two-tier processing approach whereby the MAC layer is split into two artificial sub-layers: the controller sub-layer (materialized by one node-wide parent process) and the sector sub-layer (materialized by $N$ child processes, 


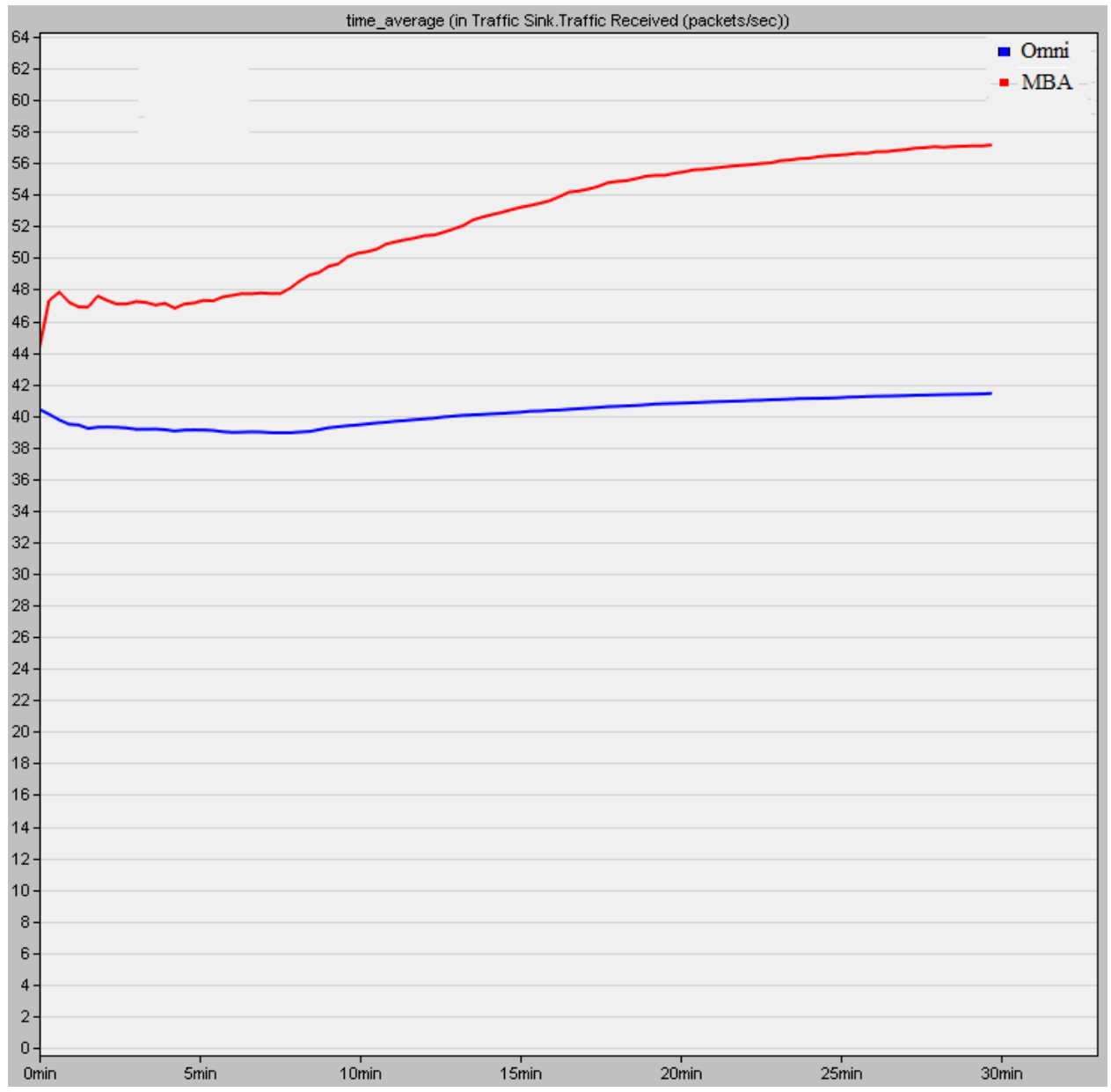

Figure 4.17: Goodput, with 105 pkt/sec Traffic Generation Rate

1 child process for each of the $N$ sectors). The sector sub-layer implements traditional MAC-layer mechanisms while the controller sub-layer manages: a) the neighbor table, b) the assignment of a high-layer packet to the appropriate sector, and c) the switch from/to the different operation modes (Tx mode, Idle mode, and Rx mode). This two-tier design approach is an elegant way of enforcing mode switches in the node while still leaving the full autonomy of medium access to sectors in their respective direction of competence. Other novel aspects of our solution are the decoupledbroadcasting or diversity-casting and the time window policy that we adopt in order to avoid Critical Chain Transmission and Critical Chain Reception. In the end, our goal was to have a functional MBA-specific MAC protocol to work with while we tackle delay-reducing routing issues at the Network level; the main focus of our work. With this MBA-DbMAC protocol, we believe that we have reached that goal and are 
now ready to take on routing issues. 


\section{Chapter 5}

\section{An Optimization Model for End-to-End Delay Reduction in Ad Hoc Networks}

\section{$5.1 \quad$ Introduction}

As already noted, MBA nodes have two main characteristics: the MPT capability and the MPR capability, whereby a node can transmit/receive multiple packets at the same time. The antenna is either in transmit mode or in receive mode, not both at the same time, due to half-duplex constraints. In this chapter, we provide an analysis of how this MPT/MPR capability can be used to reduce the E2E delay for delivered packets in ad hoc networks. We formulate the delay reduction issue as an optimization problem that we solve.

In the literature (see Chapter 3), the work around MBAs has focused on designing MAC and/or routing protocols that exploit spatial reuse in order to increase network performance metrics such as throughput and packet delivery ratio. Not much has been done to use the full MPT/MPR potential for E2E delay reduction. Moreover, a great deal of existing work on MBAs is done for infrastructure-based networks with an applicability to ad hoc networks that is yet to be clarified. Furthermore, several link scheduling proposals for network performance improvement do not consider MBAs at all.

Our ultimate goal is to design a routing protocol that exploits MBAs in order to minimize/reduce the E2E delay in ad hoc networks. We ask ourselves: a) How can we benefit from the MPT/MPR capability of MBAs? And b) What should the path 
selection criteria be in the context of MBAs for E2E delay minimization/reduction?

In this chapter, we make the case that the full potential of the MPT/MPR capability of MBAs can be unlocked to significantly reduce the end-to-end delay in static ad hoc networks. We define a formal optimization model for delay reduction, and we learn some key routing-related lessons by solving it. In effect, we observe that the optimal end-to-end delay is attained when links are scheduled in such a way that opportunities for MPT/MPR are maximized. This results in a selection of routes that, up to half of the time, are not the shortest. We actually show that using only the shortest routes, a widespread criterion in traditional routing protocols for ad hoc networks, results in higher delays. To the best of our knowledge, no such analysis of the potential of MBAs to reduce/minimize the delay has been conducted thus far for ad hoc networks. Though our optimization model is developed around static ad hoc networks, the goal is to learn some key lessons from solving the model; lessons that we will exploit to a great extent in mobile networks. The model proposed in this chapter is not a routing solution/protocol. Rather, the model is a way to learn about the properties of good (low-latency) routes.

The remainder of this chapter is organized as follows. Section 5.2 presents our optimization model. The results from solving this model are discussed in Section 5.3. These two sections assume a "beam-less" or "beam-transparent" model. In other words, the assumption is that each link in the network has a dedicated beam (there cannot be more than one neighbor within any given beam of any given node). In Section 5.4, we refine the model by adding the notion of beams to it (there can now be many links or neighbors within a single beam). This addition makes our model more realistic. Concluding remarks of the chapter are provided in Section 5.5.

\subsection{Scheduling Problem Formulation}

\subsubsection{Assumptions}

We assume the following conditions:

- All nodes in the network are perfectly time-synchronized.

- The nodes run a perfect TDMA-based MAC protocol for MBA antennas. Time 
is divided in time slots.

- Multiple nodes can transmit in the same time slot if their transmissions do not interfere with one another.

- Nodes operate in half-duplex mode: a given node cannot transmit and receive at the same time.

- A node can receive up to $M$ simultaneous packets (MPR capability), or perform up to $M$ simultaneous transmissions (MPT capability) at a time.

- Each link has a dedicated antenna beam/sector; we make the notion of beam transparent for now. It will be taken into account in a later iteration (in Section 5.4).

- All nodes have the same antenna characteristics (transmission/reception range, beamwidth, etc.). This antenna homogeneity assumption makes the radio links perfectly symmetrical: if node $i$ can hear node $j$, then node $j$ can also hear node $i$. Note that this antenna homogeneity is not a requirement for our model to work, although it simplifies it a little bit. It is possible to refine the model for antenna-heterogeneous networks.

\subsubsection{Optimization Model}

For a given static network with multiple flows, and provided that the antennas are MPT/MPR capable, we would like to know the route selection (link scheduling) that gives us the lowest end-to-end delay (average) possible. This is an optimization problem. We represent the network with a directed connectivity graph $G(V, E)$, where $V$ represents the set of nodes (or vertices) in the network, and $E$ is the set of directed links (or edges). If node $i$ is within the reception range of node $j$, then links $(i, j)$ and $(j, i)$ are members of $E$ (see the antenna-homogeneity assumption in Section 5.2.1).

The inputs of our problem are:

- The connectivity graph $G(V, E)$.

- The MPR/MPT capability $M$. 
- The number of traffic flows $|F|$, i.e. the cardinality of the set $F$ containing the numbers that identify the flows. $F=\{1,2, \ldots,|F|\}$.

- The source and destination of each flow. $s_{f}$ and $d_{f}$ are respectively the source and the destination of flow $f$, with $f \in F$.

The outputs are:

- The selected route for each flow, presented as a sequence of links.

- The delay (in number of time slots) of each route. The average delay per route is then the sum of all delays divided by the number of flows.

Table 5.1: Notation for MBA-DM-1

\begin{tabular}{ll}
\hline$s_{f}$ & Source node for flow $f$ \\
$d_{f}$ & Destination node for flow $f$ \\
$\alpha_{i j}^{f k}$ & Scheduling status of link $(i, j)$ of flow $f$ at time slot $k$ \\
$\alpha_{i d_{f}}^{f k}$ & Scheduling status of link $\left(i, d_{f}\right)$ of flow $f$ at time slot $k$ \\
$j \mid(i, j) \in E$ & Node $j$ such that $(i, j) \in E$ \\
$j \in V \mid(i, j) \in E$ & Node $j \in V$ such that $(i, j) \in E$ \\
\hline
\end{tabular}

In order to solve this optimization problem, we define a decision variable $\alpha_{i j}^{f k}$ that tells us whether or not link $(i, j)$ of flow $f$ is scheduled at time slot $k . \alpha_{i j}^{f k} \in\{0,1\}$. $T$ is the set of time slots. $T=\{1,2, \ldots,|E| \times|F|\}$, where $|E|$ is the cardinality of $E$. The $T$ set is constructed assuming that each flow uses all the available links (edges) and each link from each flow in the network gets its own time slot and there are no concurrent transmissions (worst case scenario). We designate our optimization problem as MBA-DM-1 (Delay Minimization with MBAs, Version 1). Table 5.1 explains the notation. MBA-DM-1 is formulated as follows:

MBA-DM-1:

Minimize $\sum_{f \in F} \sum_{k \in T} \sum_{i \in V} \alpha_{i d_{f}}^{f k} \cdot k$

subject to the following 6 constraints: 
1. Flow Circulation Constraints

$$
\begin{aligned}
& \sum_{j \mid\left(s_{f}, j\right) \in E} \sum_{k \in T} \alpha_{s_{f} j}^{f k}=1 \quad \forall f \in F \\
& \sum_{i \mid\left(i, d_{f}\right) \in E} \sum_{k \in T} \alpha_{i d_{f}}^{f k}=1 \quad \forall f \in F \\
& \alpha_{i j}^{f k}-\sum_{t=1}^{k-1} \sum_{l \mid(l, i) \in E} \alpha_{l i}^{f t} \leq 0 \quad \forall f \in F, \forall k \in T, \forall i \in V-\left\{s_{f}\right\}, \forall j \in V \mid(i, j) \in E
\end{aligned}
$$

2. Flow Consistency Constraints

$$
\begin{aligned}
& \sum_{l \mid(i, l) \in E} \alpha_{i l}^{f k} \leq 1 \quad \forall f \in F, \forall k \in T, \forall i \in V \\
& \sum_{l \mid(l, j) \in E} \alpha_{l j}^{f k} \leq 1 \quad \forall f \in F, \forall k \in T, \forall j \in V \\
& \sum_{k \in T} \alpha_{i j}^{f k} \leq 1 \quad \forall f \in F, \forall(i, j) \in E
\end{aligned}
$$

3. Link Occupancy Constraint

$$
\sum_{f \in F} \alpha_{i j}^{f k} \leq 1 \quad \forall k \in T, \forall(i, j) \in E
$$

4. Half-duplex Constraint

$$
\sum_{f \in F}\left(\alpha_{i j}^{f k}+\alpha_{j l}^{f k}\right) \leq 1 \quad \forall k \in T, \forall i, j, l \in V
$$


5. MPT Capability Constraint

$$
\sum_{l \mid(i, l) \in E} \sum_{f \in F} \alpha_{i l}^{f k} \leq M \quad \forall k \in T, \forall i \in V
$$

6. MPR Capability Constraint

$$
\sum_{l \mid(l, j) \in E} \sum_{f \in F} \alpha_{l j}^{f k} \leq M \quad \forall k \in T, \forall j \in V
$$

Concerning the objective function from Equation 5.1, the goal is to minimize the total number of time slots required to complete all flows. This is equivalent to finding routes where the last link (link to the destination node) occurs the earliest possible. To that effect, $k$ in the function represents the cost for each time slot. This cost is chosen in order to render prohibitive any scheduling at a later slot. Note that, for a given flow, scheduling the last link the earliest possible is equivalent to minimizing the latency of the entire flow because the last link cannot be scheduled unless the second-last has been scheduled and so on, all the way back to the first link (from the source). Our flow circulation constraints ensure that all necessary links are scheduled in sequence before a given link occurs (is scheduled).

The constraints are explained as follows:

\section{Flow Circulation Constraints}

- Constraint 5.2: a given flow always starts, meaning the source node should be scheduled exactly once for that flow.

- Constraint 5.3: the destination of a given flow must always be reached, by exactly one link.

- Constraint 5.4: link $(i, j)$ can be scheduled for flow $f$ only if $i$ has previously received a packet; meaning there exists a completed $(l, i)$ link from the same flow. Except if $i$ is the source of the flow.

2. Flow Consistency Constraints 
- Constraint 5.5: the same flow cannot have multiple next hops at any given intermediate node belonging to the route.

- Constraint 5.6: the same flow cannot be received from multiple previous hops at any given intermediate node belonging to the route.

- Constraint 5.7: a link can be scheduled only once at most, for any given flow. Note that this constraint does not cover constraints 5.2 and 5.3. The latter two ensure that flows start and terminate, whereas the former simply ensures that a given flow is not scheduled more than once (could be zero) at any given link. This would prevent loops.

3. Link Occupancy Constraint

- Constraint 5.8: only one flow is permitted on a link at a time.

4. Half-duplex Constraint

- Constraint 5.9: transmission and reception cannot occur at the same time at any given node.

5. MPT Capability Constraint

- Constraint 5.10: up to $M$ outgoing links can be scheduled at a node at any time slot.

6. MPR Capability Constraint

- Constraint 5.11: up to $M$ incoming links can be scheduled at a node at any time slot.

\subsection{Model Results and Analysis}

We solved the foregoing optimization model using the Optimization Programming Language (OPL) [97] with IBM-ILOG-CPLEX Optimization Studio 12.7 .1 [98], with all the parameters set to their default values. We worked on an Intel Core it $\mathrm{CPU}$ of $2.67 \mathrm{GHz}$ speed and $12.0 \mathrm{~GB}$ RAM. We considered two topologies, Topology 1 and Topology 2. 


\subsubsection{Topology 1}

Topology 1 (Figure 5.1) is a static grid topology of 16 nodes. The grid is of size $3 D \times 3 D$, and the transmission range of the nodes is $R$, with $R=D \sqrt{2}$. It follows that, for example, node 10 has 8 neighbors: nodes $5,6,7,9,11,13,14$, and 15 . Likewise, node 1 has three neighbors: nodes 2,5 , and 6 . We set $M$ such that any node can simultaneously communicate with all its neighbors. Given our topology, the maximum number of neighbors that a node can have is 8 ; therefore we set $M=8$ when using MBAs. Let us consider eight traffic flows, flow 1 through flow 8, as follows: $1 \longrightarrow 15,3 \longrightarrow 13,5 \longrightarrow 12,9 \longrightarrow 8,15 \longrightarrow 2,13 \longrightarrow 4,12 \longrightarrow 1$, and $8 \longrightarrow 5$.

Table 5.2 presents the optimal link scheduling of the network as found by the solver. We can obtain from the table that the optimal average end-to-end delay is 3.25 time slots. 6 flows are completed in 3 time slots and 2 flows are completed in 4 time slots. All the 8 routes chosen are also the shortest (3 hops). Note that, given the regularity of our grid topology, each flow has many possibilities for its shortest route. It is worth also noting that the runtime to compute this solution (optimal link scheduling) for 16 nodes and 8 flows is 2 min 42 sec.

The optimal (delay-wise optimality) routes selected form a lot of star nodes in the network. We define star nodes as nodes that use their MPR capability at one time slot before using their MPT capability at the following time slot. With a star node, packets from distinct traffic flows travel two hops in two consecutive time slots, which is obviously a desirable effect for the minimization of the delay. From Table 5.2, we can see that one star node, namely node 6 , is formed between slot 1 and slot 2. In effect, flows 1 through 4 all send a packet to node 6 at slot 1 from different previous nodes (nodes 1, 3, 5, and 9 respectively) and all 4 packets leave node 6 for different next hops (nodes 11, 9, 7 and 3 respectively). We illustrate this star node in Figure 5.2 where, for clarity, we only show the first six flows at time slots 1 and 2. Later, three star nodes (nodes 11, 7, and 3) are formed between slot 2 and slot 3 . Finally, between slot 3 and slot 4 , one star node (node 6 ) is formed. 


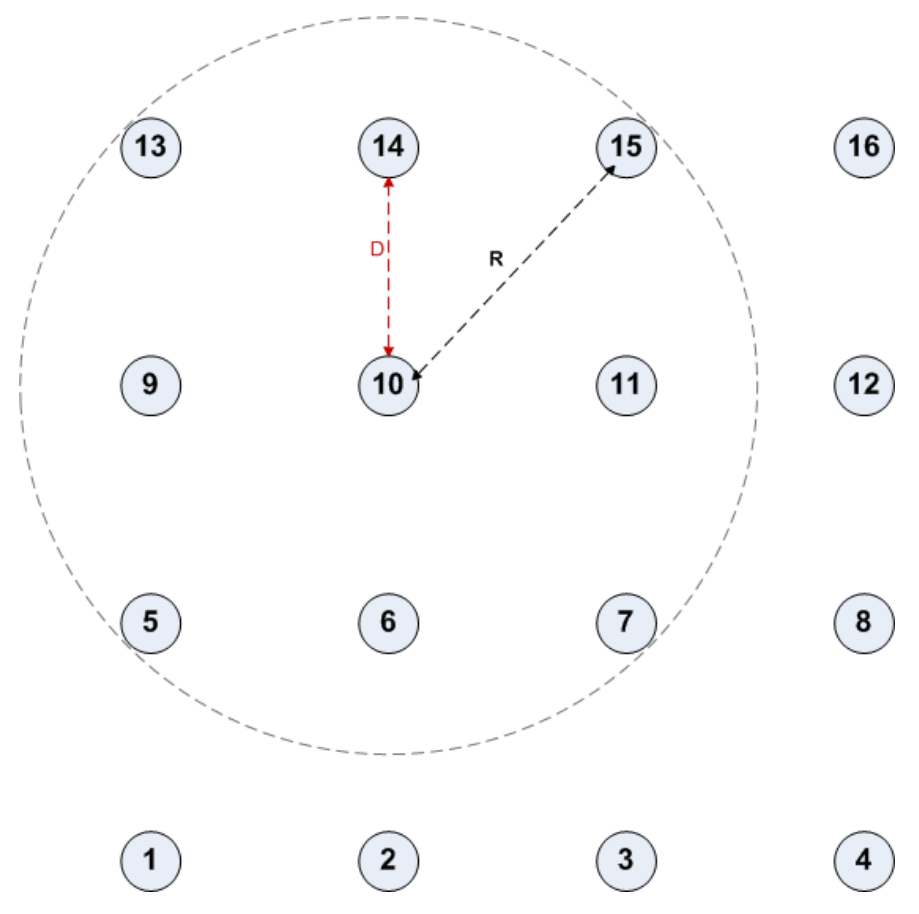

Figure 5.1: Topology 1

Table 5.2: Topology 1, Optimal Link Scheduling with 8 Flows

\begin{tabular}{lllll}
\hline Traffic flow & slot 1 & slot 2 & slot 3 & slot 4 \\
\hline \hline $1 \longrightarrow 15$ & $(1,6)$ & $(6,11)$ & $(11,15)$ & \\
$3 \longrightarrow 13$ & $(3,6)$ & $(6,9)$ & $(9,13)$ & \\
$5 \longrightarrow 12$ & $(5,6)$ & $(6,7)$ & $(7,12)$ & \\
$9 \longrightarrow 8$ & $(9,6)$ & $(6,3)$ & $(3,8)$ & \\
$15 \longrightarrow 2$ & $(15,12)$ & $(12,7)$ & $(7,2)$ & \\
$13 \longrightarrow 4$ & $(13,10)$ & $(10,7)$ & $(7,4)$ & \\
$12 \longrightarrow 1$ & & $(12,11)$ & $(11,6)$ & $(6,1)$ \\
$8 \longrightarrow 5$ & & $(8,3)$ & $(3,6)$ & $(6,5)$ \\
\hline
\end{tabular}

Two links fail to be scheduled at slot 1: link $(12,11)$ and link $(8,3)$ of flows 7 and 8 respectively. Link $(12,11)$ is not scheduled because node 12 (which would be the transmitter) is already at the receiving end of a scheduled link (link $(15,12)$ of flow 5$)$, and we know that a node cannot be receiving and transmitting at the same time (half-duplex constraint). Similarly, in the case of link $(8,3)$, node 3 that would be 
13

(14)

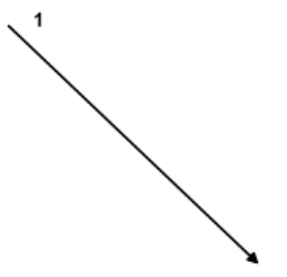

(9)

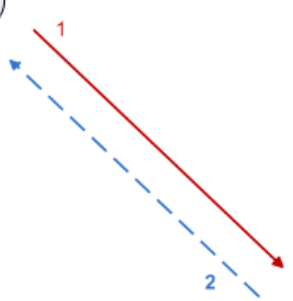

10

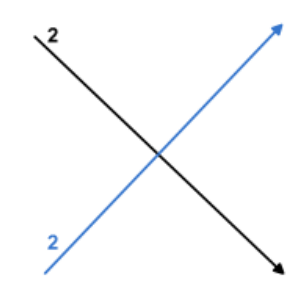

(15)

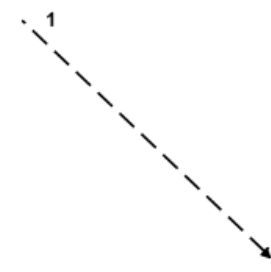

11

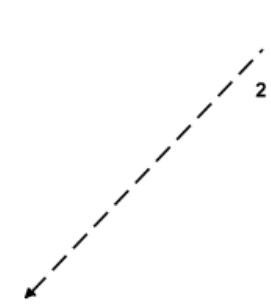

(16)

(12)

(8)

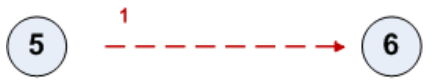

$\stackrel{2}{-}------\rightarrow 7$
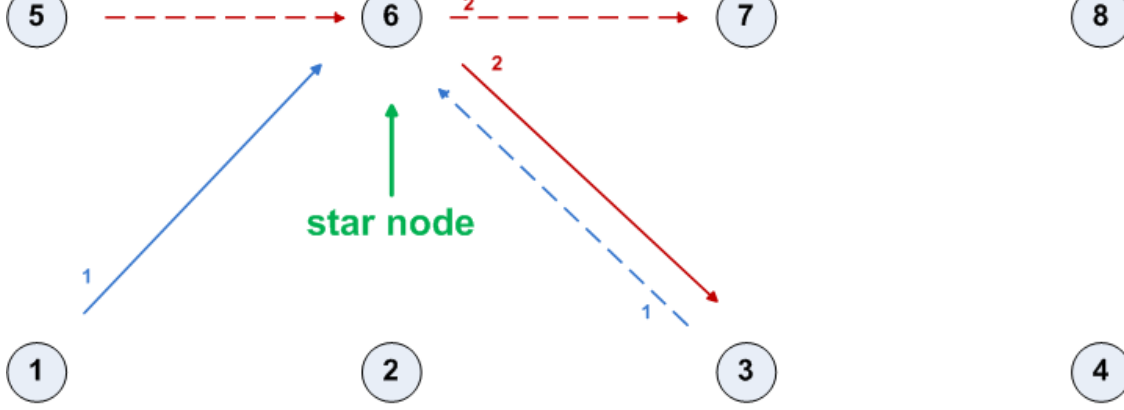

(3)

(4)

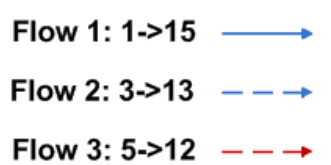

Flow 4: 9->8 $\longrightarrow$

Flow 5: 15->2 $--\rightarrow$

Flow $6: 13->10 \longrightarrow$

Figure 5.2: A Star Node

the receiver is already at the transmitting end of a scheduled link (link $(3,6)$ of flow 2$)$.

When MBAs are not used $(M=1)$, the performance (Table 5.3) degrades as follows. Only two flows (flow 4 and flow 7) are completed in 3 time slots, five flows are completed in 4 time slots, and one flow needs 6 time slots to complete. This gives an average of 4 time slots per flow, hence a degradation of 0.75 time slots compared to when MBAs are used. We also notice that three of the eight flows do not use their shortest path (3 hops), and use a longer (4 hops) path instead. This tells us that in some cases, using longer paths can improve the overall delay. With no MPT/MPR capability, the number of transmissions for the optimal scheduling is 27 , a $12.5 \%$ 
increase from the case with MPT/MPR (24 transmissions).

Table 5.3: Topology 1, Optimal Link Scheduling with 8 Flows, No MBA

\begin{tabular}{lllllll}
\hline Traffic flow & slot 1 & slot 2 & slot 3 & slot 4 & slot 5 & slot 6 \\
\hline \hline $1 \longrightarrow 15$ & & $(1,5)$ & $(5,10)$ & $(10,15)$ & & \\
$3 \longrightarrow 13$ & $(3,6)$ & $(6,9)$ & & $(9,13)$ & & \\
$5 \longrightarrow 12$ & $(5,10)$ & $(10,15)$ & $(15,16)$ & $(16,12)$ & & \\
$9 \longrightarrow 8$ & $(9,14)$ & $(14,11)$ & $(11,8)$ & & & \\
$15 \longrightarrow 2$ & $(15,16)$ & $(16,12)$ & $(12,7)$ & $(7,2)$ & & \\
$13 \longrightarrow 4$ & & & $(13,14)$ & $(14,11)$ & $(11,7)$ & $(7,4)$ \\
$12 \longrightarrow 1$ & $(12,7)$ & $(7,2)$ & $(2,1)$ & & & \\
$8 \longrightarrow 5$ & & $(8,3)$ & $(3,6)$ & $(6,5)$ & & \\
\hline
\end{tabular}

In any scenario, the scheduling of a given link is prohibited at a given time slot for two reasons: the non-availability of the MPT/MPR capability and/or the half-duplex constraint. For instance, link $(13,14)$ from flow 6 is not scheduled at slot 1 because of the non-availability of the MPT/MPR capability. In fact, node 14 is already receiving a packet from node 9 as part of flow 4 since link $(9,14)$ of flow 4 is scheduled. Link $(13,14)$ is not scheduled at slot 2 either. At this slot, it is the half-duplex constraint that prevents the scheduling since node 14 is already at the transmitting end of link $(14,11)$ of flow 4.

\subsubsection{Topology 2}

Topology 2 (Figure 5.3) is obtained by constraining/altering Topology 1 significantly. Nodes 5, 8, 9, and 12 are suppressed, resulting in a 12-node topology. In this network we have 6 traffic flows, flow 1 through flow 6 , as follows: $1 \longrightarrow 15,3 \longrightarrow 13$, $2 \longrightarrow 14,16 \longrightarrow 1,13 \longrightarrow 4$, and $14 \longrightarrow 3$. 
(13)

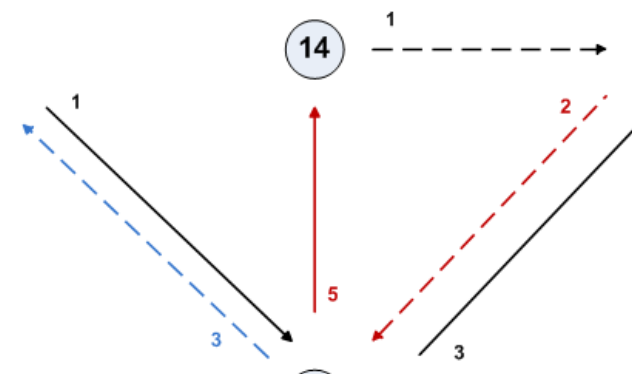

(15)

(10)
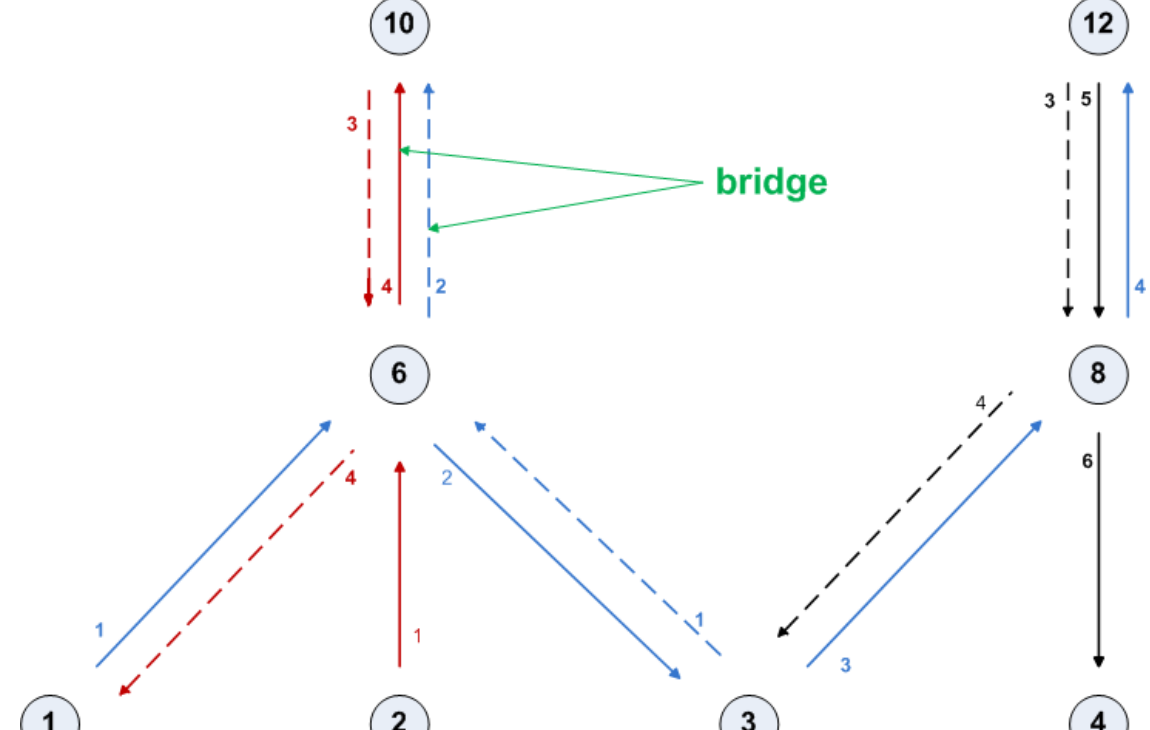

(6)

(1)

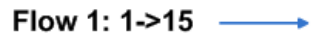

Flow 2: 3->13 $--\rightarrow$

Flow 3: 2->14

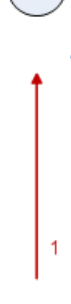

(2)

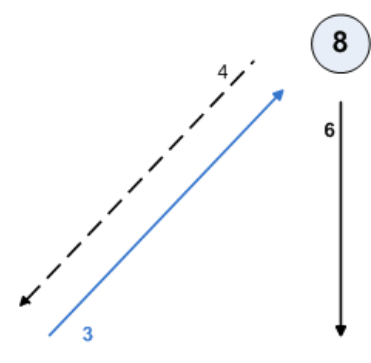

(3)
Flow 4: 16->1 $--\rightarrow$

Flow $5:$ 13->4 $\longrightarrow$

Flow 6: 14->3 - $\rightarrow$

Figure 5.3: Topology 2

The optimal link scheduling for Topology 2 is presented in Table 5.4 and Figure 5.3. It can be observed that the optimal end-to-end delay is 4.5 time slots per flow. Flow 5 is completed in 6 time slots, flows 1 and 3 are completed in 5 time slots, flows 4 and 6 are completed in 4 time slots, and flow 2 takes 3 time slots; thus the average of 4.5 time slots per flow. The runtime to compute this solution for 12 nodes and 6 flows is 1 min 01 sec.

From Table 5.4, we can see that five star nodes are formed as well as one bridge. We define a bridge as a link that routes two or more different flows that have arrived at a given node at the same time slot. Node 6 is a star node for flows 1 and 2 
Table 5.4: Topology 2, Optimal Link Scheduling with 6 Flows

\begin{tabular}{lllllll}
\hline Traffic flow & slot 1 & slot 2 & slot 3 & slot 4 & slot 5 & slot 6 \\
\hline \hline $1 \longrightarrow 15$ & $(1,6)$ & $(6,3)$ & $(3,8)$ & $(8,12)$ & $(12,15)$ & \\
$3 \longrightarrow 13$ & $(3,6)$ & $(6,10)$ & $(10,13)$ & & & \\
$2 \longrightarrow 14$ & $(2,6)$ & & & $(6,10)$ & $(10,14)$ & \\
$16 \longrightarrow 1$ & $(16,15)$ & $(15,10)$ & $(10,6)$ & $(6,1)$ & & \\
$13 \longrightarrow 4$ & $(13,10)$ & & $(10,15)$ & $(15,12)$ & $(12,8)$ & $(8,4)$ \\
$14 \longrightarrow 3$ & $(14,15)$ & $(15,12)$ & $(12,8)$ & $(8,3)$ & & \\
\hline
\end{tabular}

between slots 1 and 2. However, node 6 also forms a bridge for flows 2 and 3 at slot 2. In effect, packets from flows 2 and 3 both arrive at node 6 at slot 1 , and both packets (from two different flows) are scheduled to leave node 6 using the same link $(6,10)$, thus the same antenna beam: this is a bridge. Consequently, one of the flows has to wait (queuing delay) until a subsequent slot. As we can see, link $(6,10)$ of flow 3 is rescheduled for slot 4 . It cannot be rescheduled for slot 2 because it is already scheduled for flow 2, and it cannot be rescheduled at slot 3 either because of the half-duplex constraint (node 6 that is supposed to be the transmitter is already at the receiving end of link $(10,6)$ of flow 4$)$. Table 5.4 gives us the optimal solution, which means that this bridge could not be avoided. In comparison, we note that we do not have any bridge formed in the optimal scheduling of Topology 1 (See Table 5.2). In effect, many nodes in Topology 1 receive multiple flows at once at given time slots (node 6 at slot 1, nodes 11, 7, 3 at slot 2, and node 6 again at slot 3) and therefore could have formed bridges in subsequent time slots but did not. In brief, we see that bridges are absent in the optimal scheduling of Topology 1, and we have only a single one in the optimal scheduling of Topology 2. This suggests that bridges tend to be avoided, and are only kept when they are unavoidable and/or contribute to the optimal solution.

Back to the optimal scheduling of Topology 2, we can see that node 15 is a star node for flows 4 and 6 between slots 1 and 2. Node 10 is a star node for flows 2 and 4 between slots 2 and 3 . In addition, node 10 takes the slot 3 opportunity to also service flow 5 whose packet was received earlier at slot 1 . Therefore, by extension, node 10 is a star node for three flows (flows 2, 4, and 5). Node 8 is a star node 
for flows 1 and 6 between slots 3 and 4. Finally, node 12 is a star node for flows 1 and 5 between slots 4 and 5 . Note that link $(10,15)$ is not scheduled at slot 2 because of the half-duplex restriction for nodes 10 and 15 . Node 15 is already at the transmitting end of links $(15,10)$ and $(15,12)$ and node 10 is already at the receiving end of links $(6,10)$ and $(15,10)$.

Only half of the chosen routes are also the shortest (flows 2, 3, and 4). For flow 1 , a 5-hop route is chosen, marking a 2-hop increase from the shortest route. Similarly, for flow 5, the 5-hop route chosen is 1 hop longer than the shortest route between node 13 and node 4 (which is 4 hops with this new topology, unlike Topology 1). For flow 6 , the chosen route is 1 hop longer than the shortest route. We therefore observe that, even with MPT/MPR enabled, longer routes than the shortest are often preferred in order to attain the minimum E2E delay.

The number of transmissions performed is equal to the number of links scheduled. With the optimal link scheduling, we have a total of 24 transmissions.

When MBAs are not used $(M=1)$, the performance (Table 5.5) degrades as follows. Two flows are completed in 7 time slots, one flow is completed in 6 time slots, two flows are completed in 5 time slots, and one flow needs 3 time slots to complete. This gives an average of 5.5 time slots per flow. This is a degradation of 1 time slot per route compared to when MBAs are used. Here too, only half the routes chosen are also the shortest (flows 1, 2, and 3). We see that with this topology also, using longer paths does improve the overall delay. With no MPT/MPR capability, the number of transmissions for the optimal scheduling is 25 , a mere $4 \%$ increase from the case with MPT/MPR.

With Topology 2, unlike with Topology 1, there is only one possible shortest path for each flow; therefore we can also quantify the cost (in terms of delay) of using the shortest path here. The shortest paths are as follows:

Flow $1: 1 \rightarrow 6 \rightarrow 10 \rightarrow 15$

Flow $2: 3 \rightarrow 6 \rightarrow 10 \rightarrow 13$

Flow $3: 2 \rightarrow 6 \rightarrow 10 \rightarrow 14$

Flow $4: 16 \rightarrow 15 \rightarrow 10 \rightarrow 6 \rightarrow 1$ 
Flow $5: 13 \rightarrow 10 \rightarrow 6 \rightarrow 3 \rightarrow 4$

Flow $6: 14 \rightarrow 10 \rightarrow 6 \rightarrow 3$

Table 5.5: Topology 2, Optimal Link Scheduling with 6 Flows, No MBA

\begin{tabular}{llllllll}
\hline Traffic flow & slot 1 & slot 2 & slot 3 & slot 4 & slot 5 & slot 6 & slot 7 \\
\hline \hline $1 \longrightarrow 15$ & & & $(1,6)$ & $(6,10)$ & $(10,15)$ & & \\
$3 \longrightarrow 13$ & $(3,6)$ & $(6,10)$ & $(10,13)$ & & & & \\
$2 \longrightarrow 14$ & & & & & $(2,6)$ & $(6,10)$ & $(10,14)$ \\
$16 \longrightarrow 1$ & $(16,12)$ & $(12,8)$ & $(8,3)$ & $(3,2)$ & & $(2,1)$ & \\
$13 \longrightarrow 4$ & & $(13,14)$ & $(14,15)$ & $(15,16)$ & $(16,12)$ & $(12,8)$ & $(8,4)$ \\
$14 \longrightarrow 3$ & $(14,15)$ & $(15,16)$ & $(16,12)$ & $(12,8)$ & $(8,3)$ & & \\
\hline
\end{tabular}

The scheduling in Table 5.6 shows that if flows are restricted to their shortest route we have a considerable degradation in delay, even if MBAs are used. In effect, we can deduct from the table an average delay of 7 slots per flow, which is a degradation of 2.5 time slots from the optimal solution described above (4.5 time slots). Therefore, the latter is a $36 \%$ decrease in delay compared to the former. Moreover, using only shortest routes with MBAs also shows a 1.5 slot degradation from the optimal solution without MBAs. We therefore observe that limiting routes to the shortest paths, even while using MBAs, is detrimental to the delay to the point that having a "smarter" choice of paths (some paths being longer than the shortest) without MBAs is even better. The number of transmissions here is equal to 20 , a $16 \%$ decrease from the optimal scheduling presented earlier. Unfortunately, this reduction in overhead (measured as the number of transmissions) comes at the expense of a substantial increase in E2E delay.

If we use the shortest path and no MBAs, Table 5.7 shows a prohibitive end-to-end delay of $11.3 \overline{3}$ slots per flow on average. This is a degradation of $5.8 \overline{3}$ time slots compared to a smarter choice of paths without MBAs, and an even worse degradation of $6.8 \overline{3}$ time slots compared to a smarter choice of paths with MBAs. The number of transmissions is also 20 as in the case of MBAs; which is not 
Table 5.6: Topology 2, Link Scheduling with 6 Flows, Shortest Path

\begin{tabular}{|c|c|c|c|c|c|c|c|c|c|c|}
\hline Flow & slot 1 & slot 2 & slot 3 & slot 4 & slot 5 & slot 6 & slot 7 & slot 8 & slot 9 & slot 10 \\
\hline $1 \rightarrow 15$ & $(1,6)$ & $(6,10)$ & & & $(10,15)$ & & & & & \\
\hline $3 \rightarrow 13$ & $(3,6)$ & & $(6,10)$ & & $(10,13)$ & & & & & \\
\hline $2 \rightarrow 14$ & $(2,6)$ & & & $(6,10)$ & $(10,14)$ & & & & & \\
\hline $16 \rightarrow 1$ & $(16,15)$ & $(15,10)$ & & & & & $(10,6)$ & $(6,1)$ & & \\
\hline $13 \rightarrow 4$ & $(13,10)$ & & & & $(10,6)$ & & & $(6,3)$ & & $(3,4)$ \\
\hline $14 \rightarrow 3$ & $(14,10)$ & & & & & $(10,6)$ & & & $(6,3)$ & \\
\hline
\end{tabular}

Table 5.7: Topology 2, Link Scheduling with 6 Flows, Shortest Path, No MBA

\begin{tabular}{|c|c|c|c|c|c|c|c|c|c|c|c|c|c|}
\hline Flow & slot 1 & slot 2 & slot 3 & slot 4 & slot 5 & slot 6 & slot 7 & slot 8 & slot 9 & slot 10 & slot 11 & slot 12 & slot 13 \\
\hline $1 \rightarrow 15$ & $(1,6)$ & & & $(6,10)$ & & & & & & $(10,15)$ & & & \\
\hline $3 \rightarrow 13$ & & $(3,6)$ & & & $(6,10)$ & & & & & & $(10,13)$ & & \\
\hline $2 \rightarrow 14$ & & & $(2,6)$ & & & $(6,10)$ & & & & & & $(10,14)$ & \\
\hline $16 \rightarrow 1$ & $(16,15)$ & & $(15,10)$ & & & & $(10,6)$ & & & $(6,1)$ & & & \\
\hline $13 \rightarrow 4$ & $(13,10)$ & & & & & & & $(10,6)$ & & & $(6,3)$ & & $(3,4)$ \\
\hline $14 \rightarrow 3$ & & $(14,10)$ & & & & & & & $(10,6)$ & & & $(6,3)$ & \\
\hline
\end{tabular}

surprising since the shortest path is used in both cases and the length of the paths determines the number of transmissions.

The takeaway from the above discussion on Topology 1 and Topology 2 can be summarized in six points:

1. In the presence of the MPT/MPR capability, the optimal link scheduling has a proclivity of forming star nodes as much as possible in order to forward packets from many different flows during the same two time slots.

2. In the presence of the MPT/MPR capability, the optimal link scheduling tends to avoid bridges in order to avoid queuing delays. However, some bridges are simply unavoidable and/or are necessary for the optimal result, as we end up getting enough benefit from the additional stars that they allow to be created.

3. In the presence of the MPT/MPR capability, longer routes are often preferred in order to minimize the delay. This happens when the overall waiting/rescheduling delay is greater than the overall delay of employing longer routes. 
4. Even with MPT/MPR disabled, longer routes are often preferred in order to attain the optimal end-to-end delay.

5. A smart use of the MPT/MPR capability considerably reduces the end-to-end delay. This smart use means: the promotion of star nodes and the elimination of bridges as much as possible (not necessarily all of them); resulting in a choice of routes that are not necessarily the shortest in terms of hops.

6. With optimal scheduling, the reduction in delay comes at the expense of an increase in overhead measured in terms of the total number of transmissions performed.

In the next section, we refine our model by adding antenna beams to it. This addition shall make the model more realistic and applicable to MANETs. Assuming that each link has a dedicated beam (only one neighbor at most per beam), as we have done thus far, is only applicable when the topology is carefully chosen to enforce that; which is far from being the case when nodes move randomly.

\subsection{Considering Antenna Beams}

In the model presented earlier in Section 5.2.2, we assumed idealistic conditions whereby each link to a neighbor falls inside a dedicated beam. No two neighbors or more could be within the same beam. In this section, we make the model more realistic by introducing the notion of beams. The optimization model is now as follows.

\subsubsection{Modified Optimization Model}

The general description (of the model) presented in Section 5.2 still holds here, except for the assumption about each link having a dedicated antenna beam. We still represent the network with a directed connectivity graph $G(V, E)$. However, $G(V, E)$ is no longer a direct input. Instead, a matrix $A B$ containing the position and the direction angle of all the nodes is received as an input. $G(V, E)$ is then constructed based on the information contained in $A B$ and considering the beams of each node. As already noted, $G(V, E)$ is a directed connectivity graph where $V$ represents the set of nodes (or vertices) in the network, and $E$ is the set of directed links (or edges). Now, we 
represent a link with an additional information, the beam identifier $m$ on which the sender $i$ reaches the receiver $j$. We represent a link as $(i, j, m)$. Basically, if node $j$ is within the transmission range of node $i$ on the latter's $m^{\text {th }}$ beam, then links $(i, j, m)$ and $(j, i, n)$ are members of $E$. Note that $n$ is the beam on which node $j$ (the receiver) would reach node $i$. In the outputs, the selected route for each flow, presented as a sequence of links, now also shows the antenna beam identifier of the sender of each link.

Table 5.8: Notation for MBA-DM-2

\begin{tabular}{ll}
\hline$\alpha_{i j m}^{f k}$ & Scheduling status of link $(i, j)$ of flow $f$, on beam $m$ of \\
& node $i$ at time slot $k$ \\
$\alpha_{i d_{f} m}^{f k}$ & Scheduling status of link $\left(i, d_{f}\right)$ of flow $f$, , on beam $m$ of \\
& node $i$ at time slot $k$ \\
$m \leq M \mid(i, j, m) \in E$ & Beam $m$ on which node $i$ reaches node $j$ \\
\hline
\end{tabular}

In order to include the antenna beams in our optimization problem, we enhance the decision variable so that it includes the antenna beam number (identifier) $m$ on which the sender reaches the receiver. Therefore, the decision variable is now $\alpha_{i j m}^{f k}$ which tells us whether or not link $(i, j, m)$ of flow $f$ is scheduled at time slot $k$. $\alpha_{i j m}^{f k} \in\{0,1\}$. We designate this modified optimization problem as MBA-DM-2 (Delay Minimization with MBAs, Version 2). Table 5.8 explains some new symbols not yet explained in Table 5.1. MBA-DM-2 is formulated as follows:

\section{MBA-DM-2:}

Minimize $\sum_{f \in F} \sum_{k \in T} \sum_{i \in V} \sum_{m \leq M} \alpha_{i d_{f} m}^{f k} \cdot k$

subject to the following 7 constraints:

1. Flow Circulation Constraints

$$
\sum_{k \in T} \sum_{j \in V} \sum_{m \leq M \mid\left(s_{f}, j, m\right) \in E} \alpha_{s_{f} j m}^{f k}=1 \quad \forall f \in F
$$




$$
\begin{array}{ll}
\sum_{k \in T} \sum_{i \in V} \sum_{m \leq M \mid\left(i, d_{f}, m\right) \in E} \alpha_{i d_{f} m}^{f k}=1 & \forall f \in F \\
\alpha_{i j m}^{f k}-\sum_{t=1}^{k-1} \sum_{l \in V} \sum_{n \mid(l, i, n) \in E} \alpha_{l i n}^{f t} \leq 0 \quad & \forall f \in F, \forall k \in T, \forall i \in V-\left\{s_{f}\right\}, \\
& \forall j \in V, \forall m \leq M \mid(i, j, m) \in E
\end{array}
$$

2. Flow Consistency Constraints

$$
\begin{aligned}
& \sum_{l \in V} \sum_{m \leq M \mid(i, l, m) \in E} \alpha_{i l m}^{f k} \leq 1 \quad \forall f \in F, \forall k \in T, \forall i \in V \\
& \sum_{l \in V} \sum_{m \leq M \mid(l, j, m) \in E} \alpha_{l j m}^{f k} \leq 1 \quad \forall f \in F, \forall k \in T, \forall j \in V \\
& \sum_{k \in T} \alpha_{i j m}^{f k} \leq 1 \quad \forall f \in F, \forall(i, j, m) \in E
\end{aligned}
$$

3. Link Occupancy Constraint

$$
\sum_{f \in F} \alpha_{i j m}^{f k} \leq 1 \quad \forall k \in T, \forall(i, j, m) \in E
$$

4. Half-duplex Constraint

$$
\sum_{f \in F} \sum_{m \leq M \mid(i, j, m) \in E} \sum_{n \leq M \mid(j, l, n) \in E}\left(\alpha_{i j m}^{f k}+\alpha_{j l n}^{f k}\right) \leq 1 \quad \forall k \in T, \forall i, j, l \in V
$$

5. MPT Capability Constraint

$$
\sum_{f \in F} \sum_{l \in V} \sum_{m \leq M \mid(i, l, m) \in E} \alpha_{i l m}^{f k} \leq M \quad \forall k \in T, \forall i \in V
$$


6. MPR Capability Constraint

$$
\sum_{f \in F} \sum_{l \in V} \sum_{m \leq M \mid(l, j, m) \in E} \alpha_{l j m}^{f k} \leq M \quad \forall k \in T, \forall j \in V
$$

7. Beam Occupancy Constraints

$$
\begin{aligned}
& \sum_{f \in F} \sum_{l \in V \mid(i, l, m) \in E} \alpha_{i l m}^{f k} \leq 1 \quad \forall i \in V, \forall k \in T, \forall m \leq M \\
& \sum_{f \in F} \sum_{l \in V \mid(j, l, m) \in E} \sum_{n \leq M \mid(l, j, n) \in E} \alpha_{l j n}^{f k} \leq 1 \quad \forall j \in V, \forall k \in T, \forall m \leq M
\end{aligned}
$$

The objective function (5.12) and the first 6 constraints have already been explained in Section 5.2.2. Here we simply adjusted them in order to include the beam index/variable. However, we did add an additional (a $7^{\text {th }}$ ) constraint, the Beam Occupancy Constraint, that is explained as follows:

- Constraint 5.23: the same beam cannot service multiple outgoing links at a given time slot.

- Constraint 5.24: the same beam cannot service multiple incoming links at a given time slot. Note that here, compared to 5.23, we have one additional (a third one) summation pertaining to the beam number $n$. These are the beam numbers appearing in the decision variable of the constraint as well. However, there is a first beam number $m$ that "conditions" the $l$ nodes (the senders) in the second summation. The reason is as follows. The second summation considers all the neighboring nodes (senders) that are within a given beam $m$ of a given receiver $j$. These sender nodes, on their part, reach the receiver $j$ with beam numbers $n$ that are unrelated to $m$. The distinction is important: beam number $m$ pertains to the receiver whereas beam number $n$ pertains to the sender. The goal of the constraint is to disallow simultaneous senders reaching a common receiver when these two senders are within the same beam of the common receiver. 
Note that the Beam Occupancy Constraint is different from the Link Occupancy Constraint. However, these two constraints are complementary. In effect, the Link Occupancy Constraint ensures that only one flow is permitted on a given link (a pair of nodes) at a time. The Beam Occupancy Constraint, in its turn, ensures that only one link is permitted on a given beam at a time, since multiple links can indeed fall within the same beam.

\subsubsection{Modified-Model Results and Analysis}

With the foregoing modified optimization model, let us consider Topology 2 (Figure 5.3) once again. We assume that all the nodes have the same direction of movement of $-43^{\circ}$ (the reference of $0^{\circ}$ being the horizontal line that points to the East). This direction is chosen arbitrarily. Recall from Section 2.3 that the beams are numbered in ascending order counterclockwise, with the front (or direction of movement) being the relative reference line (Figure 2.1). The 6 traffic flows, flow 1 through flow 6 , are the same as previously: $1 \longrightarrow 15,3 \longrightarrow 13,2 \longrightarrow 14,16 \longrightarrow 1$, $13 \longrightarrow 4$, and $14 \longrightarrow 3$.

In order to see how the beamwidth affects the route selection and the optimal solution, we consider three different beamwidths: $45^{\circ}, 60^{\circ}$, and $120^{\circ}$. In effect, with our topology and direction of movement of the nodes, with a $45^{\circ}$ beamwidth, each beam of any node is expected to reach only one neighbor at most. With a $60^{\circ}$ beamwidth, some beams are able to reach up to two neighbors. Finally, with a $120^{\circ}$ beamwidth, some beams are able to reach up to three neighbors. In the following results tables, we adopt a notation convention whereby $\left(X^{(m)}, Y\right)$ indicates that the link between nodes $X$ and $Y$ is scheduled, and that node $X$ actually uses its beam number $m$ to reach node $Y$.

The optimal link scheduling with a $45^{\circ}$ beamwidth is presented in Table 5.9. It can be observed that the optimal end-to-end delay is 4.5 slots per flow. Flow 5 is completed in 6 slots, flows 1 and 3 are completed in 5 slots, flows 4 and 6 are completed in 4 slots, and flow 2 takes 3 slots; thus the average of 4.5 slots per flow. The runtime to compute this solution is 7 min 08 sec. As we can see, this optimal

link scheduling is identical (same star nodes, same bridge, same routes, etc.) to the scheduling of the previous model presented in Table 5.4. This comes as no surprise 
since, as already mentioned, a $45^{\circ}$ beamwidth ensures that each link gets its own (dedicated) beam; which was the assumption in the previous model. This result simply shows that the new model (adding beam considerations) works well and is consistent with the previous one. This is a validation test. However, as we can also see, it takes significantly more time $(7 \mathrm{~min} 08 \mathrm{sec}$ as opposed to $1 \mathrm{~min} 01 \mathrm{sec}$ ) for the solver to find the optimal solution with the new model that is more realistic for MBA-MANETs. As already mentioned, the same stars and bridge from Table 5.4 are present in Table 5.9. However, in the latter, the specific beams that are used in transmissions are indicated. In fact, with a beamwidth of $45^{\circ}$, each antenna has 8 beams. We can see that, for example, node 6 (that forms a star for flows 1 and 2 between slots 1 and 2) uses its beam 8 to forward a flow-1 packet to node 3 and its beam 3 to forward a flow-2 packet to node 10. At slot 1, node 6 had received both packets on two different beams as well, beams 6 and 8 respectively. These reception beams are not explicitly shown as such in the table. Rather, we see them (indirectly) from transmissions from node 6 to the nodes concerned (node 1 and node 3) somewhere else in the table. In effect, if node 6 sends to a given node using a certain beam, then it receives from that same beam when receiving from the given node as well, since we compute the optimal solution based on a static/snapshot topology.

Table 5.9: Topology 2, Optimal Link Scheduling with $45^{\circ}$ Beamwidth

\begin{tabular}{lllllll}
\hline Traffic flow & slot 1 & slot 2 & slot 3 & slot 4 & slot 5 & slot 6 \\
\hline \hline $1 \longrightarrow 15$ & $\left(1^{(2)}, 6\right)$ & $\left(6^{(8)}, 3\right)$ & $\left(3^{(2)}, 8\right)$ & $\left(8^{(3)}, 12\right)$ & $\left(12^{(4)}, 15\right)$ & \\
$3 \longrightarrow 13$ & $\left(3^{(4)}, 6\right)$ & $\left(6^{(3)}, 10\right)$ & $\left(10^{(4)}, 13\right)$ & & & \\
$2 \longrightarrow 14$ & $\left(2^{(3)}, 6\right)$ & & & $\left(6^{(3)}, 10\right)$ & $\left(10^{(3)}, 14\right)$ & \\
$16 \longrightarrow 1$ & $\left(16^{(5)}, 15\right)$ & $\left(15^{(6)}, 10\right)$ & $\left(10^{(7)}, 6\right)$ & $\left(6^{(6)}, 1\right)$ & & \\
$13 \longrightarrow 4$ & $\left(13^{(8)}, 10\right)$ & & $\left(10^{(2)}, 15\right)$ & $\left(15^{(8)}, 12\right)$ & $\left(12^{(7)}, 8\right)$ & $\left(8^{(7)}, 4\right)$ \\
$14 \longrightarrow 3$ & $\left(14^{(1)}, 15\right)$ & $\left(15^{(8)}, 12\right)$ & $\left(12^{(7)}, 8\right)$ & $\left(8^{(6)}, 3\right)$ & & \\
\hline
\end{tabular}

The optimal link scheduling with a $60^{\circ}$ beamwidth is presented in Table 5.10. It can be observed that the optimal end-to-end delay is still 4.5 slots per flow despite the increase of the beamwidth. However, the routes have been scheduled slightly 
differently. For instance, with the $60^{\circ}$ beamwidth, nodes 2 and 3 now find themselves located within the same beam of node 6 (its beam number 6 ). Therefore, links $(2,6)$ and $(3,6)$ can no longer be scheduled simultaneously, as was the case with a $45^{\circ}$ beamwidth in Table 5.9. Instead, link $(3,6)$ now has to wait, and is not scheduled until time slot 3 . This is a case of an unavoidable bridge occasioned by a receiving beam in common. Flow 2 now completes in five time slots whereas flow 3 does so in just three. It was the opposite in the $45^{\circ}$ beamwidth scheduling. Recall that, in the case of the $45^{\circ}$ beamwidth, the bridge situation was occasioned by a link in common (link $(6,10))$ at slot 2 . In brief, having a larger beamwidth has caused the appearance of a beam-in-common bridge, which has prompted the rescheduling of flows 2 and 3 for optimal delay. Note that there are two types of bridges: link-in-common bridges and beam-in-common bridges. Link-in-common bridges involve a common link (pair of nodes) that is to serve many flows at a given time slot. Beam-in-common bridges involve a common beam that is to serve many flows at a given time slot, even though this service uses distinct links (the links do share a sender or a receiver). The larger the beamwidth the more there is a potential to encounter beam-in-common bridges. The runtime to compute this solution with a $60^{\circ}$ beamwidth is 5 min 31 sec, considerably lower than with a narrower beamwidth of $45^{\circ}$.

Table 5.10: Topology 2, Optimal Link Scheduling with $60^{\circ}$ Beamwidth

\begin{tabular}{lllllll}
\hline Traffic flow & slot 1 & slot 2 & slot 3 & slot 4 & slot 5 & slot 6 \\
\hline \hline $1 \longrightarrow 15$ & $\left(1^{(2)}, 6\right)$ & $\left(6^{(6)}, 3\right)$ & $\left(3^{(2)}, 8\right)$ & $\left(8^{(3)}, 12\right)$ & $\left(12^{(3)}, 15\right)$ & \\
$3 \longrightarrow 13$ & & & $\left(3^{(3)}, 6\right)$ & $\left(6^{(3)}, 10\right)$ & $\left(10^{(3)}, 13\right)$ & \\
$2 \longrightarrow 14$ & $\left(2^{(3)}, 6\right)$ & $\left(6^{(3)}, 10\right)$ & $\left(10^{(3)}, 14\right)$ & & & \\
$16 \longrightarrow 1$ & $\left(16^{(4)}, 15\right)$ & $\left(15^{(5)}, 10\right)$ & $\left(10^{(6)}, 6\right)$ & $\left(6^{(5)}, 1\right)$ & & \\
$13 \longrightarrow 4$ & $\left(13^{(6)}, 10\right)$ & & $\left(10^{(2)}, 15\right)$ & $\left(15^{(6)}, 12\right)$ & $\left(12^{(6)}, 8\right)$ & $\left(8^{(6)}, 4\right)$ \\
$14 \longrightarrow 3$ & $\left(14^{(1)}, 15\right)$ & $\left(15^{(6)}, 12\right)$ & $\left(12^{(6)}, 8\right)$ & $\left(8^{(5)}, 3\right)$ & & \\
\hline
\end{tabular}

The optimal link scheduling with a $120^{\circ}$ beamwidth is presented in Table 5.11. It can be observed that the optimal end-to-end delay has now increased to $4.83 \overline{3}$ time slots per flow with the increase of the beamwidth. In fact, flows 1 and 4 are completed in 6 time slots, flow 2 is completed in 3 time slots, flows 3 and 5 are 
completed in 5 time slots, and flow 6 takes 4 time slots; hence the average of $4.83 \overline{3}$ time slots per flow. With the increase in beamwidth, we now have, for example, nodes 1, 2, and 3 all located within the same beam (beam 3) of node 6 . Therefore, only one of these nodes can be scheduled to transmit to node 6 at a time. In this case, as we can see, a mere rescheduling is no longer sufficient in order to attain the optimal delay. A re-routing of some flows is now also necessary. In effect, flows 1 , 5, and 6 are re-routed in the first two time slots. Finally, the runtime to compute this $120^{\circ}$-beamwidth solution is $3 \mathrm{~min} 31 \mathrm{sec}$, which is significantly lower than with narrower beamwidths.

Table 5.11: Topology 2, Optimal Link Scheduling with $120^{\circ}$ Beamwidth

\begin{tabular}{lllllll}
\hline Traffic flow & slot 1 & slot 2 & slot 3 & slot 4 & slot 5 & slot 6 \\
\hline \hline $1 \longrightarrow 15$ & $\left(1^{(1)}, 2\right)$ & $\left(2^{(1)}, 3\right)$ & $\left(3^{(1)}, 8\right)$ & & $\left(8^{(2)}, 12\right)$ & $\left(12^{(2)}, 15\right)$ \\
$3 \longrightarrow 13$ & $\left(3^{(2)}, 6\right)$ & $\left(6^{(2)}, 10\right)$ & $\left(10^{(2)}, 13\right)$ & & & \\
$2 \longrightarrow 14$ & & & $\left(2^{(2)}, 6\right)$ & $\left(6^{(2)}, 10\right)$ & $\left(10^{(2)}, 14\right)$ & \\
$16 \longrightarrow 1$ & $\left(16^{(2)}, 15\right)$ & & & $\left(15^{(3)}, 10\right)$ & $\left(10^{(3)}, 6\right)$ & $\left(6^{(3)}, 1\right)$ \\
$13 \longrightarrow 4$ & $\left(13^{(1)}, 14\right)$ & $\left(14^{(1)}, 15\right)$ & $\left(15^{(3)}, 12\right)$ & $\left(12^{(3)}, 8\right)$ & $\left(8^{(3)}, 4\right)$ & \\
$14 \longrightarrow 3$ & & $\left(14^{(3)}, 10\right)$ & $\left(10^{(3)}, 6\right)$ & $\left(6^{(3)}, 3\right)$ & & \\
\hline
\end{tabular}

The takeaway from solving this modified and "beam-aware" model can be summarized in the following three points:

1. The discussion and the conclusions on the "beam-less" model also hold for the "beam-aware" model. This comes as no surprise inasmuch as the "beamless" model actually assumes that beams are set up such that there is only one possible neighbor (or link) at most per beam. In brief, the beam-aware model is a generalization of the beam-less model. In fact, the beam-aware model becomes the beam-less model if the beamwidth is set to a small-enough value (so as to accommodate a single link only).

2. The trend is that narrower beams lead to reduced E2E latency. But in a mobile scenario, narrower beams make it more difficult to track neighboring nodes at 
the MAC layer. So to employ MBAs in MANETs, the right balance has to be found/determined.

3. Computing time increases with narrower beamwidths (inherently larger number of beams), as we now have more variables and equations/constraints to process.

\subsection{Summary}

In this chapter, basing our analysis on static deterministic topologies with multi-flow scenarios, we have shown that in order to exploit the full potential of MBAs for end-to-end delay reduction, the selection of routes needs to include other (other than route length) criteria such as the promotion of star nodes and the avoidance of bridges as much as possible. We have shown that the mere use of shortest routes, as it is the case in most existing routing protocols for ad hoc networks, results in relatively high delays because such paths usually result in the formation of bridges in multi-flow scenarios; and bridges incur queuing delays that add to the end-to-end delay. We formulated determining the best route selection (best link scheduling) as an optimization problem that we solved using linear programming. Our scenarios showed that the optimal link scheduling forms a lot of star nodes and avoids bridges unless there is a clear delay advantage in keeping them. Some bridges are necessary for the optimal result, as we end up getting enough benefit from the additional stars they allow to be created. We obtained a reduction in end-to-end delay compared to the shortest-path case. However, this considerable reduction in delay comes at the expense of an increase in overhead measured by the total number of transmissions.

The refinement of our optimization model with the introduction of beam considerations (beamforming, determination of the beam in which neighbors are located, etc.) has sustained the conclusions of the initial model. In addition, it has pointed out the impact of beamwidth on both end-to-end delay and computation time. In the next chapter, we discuss the design of a protocol that establishes routes in light of what we have learned here (promotion of star nodes and avoidance of bridges) in order to considerably reduce the E2E delay in ad hoc networks. This protocol might have to espouse a centralized SDN architecture, or a heuristic distributed design approach. 


\section{Chapter 6}

\section{The MBA-DRR Routing Protocol}

\subsection{Introduction}

The analysis in Chapter 5 shows the existence of a theoretical minimum E2E delay. For this minimum E2E delay to happen, two conditions need to be met: a) perfect synchronization between the nodes; and b) the presence of an omniscient central entity that schedules the links. The role of the central entity would be twofold. First it would synchronize all the nodes and control time slot assignments. Secondly, it would compute the optimal routes of all the flows and control which node gets to transmit at which time slot, using which beam. At first, all these conditions might seem to point to a Software-Defined Network (SDN) architecture. However, SDN is not practical at all in the context of many MANETs, as we see in Section 6.2. Nevertheless, we can still learn from Chapter 5 the characteristics of the routes that are constructed for the optimal solution, and try to replicate this as best as we can in a distributed heuristic algorithm. In effect, we learn that when the optimal solution is reached, the routes avoid bridges and promote star nodes as much as possible; a behavior that is conducive to delay reductions, but that, unfortunately, is not taken into account at all in traditional routing protocols.

Our goal is to design a routing protocol for static and mobile MBA ad hoc networks, a protocol that significantly reduces the E2E delay by fully exploiting the MPR/MPT potential of MBAs. The benefits of this protocol are expected to be applicable to other types of MBA-based ad hoc networks, both mobile and static. In light of the take-away from the delay optimization model, we propose to conduct route selection differently in order to avoid bridges and promote star nodes 
as much as possible. We do not expect our solution to be optimal, but at least it should result in a considerable improvement in terms of E2E delay, compared to the current shortest-path-based protocols and other protocols that focus on maximizing throughput with some MBA capability (such as the MPR capability alone).

\subsection{A Centralized Approach}

One way of achieving a centrally-controlled algorithm is to adopt an SDN approach. SDN $[99,100]$ is an emergent paradigm in computing and networking, which separates the control and data communication layers to simplify the network management and expedite system evolution. In SDN, the network intelligence is logically centralized in a software-based controller, and the nodes in the network will forward data packets based on the decisions made by this controller. There exist mechanisms for the controller to learn about the network topology. The SDN concept has gained some momentum lately in Wireless Distributed Networks (WDNs) [5, 90, 91, 99-105] such as VANETs, sensor networks, and mesh networks.

The problem with an SDN approach is that a central controller is needed. Almost all authors [5,90,91,99-105] that have explored this approach in VANETs and other WDNs have invariably assumed the presence of a central controller. Figure 6.1 shows a standard VANET SDN architecture. In FANETs for example, the idea of a central controller is not practical. Where would such central controller be located? Is it going to be mobile or fixed? Moreover, even if a central controller is hypothetically set fittingly, this controller will be responsible for computing the optimal paths for all the traffic flows based on its global view of the network. There is a problem that will then arise. The network topology of MANETs and FANETs is expected to change fairly frequently. Therefore, the topology that the optimization is based on at an instant $t_{1}$ is very likely to have changed significantly at a subsequent instant $t_{2}$ when all the nodes receive the link scheduling computed by the controller, especially since the optimization computing can take several minutes (see Chapter 5). And this computation time increases considerably with the size of the network; which does not bode well for scalability. In the several minutes separating $t_{1}$ and $t_{2}$, a lot will 


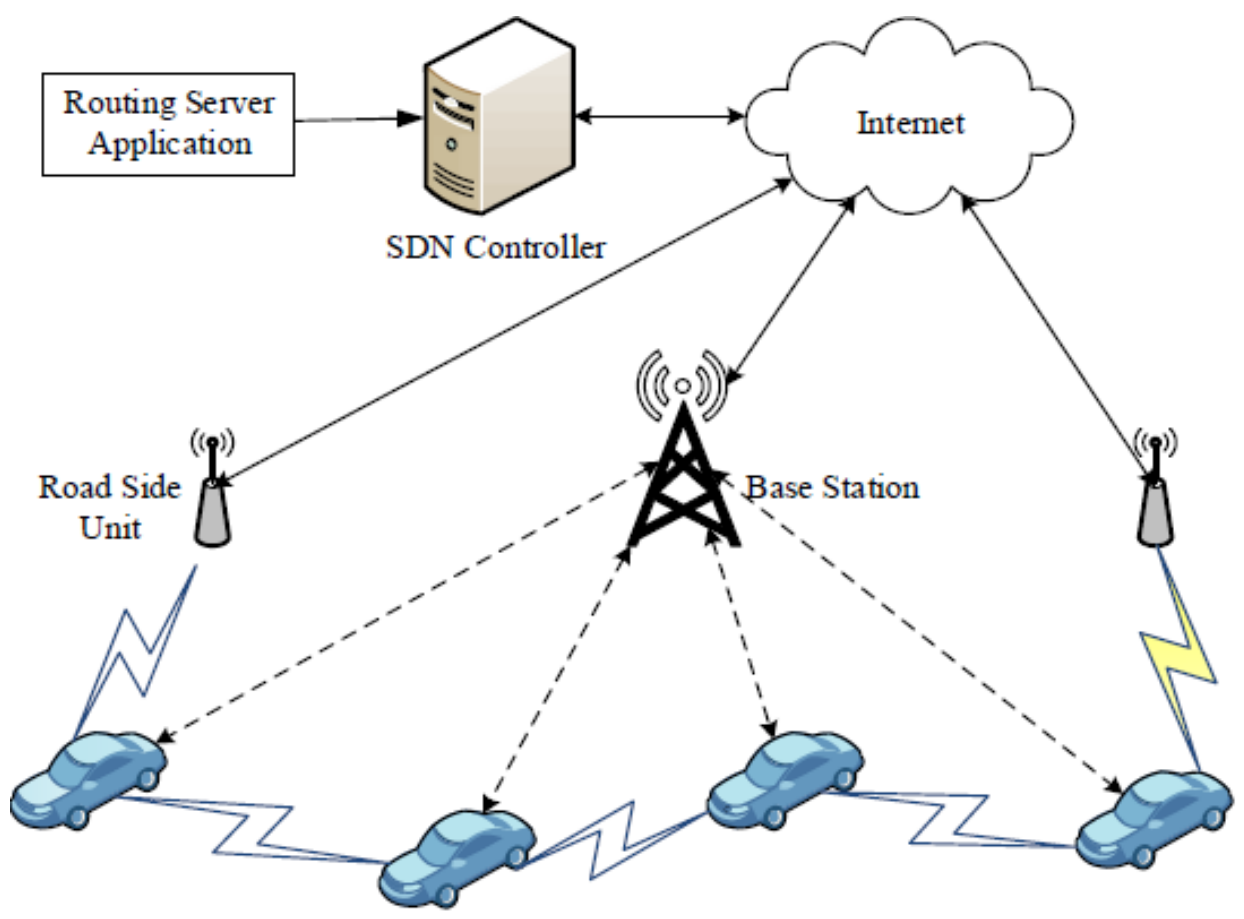

Figure 6.1: Standard SDN Architecture in VANETs [5] C2016 IEEE

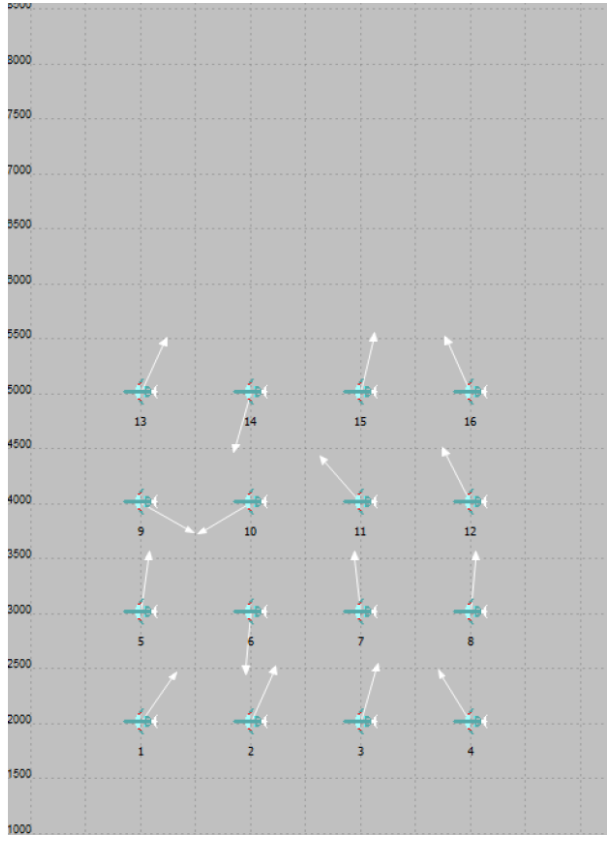

(a) Topology at 0 min $0 \mathrm{sec}$

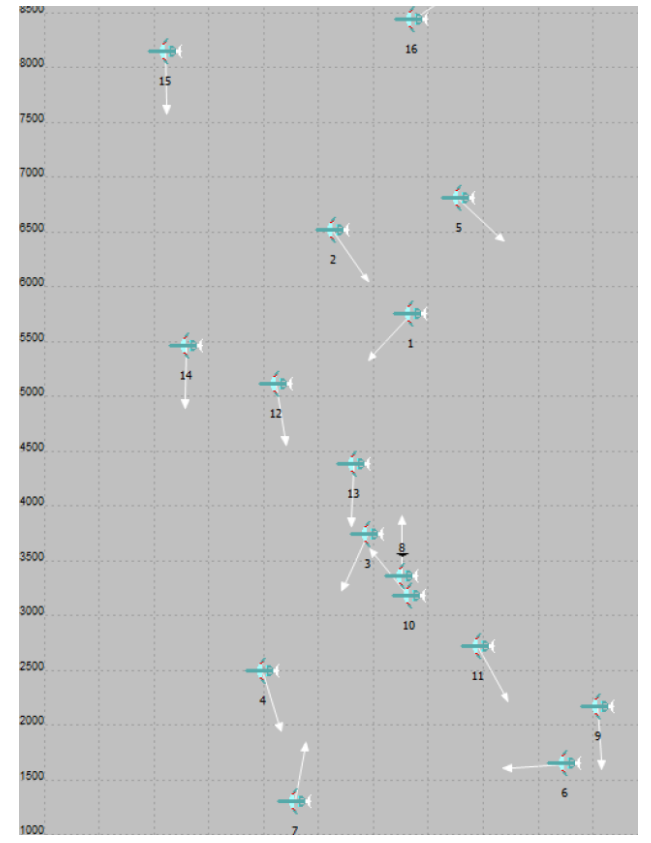

(b) Topology at $2 \mathrm{~min} 42 \mathrm{sec}$

Figure 6.2: Topology Change During Optimization 
have changed in the FANET topology, rendering the provided solution out-of-date and most likely no longer applicable. The controller will then prove to be simply useless most of the time if not all the time. The topology change during optimization is illustrated in Figure 6.2. We saw in the scenario studied in Section 5.3.1 that the optimization runtime for the static 8-flow 16-node network depicted in Figure 6.2(a) (Figure 5.1) is 2 min 42 sec. We now add mobility typical of a FANET, as described in more detail in [106]. Figures 6.2(a) and 6.2(b) show the topology at times 0 min $0 \mathrm{sec}$ and $2 \mathrm{~min} 42 \mathrm{sec}$ respectively. The transmission range is $1400 \mathrm{~m}$. The nodes move according to a Random Waypoint (RWP) mobility model, with speeds within the $[50-60] \mathrm{m} / \mathrm{s}$ range. The change in topology is self-evident: at time $2 \mathrm{~min} 42 \mathrm{sec}$, when the optimal solution is made available, many links have been broken and new ones have been formed, bearing little resemblance with the topology upon which the optimization is based (topology at 0 min 0 sec). Using the NetAnalyzer [107] tool, we find that, on average, 0.63 links break per second and 0.51 new links are formed. This means that over the 2 min $42 \mathrm{sec}$ time span, 102 links were broken, and 82 new links formed. With the refined (more realistic) optimization model in Section 5.4.2 we saw that it takes 7 min $08 \mathrm{sec}$ (significantly longer than just 2 min $42 \mathrm{sec}$ ) to compute the optimal solution for a 12 -node network with a $45^{\circ}$ beamwidth. Therefore, this idea of having a central controller is not a promising avenue to explore further in MANETs.

A way around having a central controller is to adopt a sort of centralizeddistributed approach whereby all nodes have a global view of the network and they are able to compute an optimal solution based on that global view. Even then, each node would still take a while to compute the optimal solution, and by the time it is available it will likely be obsolete and probably counter-productive. Furthermore, this approach will come at the cost of considerably high overhead in terms of control packets since it will require the dissemination/flooding of additional control messages necessary for the global view of the network topology. In fact, even the central controller approach will have this high overhead as well because the additional and necessary control messages will need to be forwarded to the controller. This high overhead issue is why we rejected the idea of a proactive routing protocol for MANETs in a previous work [8]. In effect, proactive protocols learn about the complete topology by means of additional topology control messages, as would any solution based on running an optimization algorithm. 
In a nutshell, as far as MANETs are concerned, a centralized approach, either with a central controller or distributed controllers, where an optimal solution has to be computed, is a poor approach for two main reasons: increased overhead and impracticality due to time-consuming computations. Now, instead of a centralized or a centralized-distributed approach, we can adopt a completely distributed and heuristic approach. This is discussed in the next section.

\subsection{A Distributed Heuristic Approach}

With a distributed and heuristic approach, we can build on an existing routing protocol of ours, the RGR [8] protocol, that has already proved to be best suited for Omni-MANETs and SBA-MANETs.

This approach is not to suggest that the optimization model becomes useless. Instead, we propose to use the model in more indirect way. In solving the model, we have learned some important lessons that we are now going to exploit to drastically change the way the paths are selected. The lessons learned are twofold yet tightly connected: avoid bridges and promote star nodes. With this promotion of star nodes, different flows are encouraged to "meet" along their respective paths. However, these "meetings" are tightly regulated: they are limited by the number of beams available at the meeting points (star nodes), and different flows must be serviced by different beams.

Simply put, a star node is a node that uses its MPR capability at one point of time to receive multiple packets from different flows before using its MPT capability at a subsequent point of time to forward those very packets. With a star node, many packets from distinct traffic flows concomitantly travel two hops in minimal time, which is obviously a desirable effect for reducing the E2E delay. A bridge is a beam that forwards or receives two or more packets, from different flows, that have arrived at a given node and have been ready to be forwarded or received at overlapping times, resulting in a queue (waiting delay) for that beam. 
The proposed protocol is going to build on RGR. RGR was introduced/described in Section 2.2.4. Similar to RGR, the route discovery is to be performed by means of RREQ, RREP, etc. Route repair is still ensured by a switch to GGF mode. However, in order to avoid bridges and promote stars in the selected routes, for the purpose of E2E delay reduction, the proposed algorithm is going to have a few novel points. One is that the nodes should now share, as soon as they know it, which flows they are involved in. In addition, the nodes should share which beams are being used or when they are scheduled to be used and for what flows. This information sharing must occur as frequently as possible/reasonable, inasmuch as it is very likely to change quickly due to the high mobility of the nodes. In the end, this information will help the source choose, among the discovered routes, which one avoids bridges the most, and which one forms stars the most. The new information (flows, beams, etc.) can be shared by means of HELLO, RREQ, and RREP messages. The format of our current HELLOs, RREQs, and RREPs will therefore have to change in order to piggyback and disseminate the new information. In the GGF mode, unlike RGR where the next hop is chosen based on the neighbor that is deemed to have a better progression toward the destination, the next hop selection is now going to be made in a way that takes into account bridge avoidance and star formation.

To illustrate, let us assume two traffic flows: flow 1 from $S 1$ to $D 1$, and flow 2 from $S 2$ to $D 2$. In the scenario depicted in Figure 6.3, the selected routes, as per our proposed algorithm, are:

flow 1 (represented by solid green arrows in the figure): $S 1 \rightarrow A \rightarrow B \rightarrow D 1$

flow 2 (represented by solid red arrows in the figure): $\quad S 2 \rightarrow A \rightarrow E \rightarrow D 2$

In this scenario, $S 1$ and $S 2$ both launched route discovery in reactive mode and ultimately discovered the following routes:

$S 1$ discovered one route: $S 1 \rightarrow A \rightarrow B \rightarrow D 1$

$S 2$ discovered three routes: $S 2 \rightarrow A \rightarrow B \rightarrow D 2, S 2 \rightarrow A \rightarrow C \rightarrow D 2$, and $S 2 \rightarrow A \rightarrow E \rightarrow D 2$

In the case of $S 1$, since only one route is discovered, that route is naturally the one selected. $S 2$, on the other hand, discovers three routes. The third route is the one that is selected because the first two result in a bridge being formed 


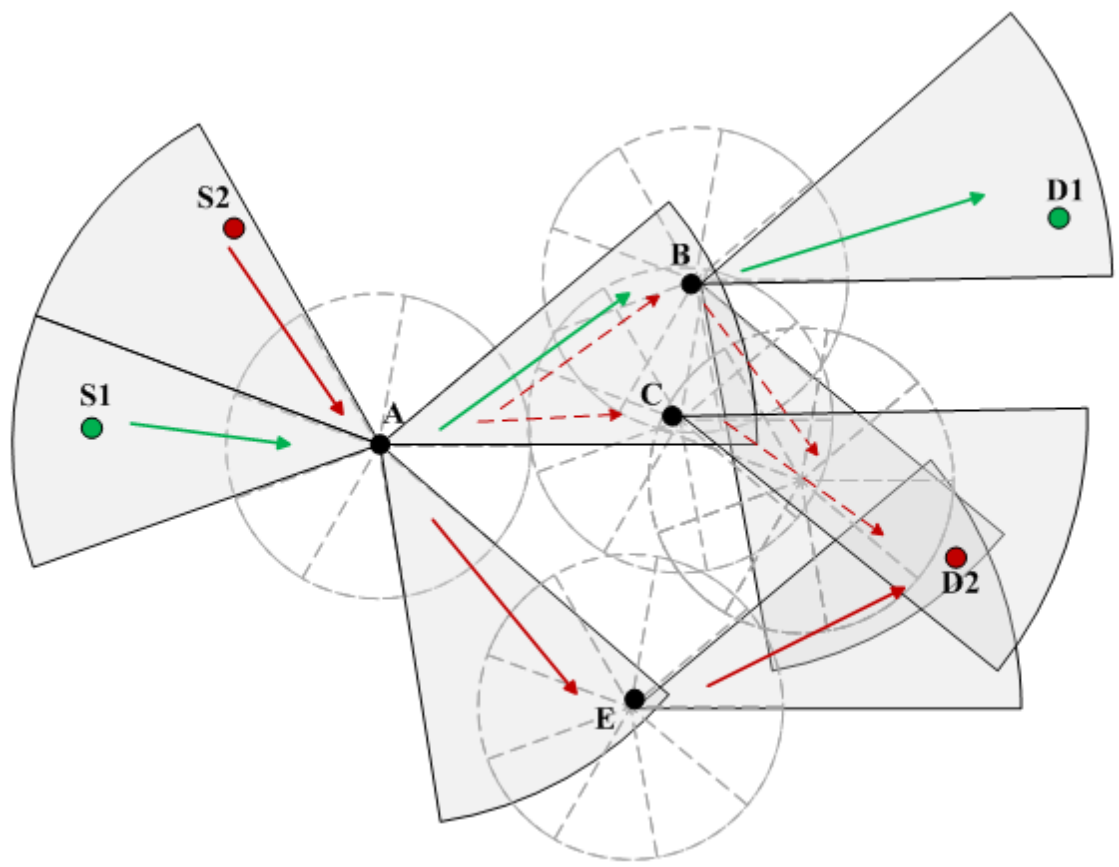

Figure 6.3: Route in Reactive Mode

on a beam of node $A$. In effect, nodes $B$ and $C$ are reachable by the same beam of node $A$. Since that beam is already used by flow 1 for the $A \rightarrow B$ link, using that same beam for flow 2 (for Link $A \rightarrow B$ or for Link $A \rightarrow C$ ) will result in a bridge, hence a waiting delay for either flow. In order to spare either flow that delay, $S 2$ chooses a route that uses a different beam of $A$ (the two routes that are not selected by $S 2$ are depicted with red dashed line in Figure 6.3). Node $A$ then becomes a star node. The desirable consequence is that both flows are now serviced in parallel (simultaneously), and therefore the waiting delay that would have occurred due to a bridge is now eliminated. These delay savings along the paths, when added up, should result in a considerable E2E delay reduction overall.

In the above-described scenario, it is assumed the path for flow 1 is discovered before the path for flow 2. That is how flow 2 is able to make an informed decision to avoid the beam that is already set to service flow 1 at node $A$. In the case where the path for flow 2 is discovered first, the chosen route could well be $S 2 \rightarrow A \rightarrow B \rightarrow D 2$. In this case, when the only path for flow 1 is discovered next, a decision will have to be made to re-route flow 2 in order to avoid the bridge. The re-routing policy/mechanism and all the parties that need to be involved in it are yet to be 
thoroughly defined.

Similar to RGR, our routing protocol will switch to GGF mode when a link of a pre-discovered route breaks while a packet is being forwarded. Unlike RGR, where the packet would be forwarded to the neighbor that is closest to (or moving faster toward) the destination, here such a neighbor will be avoided if it occasions a bridge to be formed. Figure 6.4 depicts such a scenario. Here it is assumed that the link $A \rightarrow B$ of flow 1 breaks, therefore node $A$ switches to GGF mode. Node $A$ has three neighbors: $C, E$, and $F . F$ is the neighbor that is closest to Destination $D 1$. In RGR, $A$ would forward the packet to $F$. But $F$ is reachable through a beam that is already servicing flow 2 through node $C$. Therefore, forwarding flow 1 's packet to $F$ would result in a bridge. The same is true if the packet is forwarded to node $C$ that is the second closest to destination $D 1$. In order to avoid the bridge, $A$ forwards flow 1's packet to neighbor $E$ instead. Node $A$ now forms a star. The packet will eventually reach the destination via a longer route that still has a better delay than a shorter route that has bridge-induced queuing delays.

The bottom line is that we are going to try to avoid bridge-induced queuing delays as much as possible both in reactive mode and in GGF mode. In the event that a bridge is unavoidable because there is no other viable option, then we will have to bear the resulting delay like in RGR. However, thanks to the MPR/MPT capability, we expect to have the option to avoid bridges a few times; giving us a reduced E2E delay overall. In effect, having the MPR/MPT capability gives us the privilege to discover more routes (hence options) to begin with. Two or more RREPs that arrive on an omnidirectional antenna, though in far-apart directions, would collide, and routes would not be discovered. With a single-beam directional antenna, deafness would impede the discovery of alternative routes to some extent.

What we propose is to design a protocol that encompasses the MBA-delay-saving features discussed above. We are going to derive some metrics that will measure the degree of less-bridge-more-star-ness (LBMS factor). And based on these metrics, the discovered routes will be ranked and thus selected. 


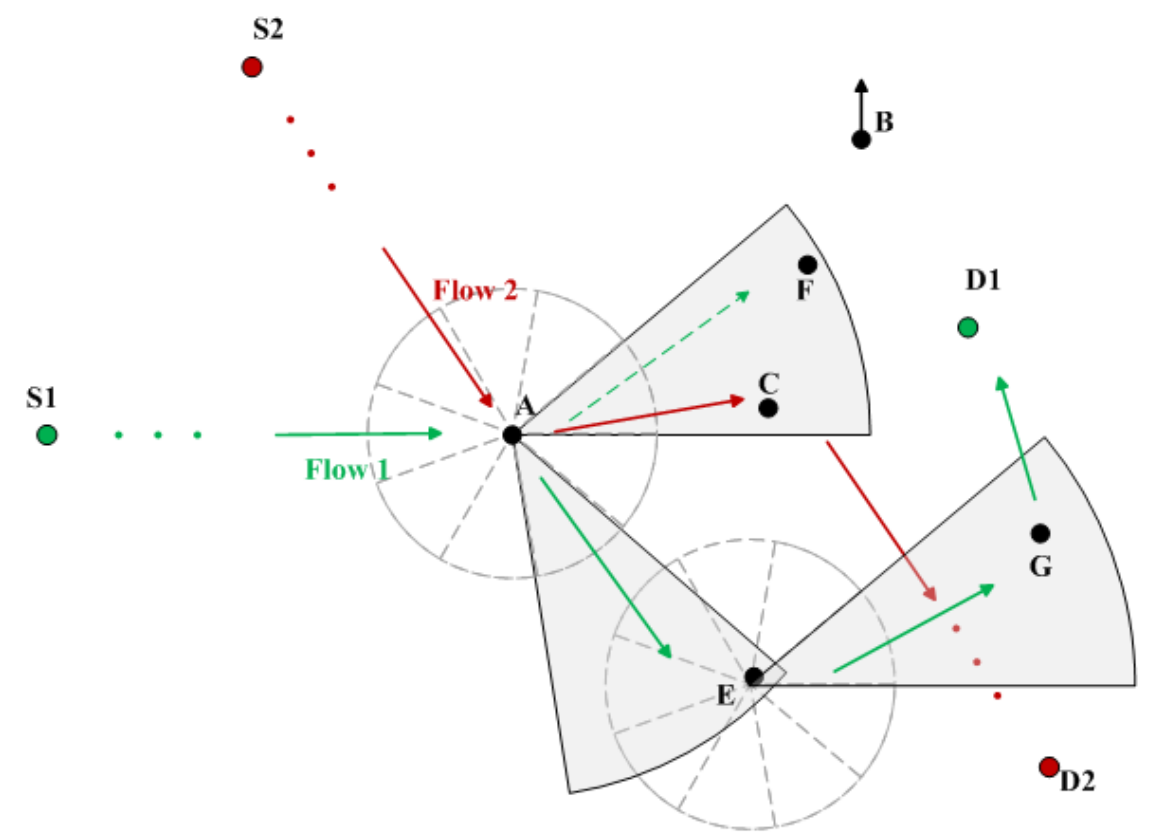

Figure 6.4: Route in Greedy Geographic Mode

\subsection{The Next Step}

In this chapter, we propose a Delay-Reducing Routing protocol that fully exploits Multi-Beam Antennas (MBA-DRR) in multi-flow ad hoc networks. The benefits of this protocol also apply to other types of MBA-based ad hoc networks, both mobile and static. As explained previously in Sections 6.2 and 6.3, we adopt a distributed heuristic approach based on RGR [8], the Reactive-Geographic hybrid Routing protocol that, in its turn, is based on the standard AODV routing protocol. MBA-DRR departs from RGR/AODV in that it: a) specifically targets the reduction of E2E delay, b) uses/exploits multi-beam antennas, and c) uses a routing metric that differs from the typical shortest-route. In effect, in MBA-DRR, we promote the formation of star nodes and avoid bridge nodes as much as possible. Therefore, we select routes that feature more star nodes and fewer bridge nodes, and whose bridge-induced delay, if any, is anticipated to be the shortest. Ultimately, our strategy should reduce the queuing delay for the delivered packets. To this effect, we define two metrics to rank the routes: the Star to Bridge Ratio (SBR) and the Practical Hop Count (PHC). 


\subsection{The Metrics}

The SBR metric is defined as the number of stars over the number of bridges (Equation 6.1). To define the PHC, we see the rank of a flow in a bridge as an additional hop that that flow goes through to account for the delay spent while waiting for its turn (in a queue) to occupy the beam/link. We assume the worst-case scenario whereby the flow of interest is ranked last for the occupation of the link. It follows that the PHC is defined as in Equation 6.2.

$$
\mathrm{SBR}=\frac{\text { Number of Star Nodes }}{\text { Number of Bridges }}
$$

PHC $=$ Traditional Hop Count + Number of Flows in Bridges - Number of Bridges

In the computation of the PHC, the Traditional Hop Count is the hop count that appears in the routing table. The Number of Flows in Bridges count is the total number of flows that are "competing" for access in the bridges of the considered route. It can be easily seen that for a bridgeless route (Number of Bridges $=0$ ), the PHC is equal to the Traditional Hop Count. Note that the Number of Bridges count is subtracted in Equation 6.2. In fact, only additional flows that make a regular/simple link become a bridge are considered as additional hops. For $N$ flows going through one bridge, the worst-case additional delay is $N-1$. So if we generalize this for the route, it becomes the subtraction of Number of Bridges from the Number of Flows in Bridges as seen in Equation 6.2.

In the computation of SBR, four cases arise:

- Case 1: Number of Bridges $=0 \&$ Number of Star Nodes $\neq 0 \Longrightarrow \mathrm{SBR}=$ infinite. The route is preferred to any other route. If compared with another SBR-infinite route, preference is given to the one with the smallest PHC.

- Case 2: Number of Bridges $=0 \&$ Number of Star Nodes $=0 \Longrightarrow$ Invalid SBR value. When compared with another route, except for an infinite-SBR route (Case 1), the route with the smallest PHC is preferred. 
- Case 3: Number of Bridges $\neq 0$ \& Number of Star Nodes $\neq 0 \Longrightarrow$ Valid and non-zero SBR value. Equation 6.1 is used. When compared with an infiniteSBR route (Case 1), the latter is preferred. When compared with another route with a valid non-zero value, the route with the highest SBR is preferred. Otherwise, the route with the smallest PHC is preferred.

- Case 4: Number of Bridges $\neq 0 \&$ Number of Star Nodes $=0 \Longrightarrow$ Valid and null SBR value. When compared with another route, except for an infinite-SBR route (Case 1), the route with the smallest PHC is preferred. This case proceeds similarly to Case 2 .

Table 6.1 presents the above four SBR cases and all the resulting route comparison cases that can be encountered. It shows which route is chosen. Digit 1 in the table means that the route with the infinite SBR (i.e. Case 1) is preferred.

Table 6.1: The SBR/PHC Measure

\begin{tabular}{|c|c|c|c|c|}
\hline & $\begin{array}{c}\text { Infinite SBR } \\
\text { (Case 1) }\end{array}$ & $\begin{array}{c}\text { Invalid SBR } \\
(\text { Case 2) }\end{array}$ & $\begin{array}{c}\text { Valid Non-Zero } \\
\text { SBR (Case 3) }\end{array}$ & $\begin{array}{c}\text { Valid Zero } \\
\text { SBR (Case 4) }\end{array}$ \\
\hline $\begin{array}{c}\text { Infinite SBR } \\
\text { (Case 1) }\end{array}$ & Smallest PHC & 1 & 1 & 1 \\
\hline $\begin{array}{c}\text { Invalid SBR } \\
\text { (Case 2) }\end{array}$ & 1 & Smallest PHC & Smallest PHC & Smallest PHC \\
\hline $\begin{array}{c}\text { Valid Non-Zero } \\
\text { SBR (Case 3) }\end{array}$ & 1 & Smallest PHC & Highest SBR & Smallest PHC \\
\hline $\begin{array}{c}\text { Valid Zero } \\
\text { SBR (Case 4) }\end{array}$ & 1 & Smallest PHC & Smallest PHC & Smallest PHC \\
\hline
\end{tabular}

Table 6.1 constitutes what we refer to as the $S B R / P H C$ measure. This measure will help to compare different routes with the goal of choosing the one that facilitates lower delay for all the flows. This measure gracefully degrades to shortest-route selection as the number of flows diminishes. In the limiting case of a single flow for instance, the SBR/PHC measure simply selects the shortest route. The concepts of stars and bridges only apply in the presence of multiple flows.

Nothing can be said about the transitivity of the SBR/PHC measure (if route $A$ is better than route $B$, and route $B$ is better than route $C$, does that imply that 
route $A$ is also better than route $C$ ?). The routes are compared two at a time as they are apprised of. In that sense, this is a greedy approach.

Note that, unlike RGR/AODV which checks traditional hop counts (and updates if the new hop count plus one is smaller than the existing one), MBA-DRR checks the SBR/PHC measure. This is a fundamental shift in selection criteria. This change is a direct consequence of a major observation from our preliminary work presented in Chapter 5. In effect, our preliminary work showed that, to reduce the average E2E delay in multi-flow environments (our ultimate objective's environment), longer routes are usually preferred when they feature more stars and fewer bridges. The SBR/PHC measure helps to quantify the notion of more stars and fewer bridges in relation with E2E delay.

Going forward, by star node and bridge, we actually mean potential star node and potential bridge. Given that transmissions (as well as receptions) are assumed to be asynchronous across the network, the stars/bridges are only expected to be potentially so, inasmuch as multiple flows involved in an anticipated (potential) star/bridge will not always result in an actual star/bridge. When they do, we will reap the benefits of star promotion and bridge avoidance in the average E2E delay of multi-flow scenarios. This reasoning is only considering the transmission of data packets.

\subsection{New Concepts and Changes from RGR}

MBA-DRR introduces a few new concepts such as: Beams (to next hop and to previous hop where applicable), Star Counter, Bridge Counter, Star to Bridge Ratio, and Practical Hop Count. In the routing tables, the new fields added are: Beam to Next Hop (B2NH), Beam to Previous Hop (B2PH), Star Counter (StarCnt), Bridge Counter (BridCnt), and Number of Flows in Bridges (BridNumFlows).

The addition of $\mathrm{B} 2 \mathrm{PH}$ will help identify stars and bridges. The beams used will tell a node whether it is a (potential) star/bridge node or not. A node will determine that it is a potential bridge if it uses the same beam for two or more different flows. A node will determine that it is a potential star if it uses two or more pairs of beams to service two or more flows. In practice, a node realizes that it 
is a star node if it has at least two $<\mathrm{b} 2 \mathrm{ph}, \mathrm{b} 2 \mathrm{nh}>$ distinct pairs. Similarly, a node realizes that it harbors a bridge if a certain beam appears twice or more in its table, no matter the direction (B2PH or B2NH). It follows that: First, a node can harbor multiple bridges. This reality is taken into account by considering the number of flows involved in bridges. In reality, this number corresponds to the number of links. Secondly, a node can be a star and harbor one or more bridges at the same time.

Only data packets update beams in the table. Control packets (RREQs, RREPs, etc.) do not update. The update is only made when actual data is crossing the node.

AODV and RGR have a provision on the propagation to RREQs that inherently limits the number of routes that can be discovered. In effect, as per AODV/RGR, an RREQ with the same ID and originator cannot be processed twice by a given node. But in general, and in the context of MBAs in particular, an RREQ with the same ID and originator might have taken a completely different route than the previously received one. Given that we want to learn of all possible routes, in MBA-DRR we allow a node to process an RREQ with the same ID and originator as a previously received one; as long as it has not gone through the current node before. Otherwise it creates a loop, which we want to avoid. The way to avoid these loops is by keeping track of all nodes that a given RREQ goes through. More details on the necessary bookkeeping to achieve this goal will follow in Section 6.8.3.

We do not expect MBA-DRR to result in the absolute optimal routes being selected because the route discovery is not perfect. There are a few practical/physical limitations that will impede the discovery of certain routes. But the philosophy of star/bridge, when followed, albeit on a best-effort basis, is expected to result in a significant improvement in latency. Amongst the practical/physical limitations are: rx-mode/tx-mode switching, medium contention, and collisions. For instance, if a node is switched to tx-mode, it could miss a given RREQ (which is a broadcast packet, hence not retransmitted unless a route is not found in the end) and that will be at least one route that will not be discovered. In the same vein, medium contention and collisions prevent some packets from being transmitted/received. Because of this, some RREQ/RREP will be lost and some routes will not be learned of. 
Note that there is no SBR or PHC field added to the routing table entries. Instead, as already mentioned, we have a star count, a bridge count, and a number of flows in bridges. Using these three numbers/counts, the SBR/PHC measure is computed when an update/selection decision is to be made. The fields of the routing table are shown in Table 6.2, with the new (MBA-DRR-specific) fields shaded.

Table 6.2: Routing Table Fields in MBA-DRR

\begin{tabular}{|l|c|c|c|c|c|c|c|c|}
\hline \multicolumn{8}{|c|}{ Routing Table } \\
\hline Dest & DestSeq\# & NextH & HopCnt & B2PH & B2NH & StarCnt & BridCnt & BridNumFlows \\
\hline
\end{tabular}

\subsection{Message Formats}

RREQ and RREP generally keep the same format as in RGR/AODV. However, we make them propagate the star and bridge counters and the number of flows in bridges. These three numbers/counters will be used by source nodes to decide on the routes to choose/update. In addition to these three new fields, the RREQ also maintains a list of already visited nodes. Therefore, we are adding four fields and three fields to the RREQ and RREP data structures respectively as shown in Tables 6.3 and 6.4 .

Table 6.3: RREQ Fields

\begin{tabular}{|l|c|c|c|c|c|c|c|c|c|}
\hline \multicolumn{10}{|c|}{ RREQ } \\
\hline RREQ_ID & Dest & DestSeq\# & Src & SrcSeq\# & HopCnt & StarCnt & BridCnt & BridNumFlows & ListVisitedNodes \\
\hline
\end{tabular}

Table 6.4: RREP Fields

\begin{tabular}{|l|c|c|c|c|c|c|}
\hline \multicolumn{5}{|c|}{ RREP } \\
\hline Dest & DestSeq\# & Src & HopCnt & StarCnt & BridCnt & BridNumFlows \\
\hline
\end{tabular}


StarCnt represents the number of star nodes thus far in the path. Initially, the source node (originator of the RREQ) or the destination node (originator of the RREP) examines its routing table and determines whether or not it is a star node. If it is, it sets StarCnt to 1; otherwise it sets it to 0. Likewise, every intermediate node receiving the RREQ or RREP will examine its routing table and determine whether it is a star node after performing any necessary routing table updates. The result of this examination will decide whether to increment StarCnt. The same idea applies to BridCnt, except now we are counting bridges instead of stars. Note that the same node may harbor multiple bridges since each of its beams can become a bridge. BridNumFlows represents the total number of flows involved in all the bridges encountered thus far. For instance, before an RREP is forwarded by an intermediate node, it might have gone through 2 bridges: one bridge having 2 flows, and the other having 3 flows. If the beam on which the RREP is going to be forwarded through is also a bridge of 4 flows, then BridNumFlows will be set to 9 in the RREP. This BridNumFlows count will serve in the computation of the PHC, as mentioned earlier. ListVisitedNodes represents the list of nodes (i.e. their IP addresses) that the RREQ has already visited. When a node receives an RREQ, it first checks whether its own address is on this list. If it is, then the RREQ is simply dropped without further processing. If the current node's address is not on the list, then it is added before the RREQ is forwarded when forwarding is appropriate.

When a source node receives an RREP, it learns about the number of stars and bridges on the reverse route and assumes that, by symmetry, the same star/bridge conditions apply to the forward route. In the event that conditions change while (or before) the forward route is being used, the source will not be aware of these changes. Eventually, a link will break (thanks to the high mobility of the nodes) and a new and more up-to-date route will be discovered.

\subsection{Protocol Operation}

A good extent of MBA-DRR's operation is very similar to RGR/AODV. The major change is that MBA functionality is now used and one key route selection criterion is changed. These changes justify the introduction of a few new variables and metrics. 


\subsubsection{Maintaining Sequence Numbers}

Every route table entry at every node includes the latest information available about the sequence number for the IP address of the destination node for which the route table entry is maintained. This sequence number is called the Destination Sequence Number. It is updated whenever a node receives new information about the sequence number from RREQ, RREP, or RERR messages that may be received related to that destination.

\subsubsection{Route Table Entries Update/Creation}

When a node receives a control packet from a neighbor, or creates or updates a route for a particular destination, it checks its route table for an entry for the destination. In the event that there is no corresponding entry for that destination, an entry is created. The sequence number is either determined from the information contained in the control packet, or else the valid sequence number field is set to false. The route is only updated if the new sequence number is either:

(i) higher than the destination sequence number in the route table, or

(ii) the sequence numbers are equal, but the SBR/PHC measure is in favor of the new route, or

(iii) the sequence number is unknown.

\subsubsection{RREQs}

A node initiates an RREQ when it determines that it needs a route to a destination and does not have one available. This can happen if the destination is previously unknown to the node, or if a previously valid route to the destination expired or is marked as invalid. The Destination Sequence Number field in the RREQ message is the last known destination sequence number for this destination and is copied from the Destination Sequence Number field in the routing table. If no sequence number is known, the unknown sequence number flag is set. The Originator Sequence Number in the RREQ message is the node's own sequence number, which is incremented prior to insertion in an RREQ. The RREQ ID field is incremented by one from the last RREQ ID used by the current node. Each node maintains only one RREQ ID. 
Note that the RREQ ID is a sequence number that uniquely identifies a particular RREQ when taken in conjunction with the originating node's IP address. This is different and serves a different purpose from the Originator Sequence Number that is the current sequence number to be used in the route entry pointing towards the originator of the RREQ. The Hop Count field is set to zero. As already mentioned, MBA-DRR adds some new information to an RREQ in the form of four new fields: Star Count, Bridge Count, Number of Flows, and List of Visited Nodes. The Star Count is initialized to 1 or 0 depending on whether the source node (originator of the RREQ) is a star node or not. The Bridge Count is initialized to the number of bridges that the source node forms. The Number of Flows field is initialized to the total number of flows in those bridges. The List of Visited Nodes is initialized with the IP address of the originator of the RREQ. To prevent unnecessary network-wide dissemination of RREQs, the originating node uses an expanding ring search technique.

When a node receives an RREQ, it checks if the node is a bridge with respect to the beam where the RREQ is received only; as that is the only beam of interest at this point. It then creates (or updates) a route to the previous hop with an invalid number for the sequence number, the star/bridge counts, and the number of flows.

In RGR, there is the notion of "safe distance" that dictates whether a node is to forward a received RREQ or not, based on the distance that separates the current node and the node from which the RREQ is received (previous hop). This notion was put in place in RGR to discover the most stable routes (i.e. routes that are not on the brink of breaking). We remove this "safe distance" feature in MBA-DRR because our main concern is to discover as many routes as possible and make the selection solely based on the SBR/PHC measure. Keeping the "safe distance" provision in place would possibly preclude the discovery of routes that could be delay-advantageous though short-lived.

In the same vein, in MBA-DRR we remove the "Already Received" condition on RREQ processing. This "Already Received" condition prevents a node from processing an RREQ that has the same originator and ID twice despite having traversed different paths. In fact, in RGR, the node that receives an RREQ would 
check to determine whether it has received an RREQ with the same Originator IP Address and RREQ ID within at least the last PATH_DISCOVERY_TIME. If such an RREQ has been received, the node discards the newly received RREQ. This is the "Already Received" condition. This condition precludes the discovery of many alternative and potentially better routes delay-wise. Recall that the optimal solution in Chapter 5 tests absolutely all possible routes. However, if we just remove this condition, we would run the risk of RREQ loops. To avoid such loops, we maintain a list of traversed nodes within the RREQ. Each node that receives an RREQ checks whether or not its address is already in that list. If it is, then the RREQ is simply dropped to avoid loops. If the address is not yet present in the list, the node can forward the RREQ after adding its address to the list.

In the case of an RREQ that does not have the current node's address already in its List of Visited Nodes, the current node proceeds as follows. It first increments the hop count value in the RREQ. It also updates the star/bridge counts and the number of flows if appropriate. Then, the node searches for a reverse route to the Originator IP Address. If need be, the route is created or updated using the Originator Sequence Number, the star/bridge counts, and the number of flows from the RREQ. This reverse route will be used if the node receives an RREP back to the node that originated the RREQ. Moreover, the current node can use the reverse route to forward data packets in the same way as for any other routes in the routing table. Notice that the star/bridge counts and the number of flows are updated as valid numbers only for the route entry pertaining to the RREQ's originator, and not for the route entry pertaining to the previous intermediate hop. This is so because the counts and numbers received from the RREQ are cumulative numbers since the originator. The star/bridge counts and the number of flows directly relating to the neighbor (previous hop) will be received with a HELLO message eventually.

If the current node does not generate an RREP, and if the incoming IP header has a TTL (the hop limit) larger than 1, the node updates and rebroadcasts the RREQ. To update the RREQ, the TTL field in the outgoing IP header is decremented, the Hop Count field in the RREQ message is incremented, and the current node's IP address is added to the List of Visited Nodes field. The Destination Sequence number for the requested destination is set to the maximum of the corresponding 
value received in the RREQ message, and the destination sequence value currently maintained by the node for the requested destination. Finally, the Star/Bridge Counts and the Number of Flows are incremented depending on whether the current node realizes that it is a star/bridge node. This information is updated as soon as the node receives a control packet (RREQ or RREP) that it needs to forward. However, by the time the destination of the control packet receives it, the star/bridge condition of a particular intermediate node may have changed. The destination of the control packet will not apprise of this change right away. In this case, the destination of the control packet will make decisions based on outdated information; up until a link breaks (due to mobility or missed HELLOs) and a new route discovery is triggered.

Note that the forwarding node does not modify its maintained value for the destination sequence number, even if the value received in the incoming RREQ is larger than the value currently maintained by the forwarding node. This is a behaviour inherited from standard AODV. The authors do not explain why this is the case. We believe this behavior is justified as follows: a sequence number from an RREQ cannot be "trusted" since the very existence of said RREQ indicates that there is no valid route associated with that sequence number.

If the current node is in a position to generate an RREP, then it discards the RREQ. If intermediate nodes reply to every transmission of RREQs for a particular destination, it might turn out that the destination does not learn of a route to the originating node from the RREQ messages. A Gratuitous RREP flag is preemptively set in the RREQ by the originator to avoid this situation. Figure 6.5 outlines the main steps in the processing of an RREQ.

\subsubsection{RREPs}

A node generates an RREP if either it is itself the destination, or it has an active route to the destination. In the latter case, an RREP is generated if the destination sequence number in the node's existing route table entry for the destination is valid and greater than or equal to the Destination Sequence Number of the RREQ, and the "destination only" flag is not set. If the existing route entry for the destination 


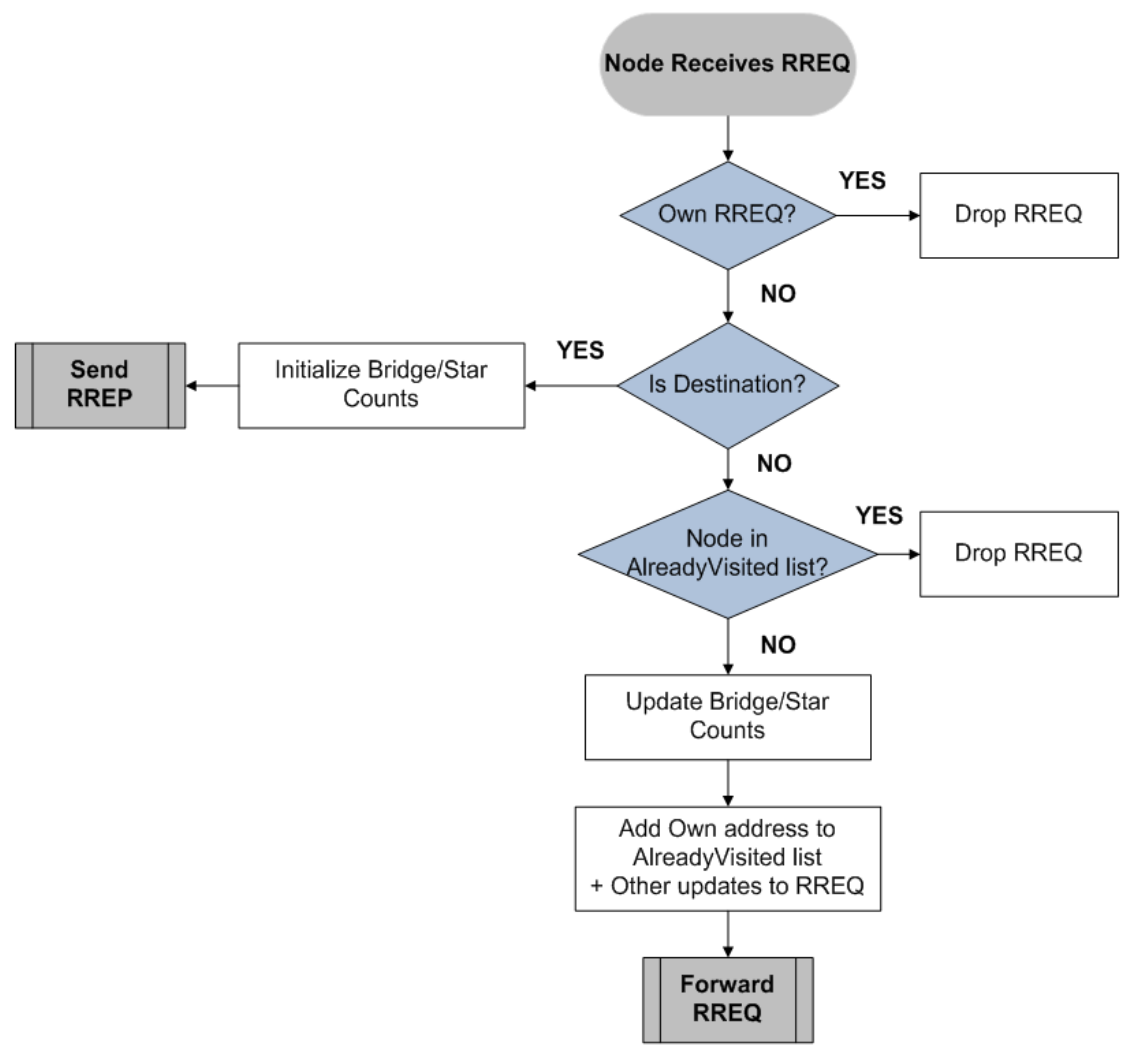

Figure 6.5: Flowchart for Processing RREQ

does not have a sequence number, no RREP is generated and the RREQ is forwarded instead. When generating an RREP message, a node copies the Destination IP Address and the Originator Sequence Number from the RREQ message into the corresponding fields in the RREP message. Similar to the case with RREQ, MBA-DRR does add some new information to the RREP as well, in the form of three new fields: Star Count, Bridge Count, and Number of Flows. The Star Count is initialized to 0 . The Bridge Count is initialized to the number of bridges that the destination node forms. The Number of Flows field is initialized to the total number of flows in those bridges. In the case of an intermediate node generating an RREP, the star/bridge counts and the number of flows are initialized to the values currently in the table entry for the corresponding destination node.

When a node receives an RREP message, it searches for a route to the previous hop. If needed, a route is created for the previous hop, with an invalid number for the sequence number, the star/bridge counts, and the number of flows. Next, the 
node increments the hop count value in the RREP. Moreover, the node increments the star/bridge counts and the number of flows if it determines, based on its current routing table, that it is a star node and/or harbors bridges.

Furthermore, the forward route for this destination is created if it does not already exist. If it already exists, the node compares the Destination Sequence Number in the message with its own stored Destination Sequence Number for the Destination IP Address in the RREP message. Upon comparison, the existing entry is updated only in the following circumstances:

(i) the sequence number in the routing table is marked as invalid in the route table entry, or

(ii) the Destination Sequence Number in the RREP is greater than the node's copy of the Destination Sequence Number and the known value is valid, or

(iii) the sequence numbers are the same, but the route is marked as inactive, or

(iv) the sequence numbers are the same, but the SBR/PHC measure is in favor of the new route.

Figure 6.6 outlines the main steps in the processing of an RREP.

\subsubsection{HELLOs}

Each node periodically broadcasts HELLO messages. A node should only broadcast HELLO messages if it is part of an active route. Every HELLO_INTERVAL milliseconds, the node checks whether it has sent a broadcast within the last HELLO_INTERVAL. If it has not, and it is part of an active route, it broadcasts an RREP with TTL $=1$, which is a HELLO message, with the RREP message fields set as in Table 6.5.

A node determines connectivity by listening for packets from its set of neighbors. If, within the past DELETE_PERIOD, it has received a HELLO message from a neighbor, and then does not receive any packets (HELLO messages or otherwise) from that neighbor for more than ALLOWED_HELLO_LOSS * HELLO_INTERVAL milliseconds, the node assumes that the link to this neighbor is currently lost, and 


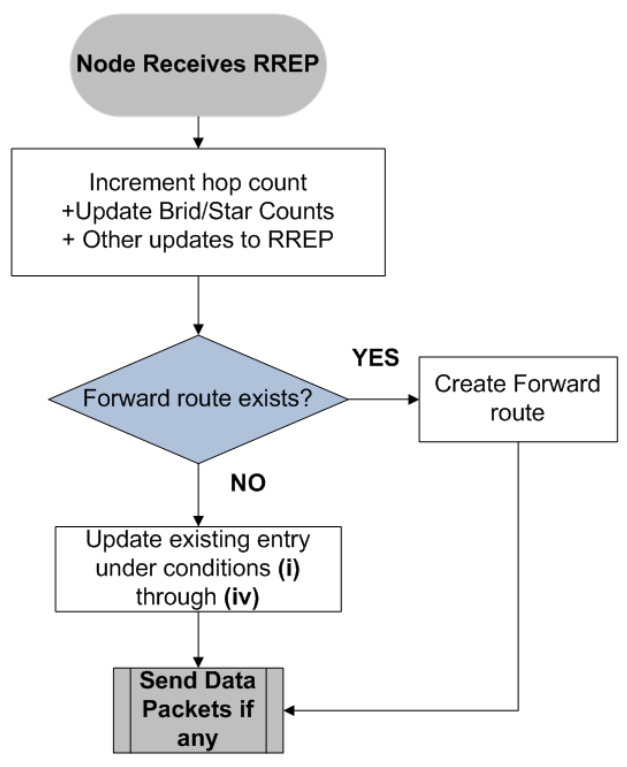

Figure 6.6: Flowchart for Processing RREP

Table 6.5: HELLO Message Fields Values

\begin{tabular}{|c|c|}
\hline Field & Value \\
\hline Destination IP Address & Broadcast IP address \\
\hline Destination Sequence Number & The node's latest sequence number \\
\hline Hop Count & 0 \\
\hline Lifetime & ALLOWED_HELLO_LOSS*HELLO_INTERVAL \\
\hline
\end{tabular}

takes appropriate action.

Whenever a node receives a HELLO message from a neighbor, the node makes sure that it has an active route to the neighbor, and creates one if necessary. If a route already exists, then the Lifetime for the route should be increased, and the star/bridge Counts and number of flows updated depending on the beam the HELLO is received on.

As already mentioned, a HELLO message is a special type of RREP with TTL set to 1 and destination IP address set to broadcast. We set all the new fields (Star Count, Bridge Count, and number of flows involved in bridges) to zero. Note that, 
like in RGR, the HELLO message will also include information about the direction of movement of the node and its current location (so do RREQs and RREPs).

\subsubsection{Route Break, GGF Mode}

Similar to RGR, MBA-DRR will switch to GGF mode when a link of a previously discovered route breaks while a packet is being forwarded. Note that the intermediate node where the link break occurs still sends out a Route Error (RERR) message to the source node (a behaviour inherited from AODV and RGR, see Sections 2.2.1 and 2.2.4) while switching to GGF. Unlike RGR, where the packet would be forwarded to the neighbor that is closest to (or moving faster toward) the destination, here such a neighbor will be avoided, assuming we can afford it, if it occasions a bridge to be formed. Because we want to avoid bridges (hence queues) as much as we can, preference will be given to a neighbor whose selection occasions the smallest bridge (in terms of the flows present in it) on the beam to that neighbor, provided that that neighbor shows some closeness or some movement toward the destination even if it is not the best in that regard. In a future work beyond this thesis, we shall explore other preference criteria. The neighbor to be chosen is a neighbor that results in the smallest bridge (in terms of the number of flows serviced) at the sender's (current node) end. The sender can detect such a bridge by simply realizing that the beam it uses to reach the receiver already harbors one or more different flows. Note that, from the sender's perspective, there is no way of knowing that this transmission will not result in a bridge at the next-hop receiver's end. In case of a tie between neighbors, the BMN (Best Moving Node), as defined in RGR, is chosen.

\subsection{Protocol Operation Example}

In this example, we consider the static 12-node network shown in Figure 6.7. Note that this is the same topology as presented in Figure 5.3 (Chapter 5); which was obtained by significantly constraining/altering ${ }^{1}$ the initial 16-node topology presented in Figure 5.1. The network has 6 flows as follows:

Flow 1: $1 \rightarrow 15$

Flow 2: $3 \rightarrow 13$

\footnotetext{
1 by removing nodes $5,9,7$, and 11
} 
Flow 3: $2 \rightarrow 14$

Flow 4: $16 \rightarrow 1$

Flow 5: $13 \rightarrow 4$

Flow 6: $14 \rightarrow 3$

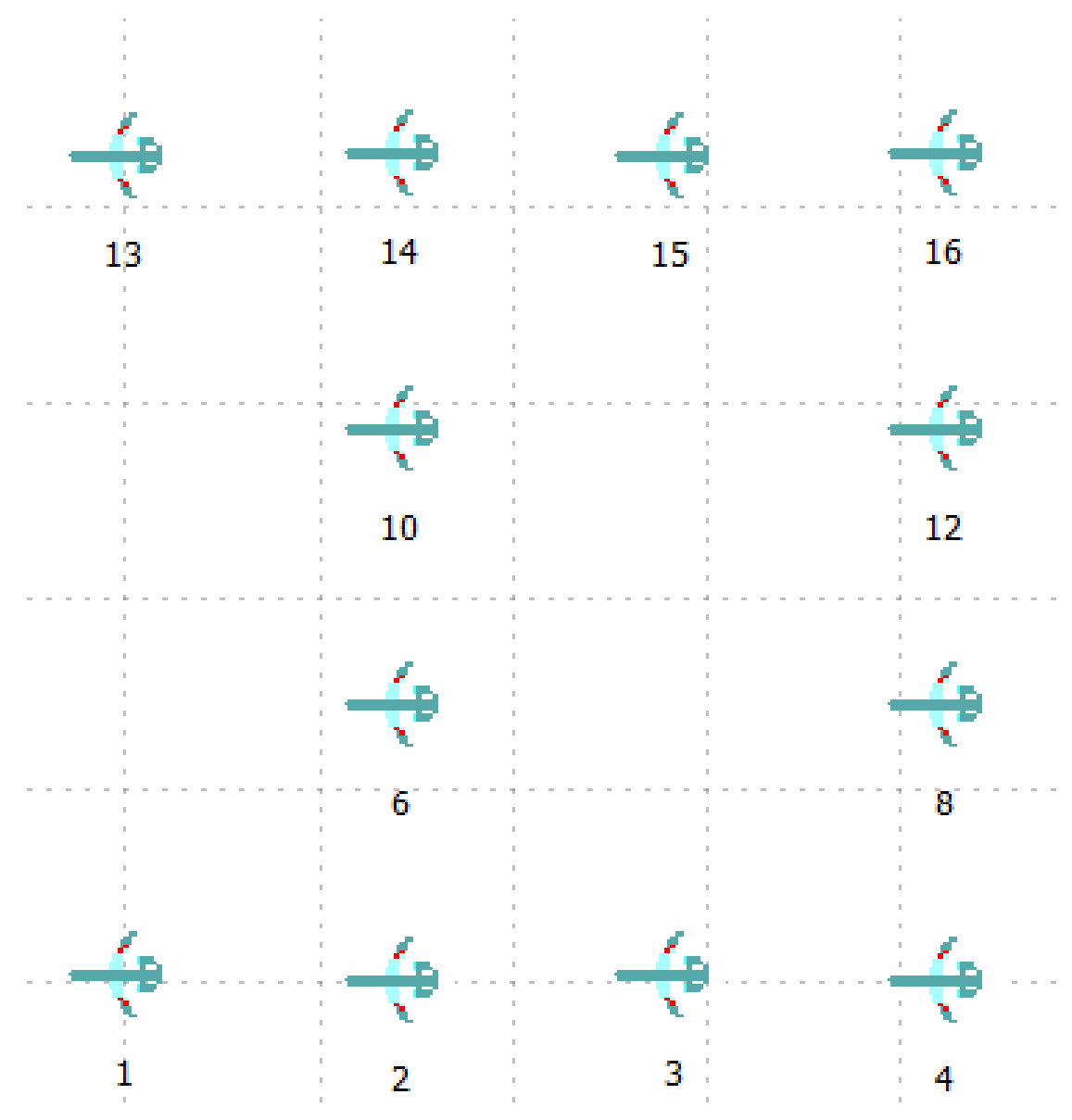

Figure 6.7: Static Scenario

All the nodes are equipped with 6-beam antennas with the configuration shown in Figure 6.8. We position the nodes in such a way that each node has at most one neighbor per beam. The nodes are positioned in such a way that node 2 for instance falls within sector 6 of node 1 , and node 1 falls within sector 3 of node 2 . All sectors/beams of interest will be presented in tables after data packets have started flowing. 


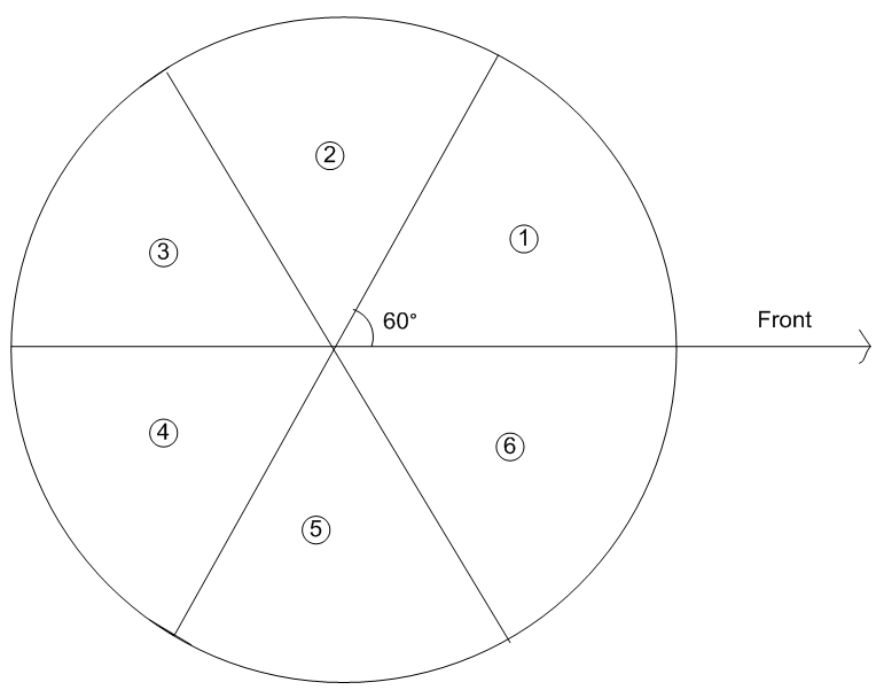

Figure 6.8: 6-Beam Configuration of the Nodes

Initially, no source node $(1,3,2,16,13$, and 14) has a route to its destination $(15,13,14,1,4$, and 3 respectively). Therefore, all the sources initiate a route discovery. Initially, the routing tables are all empty (See Table 6.6). The new/added fields (MBA-DRR specific) to the routing table are shaded.

Table 6.6: Initial State of the Routing Tables

\begin{tabular}{|c|c|c|c|c|c|c|c|c|}
\hline \multicolumn{10}{|c|}{ Initial Routing Table of all Nodes } \\
\hline Dest & DestSeq\# & NextH & HopCnt & B2PH & B2NH & StarCnt & BridCnt & BridNumFlows \\
\hline- & - & - & - & - & - & - & - & - \\
\hline
\end{tabular}

In the following, we present a few interesting points in the execution of: route discovery for all 6 flows, packet routing for all 6 flows, route break for flow 1, GGF for flow 1, and new route discovery for flow 1 . These points are representative of the bulk of the working of MBA-DRR. All the values and events presented here are obtained by tracking the packets in the OPNET simulator.

(a) The RREQ broadcast by node 1 is received at node 6 at time 0.0068 sec, while it is received at node 2 at 0.0073 sec. This difference is significant and it highlights an interesting feature of the MBA-DbMAC protocol proposed in 
Chapter 4: the decoupled broadcasting ${ }^{2}$. As per this feature, when a node has a packet to broadcast, it makes $N-1$ copies of that packet ( $N$ being the number of antenna sectors). It then sends each copy on each sector independently (in a decoupled fashion) as the medium becomes idle on the concerned sectors. What happened here is that node 2 was the first to broadcast its own RREQ. Node 1 heard it on its beam 1 and the node went into rx-mode before it got a chance to transmit its RREQ. Beam 2 of node 1 stayed idle the whole time. Therefore, when node 1 got a chance (after the rx-mode had ended) to broadcast its own RREQ, it only sent the copies of all other beams (including beam 2) except for beam 1 where it had to wait for an IFS (inter-frame space) period. This explains why the RREQ broadcast by node 1 is received at node 6 before it is received at node 2 . Both nodes 2 and 6 update their routing tables upon reception of this RREQ from node 1 . The routing table for node 6 is now as shown in Table 6.7. Note that node 6 had already received an RREQ from node 2 and therefore updated its table accordingly before it received the RREQ from node 1.

Table 6.7: Routing Table of Node 6, Step 1

\begin{tabular}{|c|c|c|c|c|c|c|c|c|}
\hline \multicolumn{10}{|c|}{ Routing Table of Node 6 } \\
\hline Dest & DestSeq\# & NextH & HopCnt & B2PH & B2NH & StarCnt & BridCnt & BridNumFlows \\
\hline 2 & 1 & 2 & 1 & - & - & - & - & - \\
\hline 1 & 1 & 1 & 1 & - & - & - & - & - \\
\hline
\end{tabular}

(b) Nodes 3, 1, and 6 receive the RREQ that node 2 has re-broadcast. Node 1 discards it, as a node cannot receive its own RREQ. Node 6 also discards the RREQ as it determines that it already has a route to node 1 (the originator) that is better than the new one. In effect, node 6 already has a route $6 \rightarrow 1$ to node 1 . The new RREQ means a new route $6 \rightarrow 2 \rightarrow 1$ to node 1 . Since both routes have the bridge count and the star count set to 0 , the SBR is invalid for both, and the selection comes down to the PHC which at this point is simply the hop count since there are no bridges in any of the routes. This is Case 1 presented in Section 6.5, or more precisely, it corresponds to cell (Case 1, Case

\footnotetext{
${ }^{2}$ This feature is better illustrated here with this practical example
} 
1) in Table 6.1.

The selection process keeps defaulting back to PHC then shortest-route for this entire first route discovery process for all the flows. This state of affair will change only once we have had data packets flowing and beams being updated.

Node 3, before re-broadcasting the RREQ, increments the hop count from node 1 and adds its own address to the list of already visited nodes (by this RREQ). The RREQ that leaves node 3 is shown in Table 6.8. This rebroadcast RREQ reaches node 2 again. Node 2 discards it, as it finds its own address on the already-visited-nodes list. However, an RREQ that comes from the same originator node 1 but went through node 6 before node 3 is accepted and rebroadcast. Remember that we allowed this behavior in order to explore as many potential routes as possible.

Table 6.8: RREQ Re-broadcast from Node 3

\begin{tabular}{|c|c|c|c|c|c|c|c|c|c|}
\hline \multicolumn{10}{|c|}{ RREQ re-broadcast from Node 3 } \\
\hline RREQ_ID & Dest & DestSeq\# & Src & SrcSeq\# & HopCnt & StarCnt & BridCnt & BridNumFlows & ListVisitedNodes \\
\hline 1 & 15 & - & 1 & 1 & 2 & 0 & 0 & 0 & $1,2,3$ \\
\hline
\end{tabular}

(c) Once the initial route discovery process is over, the selected routes are as follows:

Flow 1: $1 \rightarrow 2 \rightarrow 3 \rightarrow 8 \rightarrow 12 \rightarrow 15$

Flow $2: 3 \rightarrow 6 \rightarrow 10 \rightarrow 13$

Flow $3: 2 \rightarrow 6 \rightarrow 10 \rightarrow 14$

Flow $4: 16 \rightarrow 12 \rightarrow 8 \rightarrow 3 \rightarrow 2 \rightarrow 1$

Flow $5: 13 \rightarrow 14 \rightarrow 15 \rightarrow 16 \rightarrow 12 \rightarrow 8 \rightarrow 4$

Flow $6: 14 \rightarrow 10 \rightarrow 6 \rightarrow 3$

Note that half the routes are also the shortest routes. This is because there are no stars/bridges learnt of at this stage, therefore the SBR in MBA-DRR is invalid and the PHC falls back to the hop count (See Table 6.1 and Equation 6.2). 
Flows 4 and 5 did not choose their shortest route because it was never discovered due to physical limitations such as RREQs/RREPs being lost due to collision, mode mismatch, etc. The routing tables for all 12 nodes right before data starts flowing are presented in Table A.1 through Table A.12 in Appendix A.

(d) Beam numbers are updated in the tables as data packets begin to flow. Once all flows have completed the delivery of their first packet, the updated (beam numbers) routing tables of nodes 1,3 , and 6 are presented in Table 6.9 through Table 6.11 here. Refer to Table A.13 through Table A.21 in Appendix A for all the other nodes. Valid beam numbers indicate the passage of data flows. When a flow starts or ends at a given node, only the $\mathrm{B} 2 \mathrm{NH}$ is valid, and the corresponding $\mathrm{B} 2 \mathrm{PH}$ remains invalid, as there is no traversal of the node by that particular flow. For example, from node 3's routing table (Table 6.9), we can see that a flow whose destination is node 15 (flow 1) traverses the node from beam 4 (pointing to previous hop node 2 , as per the selected route shown in (c) above) to beam 1 (pointing to next hop node 8). At the same time, the flow whose source is node 14 (flow 6) ends at beam 3, as node 3 points to node 6 (previous hop on the route) using its beam 3 . The packet that was issued by node 14 arrived at node 3 via that beam 3 . Likewise, the flow whose destination is node 13 (flow 2) starts at beam 3 (pointing to next hop node 6 of that particular flow).

Table 6.9: Routing Table of Node 3

\begin{tabular}{|c|c|c|c|c|c|c|c|c|}
\hline \multicolumn{10}{|c|}{ Routing Table of Node 3 } \\
\hline Dest & DestSeq\# & NextH & HopCnt & B2PH & B2NH & StarCnt & BridCnt & BridNumFlows \\
\hline 8 & -1 & 8 & 1 & - & - & 0 & 0 & 0 \\
\hline 4 & -1 & 4 & 1 & - & - & 0 & 0 & 0 \\
\hline 14 & 1 & 6 & 3 & - & 3 & 0 & 0 & 0 \\
\hline 2 & 1 & 2 & 1 & - & - & 0 & 0 & 0 \\
\hline 16 & 1 & 6 & 5 & - & - & 0 & 0 & 0 \\
\hline 13 & 2 & 6 & 3 & - & 3 & 0 & 0 & 0 \\
\hline 6 & -1 & 6 & 1 & - & - & 0 & 0 & 0 \\
\hline 15 & 0 & 8 & 3 & 4 & 1 & 0 & 0 & 0 \\
\hline 1 & 1 & 2 & 2 & 1 & 4 & 0 & 0 & 0 \\
\hline
\end{tabular}

As we can infer from the tables (both here and in Appendix A), more than half the nodes are stars and harbor bridges of different sizes. Recall that for a node 
to be considered a star, it has to service at least 2 distinct flows on 2 distinct pairs of sectors. And, for a node to have a bridge, a given beam has to appear more than once in its table. The number of appearances is equal to the number of flows involved in the bridge. Node 3 (Table 6.9), for example, is a star. It services flow 1 and flow 2 in two distinct pairs of sectors: pair $<4,1>$ and pair $<1,4>$. However, given that the distinct pairs do have beams in common, node 3 also harbors bridges: a bridge on beam 4 and a bridge on beam 1, both consisting of two flows each. In addition, node 3 has a third bridge on beam 3 (as it appears twice in the table). Node 1 (Table 6.10), on the other hand, is not a star but does harbor a bridge on its beam 6 that services two flows (outbound flow 1 and inbound flow 4). Node 6 (Table 6.11) is a star and also harbors two bridges: on its beam 2 that services three flows (crossing flows 2, 3, and 6), and on its beam 6 that services two flows (crossing flows 2 and 6 ).

Table 6.10: Routing Table of Node 1

\begin{tabular}{|c|c|c|c|c|c|c|c|c|}
\hline \multicolumn{10}{|c|}{ Routing Table of Node 1 } \\
\hline Dest & DestSeq\# & NextH & HopCnt & B2PH & B2NH & StarCnt & BridCnt & BridNumFlows \\
\hline 14 & 1 & 6 & 3 & - & - & 0 & 0 & 0 \\
\hline 2 & 1 & 2 & 1 & - & - & 0 & 0 & 0 \\
\hline 16 & 1 & 2 & 6 & - & 6 & 0 & 0 & 0 \\
\hline 3 & 1 & 2 & 2 & - & - & 0 & 0 & 0 \\
\hline 6 & -1 & 6 & 1 & - & - & 0 & 0 & 0 \\
\hline 15 & 0 & 2 & 5 & - & 6 & 0 & 0 & 0 \\
\hline
\end{tabular}

Table 6.11: Routing Table of Node 6

\begin{tabular}{|c|c|c|c|c|c|c|c|c|}
\hline \multicolumn{10}{|c|}{ Routing Table of Node 6} \\
\hline Dest & DestSeq\# & NextH & HopCnt & B2PH & B2NH & StarCnt & BridCnt & BridNumFlows \\
\hline 14 & 1 & 10 & 2 & 5 & 2 & 0 & 0 & 0 \\
\hline 2 & 1 & 2 & 1 & - & - & 0 & 0 & 0 \\
\hline 16 & 1 & 10 & 4 & - & - & 0 & 0 & 0 \\
\hline 3 & 1 & 3 & 1 & 2 & 6 & 0 & 0 & 0 \\
\hline 13 & 2 & 10 & 2 & 6 & 2 & 0 & 0 & 0 \\
\hline 10 & -1 & 10 & 1 & - & - & 0 & 0 & 0 \\
\hline 15 & 0 & 10 & 4 & - & - & 0 & 0 & 0 \\
\hline 1 & 1 & 1 & 1 & - & - & 0 & 0 & 0 \\
\hline
\end{tabular}

(e) At some point, link $3 \rightarrow 6$ is declared broken by node 3 because it has not received any packet (HELLO or a flow-6 data packet) from node 6 within a 
given preset time period. This is the only way that a node realizes that a neighbor is unreachable at the Network-layer level. Given that the link between node 3 and node 6 is used in both directions, it is possible that the direction $6 \rightarrow 3$ has repeatedly gotten the short end of the stick (with either successive backoff periods for example, and/or finding node 3 turned in tx-mode as it is involved with other flows) when a packet has tried to travel in that direction. As a consequence, there has been no inbound stream at node 3 from node 6 for a period of time, leading to the invalidation of link $3 \rightarrow 6$. Moreover, if for a period of time, data packets from node 6 fail to reach node 3, the HELLOs are very likely to fail as well, especially since they are "one-shot" messages (as all broadcast messages) with no acknowledgment/retry at the MAC level.

Due to the above link invalidation (entry for route $3 \rightarrow 13$ still present in Table 6.9, the routing table of node 3 but marked invalid), a data packet from flow $2(3 \rightarrow 13)$ can no longer be forwarded to node 6 . A switch to GGF is performed. Node 3 considers all of its neighbors (based on the routing table presented above), excluding the one already declared unreachable (node 6). The only neighbors left for consideration are therefore nodes 2,4 , and 8 . Node 3 reaches node 2 by beam 4 which already services 2 flows (it is appearing twice in the table). Forwarding the packet to node 2 would therefore occasion a bridge of size 3 (the existing 2 flows + the new one). Node 3 reaches node 8 via beam 1 . And as can be seen from the routing table, beam 1 also is already servicing 2 flows, therefore, forwarding the packet to node 8 would also occasion a bridge of size 3 (the existing 2 flows + the new one). Finally, node 3 reaches node 4 via beam 6 . Beam 6 is not present in the table, therefore there is no bridge. As a result, the data packet is forwarded to node 4 as per the selection criterion of MBA-DRR. The existing but invalidated route to node 13 (via node 6 ) is expunged from the table after a certain time (DELETE_PERIOD). Any new data packet meant for node 13 will then trigger a new route discovery from node 3 .

(f) Due to the broken link seen above, node 3 initiates a new route discovery. At the end, it first discovers the exact same route that was invalidated before. This is normal because the nodes have not moved; it just happens that the 
packet coming from node 6 (an RREP on its final hop to source node 3) was received this time (unlike a previously missed HELLO). However, given that data traffic is already flowing in the network (hence there are active beams in routing tables of all nodes in the network), the received route actually has some meaningful metrics (star/bridge counts) to compute the SBR/PHC measure. But because it is the first route learnt, it is accepted without evaluating that SBR/PHC measure. Node 3 starts sending the packets using that route. The routing table of node 3 is now as shown in Table 6.12.

Table 6.12: Routing Table of Node 3 After Re-discovery, 1st Route

\begin{tabular}{|c|c|c|c|c|c|c|c|c|}
\hline \multicolumn{10}{|c|}{ Routing Table of Node 3 } \\
\hline Dest & DestSeq\# & NextH & HopCnt & B2PH & B2NH & StarCnt & BridCnt & BridNumFlows \\
\hline 8 & -1 & 8 & 1 & - & - & 0 & 0 & 0 \\
\hline 4 & -1 & 4 & 1 & - & - & 0 & 0 & 0 \\
\hline 14 & 1 & 6 & 3 & - & 3 & 0 & 0 & 0 \\
\hline 2 & 1 & 2 & 1 & - & - & 0 & 0 & 0 \\
\hline 16 & 1 & 6 & 5 & - & - & 0 & 0 & 0 \\
\hline 13 & 2 & 6 & 3 & - & 3 & 2 & 6 & 12 \\
\hline 6 & -1 & 6 & 1 & - & - & 0 & 0 & 0 \\
\hline 15 & 0 & 8 & 3 & 4 & 1 & 0 & 0 & 0 \\
\hline 1 & 1 & 2 & 2 & 1 & 4 & 0 & 0 & 0 \\
\hline
\end{tabular}

Subsequently, another RREP is received at node 3, signalling the discovery of another route from the same discovery. The received RREP is shown in Table 6.13.

Table 6.13: RREP Received by Node 3

\begin{tabular}{|c|c|c|c|c|c|c|}
\hline \multicolumn{7}{|c|}{ RREP Received by Node 3 } \\
\hline Dest & DestSeq\# & Src & HopCnt & StarCnt & BridCnt & BridNumFlows \\
\hline 13 & 1 & 3 & 5 & 4 & 10 & 26 \\
\hline
\end{tabular}

Now that there are two competing routes, the already learnt one and the new one, node 3 applies the SBR/PHC measure as follows: 
Since both routes have valid non-zero SBR (star count and bridge count non-zero for both), we will choose the route with the highest SBR (see Table 6.1).

$$
\begin{aligned}
& \text { Existing route: } \mathrm{SBR}=\frac{\text { Number of Star Nodes }}{\text { Number of Bridges }}=\frac{2}{6}=0.3 \overline{3} \\
& \text { New route: } \mathrm{SBR}=\frac{\text { Number of Star Nodes }}{\text { Number of Bridges }}=\frac{4}{10}=0.4
\end{aligned}
$$

Consequently, the new route is preferred to the old one, and the routing table is changed as shown in Table 6.14.

Table 6.14: Routing Table of Node 3 After Re-discovery, 2nd Route

\begin{tabular}{|c|c|c|c|c|c|c|c|c|}
\hline \multicolumn{7}{|c|}{ Routing Table of Node 3 } \\
\hline Dest & DestSeq\# & NextH & HopCnt & B2PH & B2NH & StarCnt & BridCnt & BridNumFlows \\
\hline 8 & -1 & 8 & 1 & - & - & 0 & 0 & 0 \\
\hline 4 & -1 & 4 & 1 & - & - & 0 & 0 & 0 \\
\hline 14 & 1 & 6 & 3 & - & 3 & 0 & 0 & 0 \\
\hline 2 & 1 & 2 & 1 & - & - & 0 & 0 & 0 \\
\hline 16 & 1 & 6 & 5 & - & - & 0 & 0 & 0 \\
\hline 13 & 2 & 8 & 5 & - & 1 & 4 & 10 & 26 \\
\hline 6 & -1 & 6 & 1 & - & - & 0 & 0 & 0 \\
\hline 15 & 0 & 8 & 3 & 4 & 1 & 0 & 0 & 0 \\
\hline 1 & 1 & 2 & 2 & 1 & 4 & 0 & 0 & 0 \\
\hline
\end{tabular}

(g) All other occurrences of link invalidation at various nodes of various flows follow the same procedure as node 3 above, and ultimately some SBR/PHC-informed routes are chosen for all flows as follows:

Flow $1: 1 \rightarrow 2 \rightarrow 3 \rightarrow 8 \rightarrow 12 \rightarrow 15$

Flow $2: 3 \rightarrow 8 \rightarrow 12 \rightarrow 15 \rightarrow 14 \rightarrow 13$

Flow $3: 2 \rightarrow 6 \rightarrow 10 \rightarrow 14$

Flow $4: 16 \rightarrow 12 \rightarrow 8 \rightarrow 3 \rightarrow 2 \rightarrow 1$

Flow $5: 13 \rightarrow 10 \rightarrow 6 \rightarrow 3 \rightarrow 4$

Flow $6: 14 \rightarrow 10 \rightarrow 6 \rightarrow 3$ 
Note that link invalidations still continue to occur from time to time, and occasionally these routes might change. But they always tend to revert back to a combination that presents the best SBR/PHC measure of the moment given the beams used by all nodes at that moment.

\subsection{Summary}

MBA-DRR is primarily based on AODV and RGR. The general inner-working at the high-level is the same. However, there is a fundamental difference in a key detail: MBA-DRR chooses routes not based on the shortest-path criteria, but rather on a more-stars-less-bridges preference basis. This shift is motivated by the fact that MBADRR specifically and ultimately targets the reduction of the E2E delay in multi-flow scenarios. MBA-DRR is proposed to take advantage of MBA capabilities to reduce E2E delay. The preference on more-stars-less-bridges is dictated by preliminary work results that have shown that: a) the shortest-path routes usually have a relatively high E2E delay in multi-flow scenarios, and b) when minimal E2E delay is attained, the routes feature a lot of star nodes and very few to none bridges. In order to apply a more-stars-less-bridges preference approach, MBA-DRR continuously propagates (through control packets) information about the number of stars and bridges along discovered routes. To use this information for the ranking of the routes, we define two metrics: SBR and PHC. The SBR metric is defined as the number of stars over the number of bridges. To define PHC, we see the rank of a flow in a bridge as an additional hop that that flow goes through to account for the delay spent while waiting for its turn to occupy the beam/link. Finally, we define an SBR/PHC measure that helps us compare different routes with the goal of choosing the one that facilitates lower delay for all the flows. 


\section{Chapter 7}

\section{Evaluation of the MBA-DRR Routing Protocol}

\subsection{Introduction}

In this chapter, we evaluate the MBA-DRR protocol in various scenarios. We show that MBA-DRR achieves our goal of reducing the E2E delay in multi-flow networks. We conduct this evaluation in three main sets. First, in a static scenario, we show how the routes selected by MBA-DRR compare to the ones suggested by the optimization model. We also make a comparison to the routes selected by RGR, a shortest-routebased protocol. Secondly, in a mobile scenario, we show how MBA-DRR, running over a suitable multi-beam MAC, is advantageous over running RGR on omnidirectional MAC, single-beam MAC and multi-beam MAC. Finally, we further compare MBADRR and RGR, both running over the same multi-beam MAC, when the network size grows, when the traffic intensity grows, and when the beamwidth of the MBA varies. These comparisons/evaluations demonstrate the success of MBA-DRR in reducing E2E delay in multi-flow ad hoc networks, especially mobile ones. By default, and unless specified otherwise, we are arbitrarily going to use a beamwidth of $60^{\circ}$ for directional antennas and a packet generation rate of 5 pkts/sec. 


\subsection{Static Scenarios: Comparing Opimal Routes, MBA-DRR, and RGR}

In this section, we show how the MBA-DRR routing protocol compares to the solution found by the optimization model (Chapter 5), and to the shortest-route-based protocol RGR.

\subsubsection{Running-Example Static Scenario}

We perform the comparison in two different ways. In the first way, we first obtain the routes given by each protocol (Optimal Solution, MBA-DRR, and RGR) and then we use the optimization tool introduced in Chapter 5 to find the optimal scheduling of time slots for those routes. We obtain the delay in terms of time slots per flow for each protocol. In the second comparison, we simply run the protocols on the simulator (OPNET) and we collect the E2E delay in seconds. For the optimal routes, we hardcode the routing tables of the nodes as given by the optimal model.

\subsubsection{Comparison by Time Slot Scheduling}

In the 12-node static scenario (Figure 7.1) introduced in Section 5.3.2, the optimal routes chosen ${ }^{1}$ for the six flows are as follows:

Flow 1: $1 \rightarrow 6 \rightarrow 3 \rightarrow 8 \rightarrow 12 \rightarrow 15$

Flow 2: $3 \rightarrow 6 \rightarrow 10 \rightarrow 13$

Flow $3: 2 \rightarrow 6 \rightarrow 10 \rightarrow 14$

Flow $4: 16 \rightarrow 15 \rightarrow 10 \rightarrow 6 \rightarrow 1$

Flow $5: 13 \rightarrow 10 \rightarrow 15 \rightarrow 12 \rightarrow 8 \rightarrow 4$

Flow $6: 14 \rightarrow 15 \rightarrow 12 \rightarrow 8 \rightarrow 3$

The routes chosen by MBA-DRR with the same scenario are as follows:

Flow $1: 1 \rightarrow 2 \rightarrow 3 \rightarrow 8 \rightarrow 12 \rightarrow 15$

Flow $2: 3 \rightarrow 6 \rightarrow 10 \rightarrow 13$

Flow $3: 2 \rightarrow 3 \rightarrow 8 \rightarrow 12 \rightarrow 15 \rightarrow 14$

\footnotetext{
${ }^{1}$ These optimal routes are depicted in Figure 5.3 in Chapter 5
} 
Flow $4: 16 \rightarrow 12 \rightarrow 8 \rightarrow 3 \rightarrow 2 \rightarrow 1$

Flow $5: 13 \rightarrow 10 \rightarrow 6 \rightarrow 3 \rightarrow 4$

Flow $6: 14 \rightarrow 10 \rightarrow 6 \rightarrow 3$

The routes chosen by RGR with the same scenario are as follows:

Flow $1: 1 \rightarrow 6 \rightarrow 10 \rightarrow 15$

Flow 2: $3 \rightarrow 6 \rightarrow 10 \rightarrow 13$

Flow 3: $2 \rightarrow 6 \rightarrow 10 \rightarrow 14$

Flow $4: 16 \rightarrow 15 \rightarrow 10 \rightarrow 6 \rightarrow 1$

Flow $5: 13 \rightarrow 10 \rightarrow 6 \rightarrow 3 \rightarrow 4$

Flow $6: 14 \rightarrow 10 \rightarrow 6 \rightarrow 3$

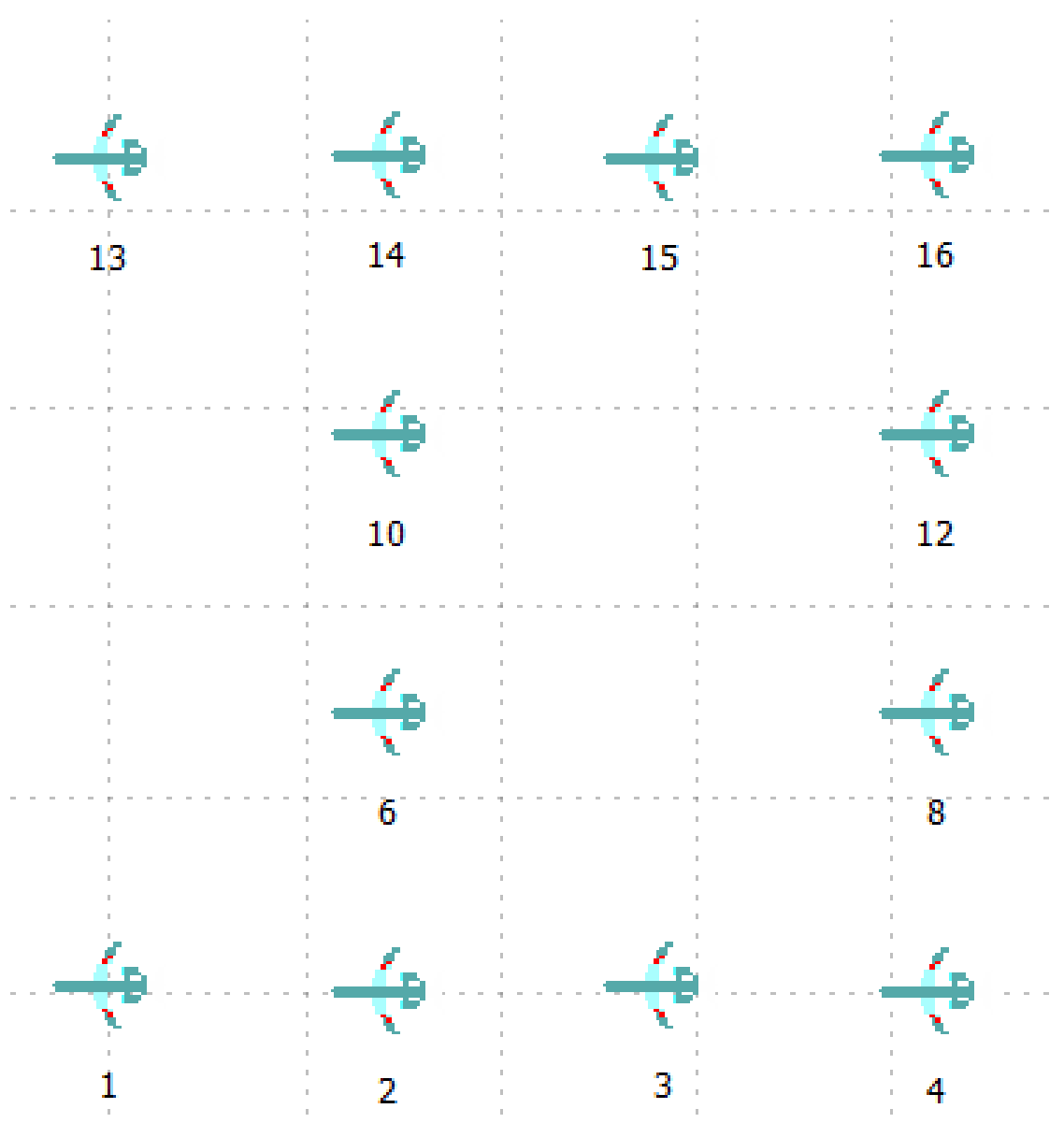

Figure 7.1: Static Scenario 
In the case of the optimal routes, the time slots' scheduling is presented in Table 7.1 (as established in Table 5.10 in Section 5.4.2), yielding an optimal end-to-end delay of 4.5 time slots per flow on average. With the routes chosen by MBA-DRR, the optimization tool gives an optimal scheduling of the time slots as given in Table 7.2, which gives an average end-to-end delay of $4.8 \overline{3}$ time slots per flow. This is a $7 \%$ increase in delay compared to the optimal routes.

Table 7.1: Optimal Link Scheduling for Optimal Routes

\begin{tabular}{|c|c|c|c|c|c|c|c|}
\hline Traffic flow & slot 1 & slot 2 & slot 3 & slot 4 & slot 5 & slot $6 \quad$ slot 7 & slot 8 \\
\hline Flow 1 & $(1,6)$ & $(6,3)$ & $(3,8)$ & $(8,12)$ & $(12,15)$ & & \\
\hline Flow 2 & & & $(3,6)$ & $(6,10)$ & $(10,13)$ & & \\
\hline Flow 3 & $(2,6)$ & $(6,10)$ & $(10,14)$ & & & & \\
\hline Flow 4 & $(16,15)$ & $(15,10)$ & $(10,6)$ & $(6,1)$ & & & \\
\hline Flow 5 & $(13,10)$ & & $(10,15)$ & $(15,12)$ & $(12,8)$ & $(8,4)$ & \\
\hline Flow 6 & $(14,15)$ & $(15,12)$ & $(12,8)$ & $(8,3)$ & & & \\
\hline
\end{tabular}

Table 7.2: Optimal Link Scheduling for MBA-DRR Routes

\begin{tabular}{|c|c|c|c|c|c|c|c|c|}
\hline Traffic flow & slot 1 & slot 2 & slot 3 & slot 4 & slot 5 & slot 6 & slot 7 & slot 8 \\
\hline Flow 1 & & $(1,2)$ & $(2,3)$ & $(3,8)$ & $(8,12)$ & $(12,15)$ & & \\
\hline Flow 2 & & $(3,6)$ & $(6,10)$ & $(10,13)$ & & & & \\
\hline Flow 3 & $(2,3)$ & $(3,8)$ & $(8,12)$ & $(12,15)$ & $(15,14)$ & & & \\
\hline Flow 4 & $(16,12)$ & $(12,8)$ & $(8,3)$ & $(3,2)$ & $(2,1)$ & & & \\
\hline Flow 5 & $(13,10)$ & $(10,6)$ & $(6,3)$ & $(3,4)$ & & & & \\
\hline Flow 6 & & & $(14,10)$ & $(10,6)$ & $(6,3)$ & & & \\
\hline
\end{tabular}

With the routes chosen by RGR, a shortest-route-based protocol, the optimization tool provides an optimal scheduling of the time slots as shown in Table 7.3, for an average end-to-end delay of $5.6 \overline{6}$ time slots per flow. This is a $26 \%$ increase in delay compared to the optimal routes, and a $17 \%$ increase in delay compared to MBA-DRR. 
Table 7.3: Optimal Link Scheduling for RGR Routes

\begin{tabular}{lllllllll}
\hline Traffic flow & slot 1 & slot 2 & slot 3 & slot 4 & slot 5 & slot 6 & slot 7 & slot 8 \\
\hline \hline Flow 1 & $(1,6)$ & & & & & & $(6,10)$ & $(10,15)$ \\
Flow 2 & & $(3,6)$ & & & $(6,10)$ & $(10,13)$ & & \\
Flow 3 & $(2,6)$ & & $(6,10)$ & $(10,14)$ & & & & \\
Flow 4 & $(16,15)$ & & $(15,10)$ & $(10,6)$ & $(6,1)$ & & & \\
Flow 5 & $(13,10)$ & $(10,6)$ & $(6,3)$ & $(3,4)$ & & & & \\
Flow 6 & & & $(14,10)$ & & & $(10,6)$ & $(6,3)$ & \\
\hline
\end{tabular}

As we can see, MBA-DRR chooses routes that are different than the absolute optimum; however, the routes chosen by MBA-DRR are closer to the optimum in terms of the resulting end-to-end delay than the routes chosen by RGR, the shortest-route-based protocol. In fact, they are also closer in terms of the overall star-to-bridge ratio. In effect, by examining Table 7.1, we find that there are 5 star nodes formed and 3 bridges, for an overall SBR of $5 / 3=1.6 \overline{6}$. The stars and bridges are identified as follows. The first star is node 6 between slot 1 and slot 2 . Two flows (flow 1 and flow 3 ) are incoming to node 6 at slot 1, and the same flows are outgoing from node 6 in the following slot (slot 2). This is the very definition of a star; a node servicing two or more flows in two consecutive slots. Similar observations result in determining that: node 10 is a star between slot 2 and slot 3 , node 8 is a star between slot 3 and slot 4, node 6 again is a star between slot 3 and slot 4, node 12 is a star between slot 4 and slot 5 . For the bridges, a quick way to count them is to count the gaps in the table. A gap means that the transmission had to wait because another flow was occupying the link (the beam). In Table 7.1, there are 3 such gaps: 2 for flow 2 in slot 1 and slot 2 , and 1 for flow 5 in slot 2 . A similar analysis on Table 7.2 shows that MBA-DRR's routes feature 4 star nodes formed and 4 bridges, for an overall SBR of $4 / 4=1$. Finally, an analysis on Table 7.3 shows 2 star nodes and 14 bridges, for an overall SBR of $2 / 14=0.14$. We can clearly see that the optimal route selection has the highest SBR $(1.6 \overline{6})$, followed by MBA-DRR (1). RGR comes far behind with an SBR of just 0.14.

The difference between the optimal routes and the routes chosen by MBA-DRR is explained as follows. The optimization tool, when computing the optimal routes 
to choose, considers all possible routes. However, as pointed out in Section 6.6, when the protocol is running, practical/physical limitations result in the fact that not all possible routes are considered in the selection. These practical limitations include: rx-mode/tx-mode switching, medium contention, and collisions. For instance, because a node is switched to tx-mode, it can miss a given RREQ (which is a broadcast packet, hence not retransmitted unless a route is not found in the end), resulting in at least one route that will not be discovered. In the same vein, medium contention and collisions prevent some packets from being transmitted/received. Because of these, some RREQ/RREP will be lost and some routes will not be discovered.

In the end, the results shown above meet our expectation that was also stated in Section 6.6. In effect, the philosophy of star/bridge, when followed, albeit on a best-effort basis, proves to result in a significant improvement in latency compared to a shortest-route-based protocol.

\subsubsection{Comparison in Simulator}

When we run RGR, MBA-DRR and the optimal routes in OPNET, the results are as shown in Figure 7.2. The time-averaged delay is steadier for all the protocols toward the end of the 30-minute simulation; therefore, these are the values that we consider. The optimal routes give a delay of $2.5 \mathrm{~ms}$. MBA-DRR gives a delay of 4.4 ms. This is a $1.9 \mathrm{~ms}$ increase compared to the optimal-route case. RGR gives a delay of $48 \mathrm{~ms}$. This is a $45.5 \mathrm{~ms}$ increase compared to the optimal routes. The results shows that MBA-DRR considerably cuts down the end-to-end delay of RGR in this static scenario. The MBA-DRR delay is closer to the optimal solution than RGR, something that was already shown in the previous section as well. There is a spike observed toward the beginning of the simulation for RGR. This is due to the fact that, because it is shortest-route based, RGR is inherently prone to bridges (queues). At some that point toward the beginning of the simulation, there was a bit of a burst in the generated traffic ${ }^{2}$, and this compounded the effects of the queuing. Nevertheless, as already mentioned, we only consider the values toward the end of the simulation, as they become steadier.

\footnotetext{
${ }^{2}$ The traffic generation is not constant; rather it follows an exponential distribution.
} 


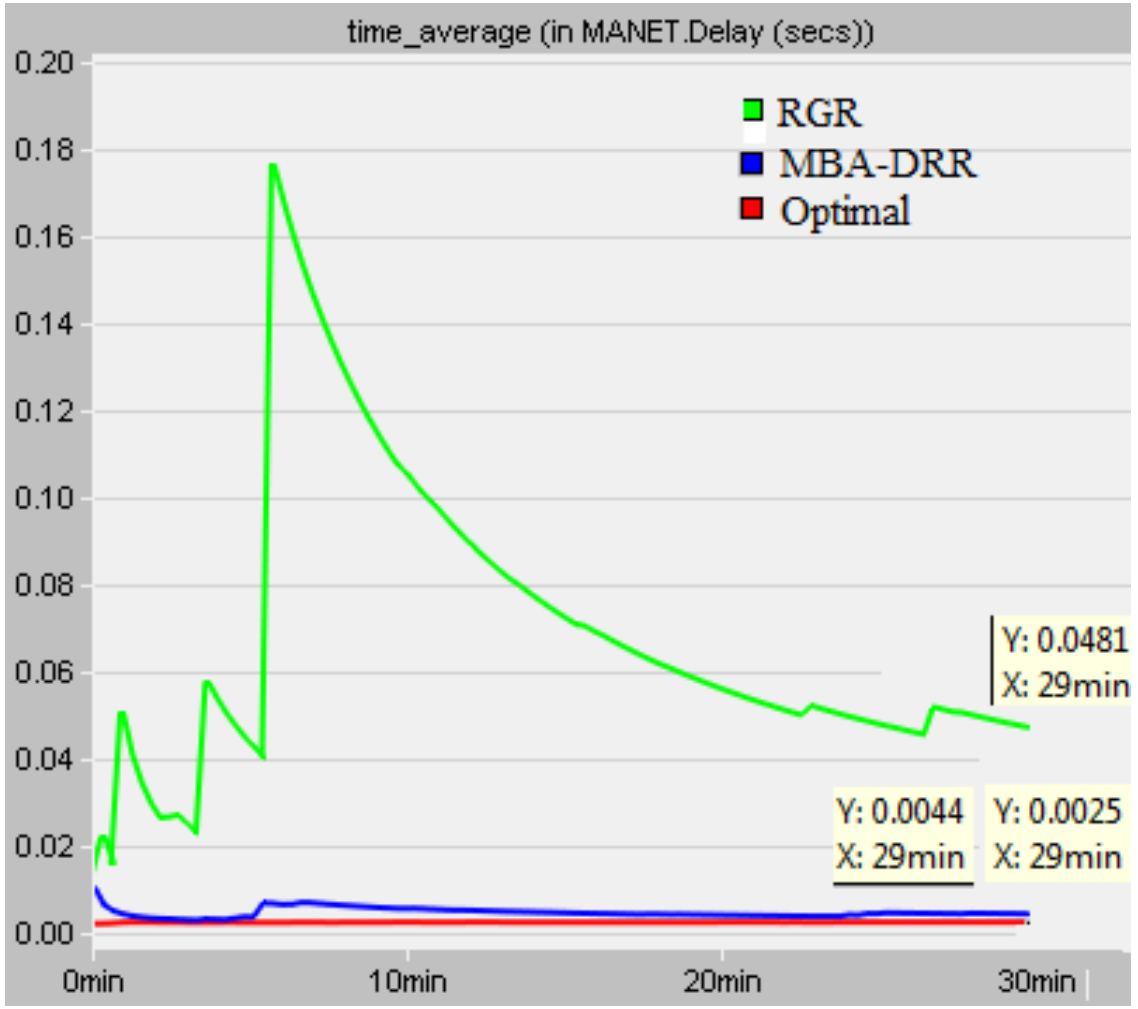

Figure 7.2: Delay: Optimal vs MBA-DRR vs RGR

\subsubsection{Random Static Scenarios}

To further explain the difference in delay between the optimal solution, MBA-DRR, and RGR, we run ten smaller random 10-node 5-flow static scenarios. The scenarios are depicted in Figure B.1 through Figure B.10 in Appendix B. The optimal route selection and scheduling (as given by the optimizer) for each scenario is given in Table B.1 through Table B.10 in Appendix B. Based on these optimal route selections and scheduling, the path length for each scenario and the average end-to-end delay in terms of time slots are summarized in Table 7.4. Based on this table, the average path length across all 10 scenarios is 2.34 hops, and the average end-to-end delay is 2.66 slots. This can be re-stated as follows: the optimal path has 2.34 hops but packet delivery takes 2.66 time slots; therefore, the difference 2.66-2.34=0.32 indicates how often, on average, a packet is queued/delayed in the optimal solution.

Using the same ten scenarios, we run MBA-DRR and RGR in OPNET. For each 
Table 7.4: Path Length and Delay for Optimal Link Scheduling

\begin{tabular}{cccccccccccc}
\hline Scenario & 1 & 2 & 3 & 4 & 5 & 6 & 7 & 8 & 9 & 10 & Average \\
\hline \hline Average Path Length (in hops) & 3 & 2.2 & 2 & 2.4 & 2 & 2.6 & 2.2 & 2.2 & 1.8 & 3 & $\mathbf{2 . 3 4}$ \\
Average E2E Delay (in time slots) & 3.4 & 2.4 & 2.2 & 2.8 & 2.2 & 2.8 & 2.6 & 2.8 & 2.2 & 3.2 & $\mathbf{2 . 6 6}$ \\
\hline
\end{tabular}

scenario, we collect both the average path length and the average number of times ${ }^{3}$ that a packet joins a (beam-specific) queue. These results are presented in Table 7.5 and Table 7.6 for MBA-DRR and RGR respectively.

Table 7.5: Path Length and Number of Queuings for MBA-DRR

\begin{tabular}{cccccccccccc}
\hline Scenario & 1 & 2 & 3 & 4 & 5 & 6 & 7 & 8 & 9 & 10 & Average \\
\hline \hline Average Path Length (in hops) & 1.8 & 2 & 2.6 & 2.2 & 2 & 2.4 & 2 & 2 & 2.2 & 2 & $\mathbf{2 . 1 2}$ \\
Average Number of Queuings & 0.36 & 0.49 & 1.2 & 1.2 & 0.72 & 0.45 & 0.45 & 0.36 & 1.03 & 0.64 & $\mathbf{0 . 6 9}$ \\
\hline
\end{tabular}

Table 7.6: Path Length and Number of Queuings for RGR

\begin{tabular}{cccccccccccc}
\hline Scenario & 1 & 2 & 3 & 4 & 5 & 6 & 7 & 8 & 9 & 10 & Average \\
\hline \hline Average Path Length (in hops) & 1.8 & 1.6 & 2 & 1.6 & 2 & 3 & 2 & 2 & 2 & 2.2 & $\mathbf{2 . 0 2}$ \\
Average Number of Queuings & 1 & 1.63 & 0.46 & 1.11 & 0.84 & 0.85 & 0.17 & 0.95 & 0.70 & 1.15 & $\mathbf{0 . 8 9}$ \\
\hline
\end{tabular}

Based on these tables/results, for MBA-DRR, the average path length across all 10 scenarios is 2.12 hops, and the average number of queuings is 0.69 . Likewise, for RGR, the average path length across all 10 scenarios is 2.02 hops, and the average number of queuings is 0.89 .

These results show that, with the ten random static scenarios, the queuing is least with the optimal solution (0.32), followed by MBA-DRR (0.69). RGR exhibits the worst queuing (0.89). This ranking is the opposite for the average path length: it is longest with the optimal solution (2.34), followed by MBA-DRR (2.12). RGR exhibits the shortest average path length (2.02).

\footnotetext{
${ }^{3}$ Whenever a high-layer packet finds a non-empty queue at the MAC level, this number is incremented by 1 . The average is computed over all the packets delivered over 1800 seconds of simulation.
} 
These results further show that, in order to minimize the E2E delay, the routes that are chosen are not necessarily the shortest. The route selection has to follow other criteria (such as bridge/star as shown in Chapter 5). In fact, a protocol that chooses its routes to be the shortest ends up incurring more packets waiting in the queues, hence a higher E2E delay. Conversely, a protocol such as MBA-DRR that tries, at best-effort, to emulate the bridge/star selection criteria significantly reduces the amount of packets waiting in queues, hence shortening the E2E delay.

\subsection{Mobile Scenario: Comparing MBA-DRR, RGR, and AODV}

With a mobile scenario, we show in this section how different types of antenna + protocol combinations fare. Namely, we evaluate: Omnidirectional antenna + RGR (RGR-Omni), Single-beam directional antenna + RGR (RGR-SBA), Multi-beam directional antenna + RGR (RGR-MBA, and MBA + MBA-DRR (MBADRR-MBA).

As exlained earlier, RGR is a routing protocol that selects routes based on shortest distance (hop count), whereas MBA-DRR departs from that central criterion. Each antenna type works with an appropriate MAC protocol. We simply run the scenarios and measure the E2E delay and the PDR. The goal here is to show how E2E delay keeps improving drastically as we shift from omnidirectional antenna to MBA, with specifically designed MAC and routing protocols for the latter. We show that there are major gains to be had at none or negligible expense in PDR.

For this evaluation purpose, we create a 30-node and 15-flow mobile scenario where all the nodes move at speeds that vary from [50-60] m/s. This speed range is chosen according to medium-sized UAV speed ranges presented in [108]. The high mobility of some MANETs, such as FANETs, causes intermittent and episodic connections which brings unique challenges such as frequently changed topologies [109]. We generate ten independent runs using ten different seeds of the pseudo-random number generator available in OPNET. By doing so, we have ten sets of pseudo-independent results. These ten results are then averaged and the 95\% confidence intervals determined and shown in the figures (as vertical lines). These confidence intervals serve to establish the statistical significance of the difference 
between any two plots (results). The difference between the plots is statistically significant if the vertical lines representing the confidence intervals do not overlap.

Figure 7.3 shows that the E2E delay when using omnidirectional antennas is very high compared to when directional antennas are used. This clearly shows that omnidirectional antennas ought to be avoided altogether in a multi-flow scenario where time (in terms of delay) is of the essence. In effect, omnidirectional antennas yield a delay of $700 \mathrm{~ms}$ compared to a delay in the 10's of milliseconds for directional antennas. It can be seen that the E2E delay continues to increase for the Omni-MANET. This is because of severe queuing and a combination of other issues such as exposed-terminal that cause many transmissions to have to be retried, hence accumulating delay. And this situation keeps worsening as the simulation progresses. Therefore, the mere fact of using a directional antenna (be it single-beam or multi-beam) slashes the E2E delay in such mobile multi-flow scenario. By simply switching from an omnidirectional antenna to a single-beam antenna, while running the same routing protocol (RGR), the E2E delay is significantly reduced from 700 $\mathrm{ms}$ to $40 \mathrm{~ms}$. Recall that we need a MAC protocol to run the single-beam antenna. As such, we proposed a MAC protocol (DbMAC) in Chapter 2.

A closer look at the results for directional antennas (Figure 7.4) shows interesting outcomes. First, by switching from a single-beam antenna to a multi-beam one, still while running the same RGR routing protocol, the E2E delay is further cut in half from $40 \mathrm{~ms}$ to $20 \mathrm{~ms}$. Recall that we need a MAC protocol to run the multi-beam antenna. We proposed such a MAC protocol (MBA-DbMAC) in Chapter 4.

Secondly, with the multi-beam antenna, by switching from RGR to MBA-DRR (Proposed in Chapter 6), a routing protocol tailored for MBA and delay reduction, the E2E delay is yet again further cut in a little more than half from $20 \mathrm{~ms}$ to $9 \mathrm{~ms}$. Having a smarter routing protocol that exploits the capabilities of MBAs shows to pay off. It is worth noting that all these gains in E2E delay come at virtually no expense in the PDR, as Figure 7.5 suggests. This means that all the packets delivered with omnidirectional antennas and shortest-route-based routing protocol are still delivered with multi-beam directional antennas and our smarter MBA-based 


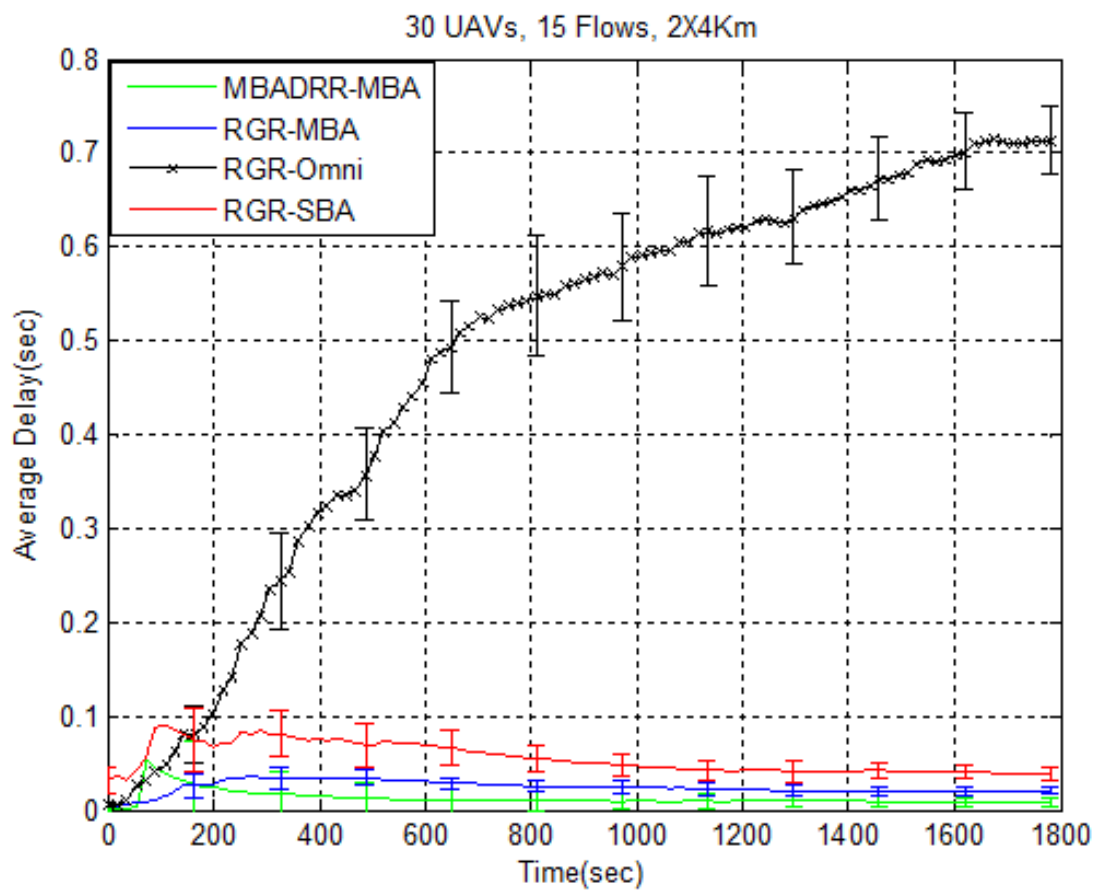

Figure 7.3: Delay, 30-Node Mobile Scenario

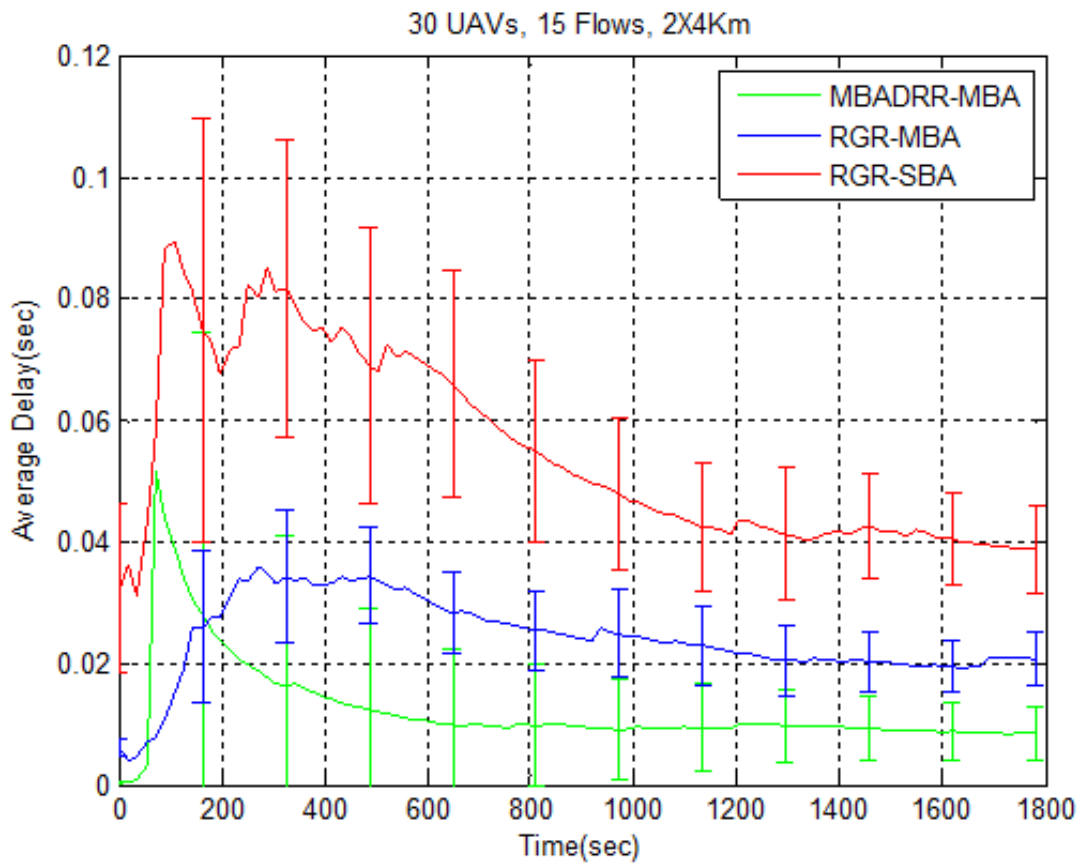

Figure 7.4: Delay, 30-Node Mobile Scenario, Without Omni 
routing protocol, but in considerably less time. Unfortunately, we note that, in the interim step of switching from an omnidirectional antenna to a single-beam antenna, a statistically significant drop in PDR occurs due to the adverse effect of deafness (as pointed out in Chapter 4). But this is not a concern to dwell on because the introduction and full exploitation of MBAs does "recover" the "lost" PDR while further reducing the E2E delay.

As Figure 7.6 suggests, the gain in delay with MBA-DRR comes at the expense of a considerably higher control overhead. The control overhead or routing overhead is simply the sum of RREQs, RREPs, RERRs, and HELLO messages per second. This increase in overhead can be explained by the fact that MBA-DRR, in the quest to discover as many potential routes as possible, allows the propagation of RREQs and RREPs more than RGR does. For example, as noted in Sections 6.6 and 6.8.3, RGR does not allow an RREQ with the same ID and originator to be processed twice by a given node. We know that, in the context of MBAs, an RREQ with the same ID and originator might have taken a completely different route than the previously received one. Therefore, MBA-DRR does allow a node to process an RREQ with the same ID and originator as a previously received one; as long as it has not gone through the current node before. The limitations on RREQs introduced by the concepts of safe-distance and scoped-flooding of RGR are also removed. All these relaxations cause the significantly higher routing overhead. We assume other costs (such as the cost to switch modes and the cost to select which antenna sector to transmit a packet on) to be negligible, and we do not consider them in these simulation results.

The results above are of utmost significance. In fact, they are central to our work overall. We have been able to successfully reduce the E2E delay of a mobile multi-flow network of UAVs. We have been able to bring this delay from $700 \mathrm{~ms}$ down to just $9 \mathrm{~ms}$. This has been done in three major steps: a) a shift from omnidirectional antenna to single-beam directional antenna by means of selection of a new antenna type and the design of a MAC protocol to work with it; b) a shift from single-beam directional antenna to multi-beam directional antenna by means of selection of a new antenna type and the design yet again of a MAC protocol to work with it; and finally c) a departure from the widespread shortest-route selection philosophy to a 


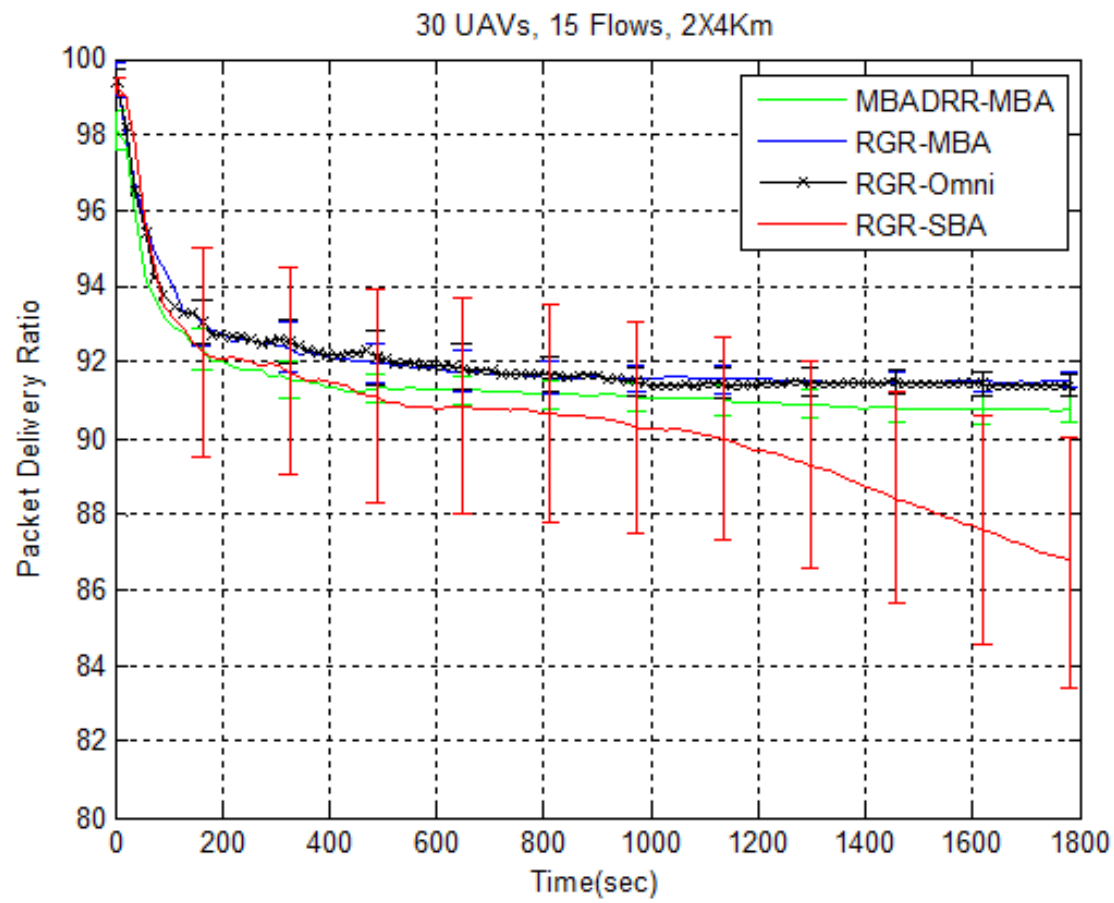

Figure 7.5: PDR, 30-Node Mobile Scenario

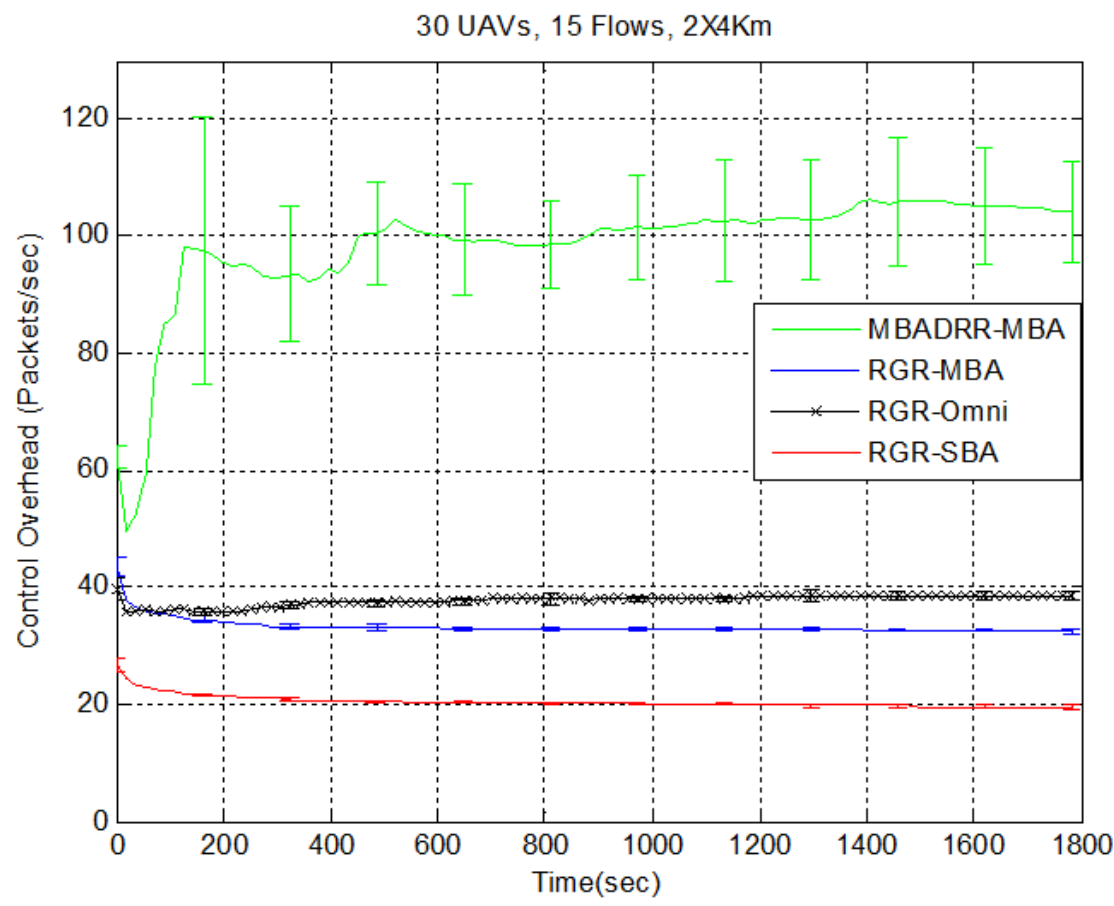

Figure 7.6: Control Overhead, 30-Node Mobile Scenario 
smarter route selection philosophy that exploits the benefits of multi-beam functionality with the concepts of stars and bridges in the design of a new routing protocol.

On a side note, and for comparison purposes, Figure 7.7 shows the delay comparison when running AODV, a widespread MANET routing protocol that is also shortest-route-based. The delay of AODV over omnidirectional antennas is almost identical to that of RGR, at somewhat above $700 \mathrm{~ms}$. When single-beam antennas are used, AODV exhibits a delay in the $60 \mathrm{~ms}$ (worse than the $40 \mathrm{~ms}$ for RGR). Moreover, when it comes to MBAs, AODV has a delay in the $200 \mathrm{~ms}$. This calls for two observations: a) AODV fares worse than RGR again, and b) AODV is actually better off running over single-beam antennas than over multi-beam antennas. Recall that neither AODV nor RGR was designed with MBA capabilities in mind. Our second observation is that, contrary to RGR, AODV is actually negatively affected by "multi-beaming". This is a bit surprising and warrants a further investigation that will be part of our future work. Overall though, the use of directional antennas (single-beam or multi-beam) is still considerably better than the use of omnidirectional antennas in multi-flow mobile scenarios. The PDR comparison is shown in Figure 7.8. It is worth pointing out that MBA-DRR on MBA has by far the highest PDR over all AODV combinations; which is expected since MBA-DRR is based on RGR, which our previous work [8] has shown to outperform AODV in terms of PDR.

\subsection{Further Parameterized Evaluation of MBA- DRR}

In this section, we further evaluate how MBA-DRR performs in mobile scenarios as certain simulation parameters vary. Specifically, we vary: the network size, the traffic intensity, and the beamwidth. We focus the comparison on the performance of MBADRR against that of RGR, running on MBA as well. RGR running on MBAs was the combination that was the closest in performance in the previous evaluation. We have already shown the superiority of MBAs over SBAs and omnidirectional antennas in the previous evaluation. 


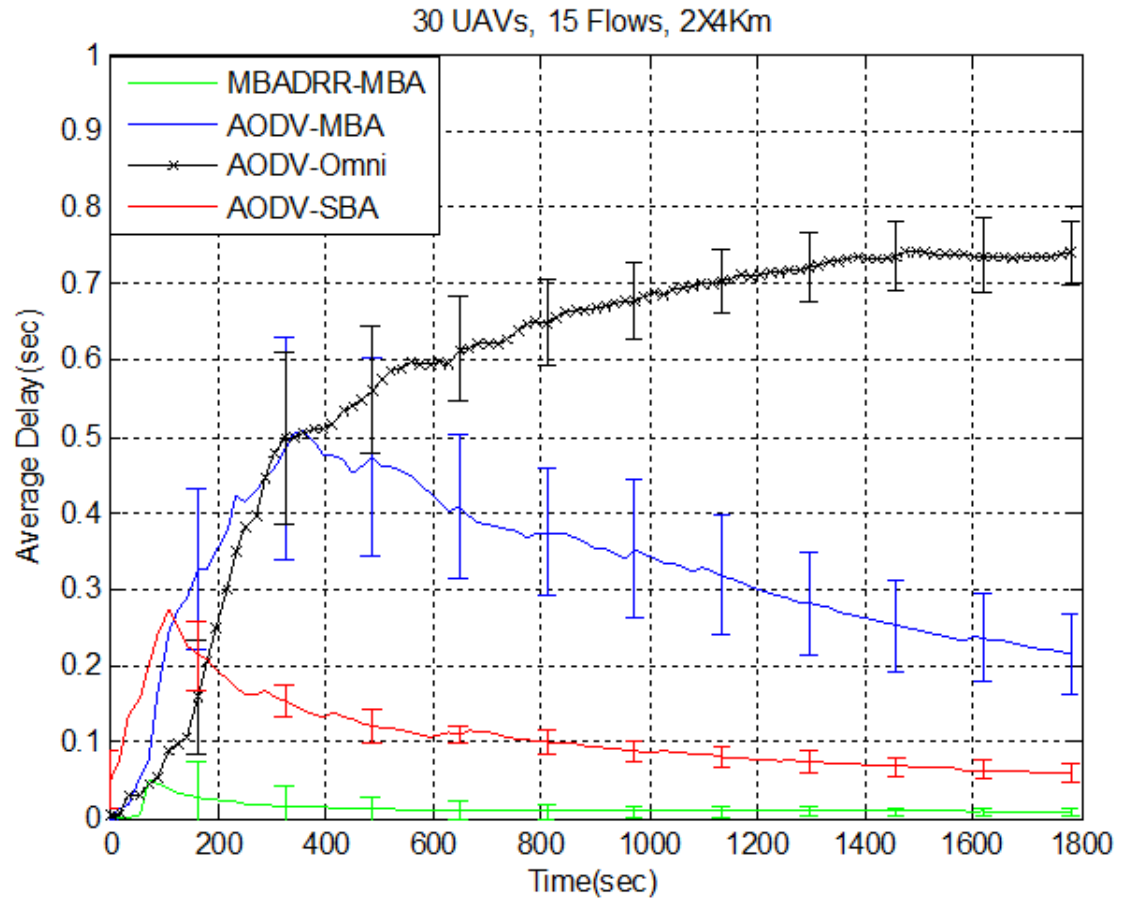

Figure 7.7: Delay, 30-Node Mobile Scenario, Comparison with AODV

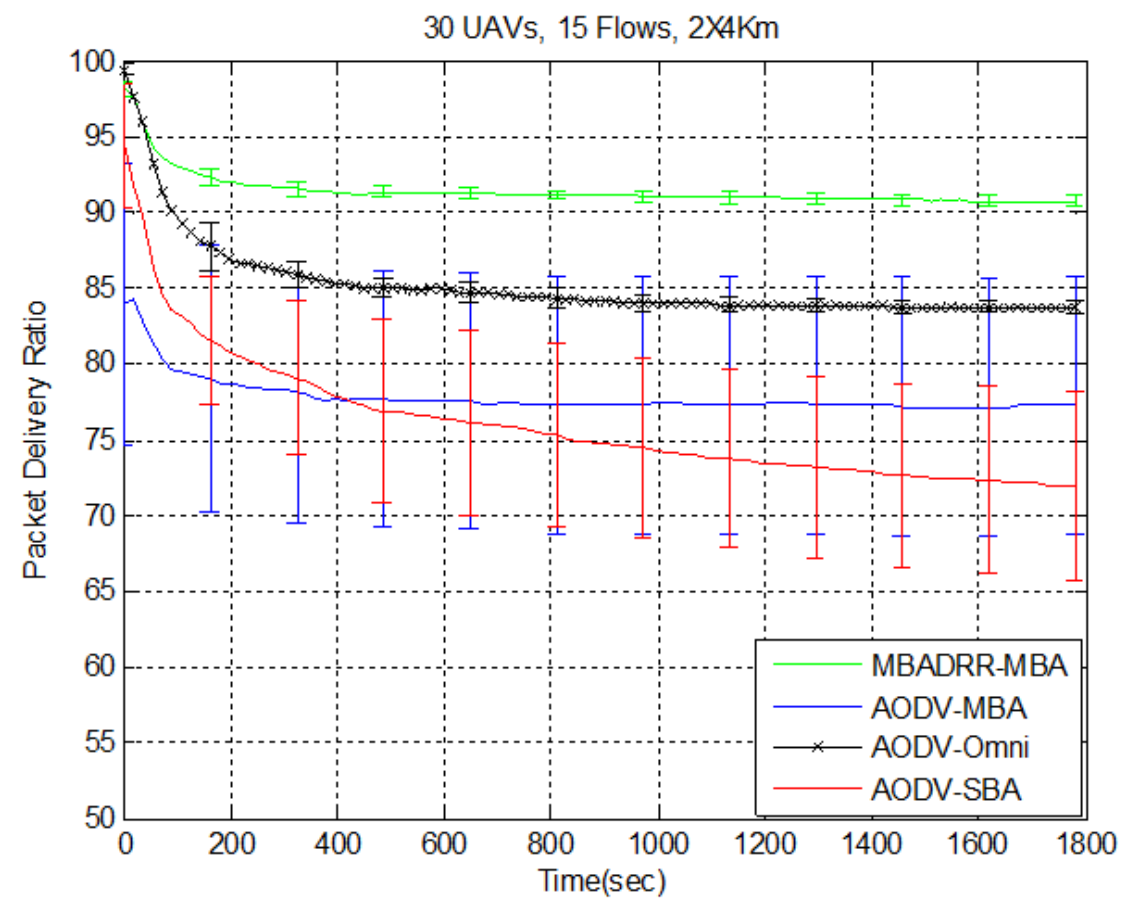

Figure 7.8: PDR, 30-Node Mobile Scenario, Comparison with AODV 


\subsubsection{Varying Network Size}

We vary the network size while making sure to keep the same node density overall. We consider four network sizes: 10, 20, 25, and 30 nodes. For each network size, we choose the number of flows to be equal to half the number of nodes. To maintain the same node density that we previously had for the 30-node scenario in Section 7.3, we maintain the ratio area over number of nodes constant. Table 7.7 summarizes the dimensioning of the four networks.

Table 7.7: Network Size Dimensioning

\begin{tabular}{ccc}
\hline Number of Nodes & Number of Flows & Area $(\mathrm{m} \times \mathrm{m})$ \\
\hline \hline 10 & 5 & $2000 \times 1333.33$ \\
20 & 10 & $2000 \times 2666.66$ \\
25 & 12 & $2000 \times 3333.33$ \\
30 & 15 & $2000 \times 4000$ \\
\hline
\end{tabular}

Figure 7.9 shows the average E2E delay as the network size grows from 10 nodes to 30. It is apparent that MBA-DRR sustains its superiority in terms of E2E delay over RGR no matter the size of the multi-flow network. This comes as no surprise since Chapter 6 specifically designs MBA-DRR to exploit multi-beam antennas, something that is absent from RGR. The larger the network, the greater the gap between the two protocols. This is an interesting observation that speaks to the scalabilty of our routing solution. Notice, once again, that this E2E delay superiority comes at virtually no cost in terms of PDR as Figure 7.10 suggests.

\subsubsection{Varying Traffic Intensity}

In a network of 10 nodes and 5 flows, we vary the traffic intensity from 5 pkts/sec per flow to 100 pkts/sec per flow. Each packet has a mean size of 1024 Bytes. Table 7.8 summarizes the traffic intensity dimensioning. With 5 pkts/sec per flow, all the flows collectively supply a load of 1024 Byte/pkts $\times 8$ bits/Bytes $\times 5$ pkts $/$ sec $\times 5$ flows 


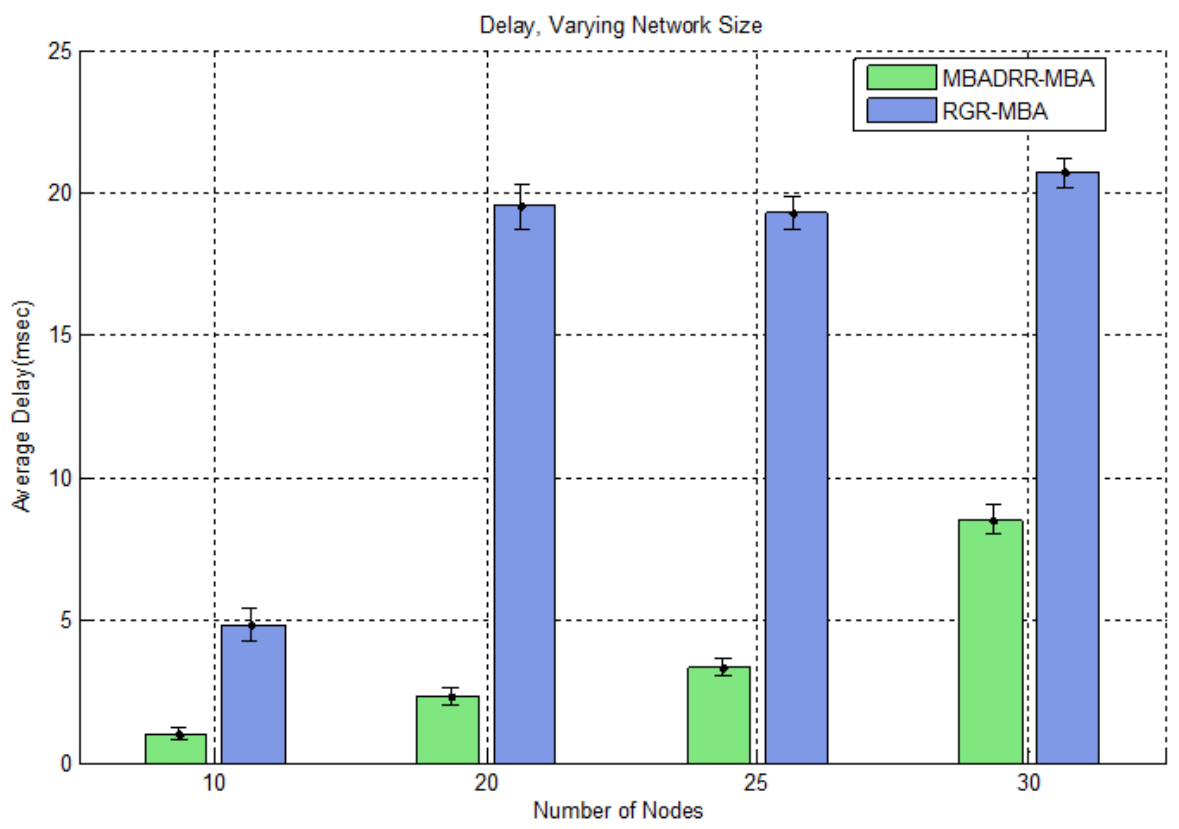

Figure 7.9: Delay, Mobile Scenario, Varying Network Size

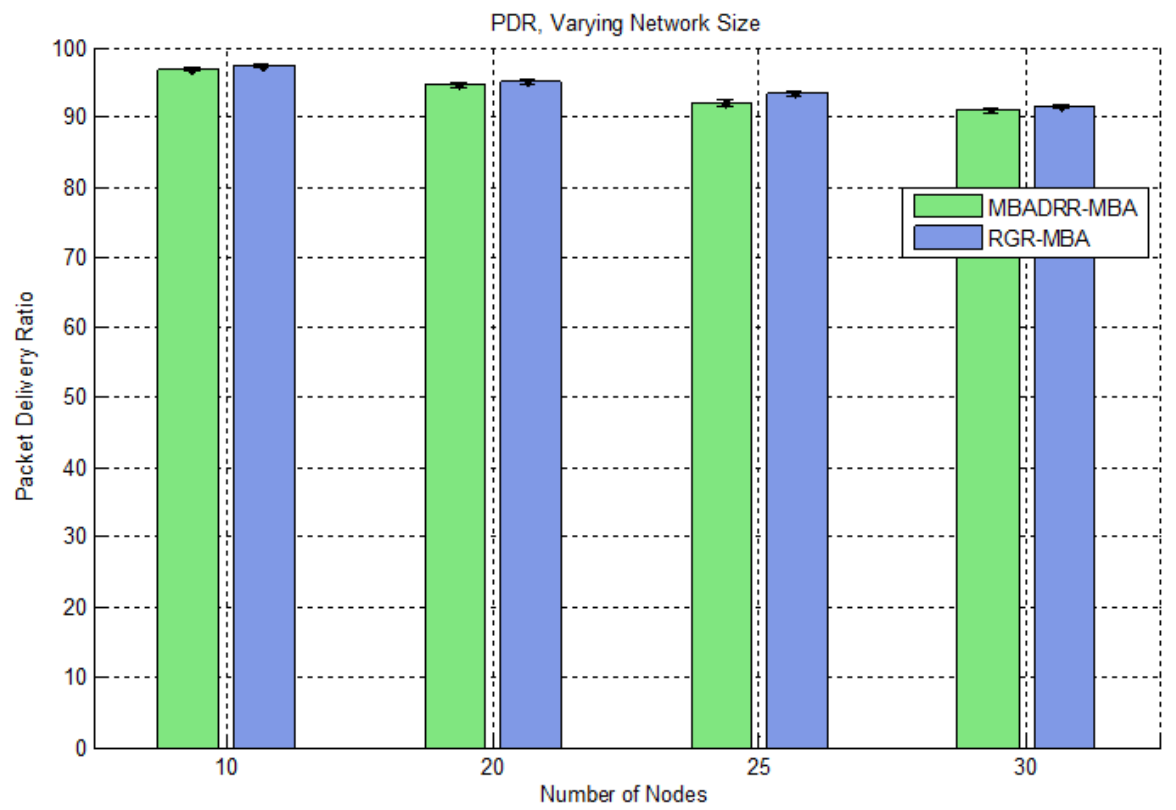

Figure 7.10: PDR, Mobile Scenario, Varying Network Size

$=204.8 \mathrm{Kbps}$; a load that is well below the channel nominal capacity of $11 \mathrm{Mbps}$. Likewise, with 100 pkts/sec per flow, all the flows collectively supply a load of 1024 $\times 8 \times 100 \times 5=4.096 \mathrm{Mbps}$. 
Table 7.8: Traffic Intensity Dimensioning in a 10-Node Network

\begin{tabular}{cc}
\hline Traffic Rate (pkts/sec) & Total Load (bps) \\
\hline \hline 5 & $204.8 \mathrm{~K}$ \\
50 & $2.048 \mathrm{M}$ \\
75 & $3.072 \mathrm{M}$ \\
100 & $4.096 \mathrm{M}$ \\
\hline
\end{tabular}

Figure 7.11 shows the average E2E delay as the network traffic intensity grows. Once again, we can clearly see that MBA-DRR sustains its superiority in terms of E2E delay over RGR no matter the traffic intensity of the multi-flow network. The increase in traffic intensity negatively affects the E2E delay of both protocols, but MBA-DRR maintains an almost constant reduction over RGR. Figure 7.12 shows that the E2E delay superiority is achieved at no cost in terms of PDR.

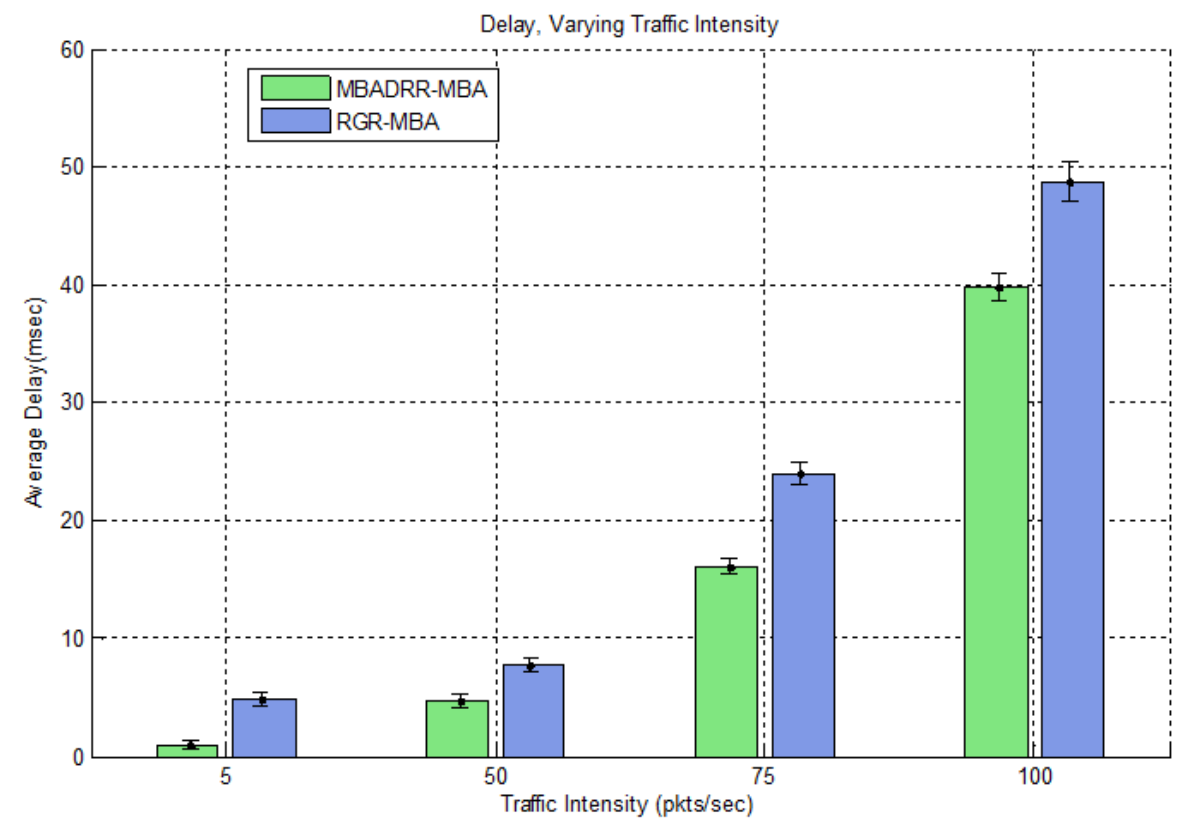

Figure 7.11: Delay, 10-Node Mobile Scenario, Varying Traffic Intensity 


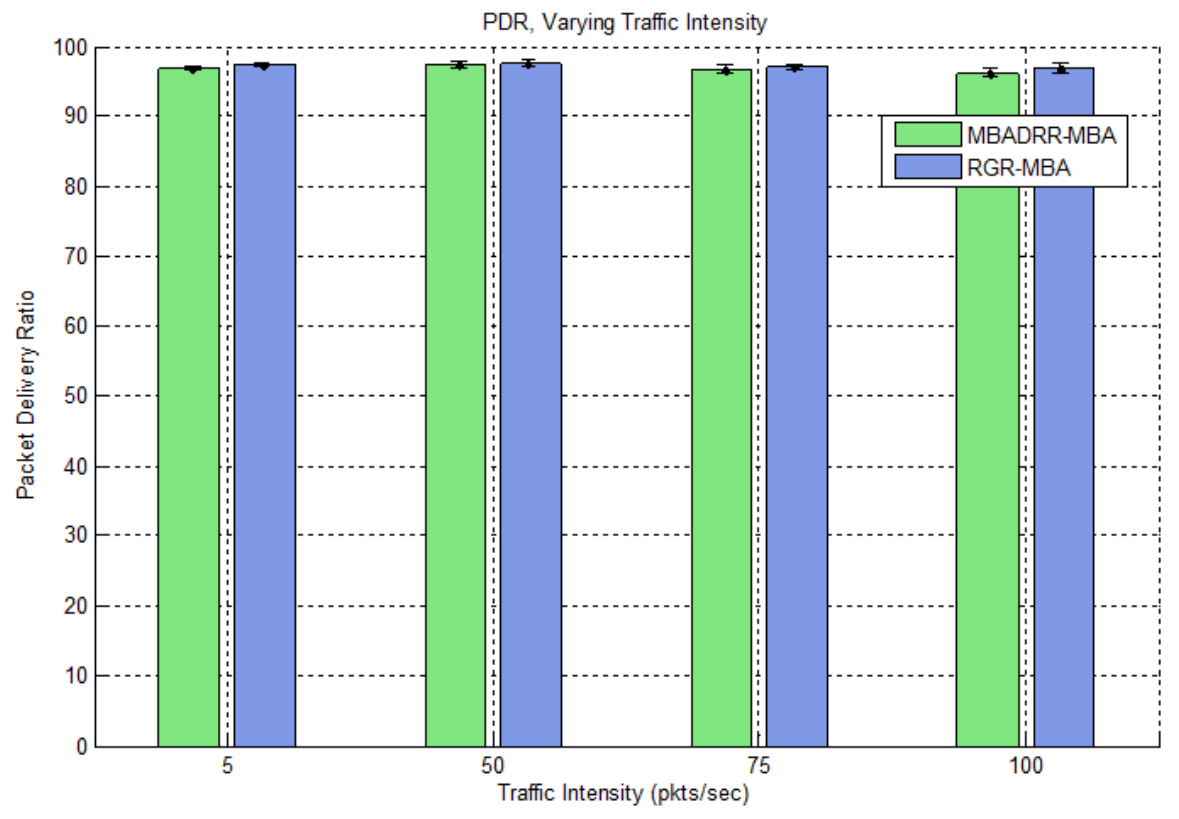

Figure 7.12: PDR, 10-Node Mobile Scenario, Varying Traffic Intensity

\subsubsection{Varying the Beamwidth}

In a network of 10 nodes and 5 flows, and a traffic intensity of 5 pkts/sec per flow, we vary the number of beams per antenna, hence the beamwidth of the antennas. The number of beams is inversely proportional to the beamwidth. For example, an MBA that has only 3 beams will have a beamwidth (per beam) of $360^{\circ} / 3=120^{\circ}$. We consider four different beam numbers: $3,4,6$, and 8 . Table 7.9 matches the considered numbers of beams with their corresponding beamwidths.

Table 7.9: Varying Beamwidth in a 10-Node Network

\begin{tabular}{cc}
\hline Number of beams & Beamwidth \\
\hline \hline 3 & $120^{\circ}$ \\
4 & $90^{\circ}$ \\
6 & $60^{\circ}$ \\
8 & $45^{\circ}$ \\
\hline
\end{tabular}

Figure 7.13 shows the average E2E delay as the number of beams (hence the beam width) grows. The superiority of MBA-DRR in terms of E2E delay over RGR 
is also maintained no matter the beamwidth of the antennas. It can be observed that the delay of RGR increases as the beamwidth decreases, further showing the inadequacy of RGR with MBAs. To the contrary, the delay stays almost constant with MBA-DRR. These observations are a bit counter-intuitive nevertheless and could be explained by the fact that 5 flows are not enough to actually make a considerable difference when the beamwidth is factored in. This vouches for another set of runs with a scenario that has more flows in order to see if there is a trend. And we do that next. Nevertheless, as it stands now, the take-away remains that MBA-DRR consistently outperforms RGR. Figure 7.14 shows no PDR expense for this E2E delay superiority.

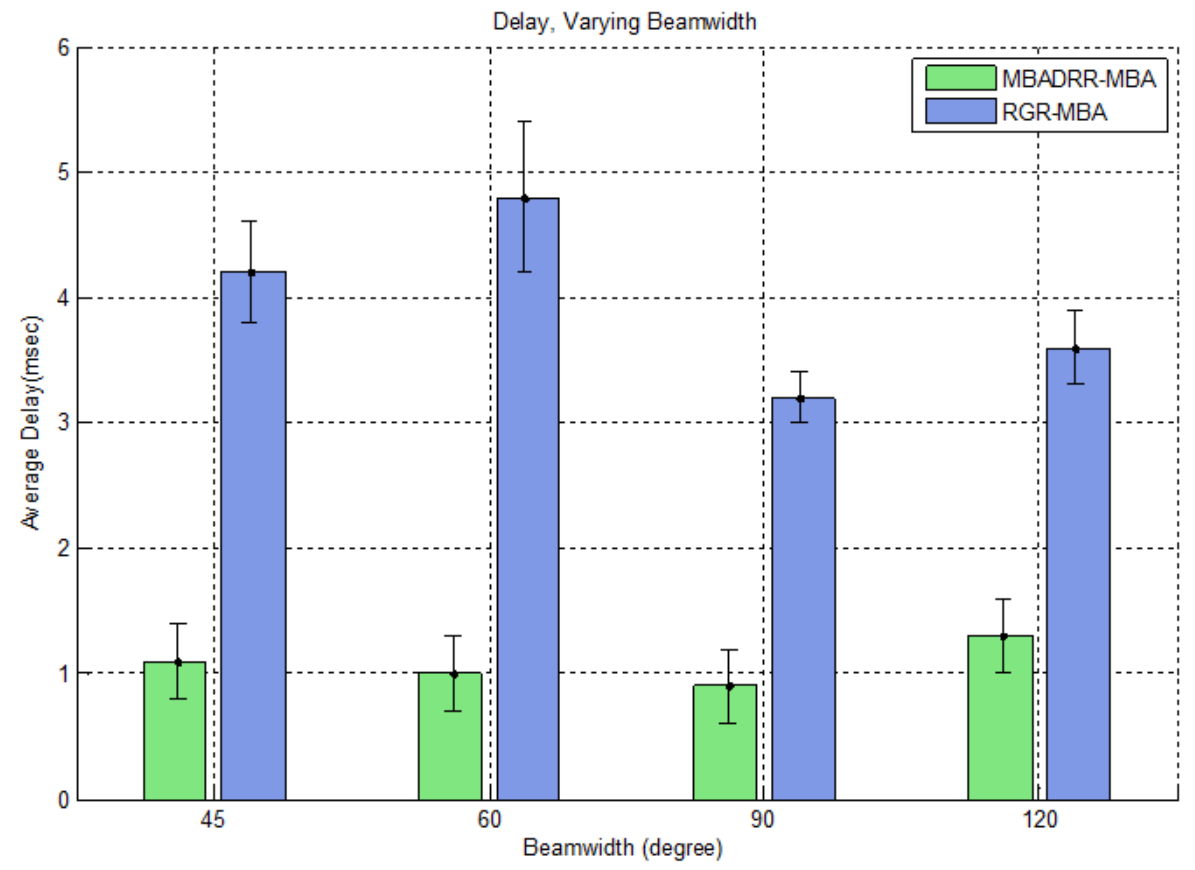

Figure 7.13: Delay, 10-Node Mobile Scenario, Varying Beamwidth

When we vary the beamwidth in a 25 -node 12 -flow network, we obtain the results in Figures 7.15 and 7.16. A clear trend now appears as expected: the delay increases as the beamwidth increases. With a larger beamwidth, we have less spatial reuse. Most importantly, more flows are serviced by the same beam of a given node, resulting 


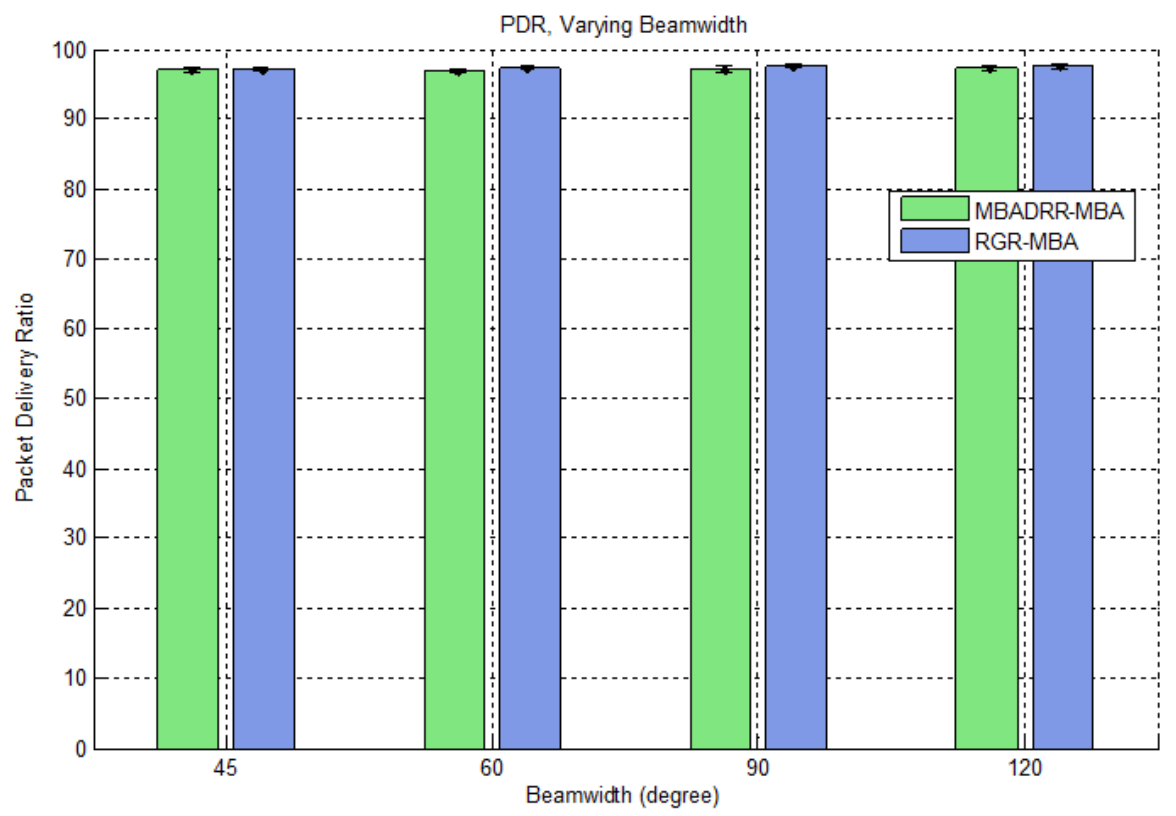

Figure 7.14: PDR, 10-Node Mobile Scenario, Varying Beamwidth

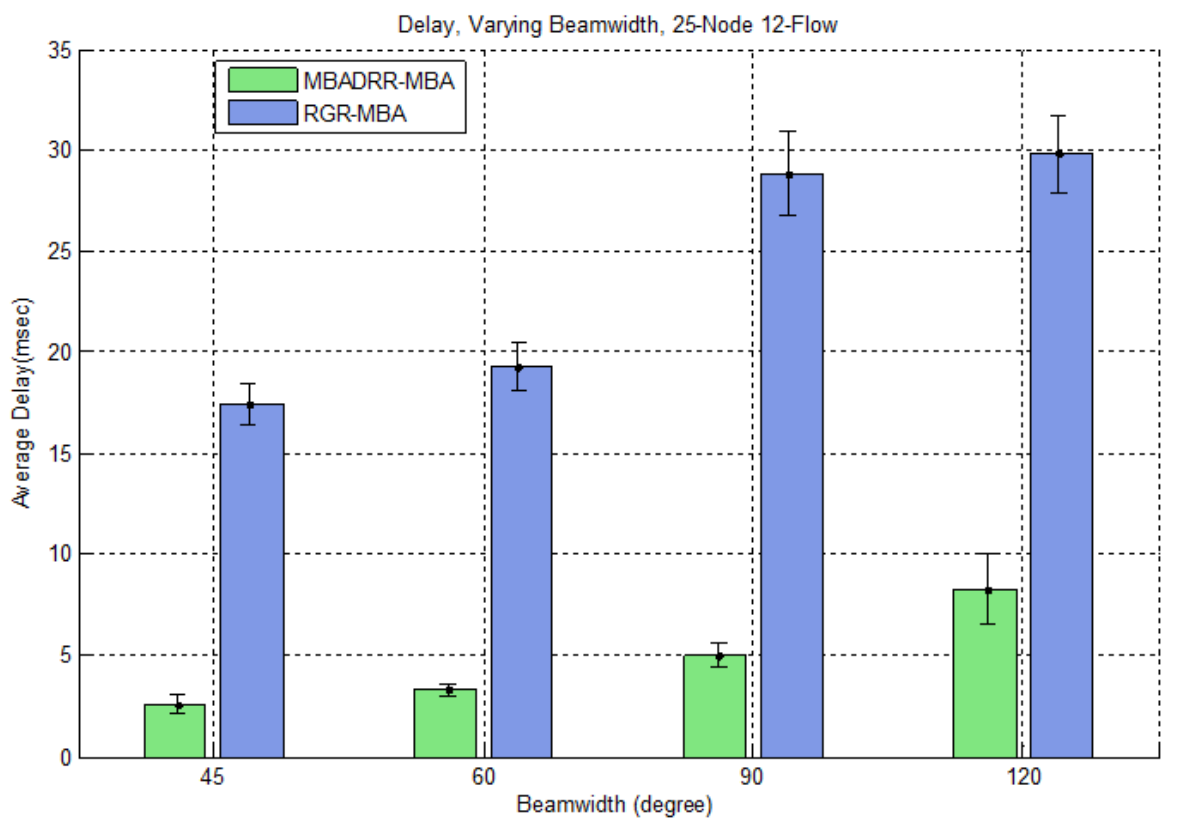

Figure 7.15: Delay, 25-Node Mobile Scenario, Varying Beamwidth

in more bridges being formed; hence the increase in E2E delay overall. 


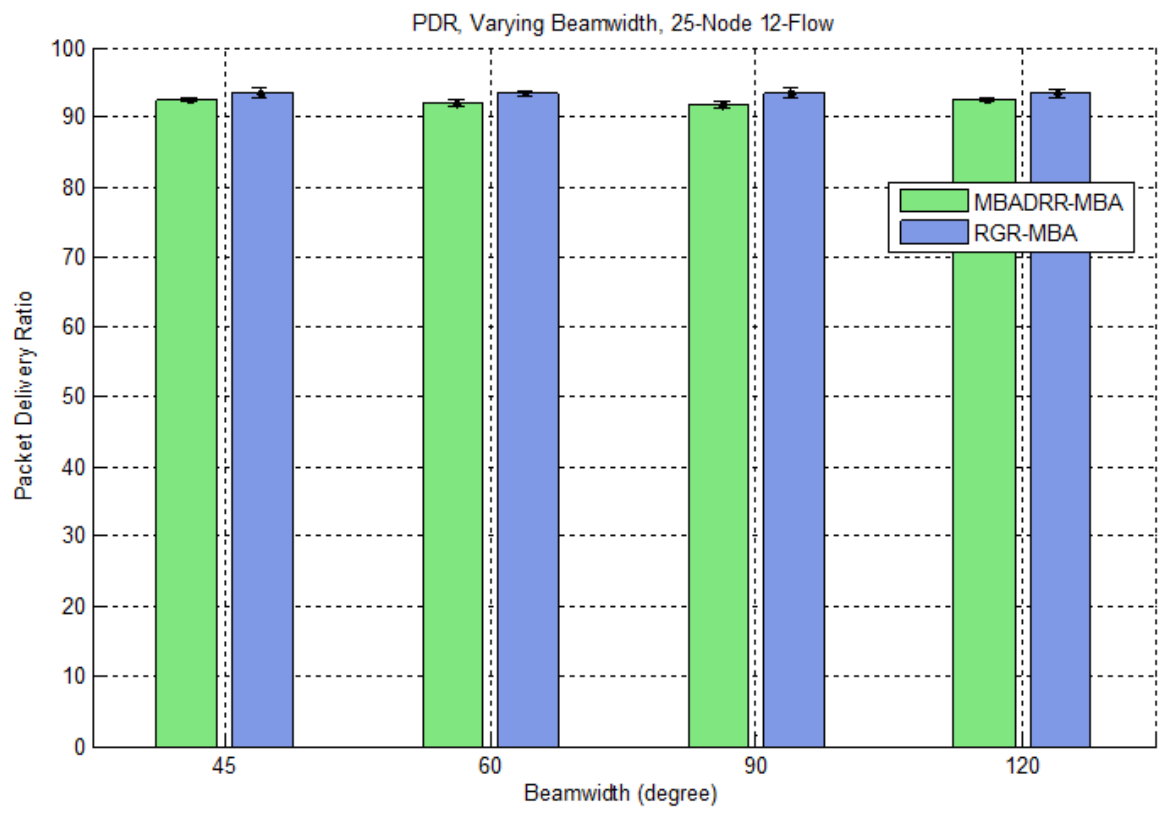

Figure 7.16: PDR, 25-Node Mobile Scenario, Varying Beamwidth

\subsection{Summary}

In this chapter, we have evaluated our MBA-DRR protocol in various scenarios in order to show that it achieves our goal of reducing the E2E delay in a multi-flow flying ad hoc network. The evaluation has been divided into three different parts. First, in a static scenario, we showed how the routes selected by MBA-DRR compare to the ones suggested by the optimization model, and to the ones selected by RGR, a shortest-route-based protocol. We showed how this reflected on the E2E delay reduction. Secondly, in a mobile scenario, we showed that MBA-DRR, running over a suitable multi-beam MAC, is advantageous over running RGR on all types of antennas (with their respective suitable MAC). Finally, we further compared MBA-DRR and RGR, both running over the same multi-beam MAC, when the network size grows, when the traffic intensity grows, and when the beamwidth of the MBA varies. All these comparisons/evaluations showed the superiority of our protocol in terms of reducing E2E delay in multi-flow static and mobile ad hoc networks.

Overall, we can now claim that we have been able to successfully reduce the E2E delay of a mobile multi-flow network of UAVs. Specifically, in a given scenario, we 
have been able to bring this delay from $700 \mathrm{~ms}$ down to just $9 \mathrm{~ms}$. This has been done in three major steps: a) a shift from omnidirectional antennas to single-beam directional antennas by means of selection of a new antenna type and the design (achieved in Chapter 2) of a MAC protocol to work with it; b) a shift from singlebeam directional antennas to multi-beam directional antennas by means of selection of a new antenna type and the design (achieved in Chapter 4) yet again of a MAC protocol to work with it; and finally c) a departure from the widespread shortest-route selection philosophy to a smarter route selection philosophy that exploits the benefits of multi-beam functionalities with the concepts of stars and bridges (introduced in Chapter 5) in the design a new routing protocol. 


\section{Chapter 8}

\section{Conclusions and Future Work}

\subsection{Conclusions}

The goal of this work was to design a routing protocol that reduces the E2E delay for delay-sensitive applications in multi-flow multi-hop ad hoc networks. The networks could be static or mobile. We have focused on delay reduction without sacrificing the PDR. The pursuit of this goal has been conducted in four major steps.

As a first step, we investigated the benefits of using SBAs in single-flow MANETs. We did so by comparing the performance of existing MANET routing protocols in SBA-MANETs. To be able to use SBAs, we designed a generic MAC protocol for

SBAs, the DbMAC protocol. Although several MBA MAC protocols are described in the literature, none is implemented in OPNET; hence our need to design and implement one. DbMAC works mostly like the standard IEEE 802.11b DCF MAC protocol but on a per-antenna-sector basis. Given this per-sector working, broadcast and unicast cannot operate the same as in the original IEEE 802.11b MAC protocol. To broadcast a frame, a node makes copies of that frame and send them, one at a time, using a different antenna sector for each copy. Broadcasting is done in either one or two rounds, depending on the availability of the medium. The investigation into the benefits of SBA-MANETs led to some interesting findings, one of which being that RGR was the best routing protocol to use as a starting point in designing an SBA-MANET-applicable protocol. However, we saw that latency was still an issue in our single-flow scenarios.

As a second step, we established that in order to go beyond the level of 
performance of RGR and reduce the E2E latency, we needed to explore the benefits of using MBAs. We reviewed the related literature and found that MBAs have been widely utilized in wireless (both infrastructure and infrastructureless) networks mostly to improve capacity and throughput. However, little work has been done to exploit the MPT/MPR potential of MBAs to minimize the E2E delay. Furthermore, we pointed out a popular methodology used in optimizing link scheduling proposals aimed at various performance improvements. The methodology consists of formulating a linear-programming problem for optimal solutions and then designing a heuristic protocol accordingly. Exploiting the benefits of MBAs is conditioned by having a suitable MBA MAC protocol. We only had descriptions of several MBA MAC protocols in the literature, but no actual MBA MAC protocol was available in OPNET. Therefore, we proposed the MBA-DbMAC protocol, a generic MAC protocol that implements basic functionalities of a MAC protocol and renders possible the basic operation of MBA-equipped nodes. We adopted a two-tier processing approach whereby the MAC layer is split into two artificial sub-layers: the controller sub-layer (materialized by one node-wide parent process) and the sector sub-layer (materialized by $N$ child processes, 1 child process for each of the $N$ sectors). This two-tier design approach is an elegant way of enforcing mode switches in the node while still leaving the full autonomy of medium access to sectors in their respective direction of competence. Other novel aspects of MBA-DbMAC are the decoupled-broadcasting or diversity-casting and the time window policy that we adopt in order to avoid critical chain transmission/reception.

In our third step, we defined a formal optimization model for delay reduction. We formulated determining the best route selection (best link scheduling) as an optimization problem that we solved using linear programming. As pointed out in the literature review, a popular methodology consists of optimizing link scheduling for various performance improvements, and then designing a heuristic protocol accordingly. In the analysis of the model, we introduced the concepts of star nodes and bridges. Our scenarios showed that the optimal link scheduling forms a lot of star nodes and avoids bridges unless there is a clear delay advantage in keeping them. Some bridges are necessary for the optimal result, as we end up getting enough benefit from the additional stars they allow to be created. The refinement of our optimization model with the introduction of beam considerations sustained the 
conclusions of the initial model.

In the last step, we designed and implemented MBA-DRR our delay-reducing routing protocol, based on the lessons learnt from the analysis of the optimization model. Though primarily based on AODV and RGR, there is a fundamental difference between MBA-DRR and those protocols in that MBA-DRR chooses routes not based on the shortest-path criteria, but rater on a more-stars-less-bridges preference basis. This preference on more-stars-less-bridges is dictated by analysis results of the optimization model that showed that: a) the shortest-path routes usually have a relatively high E2E delay in multi-flow scenarios, and b) when minimal E2E delay is attained, the routes feature a lot of star nodes and very few to none bridges. We defined two route selection metrics: SBR and PHC. These two metrics led to the SBR/PHC measure that helps compare different routes with the goal of choosing the one that lowers the E2E delay for all the flows. It is important to note that this delay reduction is not exclusive to MANETs. MBA-DRR equally reduces the E2E delay in static multi-flow MBA-based ad hoc networks. As a matter of fact, an evaluation on a multi-flow static scenario shows that MBA-DRR, with a delay of just $4.4 \mathrm{~ms}$, gets very close to the optimal solution that has a delay of $2.5 \mathrm{~ms}$. RGR comes far behind with a delay of $48 \mathrm{~ms}$. Finally, an evaluation on a multi-flow mobile scenario shows that, while the introduction of SBAs reduces the E2E delay from $700 \mathrm{~ms}$ to $40 \mathrm{~ms}$, and MBAs halve this to $20 \mathrm{~ms}$, MBA-DRR further cuts this delay to $9 \mathrm{~ms}$.

In summary, with the ultimate goal of employing MBAs to design a delay-reducing routing protocol for multi-flow ad hoc networks, we have ended up with a fourfold contribution. In effect, the four outputs of this work are: a MAC protocol for SBAs, a MAC protocol for MBAs, a delay-specific optimization model, and a delay-reducing routing protocol for multi-flow MANETs that also serves for static ad hoc networks.

\subsection{Future Work}

Going forward, there are a few avenues to pursue. One such avenues is to study how MBA-DRR fares with other traffic patterns. In this work, we have focused on one traffic pattern whereby the traffic flows are completely independent to one another. An interesting pattern to explore is one where the majority of the traffic goes to one 
or a few gateways.

At some point while evaluating MBA-DRR, we stumbled upon an intriguing related result: contrary to RGR, AODV is negatively affected by MBAs. In a future work/publication, we will conduct a thorough investigation to find out why this is the case.

On the MAC protocol front, it would be worthwhile to systematically compare our proposed MAC protocols (DbMAC and MBA-DbMAC) against some proposed alternatives in the literature. In that endeavor, we will need to implement the protocols in the literature ourselves based on their respective and rather limited descriptions, as the actual implementations are usually not readily available. That would add an extra layer of work before the actual comparison and analysis.

Furthermore, we fixed several parameters in the implementation of the MAC protocols. Varying and evaluating those parameters would be necessary to finding the best trade-offs. For example, we could analyze the impact of the length of the time window as a means to combat critical chain transmission and critical chain reception while ensuring concurrent packet transmission and concurrent packet reception.

In addition, given that, when using MBAs, not all the beams are expected to be used at every single transmission mode, gains in energy efficiency are expected. Further investigation (including measurements) into these energy savings with our proposed MBA-DbMAC protocol will be a next related step.

We have pointed out that having a delay optimization model allows to also meet other E2E delay-related objectives that might be dictated by the targeted applications. One such objective is keeping the individual E2E delay of all flows below a given bound; which speaks to QoS. In pursuing better QoS, we could expand our model to realize other objectives, including ones that are not delay-related.

Based on the lessons learned from the optimization model for E2E delay reduction, MBA-DRR pursued two behaviours: the promotion star nodes and the 
avoidance of bridges. It would be interesting to explore the possibility of simplifying the algorithm to focus on only one of these two behaviours; for example, have MBA-DRR only try to avoid bridges.

Another interesting avenue worth of exploration is to devise a solution that specifically targets the further improvement of the PDR in MANETs, i.e to get it as close as possible to $100 \%$. To that effect, we could employ multi-path routing and/or other strategies that directly make use of the availability of multiple beams per node.

To model mobility in this work, we have used the Random Waypoint model. However, as pointed out in [8], with RWP, a node is subject to sudden stops, sudden accelerations, and sudden speed changes. The same applies to direction, where a node can suddenly make a 180 degree turn. Airborne vehicles have certain mechanical and aerodynamic constraints that prevent them from making sharp turns. Despite the widespread usage of this mobility model, it is not very realistic. Therefore, re-evaluating our protocols under a more realistic mobility model would be welcome. Some of these more realistic mobility models include the Smooth-Turn model [110], the Gauss-Markov model [111], and our own Enhanced-Gauss-Markov model [106].

Finally, in this work, we have focused on 2D networks to prove a point (on E2E delay reduction). The natural next step will consist of expanding the work to 3D networks which are closer to real-life airborne networks. This will mostly imply using different simulation scenarios and settings. For example: we will redefine/expand our antenna model from 2D to 3D, the nodes' location that is shared in routing control packets will include a third coordinate (z), etc. Our protocols (MAC and routing), however, will stay the same. 


\section{List of References}

[1] K. Bao, F. Hu, E. Bentley, and S. Kumar, "Diamond-shaped mesh network routing with cross-layer design to explore the benefits of multi-beam smart antennas," in 2016 25th International Conference on Computer Communication and Networks (ICCCN), Aug 2016, pp. 1-5.

[2] V. Jain, A. Gupta, and D. P. Agrawal, "On-demand medium access in multihop wireless networks with multiple beam smart antennas," IEEE Transactions on Parallel and Distributed Systems, vol. 19, no. 4, pp. 489-502, April 2008.

[3] A. A. Abdullah, L. Cai, and F. Gebali, "DSDMAC: Dual sensing directional MAC protocol for ad hoc networks with directional antennas," IEEE Transactions on Vehicular Technology, vol. 61, no. 3, pp. 1266-1275, March 2012.

[4] A. Jayasuriya, S. Perreau, A. Dadej, and S. Gordon, "Hidden vs. exposed terminal problem in ad hoc networks," Proceedings of the Australian Telecommunication Networks and Applications Conference, 012004.

[5] X. Ji, H. Yu, G. Fan, and W. Fu, "SDGR: An SDN-based geographic routing protocol for VANET," in 2016 IEEE International Conference on Internet of Things (iThings) and IEEE Green Computing and Communications (GreenCom) and IEEE Cyber, Physical and Social Computing (CPSCom) and IEEE Smart Data (SmartData), Dec 2016, pp. 276-281.

[6] lker Bekmezci, O. K. Sahingoz, and amil Temel, "Flying ad-hoc networks (FANETs): A survey," Ad Hoc Networks, vol. 11, no. 3, pp. 1254 - 1270, 2013.

[7] Y. Zhang, X. Li, and M. G. Amin, "Mobile ad hoc networks exploiting multibeam antennas," in Handbook on Advancements in Smart Antenna Technologies for Wireless Networks. IGI Global, 2009, pp. 398-424.

[8] J.-D. Medjo Me Biomo, "Unmanned Aeronautical ad Hoc Networks: Enhancing the Reactive-Greedy-Reactive Protocol and Introducing a New Mobility Model," Master's thesis, Carleton University, Ottawa, ON, Canada, 2014.

[9] B. Shah and K.-I. Kim, "A survey on three-dimensional wireless ad hoc and sensor networks," International Journal of Distributed Sensor Networks, vol. 10, no. 7, p. 616014, 2014. [Online]. Available: https: //doi.org/10.1155/2014/616014 
[10] J.-D. Medjo Me Biomo, T. Kunz, and M. St-Hilaire, "Exploiting multiple beam antennas for end-to-end delay reduction in ad hoc networks," in 9th EAI International Conference on Ad Hoc Networks (AdHocNets 2017), September 2017.

[11] J.-D. Medjo Me Biomo, T. Kunz, and M. St-Hilaire, "Exploiting multi-beam antennas for end-to-end delay reduction in ad hoc networks," Mobile Networks and Applications, vol. 23, no. 5, pp. 1293-1305, Oct 2018.

[12] J.-D. Medjo Me Biomo, T. Kunz, and M. St-Hilaire, "Directional antennas in FANETs: A performance analysis of routing protocols," in 2017 International Conference on Selected Topics in Mobile and Wireless Networking (MoWNeT), May 2017, pp. 1-8.

[13] G. Boggia, P. Camarda, C. Cormio, and L. A. Grieco, "A BIBD-based MAC protocol for wireless ad hoc networks with directional antennas," in Communication Systems, Networks and Digital Signal Processing, 2008. CNSDSP 2008. 6th International Symposium on, July 2008, pp. 25-29.

[14] "OPNET: Optimized network engineering tool." [Online]. Available: https: //www.riverbed.com/products/steelcentral/opnet.html?redirect=opnet

[15] J.-D. Medjo Me Biomo, T. Kunz, and M. St-Hilaire, "Routing in unmanned aerial ad hoc networks: A recovery strategy for greedy geographic forwarding failure," in 2014 IEEE Wireless Communications and Networking Conference (WCNC), April 2014, pp. 2236-2241.

[16] — - "Routing in unmanned aerial ad hoc networks: Introducing a route reliability criterion," in 2014 7th IFIP Wireless and Mobile Networking Conference $(W M N C)$, May 2014, pp. 1-7.

[17] S. R. Das, C. E. Perkins, and E. M. Belding-Royer, "Ad hoc On-Demand Distance Vector (AODV) Routing," RFC 3561, Jul. 2003. [Online]. Available: https://tools.ietf.org/html/rfc3561

[18] T. J. Raval and J. S. Shah, "Network density based analysis of geographic routing protocol for random mobility of nodes in MANET," in 2011 International Conference on Emerging Trends in Networks and Computer Communications (ETNCC), April 2011, pp. 282-286.

[19] P. Jacquet, "Optimized Link State Routing Protocol (OLSR)," RFC 3626, Oct. 2003. [Online]. Available: https://rfc-editor.org/rfc/rfc3626.txt

[20] R. Shirani, M. St-Hilaire, T. Kunz, Y. Zhou, J. Li, and L. Lamont, "The performance of greedy geographic forwarding in unmanned aeronautical ad-hoc networks," in 2011 Ninth Annual Communication Networks and Services Research Conference, May 2011, pp. 161-166.

[21] A. Maghsoudlou, M. St-Hilaire, and T. Kunz, "A survey on geographic routing protocols for mobile ad hoc networks," Department of Systems and Computer Engineering, Carleton University, Ottawa, ON, Canada, Tech. Rep., 32011 , SCE-11-03. 
[22] Q. Zhao and H. Zhu, "An optimized AODV protocol in mobile ad hoc network," in 2008 4th International Conference on Wireless Communications, Networking and Mobile Computing, Oct 2008, pp. 1-4.

[23] T. Korakis, G. Jakllari, and L. Tassiulas, "CDR-MAC: A protocol for full exploitation of directional antennas in ad hoc wireless networks," IEEE Transactions on Mobile Computing, vol. 7, no. 2, pp. 145-155, Feb 2008.

[24] M. Takata, M. Bandai, and T. Watanabe, "A MAC protocol with directional antennas for deafness avoidance in ad hoc networks," in IEEE GLOBECOM 2007 - IEEE Global Telecommunications Conference, Nov 2007, pp. 620-625.

[25] S. Han, Y. C. Deng, and G. C. Gu, "A modified directional MAC protocol for using smart antenna in wireless ad hoc and sensor networks," in 2008 4th International Conference on Wireless Communications, Networking and Mobile Computing, Oct 2008, pp. 1-3.

[26] V. Pourgolzari and S. A. Ghorashi, "A CDMA-based MAC protocol for ad hoc networks with directional antennas," in Computer Networks and Distributed Systems (CNDS), 2011 International Symposium on, Feb 2011, pp. 73-77.

[27] J. J. Chang, W. Liao, and J. R. Lai, "On-reservation-based MAC protocol for IEEE 802.11 wireless ad hoc networks with directional antenna," IEEE Transactions on Vehicular Technology, vol. 60, no. 6, pp. 2669-2679, July 2011.

[28] M. Alenazi and C. Sahin, "Design improvement and implementation of 3d gaussmarkov mobility model," vol. 48, 012012.

[29] V. Tolety and V. Tolety, "Load reduction in ad hoc networks using mobile servers," 1999.

[30] P. Bahl, A. Adya, J. Padhye, and A. Walman, "Reconsidering wireless systems with multiple radios," SIGCOMM Comput. Commun. Rev., vol. 34, no. 5, pp. 39-46, Oct. 2004.

[31] R. Draves, J. Padhye, and B. Zill, "Routing in multi-radio, multi-hop wireless mesh networks," in Proceedings of the 10th Annual International Conference on Mobile Computing and Networking, ser. MobiCom '04. New York, NY, USA: ACM, 2004, pp. 114-128.

[32] J. H. Winters, "Smart antenna techniques and their application to wireless ad hoc networks," IEEE Wireless Communications, vol. 13, no. 4, pp. 77-83, Aug 2006 .

[33] A. Singh, P. Ramanathan, and B. V. Veen, "Spatial reuse through adaptive interference cancellation in multi-antenna wireless networks," in GLOBECOM '05. IEEE Global Telecommunications Conference, 2005., vol. 5, Dec 2005, pp. 3091-3096.

[34] Y. Zhang, X. Li, and M. G. Amin, "Multi-channel smart antennas in wireless networks," in 2006 Fortieth Asilomar Conference on Signals, Systems and Computers, Oct 2006, pp. 305-309. 
[35] X. Li, Y. Zhang, and M. G. Amin, "Performance evaluation of wireless networks exploiting multi-beam antennas in multipath environments," in 2007 International Waveform Diversity and Design Conference, June 2007, pp. 188-192.

[36] D. D. Perkins, H. D. Hughes, and C. B. Owen, "Factors affecting the performance of ad hoc networks," in 2002 IEEE International Conference on Communications. Conference Proceedings. ICC 2002 (Cat. No.02CH37333), vol. 4, April 2002, pp. 2048-2052 vol.4.

[37] C. S. R. Putta, K. B. Prasad, D. Ravilla, R. S. M. Nath, and M. L. R. Chandra, "Performance of ad hoc network routing protocols in IEEE 802.11," in 2010 International Conference on Computer and Communication Technology (ICCCT), Sep. 2010, pp. 371-376.

[38] S. Bandyopadhyay, S. Roy, and T. Ueda, Enhancing the Performance of Ad Hoc Wireless Networks with Smart Antennas. CRC Press, 2016.

[39] I. Martinez and J. Altuna, "A cross-layer design for ad hoc wireless networks with smart antennas and QoS support," in 2004 IEEE 15th International Symposium on Personal, Indoor and Mobile Radio Communications (IEEE Cat. No.04TH8754), vol. 1, Sept 2004, pp. 589-593 Vol.1.

[40] K. Chen, S. H. Shah, and K. Nahrstedt, "Cross-layer design for data accessibility in mobile ad hoc networks," vol. 21, no. 1, pp. 49-76, 2002.

[41] M. Zorzi, J. Zeidler, A. Anderson, B. Rao, J. Proakis, A. L. Swindlehurst, M. Jensen, and S. Krishnamurthy, "Cross-layer issues in MAC protocol design for MIMO ad hoc networks," IEEE Wireless Communications, vol. 13, no. 4, pp. 62-76, Aug 2006.

[42] M. F. Uddin, H. Alazemi, and C. Assi, "Joint routing, scheduling and variablewidth channel allocation for multi-hop WMNs," in 2010 IEEE International Conference on Communications, May 2010, pp. 1-6.

[43] L. Dong and A. P. Petropulu, "Cooperative wireless medium access with multibeam adaptive arrays," in IEEE 6th Workshop on Signal Processing Advances in Wireless Communications, 2005., June 2005, pp. 870-874.

[44] X. Li, Y. Zhang, and M. G. Amin, "Cooperative wireless medium access exploiting multi-beam adaptive arrays and relay selection," in 2008 4th IEEE International Conference on Circuits and Systems for Communications, May 2008, pp. 362-366.

[45] J. Wang, Y. Fang, and D. Wu, "Uplink medium access control for wlans with multi-beam access point," in GLOBECOM '05. IEEE Global Telecommunications Conference, 2005., vol. 5, Dec 2005, pp. 5 pp.-3016.

[46] — - "Enhancing the performance of medium access control for WLANs with multi-beam access point," IEEE Transactions on Wireless Communications, vol. 6, no. 2, pp. 556-565, Feb 2007. 
[47] Z. Tang, X. Xing, and F. Jiang, "Providing balanced and enhanced transmission for WLANs with multi-beam access point," in 6th Annual Communication Networks and Services Research Conference (cnsr 2008), May 2008, pp. 242248.

[48] G. Thomas, "Random access with multi-beam antenna arrays," in 2002 IEEE Wireless Communications and Networking Conference Record. WCNC 2002 (Cat. No.02TH8609), vol. 2, Mar 2002, pp. 749-756 vol.2.

[49] Z. T. Chou, C. Q. Huang, and J. M. Chang, "QoS provisioning for wireless LANs with multi-beam access point," IEEE Transactions on Mobile Computing, vol. 13, no. 9, pp. 2113-2127, Sept 2014.

[50] S. Say, N. Aomi, T. Ando, and S. Shimamoto, "Circularly multi-directional antenna arrays with spatial reuse based MAC for aerial sensor networks," in 2015 IEEE International Conference on Communication Workshop (ICCW), June 2015, pp. 2225-2230.

[51] G. Kuperman, R. Margolies, N. M. Jones, B. Proulx, and A. Narula-Tam, "Uncoordinated MAC for adaptive multi-beam directional networks: Analysis and evaluation," in 2016 25th International Conference on Computer Communication and Networks (ICCCN), Aug 2016, pp. 1-10.

[52] R. Verma, A. Prakash, P. K. Verma, N. Tyagi, and R. Tripathi, "A novel MAC protocol for MANETs using smart antenna system," in 2010 International Conference on Power, Control and Embedded Systems, Nov 2010, pp. 1-6.

[53] Y. X. Tian and K. J. Wu, "A novel dynamic TDMA protocol for ad hoc networks using directional antennas," in Electric Information and Control Engineering (ICEICE), 2011 International Conference on, April 2011, pp. 65-69.

[54] G. Wang, W. Li, and X. Cai, "MBDMAC: A MAC protocol for multi-beam directional antennas in wireless networks," in 2015 IEEE 12th International Conference on Mobile Ad Hoc and Sensor Systems, Oct 2015, pp. 486-487.

[55] G. Wang, P. Xiao, and W. Li, "A novel MAC protocol for wireless network using multi-beam directional antennas," in 2017 International Conference on Computing, Networking and Communications (ICNC), Jan 2017, pp. 36-40.

[56] F. Babich, M. Comisso, and A. Dorni, "A novel SIR-based access scheme for multi-packet communication in 802.11 networks," in 2012 IEEE International Conference on Communications (ICC), June 2012, pp. 4494-4498.

[57] F. Babich, M. Comisso, A. Crismani, and A. Dorni, "On the design of MAC protocols for multi-packet communication in IEEE 802.11 heterogeneous networks using adaptive antenna arrays," IEEE Transactions on Mobile Computing, vol. 14, no. 11, pp. 2332-2348, Nov 2015.

[58] A. Furtado, R. Oliveira, R. Dinis, and L. Bernardo, "A distributed MAC protocol for multi-packet reception wireless networks," in 2016 IEEE 27th Annual International Symposium on Personal, Indoor, and Mobile Radio Communications (PIMRC), Sept 2016, pp. 1-6. 
[59] J. Lin, C. Wu, S. Ohzahata, and T. Kato, "A QoS supporting ad hoc network protocol combing admission-based TDMA and 802.11 DCF," in The 16th AsiaPacific Network Operations and Management Symposium, Sept 2014, pp. 1-4.

[60] L. Bao and J. Garcia-Luna-Aceves, "Transmission scheduling in ad hoc networks with directional antennas," in Proceedings of the 8th Annual International Conference on Mobile Computing and Networking, ser. MobiCom '02. New York, NY, USA: ACM, 2002, pp. 48-58.

[61] W. Choi, D. Jung, H. N. Lee, and H. Lim, "Power control for multiple access communication systems with multi-packet reception capability," in 2009 IEEE 34th Conference on Local Computer Networks, Oct 2009, pp. 281-284.

[62] X. Wang and J. J. Garcia-Luna-Aceves, "Embracing interference in ad hoc networks using joint routing and scheduling with multiple packet reception," in IEEE INFOCOM 2008 - The 27th Conference on Computer Communications, April 2008.

[63] F. Babich and M. Comisso, "Analysis of asynchronous multi-packet reception in 802.11 distributed wireless networks," in GLOBECOM 2009 - 2009 IEEE Global Telecommunications Conference, Nov 2009, pp. 1-6.

[64] F. Babich, M. Comisso, A. Crismani, and A. Dorni, "Multi-packet communication in 802.11 networks by spatial reuse: From theory to protocol," in 2013 IEEE International Conference on Communications (ICC), June 2013, pp. 5105-5109.

[65] X. Li, Y. Zhang, and M. G. Amin, "Analytical throughput evaluation of wireless networks using multibeam antennas," in 2008 4th IEEE International Conference on Circuits and Systems for Communications, May 2008, pp. 397-401.

[66] B. Mumey, I. Judson, J. Tang, and Y. Xing, "Topology control in multihop wireless networks with multi-beam smart antennas," in 2012 International Conference on Computing, Networking and Communications (ICNC), Jan 2012, pp. 1020-1024.

[67] S. M. Rokonuzzaman, R. Pose, and I. Gondal, "A cross-layer approach for QoS topology control in wireless ad hoc networks," in TENCON 2009 - 2009 IEEE Region 10 Conference, Jan 2009, pp. 1-6.

[68] D. T. Bennett and T. X. Brown, "Optimal data scheduling of mobile clients serviced using beamforming antennas," in MILCOM 2012 - 2012 IEEE Military Communications Conference, Oct 2012, pp. 1-10.

[69] M. X. Cheng, X. Gong, Y. Xu, and L. Cai, "Link activity scheduling for minimum end-to-end latency in multihop wireless sensor networks," in 2011 IEEE Global Telecommunications Conference - GLOBECOM 2011, Dec 2011, pp. 1-5.

[70] J. Yackoski and C. Shen, "Method for scheduling transmissions in an ad hoc network," Jan. 3 2008, wO Patent App. PCT/US2007/014,769. 
[71] P. Thulasiraman and X. Shen, "Decoupled optimization of interference aware routing and scheduling for throughput maximization in wireless relay mesh networks," in 2009 6th IEEE Annual Communications Society Conference on Sensor, Mesh and Ad Hoc Communications and Networks Workshops, June 2009, pp. 1-6.

[72] Y. Zhou, Y. Fang, and M. Tanguay, "An optimized link scheduling technique for mobile ad hoc networks using directional antennas," in Military Communications Conference, MILCOM 2015 - 2015 IEEE, Oct 2015, pp. 714-719.

[73] D. J. Vergados, M. Koutsogiannaki, and D. D. Vergados, "Optimizing end-toend TDMA scheduling in ad-hoc networks on random topologies," in Proceedings of the 6th annual mediterranean ad hoc networking workshop (Med-Hoc-Net 2007), 2007, pp. 71-78.

[74] A. Ephremides and T. V. Truong, "Scheduling broadcasts in multihop radio networks," IEEE Transactions on communications, vol. 38, no. 4, pp. 456-460, 1990 .

[75] G. Wang and N. Ansari, "Optimal broadcast scheduling in packet radio networks using mean field annealing," IEEE Journal on selected areas in Communications, vol. 15, no. 2, pp. 250-260, 1997.

[76] H. Shi and L. Wang, "A hybrid neural network for optimal TDMA transmission scheduling in packet radio networks," in Neural Networks, 2005. IJCNN'05. Proceedings. 2005 IEEE International Joint Conference on, vol. 5. IEEE, 2005, pp. 3210-3213.

[77] J.-C. Chen, I. Ting, I. Chih-Lin, and J.-T. Chen, "A novel broadcast scheduling strategy using factor graphs and sum-product algorithm," in Global Telecommunications Conference, 2004. GLOBECOM'04. IEEE, vol. 6. IEEE, 2004, pp. $4048-4053$.

[78] S. Salcedo-Sanz, C. Bousoño-Calzón, and A. R. Figueiras-Vidal, "A mixed neural-genetic algorithm for the broadcast scheduling problem," IEEE Transactions on Wireless Communications, vol. 2, no. 2, pp. 277-283, 2003.

[79] D. D. Vergados, D. J. Vergados, C. Douligeris, and S. L. Tombros, "QoS-aware TDMA for end-to-end traffic scheduling in ad hoc networks," IEEE Wireless Communications, vol. 13, no. 5, 2006.

[80] D. J. Vergados, M. Koutsogiannaki, D. D. Vergados, V. Loumos, and N. Pantazis, "Enhanced end-to-end TDMA for wireless ad-hoc networks," in Computers and Communications, 2007. ISCC 2007. 12th IEEE Symposium on. IEEE, 2007, pp. MW-39.

[81] J. Tang, G. Xue, C. Chandler, and W. Zhang, "Link scheduling with power control for throughput enhancement in multihop wireless networks," IEEE Transactions on Vehicular Technology, vol. 55, no. 3, pp. 733-742, May 2006. 
[82] X. Meng, K. Tan, and Q. Zhang, "Joint routing and channel assignment in multi-radio wireless mesh networks," in 2006 IEEE International Conference on Communications, vol. 8, June 2006, pp. 3596-3601.

[83] K. Jain, J. Padhye, V. N. Padmanabhan, and L. Qiu, "Impact of interference on multi-hop wireless network performance," in Proceedings of the 9th Annual International Conference on Mobile Computing and Networking, ser. MobiCom '03. New York, NY, USA: ACM, 2003, pp. 66-80.

[84] P.-K. Huang, X. Lin, and C.-C. Wang, "A low-complexity congestion control and scheduling algorithm for multihop wireless networks with order-optimal perflow delay," IEEE/ACM Transactions on Networking (TON), vol. 21, no. 2, pp. 495-508, 2013.

[85] M. Kodialam and T. Nandagopal, "Characterizing achievable rates in multi-hop wireless networks: the joint routing and scheduling problem," in Proceedings of the 9th annual international conference on Mobile computing and networking. ACM, 2003, pp. 42-54.

[86] K. Hong, Y. Hua, and A. Swami, "Distributed and cooperative link scheduling for large-scale multihop wireless networks," EURASIP Journal on Wireless Communications and Networking, vol. 2007, p. 4, 2007.

[87] S. Dong, Q. Yang, F. Fu, and K. S. Kwak, "Distributed link scheduling for congestion control in multihop wireless network," in 2013 International Conference on Wireless Communications and Signal Processing, Oct 2013, pp. 1-5.

[88] K. Stamatiou, F. Rossetto, M. Haenggi, T. Javidi, J. R. Zeidler, and M. Zorzi, "A delay-minimizing routing strategy for wireless multi-hop networks," in Proceedings of the 7th International Conference on Modeling and Optimization in Mobile, Ad Hoc, and Wireless Networks, ser. WiOPT'09. Piscataway, NJ, USA: IEEE Press, 2009, pp. 500-505.

[89] M. Alvandi, M. Mehmet-Ali, and J. F. Hayes, "Delay optimization and crosslayer design in multihop wireless networks with network coding and successive interference cancelation," IEEE Journal on Selected Areas in Communications, vol. 33, no. 2, pp. 295-308, Feb 2015.

[90] G. Luo, S. Jia, Z. Liu, K. Zhu, and L. Zhang, "sdnMAC: A software defined networking based MAC protocol in VANETs," in 2016 IEEE/ACM 24th International Symposium on Quality of Service (IWQoS), June 2016, pp. 1-2.

[91] M. Abolhasan, J. Lipman, W. Ni, and B. Hagelstein, "Software-defined wireless networking: centralized, distributed, or hybrid?" IEEE Network, vol. 29, no. 4, pp. 32-38, July 2015.

[92] V. Kolar, S. Tilak, and N. B. Abu-Ghazaleh, "Avoiding head of line blocking in directional antenna MAC protocol," in 29th Annual IEEE International Conference on Local Computer Networks, Nov 2004, pp. 385-392. 
[93] A. P. Subramanian and S. R. Das, "Addressing deafness and hidden terminal problem in directional antenna based wireless multi-hop networks," Wireless Networks, vol. 16, no. 6, pp. 1557-1567, Aug 2010.

[94] V. Jain, A. Gupta, D. Lal, and D. P. Agrawal, "IEEE 802.11 DCF based MAC protocols for multiple beam antennas and their limitations," in IEEE International Conference on Mobile Adhoc and Sensor Systems Conference, 2005., Nov 2005, pp. 8 pp. -474.

[95] — _ "A cross layer MAC with explicit synchronization through intelligent feedback for multiple beam antennas," in GLOBECOM '05. IEEE Global Telecommunications Conference, 2005., vol. 6, Nov 2005, pp. 3196-3200.

[96] A. S. Acampora and S. I. A. Shah, "Multihop lightwave networks: a comparison of store-and-forward and hot-potato routing," IEEE Transactions on Communications, vol. 40, no. 6, pp. 1082-1090, June 1992.

[97] "Modeling with optimization programming language." [Online]. Available: https://www-01.ibm.com/software/commerce/optimization/modeling/

[98] "IBM ILOG CPLEX optimization studio." [Online]. Available: https: //www.ibm.com/jm-en/marketplace/ibm-ilog-cplex

[99] K. Liu, J. K. Y. Ng, V. C. S. Lee, S. H. Son, and I. Stojmenovic, "Cooperative data scheduling in hybrid vehicular ad hoc networks: VANET as a software defined network," IEEE/ACM Transactions on Networking, vol. 24, no. 3, pp. 1759-1773, June 2016.

[100] I. Ku, Y. Lu, M. Gerla, R. L. Gomes, F. Ongaro, and E. Cerqueira, "Towards software-defined VANET: Architecture and services," in 2014 13th Annual Mediterranean Ad Hoc Networking Workshop (MED-HOC-NET), June 2014, pp. 103-110.

[101] P. Baskett, Y. Shang, W. Zeng, and B. Guttersohn, "SDNAN: Software-defined networking in ad hoc networks of smartphones," in 2013 IEEE 10th Consumer Communications and Networking Conference (CCNC), Jan 2013, pp. 861-862.

[102] A. U. Khan and B. K. Ratha, "Time series prediction qos routing in software defined vehicular ad-hoc network," in 2015 International Conference on Man and Machine Interfacing (MAMI), Dec 2015, pp. 1-6.

[103] K. L. K. Sudheera, M. Ma, G. G. M. N. Ali, and P. H. J. Chong, "Delay efficient software defined networking based architecture for vehicular networks," in 2016 IEEE International Conference on Communication Systems (ICCS), Dec 2016, pp. 1-6.

[104] B. Dong, W. Wu, Z. Yang, and J. Li, "Software defined networking based ondemand routing protocol in vehicle ad hoc networks," in 2016 12th International Conference on Mobile Ad-Hoc and Sensor Networks (MSN), Dec 2016, pp. 207213. 
[105] T. Luo, H. P. Tan, and T. Q. S. Quek, "Sensor openflow: Enabling softwaredefined wireless sensor networks," IEEE Communications Letters, vol. 16, no. 11, pp. 1896-1899, November 2012.

[106] J.-D. Medjo Me Biomo, T. Kunz, and M. St-Hilaire, "An enhanced gaussmarkov mobility model for simulations of unmanned aerial ad hoc networks," in 2014 7th IFIP Wireless and Mobile Networking Conference (WMNC), May 2014, pp. 1-8.

[107] T. Kunz, J.-D. Medjo Me Biomo, and M. St-Hilaire, "NetAnalyzer: Analyzing dynamic network topologies," in 2015 8th IFIP Wireless and Mobile Networking Conference (WMNC), Oct 2015, pp. 64-71.

[108] J. Clapper, J. Young, J. Cartwright, and J. Grimes, "Unmanned systems roadmap 2007-2032," U.S Department of Defense, USA, Tech. Rep., 12 2007. [Online]. Available: http://www.globalsecurity.org/intell/library/ reports/2007/dod-unmanned-systems-roadmap_2007-2032.pdf

[109] L. Lin, Q. Sun, J. Li, and F. Yang, "A novel geographic position mobility oriented routing strategy for UAVs," Journal of Computational Information Systems, vol. 8, no. 2, pp. 709-716, Jan 2012.

[110] Y. Wan, K. Namuduri, Y. Zhou, and S. Fu, "A smooth-turn mobility model for airborne networks," IEEE Transactions on Vehicular Technology, vol. 62, no. 7, pp. 3359-3370, Sep. 2013.

[111] B. Liang and Z. J. Haas, "Predictive distance-based mobility management for pcs networks," in IEEE INFOCOM '99. Conference on Computer Communications. Proceedings. Eighteenth Annual Joint Conference of the IEEE Computer and Communications Societies. The Future is Now (Cat. No.99CH36320), vol. 3, March 1999, pp. 1377-1384 vol.3. 


\section{Appendix A}

\section{Additional Tables and Figures for Chapter 6}

\section{A.1 Routing Tables after Initial Route Discovery of All Flows.}

Table A.1: Routing Table of Node 1

\begin{tabular}{|c|c|c|c|c|c|c|c|c|}
\hline \multicolumn{10}{|c|}{ Routing Table of Node 1 } \\
\hline Dest & DestSeq\# & NextH & HopCnt & B2PH & B2NH & StarCnt & BridCnt & BridNumFlows \\
\hline 14 & 1 & 6 & 3 & - & - & 0 & 0 & 0 \\
\hline 2 & 1 & 2 & 1 & - & - & 0 & 0 & 0 \\
\hline 16 & 1 & 2 & 6 & - & - & 0 & 0 & 0 \\
\hline 3 & 1 & 2 & 2 & - & - & 0 & 0 & 0 \\
\hline 6 & -1 & 6 & 1 & - & - & 0 & 0 & 0 \\
\hline 15 & 0 & 2 & 5 & - & - & 0 & 0 & 0 \\
\hline
\end{tabular}

Table A.2: Routing Table of Node 2

\begin{tabular}{|c|c|c|c|c|c|c|c|c|}
\hline \multicolumn{10}{|c|}{ Routing Table of Node 2 } \\
\hline Dest & DestSeq\# & NextH & HopCnt & B2PH & B2NH & StarCnt & BridCnt & BridNumFlows \\
\hline 14 & 2 & 6 & 3 & - & - & 0 & 0 & 0 \\
\hline 16 & 1 & 6 & 5 & - & - & 0 & 0 & 0 \\
\hline 3 & 1 & 3 & 1 & - & - & 0 & 0 & 0 \\
\hline 6 & -1 & 6 & 1 & - & - & 0 & 0 & 0 \\
\hline 15 & 0 & 3 & 4 & - & - & 0 & 0 & 0 \\
\hline 1 & 1 & 1 & 1 & - & - & 0 & 0 & 0 \\
\hline
\end{tabular}


Table A.3: Routing Table of Node 3

\begin{tabular}{|c|c|c|c|c|c|c|c|c|}
\hline \multicolumn{10}{|c|}{ Routing Table of Node 3 } \\
\hline Dest & DestSeq\# & NextH & HopCnt & B2PH & B2NH & StarCnt & BridCnt & BridNumFlows \\
\hline 8 & -1 & 8 & 1 & - & - & 0 & 0 & 0 \\
\hline 4 & -1 & 4 & 1 & - & - & 0 & 0 & 0 \\
\hline 14 & 1 & 6 & 3 & - & - & 0 & 0 & 0 \\
\hline 2 & 1 & 2 & 1 & - & - & 0 & 0 & 0 \\
\hline 16 & 1 & 6 & 5 & - & - & 0 & 0 & 0 \\
\hline 13 & 2 & 6 & 3 & - & - & 0 & 0 & 0 \\
\hline 6 & -1 & 6 & 1 & - & - & 0 & 0 & 0 \\
\hline 15 & 0 & 8 & 3 & - & - & 0 & 0 & 0 \\
\hline 1 & 1 & 2 & 2 & - & - & 0 & 0 & 0 \\
\hline
\end{tabular}

Table A.4: Routing Table of Node 4

\begin{tabular}{|c|c|c|c|c|c|c|c|c|}
\hline \multicolumn{10}{|c|}{ Routing Table of Node 4 } \\
\hline Dest & DestSeq\# & NextH & HopCnt & B2PH & B2NH & StarCnt & BridCnt & BridNumFlows \\
\hline 8 & -1 & 8 & 1 & - & - & 0 & 0 & 0 \\
\hline 14 & 1 & 8 & 5 & - & - & 0 & 0 & 0 \\
\hline 2 & 2 & 3 & 2 & - & - & 0 & 0 & 0 \\
\hline 3 & -1 & 3 & 1 & - & - & 0 & 0 & 0 \\
\hline 13 & 1 & 8 & 6 & - & - & 0 & 0 & 0 \\
\hline 1 & 2 & 3 & 3 & - & - & 0 & 0 & 0 \\
\hline
\end{tabular}

Table A.5: Routing Table of Node 6

\begin{tabular}{|c|c|c|c|c|c|c|c|c|}
\hline \multicolumn{10}{|c|}{ Routing Table of Node 6} \\
\hline Dest & DestSeq\# & NextH & HopCnt & B2PH & B2NH & StarCnt & BridCnt & BridNumFlows \\
\hline 14 & 1 & 10 & 2 & - & - & 0 & 0 & 0 \\
\hline 2 & 1 & 2 & 1 & - & - & 0 & 0 & 0 \\
\hline 16 & 1 & 10 & 4 & - & - & 0 & 0 & 0 \\
\hline 3 & 1 & 3 & 1 & - & - & 0 & 0 & 0 \\
\hline 13 & 2 & 10 & 2 & - & - & 0 & 0 & 0 \\
\hline 10 & -1 & 10 & 1 & - & - & 0 & 0 & 0 \\
\hline 15 & 0 & 10 & 4 & - & - & 0 & 0 & 0 \\
\hline 1 & 1 & 1 & 1 & - & - & 0 & 0 & 0 \\
\hline
\end{tabular}


Table A.6: Routing Table of Node 8

\begin{tabular}{|c|c|c|c|c|c|c|c|c|}
\hline \multicolumn{10}{|c|}{ Routing Table of Node 8 } \\
\hline Dest & DestSeq\# & NextH & HopCnt & B2PH & B2NH & StarCnt & BridCnt & BridNumFlows \\
\hline 4 & -1 & 4 & 1 & - & - & 0 & 0 & 0 \\
\hline 14 & 1 & 12 & 4 & - & - & 0 & 0 & 0 \\
\hline 2 & 1 & 3 & 2 & - & - & 0 & 0 & 0 \\
\hline 12 & -1 & 12 & 1 & - & - & 0 & 0 & 0 \\
\hline 3 & 1 & 3 & 1 & - & - & 0 & 0 & 0 \\
\hline 13 & 1 & 12 & 5 & - & - & 0 & 0 & 0 \\
\hline 15 & 0 & 12 & 2 & - & - & 0 & 0 & 0 \\
\hline 1 & 1 & 3 & 3 & - & - & 0 & 0 & 0 \\
\hline
\end{tabular}

Table A.7: Routing Table of Node 10

\begin{tabular}{|c|c|c|c|c|c|c|c|c|}
\hline \multicolumn{10}{|c|}{ Routing Table of Node 10 } \\
\hline Dest & DestSeq\# & NextH & HopCnt & B2PH & B2NH & StarCnt & BridCnt & BridNumFlows \\
\hline 2 & 1 & 6 & 2 & - & - & 0 & 0 & 0 \\
\hline 14 & 1 & 14 & 1 & - & - & 0 & 0 & 0 \\
\hline 16 & 1 & 14 & 3 & - & - & 0 & 0 & 0 \\
\hline 3 & 1 & 6 & 2 & - & - & 0 & 0 & 0 \\
\hline 13 & 1 & 13 & 1 & - & - & 0 & 0 & 0 \\
\hline 6 & -1 & 6 & 1 & - & - & 0 & 0 & 0 \\
\hline 15 & -1 & 15 & 1 & - & - & 0 & 0 & 0 \\
\hline 1 & 1 & 6 & 2 & - & - & 0 & 0 & 0 \\
\hline
\end{tabular}

Table A.8: Routing Table of Node 12

\begin{tabular}{|c|c|c|c|c|c|c|c|c|}
\hline \multicolumn{10}{|c|}{ Routing Table of Node 12 } \\
\hline Dest & DestSeq\# & NextH & HopCnt & B2PH & B2NH & StarCnt & BridCnt & BridNumFlows \\
\hline 4 & 0 & 8 & 2 & - & - & 0 & 0 & 0 \\
\hline 8 & -1 & 8 & 1 & - & - & 0 & 0 & 0 \\
\hline 14 & 1 & 16 & 3 & - & - & 0 & 0 & 0 \\
\hline 2 & 2 & 8 & 3 & - & - & 0 & 0 & 0 \\
\hline 16 & 1 & 16 & 1 & - & - & 0 & 0 & 0 \\
\hline 3 & 2 & 8 & 2 & - & - & 0 & 0 & 0 \\
\hline 13 & 1 & 16 & 4 & - & - & 0 & 0 & 0 \\
\hline 15 & -1 & 15 & 1 & - & - & 0 & 0 & 0 \\
\hline 1 & 1 & 8 & 4 & - & - & 0 & 0 & 0 \\
\hline
\end{tabular}


Table A.9: Routing Table of Node 13

\begin{tabular}{|c|c|c|c|c|c|c|c|c|}
\hline \multicolumn{10}{|c|}{ Routing Table of Node 13 } \\
\hline Dest & DestSeq\# & NextH & HopCnt & B2PH & B2NH & StarCnt & BridCnt & BridNumFlows \\
\hline 4 & 0 & 14 & 6 & - & - & 0 & 0 & 0 \\
\hline 14 & 1 & 14 & 1 & - & - & 0 & 0 & 0 \\
\hline 16 & 1 & 14 & 3 & - & - & 0 & 0 & 0 \\
\hline 3 & 1 & 10 & 3 & - & - & 0 & 0 & 0 \\
\hline 10 & -1 & 10 & 1 & - & - & 0 & 0 & 0 \\
\hline 15 & 0 & 14 & 2 & - & - & 0 & 0 & 0 \\
\hline 1 & 1 & 10 & 3 & - & - & 0 & 0 & 0 \\
\hline
\end{tabular}

Table A.10: Routing Table of Node 14

\begin{tabular}{|c|c|c|c|c|c|c|c|c|}
\hline \multicolumn{10}{|c|}{ Routing Table of Node 14 } \\
\hline Dest & DestSeq\# & NextH & HopCnt & B2PH & B2NH & StarCnt & BridCnt & BridNumFlows \\
\hline 4 & 0 & 15 & 5 & - & - & 0 & 0 & 0 \\
\hline 2 & 1 & 10 & 3 & - & - & 0 & 0 & 0 \\
\hline 16 & 1 & 15 & 2 & - & - & 0 & 0 & 0 \\
\hline 3 & 2 & 10 & 3 & - & - & 0 & 0 & 0 \\
\hline 13 & 1 & 13 & 1 & - & - & 0 & 0 & 0 \\
\hline 10 & -1 & 10 & 1 & - & - & 0 & 0 & 0 \\
\hline 15 & -1 & 15 & 1 & - & - & 0 & 0 & 0 \\
\hline 1 & 1 & 13 & 4 & - & - & 0 & 0 & 0 \\
\hline
\end{tabular}

Table A.11: Routing Table of Node 15

\begin{tabular}{|c|c|c|c|c|c|c|c|c|}
\hline \multicolumn{10}{|c|}{ Routing Table of Node 15 } \\
\hline Dest & DestSeq\# & NextH & HopCnt & B2PH & B2NH & StarCnt & BridCnt & BridNumFlows \\
\hline 4 & 0 & 16 & 4 & - & - & 0 & 0 & 0 \\
\hline 2 & 1 & 10 & 3 & - & - & 0 & 0 & 0 \\
\hline 14 & 1 & 14 & 1 & - & - & 0 & 0 & 0 \\
\hline 12 & -1 & 12 & 1 & - & - & 0 & 0 & 0 \\
\hline 16 & 1 & 16 & 1 & - & - & 0 & 0 & 0 \\
\hline 3 & 1 & 10 & 3 & - & - & 0 & 0 & 0 \\
\hline 13 & 1 & 14 & 2 & - & - & 0 & 0 & 0 \\
\hline 10 & -1 & 10 & 1 & - & - & 0 & 0 & 0 \\
\hline 1 & 1 & 14 & 5 & - & - & 0 & 0 & 0 \\
\hline
\end{tabular}


Table A.12: Routing Table of Node 16

\begin{tabular}{|c|c|c|c|c|c|c|c|c|}
\hline \multicolumn{10}{|c|}{ Routing Table of Node 16 } \\
\hline Dest & DestSeq\# & NextH & HopCnt & B2PH & B2NH & StarCnt & BridCnt & BridNumFlows \\
\hline 4 & 0 & 12 & 3 & - & - & 0 & 0 & 0 \\
\hline 2 & 1 & 12 & 4 & - & - & 0 & 0 & 0 \\
\hline 14 & 1 & 15 & 2 & - & - & 0 & 0 & 0 \\
\hline 12 & -1 & 12 & 1 & - & - & 0 & 0 & 0 \\
\hline 3 & 1 & 15 & 4 & - & - & 0 & 0 & 0 \\
\hline 13 & 1 & 15 & 3 & - & - & 0 & 0 & 0 \\
\hline 15 & -1 & 15 & 1 & - & - & 0 & 0 & 0 \\
\hline 1 & 1 & 12 & 5 & - & - & 0 & 0 & 0 \\
\hline
\end{tabular}

Table A.13: Routing Table of Node 2

\begin{tabular}{|c|c|c|c|c|c|c|c|c|}
\hline \multicolumn{10}{|c|}{ Routing Table of Node 2 } \\
\hline Dest & DestSeq\# & NextH & HopCnt & B2PH & B2NH & StarCnt & BridCnt & BridNumFlows \\
\hline 14 & 2 & 6 & 3 & - & 2 & 0 & 0 & 0 \\
\hline 16 & 1 & 6 & 5 & - & - & 0 & 0 & 0 \\
\hline 3 & 1 & 3 & 1 & - & - & 0 & 0 & 0 \\
\hline 6 & -1 & 6 & 1 & - & - & 0 & 0 & 0 \\
\hline 15 & 0 & 3 & 4 & 3 & 1 & 0 & 0 & 0 \\
\hline 1 & 1 & 1 & 1 & 1 & 3 & 0 & 0 & 0 \\
\hline
\end{tabular}

Table A.14: Routing Table of Node 4

\begin{tabular}{|c|c|c|c|c|c|c|c|c|}
\hline \multicolumn{10}{|c|}{ Routing Table of Node 4 } \\
\hline Dest & DestSeq\# & NextH & HopCnt & B2PH & B2NH & StarCnt & BridCnt & BridNumFlows \\
\hline 8 & -1 & 8 & 1 & - & - & 0 & 0 & 0 \\
\hline 14 & 1 & 8 & 5 & - & - & 0 & 0 & 0 \\
\hline 2 & 2 & 3 & 2 & - & - & 0 & 0 & 0 \\
\hline 3 & -1 & 3 & 1 & - & - & 0 & 0 & 0 \\
\hline 13 & 1 & 8 & 6 & - & 2 & 0 & 0 & 0 \\
\hline 1 & 2 & 3 & 3 & - & - & 0 & 0 & 0 \\
\hline
\end{tabular}

\section{A.2 Routing Tables after Delivery of the 1st Data Packet of Each Flow.}


Table A.15: Routing Table of Node 8

\begin{tabular}{|c|c|c|c|c|c|c|c|c|}
\hline \multicolumn{10}{|c|}{ Routing Table of Node 8 } \\
\hline Dest & DestSeq\# & NextH & HopCnt & B2PH & B2NH & StarCnt & BridCnt & BridNumFlows \\
\hline 4 & -1 & 4 & 1 & 2 & 5 & 0 & 0 & 0 \\
\hline 14 & 1 & 12 & 4 & - & - & 0 & 0 & 0 \\
\hline 2 & 1 & 3 & 2 & - & - & 0 & 0 & 0 \\
\hline 12 & -1 & 12 & 1 & - & - & 0 & 0 & 0 \\
\hline 3 & 1 & 3 & 1 & - & - & 0 & 0 & 0 \\
\hline 13 & 1 & 12 & 5 & - & - & 0 & 0 & 0 \\
\hline 15 & 0 & 12 & 2 & 4 & 2 & 0 & 0 & 0 \\
\hline 1 & 1 & 3 & 3 & 2 & 4 & 0 & 0 & 0 \\
\hline
\end{tabular}

Table A.16: Routing Table of Node 10

\begin{tabular}{|c|c|c|c|c|c|c|c|c|}
\hline \multicolumn{10}{|c|}{ Routing Table of Node 10 } \\
\hline Dest & DestSeq\# & NextH & HopCnt & B2PH & B2NH & StarCnt & BridCnt & BridNumFlows \\
\hline 2 & 1 & 6 & 2 & - & - & 0 & 0 & 0 \\
\hline 14 & 1 & 14 & 1 & 5 & 2 & 0 & 0 & 0 \\
\hline 16 & 1 & 14 & 3 & - & - & 0 & 0 & 0 \\
\hline 3 & 1 & 6 & 2 & 2 & 5 & 0 & 0 & 0 \\
\hline 13 & 1 & 13 & 1 & 5 & 3 & 0 & 0 & 0 \\
\hline 6 & -1 & 6 & 1 & - & - & 0 & 0 & 0 \\
\hline 15 & -1 & 15 & 1 & - & - & 0 & 0 & 0 \\
\hline 1 & 1 & 6 & 2 & - & - & 0 & 0 & 0 \\
\hline
\end{tabular}

Table A.17: Routing Table of Node 12

\begin{tabular}{|c|c|c|c|c|c|c|c|c|}
\hline \multicolumn{10}{|c|}{ Routing Table of Node 12 } \\
\hline Dest & DestSeq\# & NextH & HopCnt & B2PH & B2NH & StarCnt & BridCnt & BridNumFlows \\
\hline 4 & 0 & 8 & 2 & 2 & 5 & 0 & 0 & 0 \\
\hline 8 & -1 & 8 & 1 & - & - & 0 & 0 & 0 \\
\hline 14 & 1 & 16 & 3 & - & - & 0 & 0 & 0 \\
\hline 2 & 2 & 8 & 3 & - & - & 0 & 0 & 0 \\
\hline 16 & 1 & 16 & 1 & - & - & 0 & 0 & 0 \\
\hline 3 & 2 & 8 & 2 & - & - & 0 & 0 & 0 \\
\hline 13 & 1 & 16 & 4 & - & - & 0 & 0 & 0 \\
\hline 15 & -1 & 15 & 1 & 5 & 3 & 0 & 0 & 0 \\
\hline 1 & 1 & 8 & 4 & 2 & 5 & 0 & 0 & 0 \\
\hline
\end{tabular}


Table A.18: Routing Table of Node 13

\begin{tabular}{|c|c|c|c|c|c|c|c|c|}
\hline \multicolumn{10}{|c|}{ Routing Table of Node 13 } \\
\hline Dest & DestSeq\# & NextH & HopCnt & B2PH & B2NH & StarCnt & BridCnt & BridNumFlows \\
\hline 4 & 0 & 14 & 6 & - & 1 & 0 & 0 & 0 \\
\hline 14 & 1 & 14 & 1 & - & - & 0 & 0 & 0 \\
\hline 16 & 1 & 14 & 3 & - & - & 0 & 0 & 0 \\
\hline 3 & 1 & 10 & 3 & - & 6 & 0 & 0 & 0 \\
\hline 10 & -1 & 10 & 1 & - & - & 0 & 0 & 0 \\
\hline 15 & 0 & 14 & 2 & - & - & 0 & 0 & 0 \\
\hline 1 & 1 & 10 & 3 & - & - & 0 & 0 & 0 \\
\hline
\end{tabular}

Table A.19: Routing Table of Node 14

\begin{tabular}{|c|c|c|c|c|c|c|c|c|}
\hline \multicolumn{10}{|c|}{ Routing Table of Node 14 } \\
\hline Dest & DestSeq\# & NextH & HopCnt & B2PH & B2NH & StarCnt & BridCnt & BridNumFlows \\
\hline 4 & 0 & 15 & 5 & 4 & 6 & 0 & 0 & 0 \\
\hline 2 & 1 & 10 & 3 & - & 5 & 0 & 0 & 0 \\
\hline 16 & 1 & 15 & 2 & - & - & 0 & 0 & 0 \\
\hline 3 & 2 & 10 & 3 & - & 5 & 0 & 0 & 0 \\
\hline 13 & 1 & 13 & 1 & - & - & 0 & 0 & 0 \\
\hline 10 & -1 & 10 & 1 & - & - & 0 & 0 & 0 \\
\hline 15 & -1 & 15 & 1 & - & - & 0 & 0 & 0 \\
\hline 1 & 1 & 13 & 4 & - & - & 0 & 0 & 0 \\
\hline
\end{tabular}

Table A.20: Routing Table of Node 15

\begin{tabular}{|c|c|c|c|c|c|c|c|c|}
\hline \multicolumn{10}{|c|}{ Routing Table of Node 15 } \\
\hline Dest & DestSeq\# & NextH & HopCnt & B2PH & B2NH & StarCnt & BridCnt & BridNumFlows \\
\hline 4 & 0 & 16 & 4 & 3 & 1 & 0 & 0 & 0 \\
\hline 2 & 1 & 10 & 3 & - & - & 0 & 0 & 0 \\
\hline 14 & 1 & 14 & 1 & - & - & 0 & 0 & 0 \\
\hline 12 & -1 & 12 & 1 & - & - & 0 & 0 & 0 \\
\hline 16 & 1 & 16 & 1 & - & - & 0 & 0 & 0 \\
\hline 3 & 1 & 10 & 3 & - & - & 0 & 0 & 0 \\
\hline 13 & 1 & 14 & 2 & - & - & 0 & 0 & 0 \\
\hline 10 & -1 & 10 & 1 & - & - & 0 & 0 & 0 \\
\hline 1 & 1 & 14 & 5 & - & 6 & 0 & 0 & 0 \\
\hline
\end{tabular}


Table A.21: Routing Table of Node 16

\begin{tabular}{|c|c|c|c|c|c|c|c|c|}
\hline \multicolumn{10}{|c|}{ Routing Table of Node 16 } \\
\hline Dest & DestSeq\# & NextH & HopCnt & B2PH & B2NH & StarCnt & BridCnt & BridNumFlows \\
\hline 4 & 0 & 12 & 3 & 4 & 5 & 0 & 0 & 0 \\
\hline 2 & 1 & 12 & 4 & - & - & 0 & 0 & 0 \\
\hline 14 & 1 & 15 & 2 & - & - & 0 & 0 & 0 \\
\hline 12 & -1 & 12 & 1 & - & - & 0 & 0 & 0 \\
\hline 3 & 1 & 15 & 4 & - & - & 0 & 0 & 0 \\
\hline 13 & 1 & 15 & 3 & - & - & 0 & 0 & 0 \\
\hline 15 & -1 & 15 & 1 & - & - & 0 & 0 & 0 \\
\hline 1 & 1 & 12 & 5 & - & 5 & 0 & 0 & 0 \\
\hline
\end{tabular}


Appendix B

Additional Tables and Figures for Chapter 7 


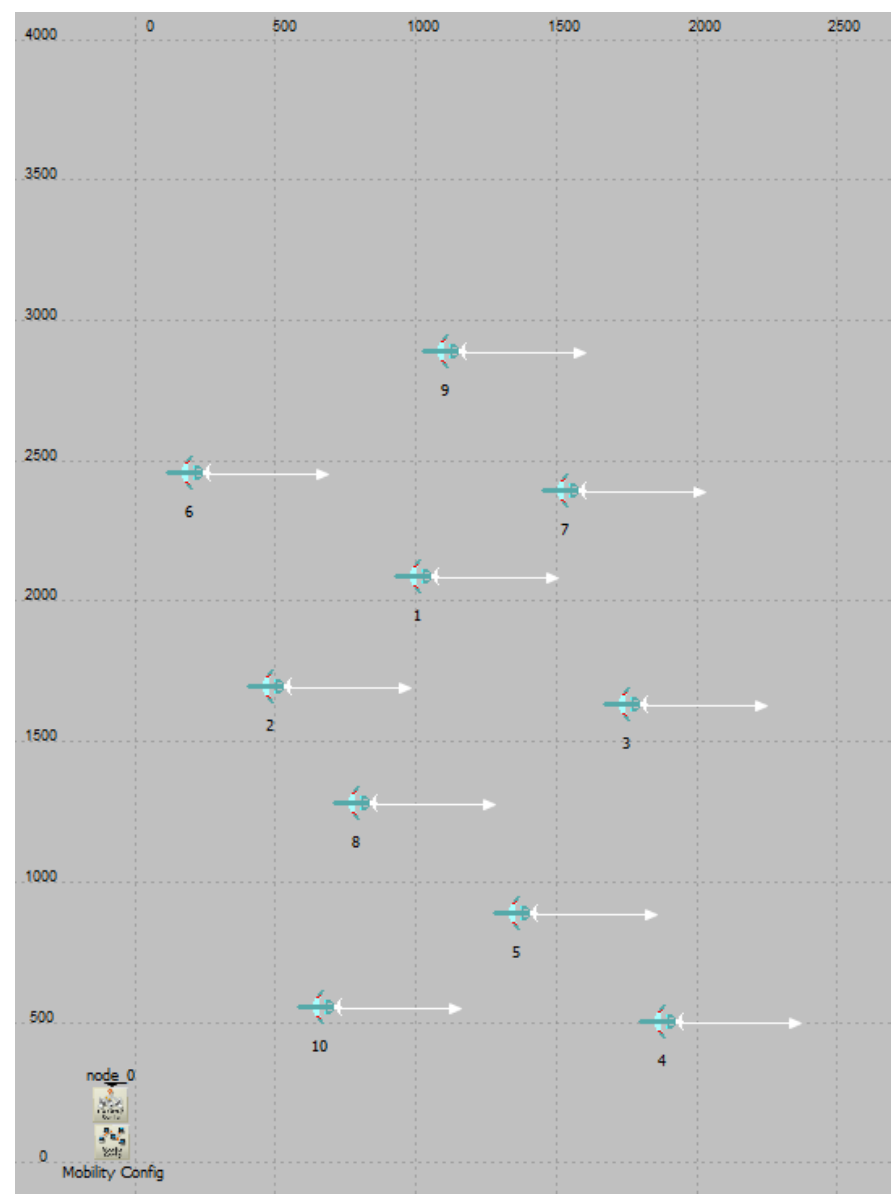

Figure B.1: Random 10-Node 5-Flow Static Scenario 1

Table B.1: Optimal Link Scheduling for 10-Node Scenario 1

\begin{tabular}{lccccc}
\hline Traffic flow & slot 1 & slot 2 & slot 3 & slot 4 & slot 5 \\
\hline \hline $9 \longrightarrow 10$ & $(9,1)$ & $(1,8)$ & $(8,10)$ & & \\
$4 \longrightarrow 6$ & $(4,5)$ & $(5,8)$ & $(8,2)$ & $(2,6)$ & \\
$5 \longrightarrow 7$ & & $(5,3)$ & $(3,7)$ & & \\
$1 \longrightarrow 4$ & & $(1,3)$ & $(3,5)$ & $(5,4)$ & \\
$6 \longrightarrow 5$ & $(6,2)$ & $(2,8)$ & $(8,5)$ & & \\
\hline
\end{tabular}




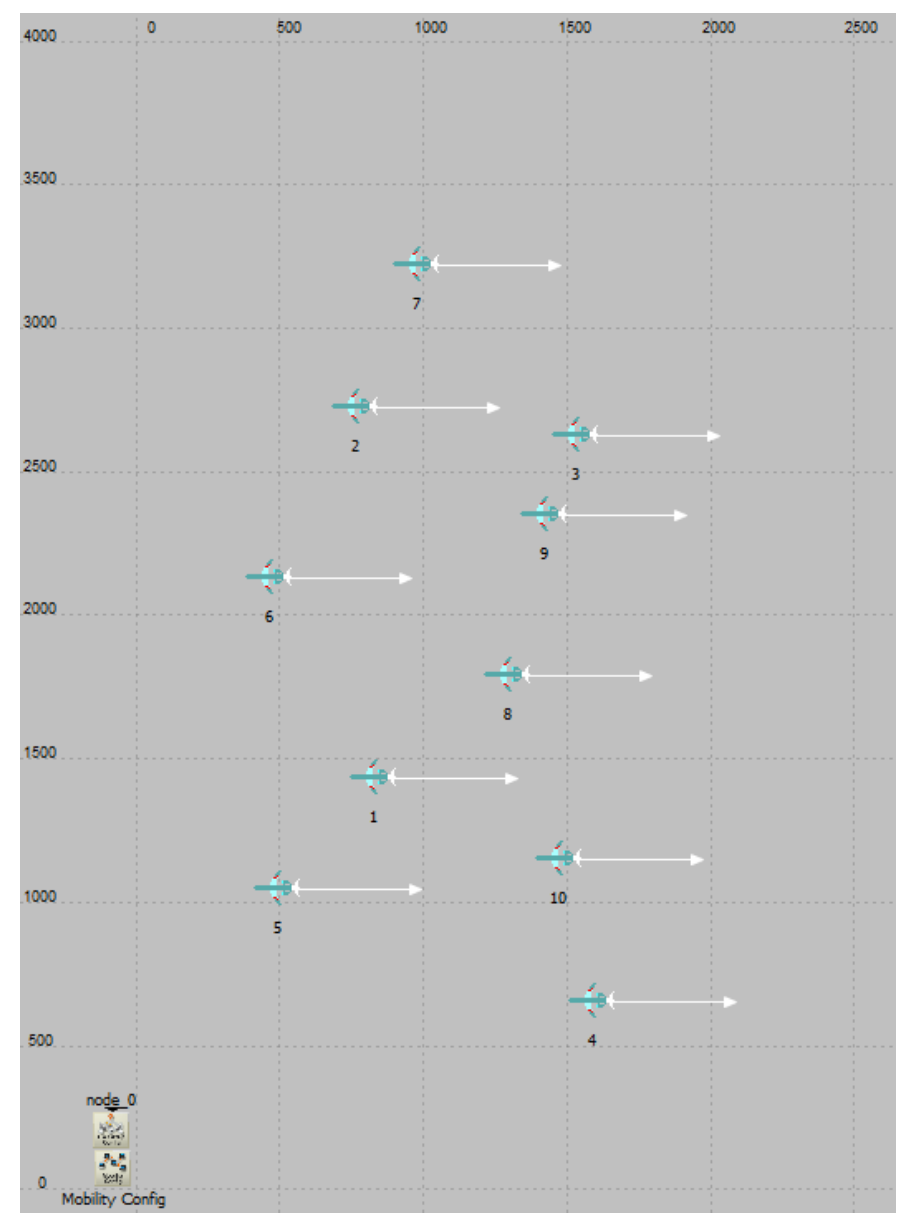

Figure B.2: Random 10-Node 5-Flow Static Scenario 2

Table B.2: Optimal Link Scheduling for 10-Node Scenario 2

\begin{tabular}{lccccc}
\hline Traffic flow & slot 1 & slot 2 & slot 3 & slot 4 & slot 5 \\
\hline \hline $4 \longrightarrow 7$ & & $(4,5)$ & $(5,8)$ & $(8,1)$ & $(1,7)$ \\
$10 \longrightarrow 2$ & $(10,8)$ & $(8,2)$ & & & \\
$5 \longrightarrow 3$ & $(5,3)$ & & & & \\
$1 \longrightarrow 2$ & $(1,2)$ & & & & \\
$7 \longrightarrow 10$ & $(7,3)$ & $(3,5)$ & $(5,10)$ & & \\
\hline
\end{tabular}




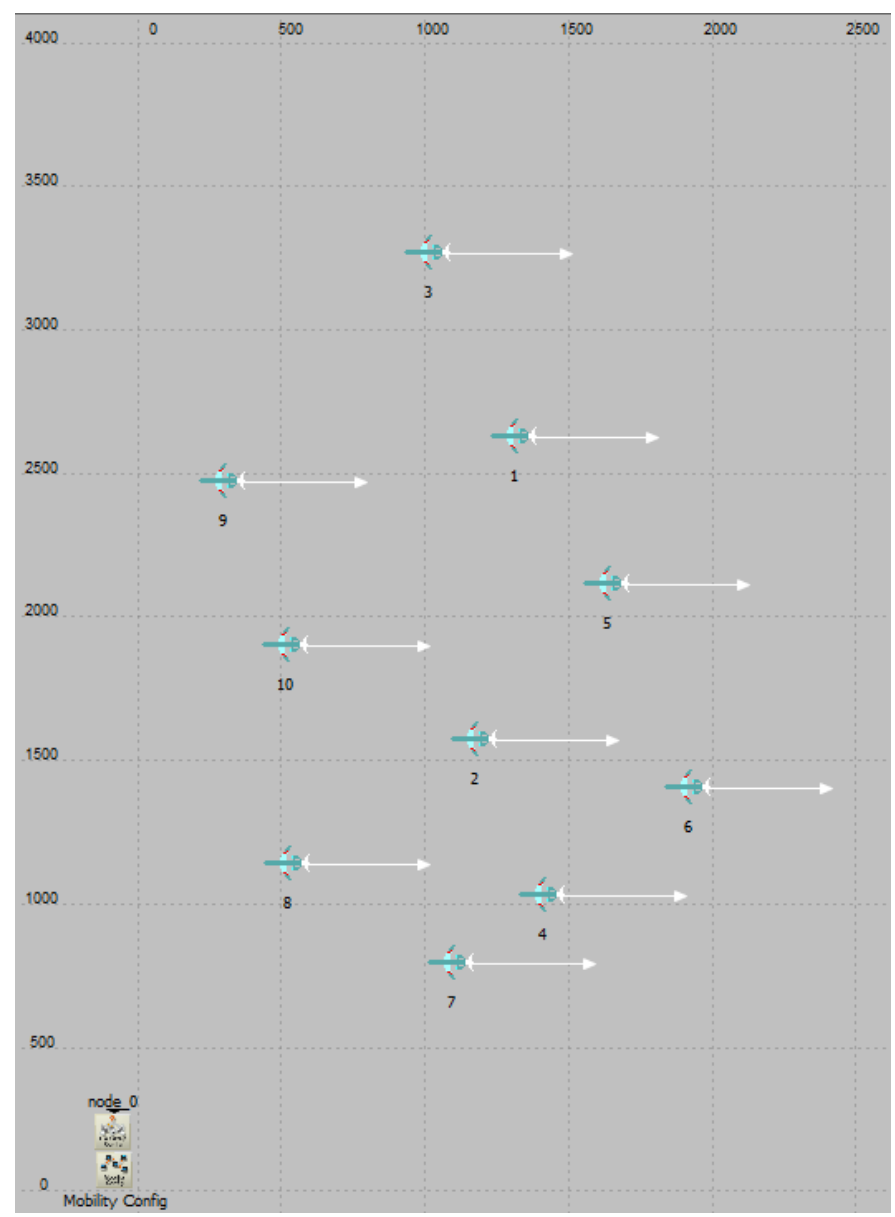

Figure B.3: Random 10-Node 5-Flow Static Scenario 3

Table B.3: Optimal Link Scheduling for 10-Node Scenario 3

\begin{tabular}{llllll}
\hline Traffic flow & slot 1 & slot 2 & slot 3 & slot 4 & slot 5 \\
\hline \hline $3 \longrightarrow 7$ & $(3,7)$ & & & & \\
$8 \longrightarrow 1$ & $(8,1)$ & & & & \\
$9 \longrightarrow 6$ & $(9,1)$ & $(1,6)$ & & & \\
$7 \longrightarrow 5$ & & $(7,3)$ & $(3,5)$ & & \\
$4 \longrightarrow 9$ & $(4,5)$ & $(5,3)$ & $(3,1)$ & $(1,9)$ & \\
\hline
\end{tabular}




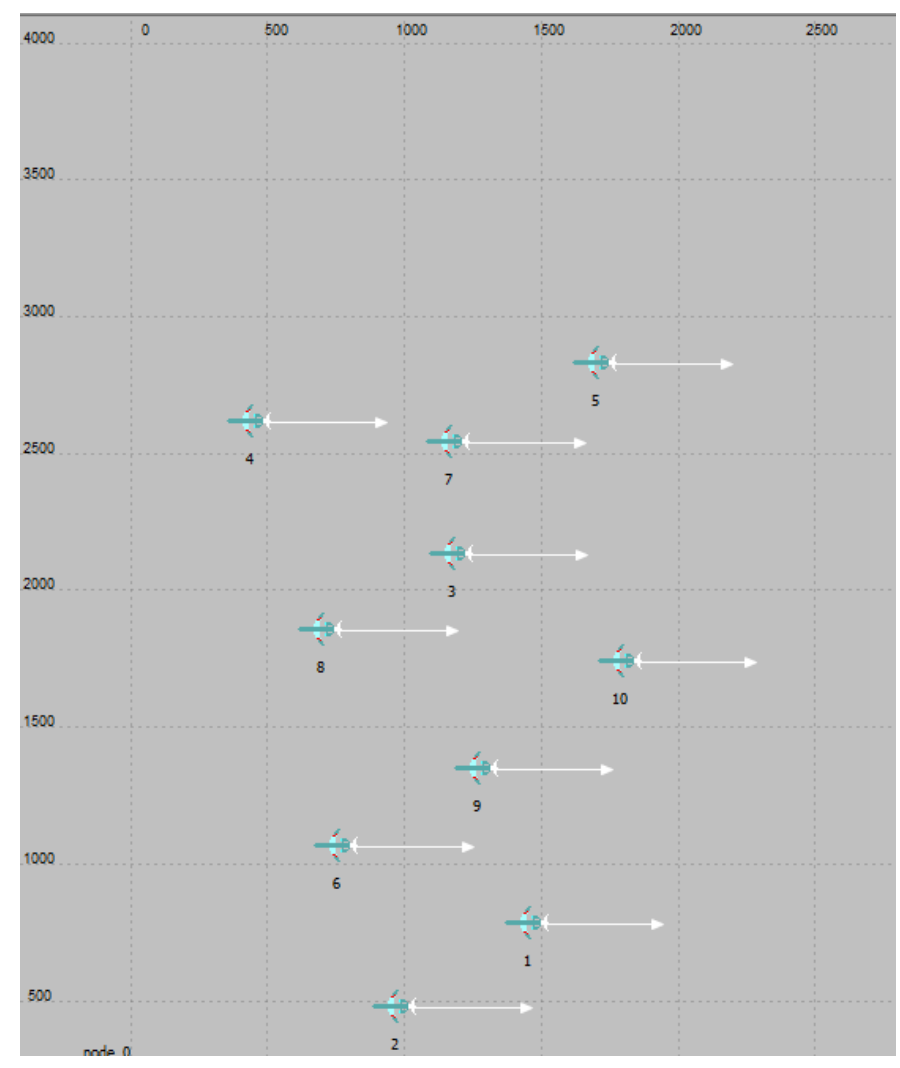

Figure B.4: Random 10-Node 5-Flow Static Scenario 4

Table B.4: Optimal Link Scheduling for 10-Node Scenario 4

\begin{tabular}{lccccc}
\hline Traffic flow & slot 1 & slot 2 & slot 3 & slot 4 & slot 5 \\
\hline \hline $2 \longrightarrow 5$ & $(2,8)$ & $(8,5)$ & & & \\
$1 \longrightarrow 4$ & $(1,3)$ & $(3,5)$ & $(5,4)$ & & \\
$7 \longrightarrow 6$ & & $(7,1)$ & $(1,6)$ & & \\
$4 \longrightarrow 10$ & & $(4,5)$ & $(5,10)$ & & \\
$5 \longrightarrow 9$ & $(5,3)$ & $(3,1)$ & $(1,9)$ & & \\
\hline
\end{tabular}




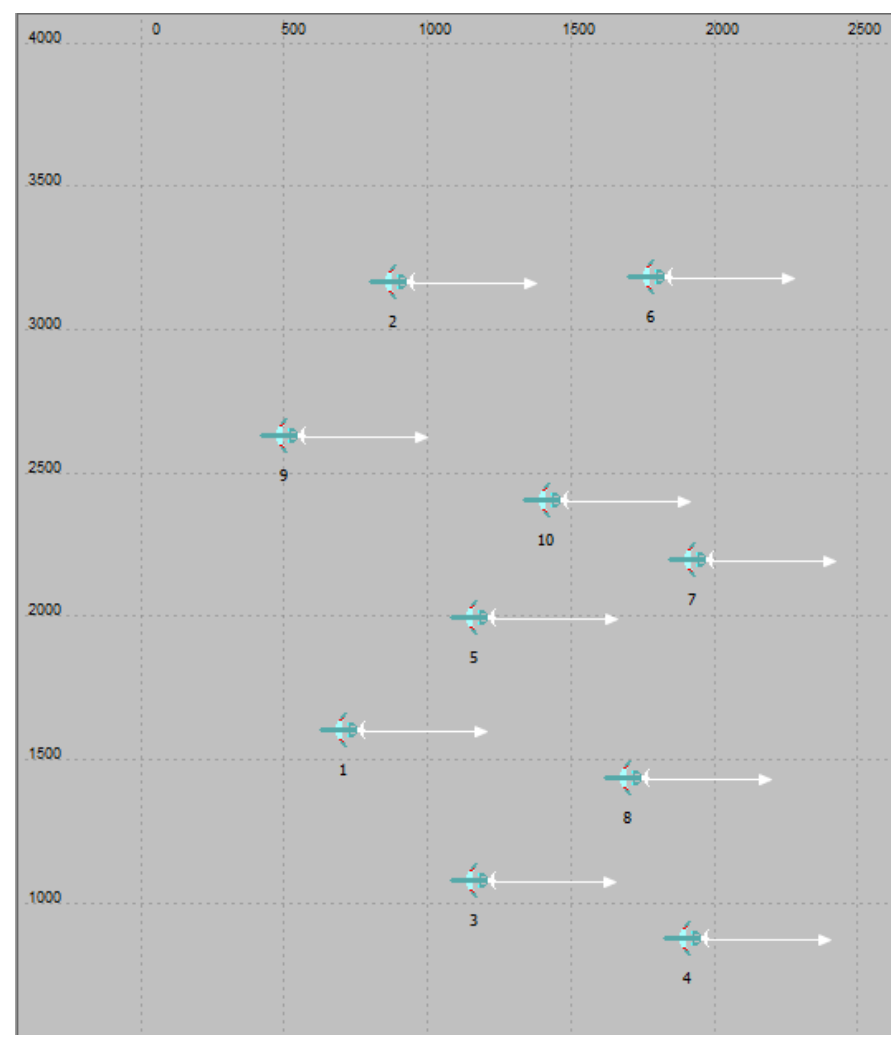

Figure B.5: Random 10-Node 5-Flow Static Scenario 5

Table B.5: Optimal Link Scheduling for 10-Node Scenario 5

\begin{tabular}{lccccc}
\hline Traffic flow & slot 1 & slot 2 & slot 3 & slot 4 & slot 5 \\
\hline \hline $2 \longrightarrow 4$ & $(2,8)$ & & $(8,5)$ & $(5,4)$ & \\
$3 \longrightarrow 6$ & $(3,1)$ & $(1,6)$ & & & \\
$9 \longrightarrow 8$ & $(9,1)$ & $(1,8)$ & & & \\
$6 \longrightarrow 1$ & $(6,1)$ & & & & \\
$4 \longrightarrow 10$ & $(4,5)$ & $(5,10)$ & & & \\
\hline
\end{tabular}




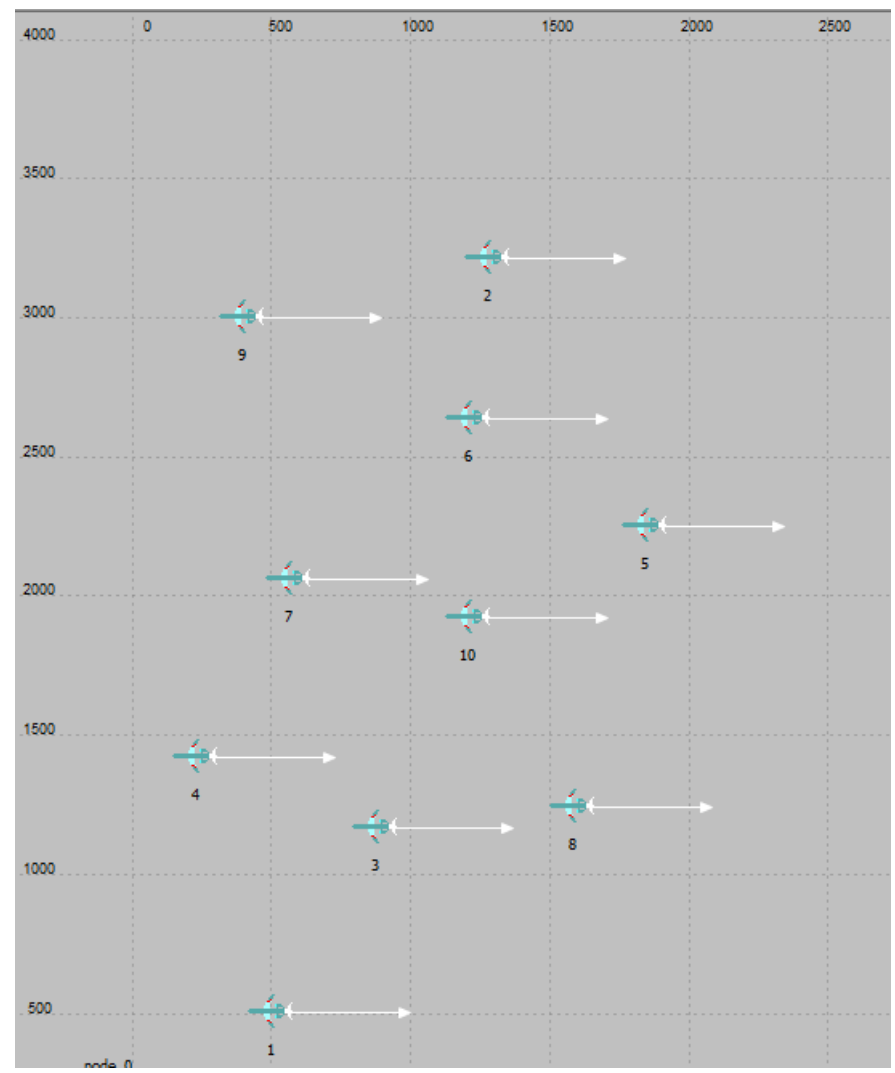

Figure B.6: Random 10-Node 5-Flow Static Scenario 6

Table B.6: Optimal Link Scheduling for 10-Node Scenario 6

\begin{tabular}{lccccc}
\hline Traffic flow & slot 1 & slot 2 & slot 3 & slot 4 & slot 5 \\
\hline \hline $1 \longrightarrow 2$ & $(1,2)$ & & & & \\
$3 \longrightarrow 9$ & $(3,7)$ & $(7,9)$ & & & \\
$4 \longrightarrow 6$ & $(4,5)$ & $(5,3)$ & $(3,1)$ & $(1,6)$ & \\
$5 \longrightarrow 1$ & & $(5,8)$ & $(8,1)$ & & \\
$9 \longrightarrow 8$ & $(9,7)$ & $(7,3)$ & $(3,5)$ & $(5,8)$ & \\
\hline
\end{tabular}




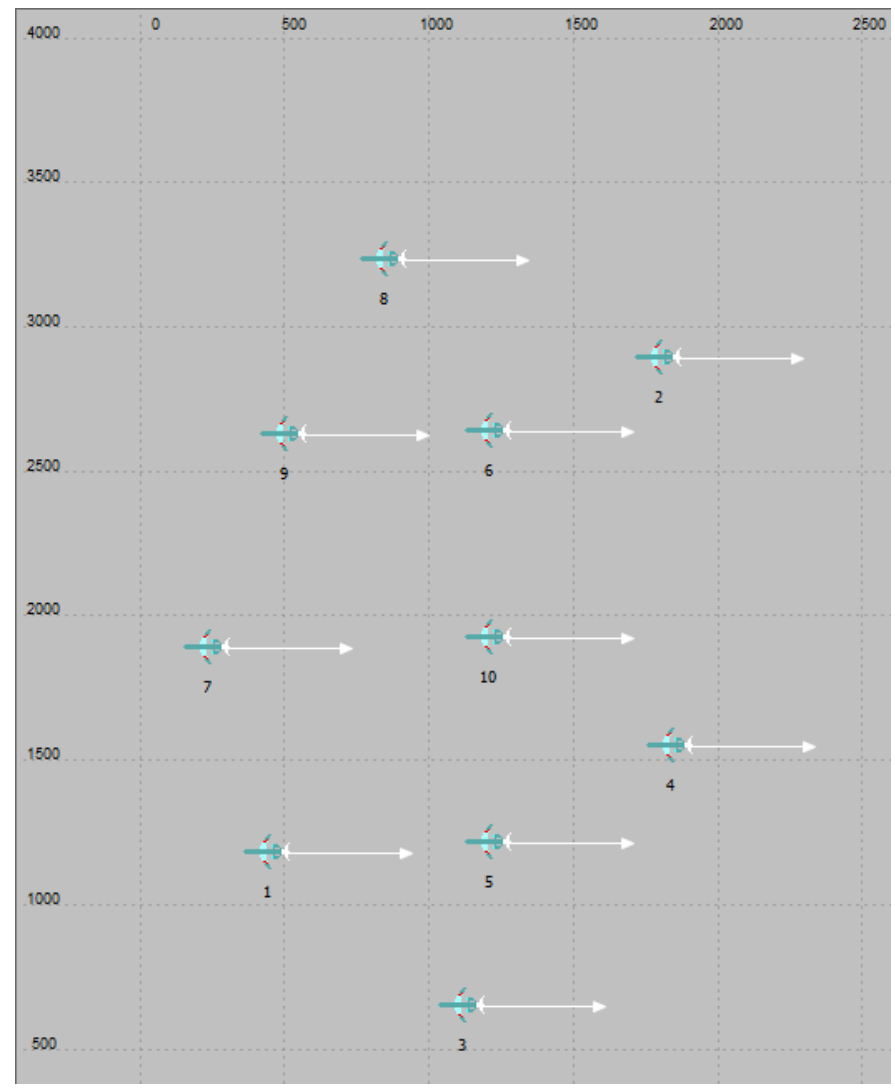

Figure B.7: Random 10-Node 5-Flow Static Scenario 7

Table B.7: Optimal Link Scheduling for 10-Node Scenario 7

\begin{tabular}{lccccc}
\hline Traffic flow & slot 1 & slot 2 & slot 3 & slot 4 & slot 5 \\
\hline \hline $8 \longrightarrow 3$ & & $(8,5)$ & $(5,3)$ & & \\
$1 \longrightarrow 2$ & $(1,2)$ & & & & \\
$5 \longrightarrow 9$ & $(5,8)$ & $(8,1)$ & $(1,9)$ & & \\
$7 \longrightarrow 4$ & $(7,3)$ & $(3,5)$ & $(5,4)$ & & \\
$3 \longrightarrow 6$ & & $(3,1)$ & $(1,6)$ & & \\
\hline
\end{tabular}




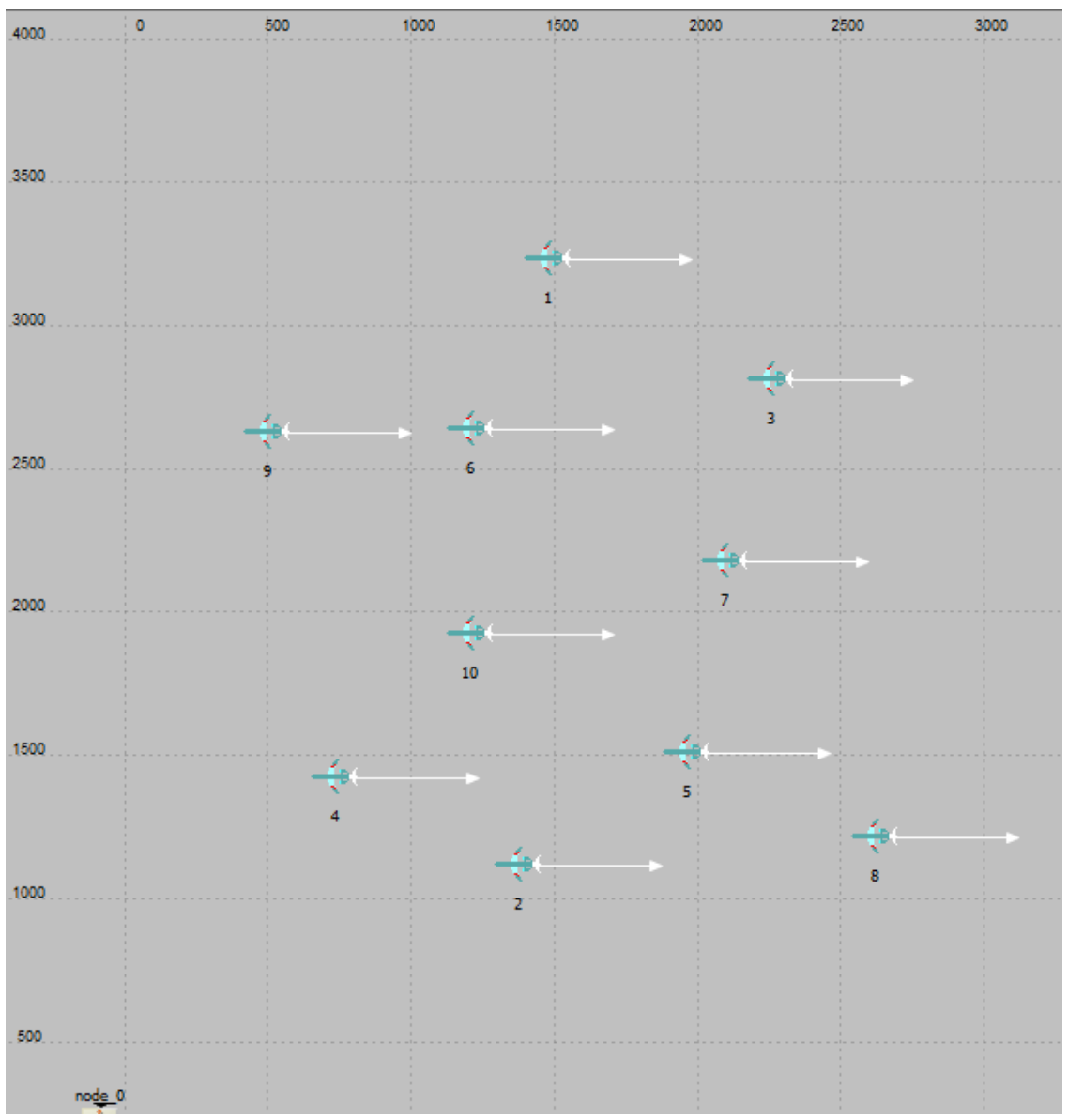

Figure B.8: Random 10-Node 5-Flow Static Scenario 8

Table B.8: Optimal Link Scheduling for 10-Node Scenario 8

\begin{tabular}{|c|c|c|c|c|}
\hline Traffic flow & slot 1 & slot 2 & slot 3 & slot 4 \\
\hline $1 \longrightarrow 2$ & & $(1,2)$ & & \\
\hline $8 \longrightarrow 9$ & $(8,1)$ & $(1,9)$ & & \\
\hline $4 \longrightarrow 3$ & $(4,5)$ & $(5,3)$ & & \\
\hline $5 \longrightarrow 6$ & & $(5,8)$ & $(8,1)$ & $(1,6)$ \\
\hline $2 \longrightarrow 9$ & $(2,6)$ & & $(6,1)$ & $(1,9)$ \\
\hline
\end{tabular}




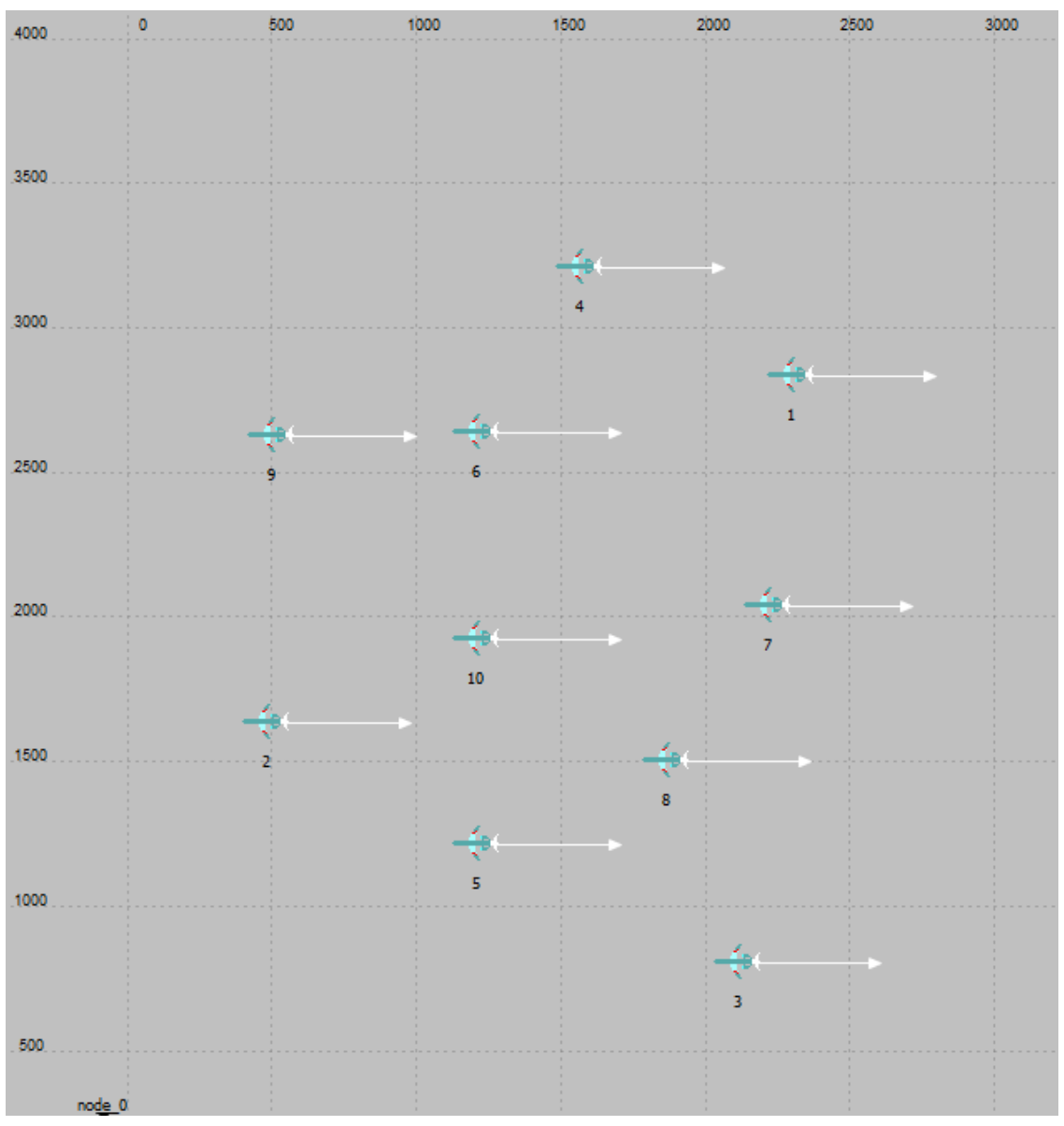

Figure B.9: Random 10-Node 5-Flow Static Scenario 9

Table B.9: Optimal Link Scheduling for 10-Node Scenario 9

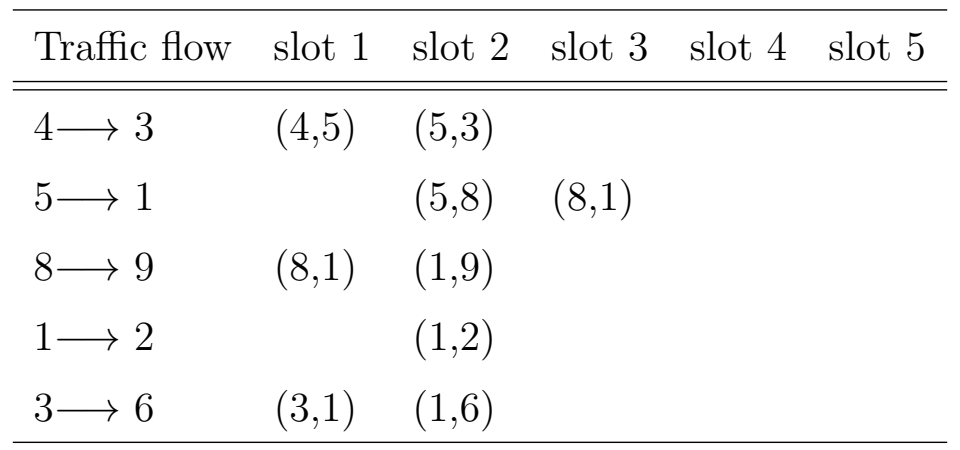




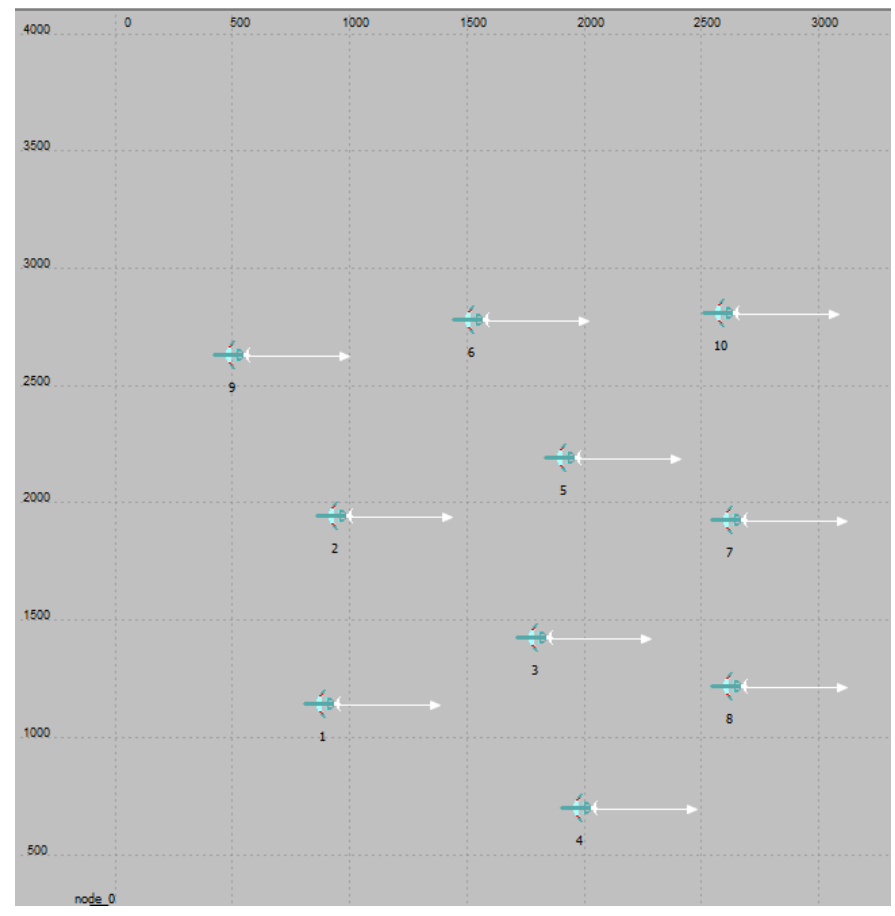

Figure B.10: Random 10-Node 5-Flow Static Scenario 10

Table B.10: Optimal Link Scheduling for 10-Node Scenario 10

\begin{tabular}{llllll}
\hline Traffic flow & slot 1 & slot 2 & slot 3 & slot 4 & slot 5 \\
\hline \hline $9 \longrightarrow 4$ & $(9,7)$ & $(7,3)$ & $(3,5)$ & $(5,4)$ & \\
$1 \longrightarrow 10$ & $(1,2)$ & $(2,8)$ & $(8,10)$ & & \\
$8 \longrightarrow 6$ & $(8,2)$ & $(2,6)$ & & & \\
$2 \longrightarrow 7$ & & $(2,1)$ & $(1,7)$ & & \\
$4 \longrightarrow 6$ & $(4,5)$ & $(5,8)$ & $(8,2)$ & $(2,6)$ & \\
\hline
\end{tabular}

